\title{
Breast reconstruction
}

Citation for published version (APA):

Kamali Sadeghian, P. (2019). Breast reconstruction: Trends and variation using big data. [Doctoral Thesis, Maastricht University]. Datawyse / Universitaire Pers Maastricht.

https://doi.org/10.26481/dis.20190417pks

Document status and date:

Published: 01/01/2019

DOI:

10.26481/dis.20190417pks

Document Version:

Publisher's PDF, also known as Version of record

\section{Please check the document version of this publication:}

- A submitted manuscript is the version of the article upon submission and before peer-review. There can be important differences between the submitted version and the official published version of record.

People interested in the research are advised to contact the author for the final version of the publication, or visit the DOI to the publisher's website.

- The final author version and the galley proof are versions of the publication after peer review.

- The final published version features the final layout of the paper including the volume, issue and page numbers.

Link to publication

\footnotetext{
General rights rights.

- You may freely distribute the URL identifying the publication in the public portal. please follow below link for the End User Agreement:

www.umlib.nl/taverne-license

Take down policy

If you believe that this document breaches copyright please contact us at:

repository@maastrichtuniversity.nl

providing details and we will investigate your claim.
}

Copyright and moral rights for the publications made accessible in the public portal are retained by the authors and/or other copyright owners and it is a condition of accessing publications that users recognise and abide by the legal requirements associated with these

- Users may download and print one copy of any publication from the public portal for the purpose of private study or research.

- You may not further distribute the material or use it for any profit-making activity or commercial gain

If the publication is distributed under the terms of Article $25 \mathrm{fa}$ of the Dutch Copyright Act, indicated by the "Taverne" license above, 


\section{BREAST RECONSTRUCTION: TRENDS AND VARIATION USING BIG DATA}

Parisa Kamali Sadeghian 



\section{BREAST RECONSTRUCTION:}

\section{TRENDS AND VARIATION USING BIG DATA}


(C) Parisa Kamali Sadeghian, Maastricht 2019

All rights reserved. No part of this publication may be reproduced or transmitted in any form or by any means without prior permission in writing from the author.

Layout \& Cover: Datawyse | Universitaire Pers Maastricht

Printing: ProefschriftMaken | www.proefschriftmaken.nl

ISBN: 978-94-6380-271-0

Printing of this thesis was financially supported by:

Netherlands Comprehensive Cancer Organisation (IKNL), Department of Plastic and Reconstructive Surgery Maastricht UMC+, Nederlandse Vereniging van Plastisch Chirurgie (NVPC), BlooMEDical Benelux NV, Emdaplast, van Wijngaarden Medical, Khalid Ouzza namens ABN AMRO group, Chipsoft 


\title{
BREAST RECONSTRUCTION:
}

\section{TRENDS AND VARIATION USING BIG DATA}

\author{
PROEFSCHRIFT \\ ter verkrijging van de graad van doctor aan de \\ Universiteit Maastricht \\ op gezag van Rector Magnificus \\ Prof. dr. Rianne M. Letchert \\ volgens het besluit van het College van Decanen \\ in het openbaar te verdedigen
}

op Woensdag 17 april 2019 om 16:00 uur

door

Parisa Kamali Sadeghian 
Supervisor

Co-supervisor

Assessment Committee
Prof. Dr. R.R.W.J. van der Hulst, MUMC+

Prof. Dr. S.J. Lin, Harvard Medical School, Boston

Dr. H.A. Rakhorst, Medisch Spectrum Twente

Prof. Dr. V.C.G. Tjan-Heijnen, chair, MUMC+

Prof. Dr. A. Dekker, MUMC+

Dr. E. Heuts, MUMC+

Prof. Dr. V. Lemmens, Erasmus MC Rotterdam,

Dr. M. Vrancken-Peeters, Netherlands Comprehensive Cancer

Organisation (IKNL), Amsterdam 

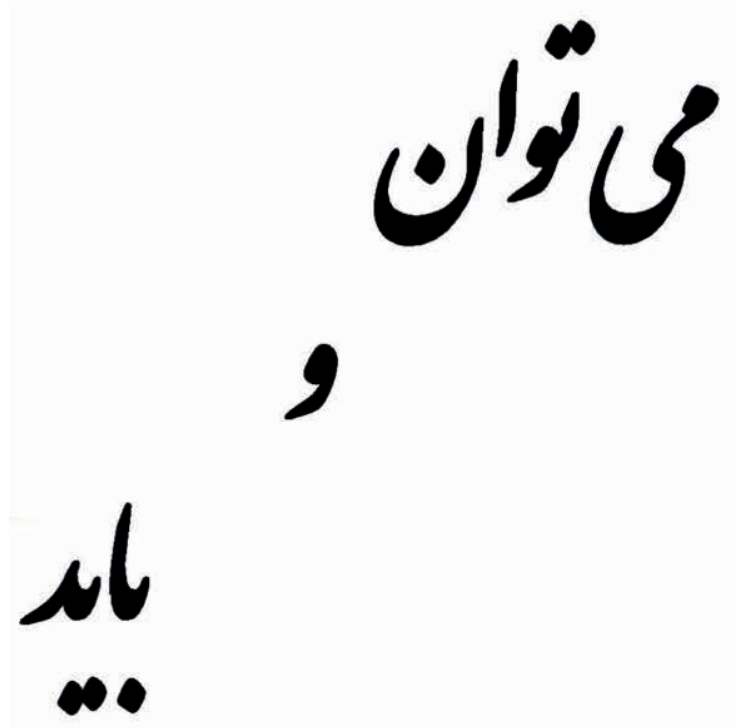

9

We can and therefore we must

For my parents 



\section{Table of Contents}

Chapter 1

General Introduction and Outline of Thesis

Chapter 2

25

Analyzing Regional Differences over a Fifteen-year Trend of One-stage versus Two-staged Breast Reconstruction in 941,191 Post-Mastectomy Patients

\section{Chapter 3}

National and Regional Differences in 32,248 Post-Mastectomy Donor Sites for Breast Reconstruction Patients Using the Updated National Inpatient Survey

\section{Chapter 4}

Immediate Breast Reconstruction among Patients with Medicare and Private Insurance: A Matched Cohort Analysis

\section{Chapter 5}

Trends In Immediate Breast Reconstruction and Early Complication

Rates Among Older Women: A Big Data Analysis

\section{Chapter 6}

The National Surgical Quality Improvement Program 30-Day Challenge:

Microsurgical Breast Reconstruction Outcomes Reporting Reliability

\section{Chapter 7}

Prevalence of Psychiatric Comorbidities Among Women Undergoing

Free Tissue Autologous Breast Reconstruction

\section{Chapter 8}

Differences in the Reporting of Racial and Socioeconomic Disparities among Three Large National Databases for Breast Reconstruction

\section{Chapter 9}

Immediate Breast reconstruction in the Netherlands and the United States: a Proof of Concept to Internationally Compare Quality of Care Using Cancer Registry Data 
Chapter 10

Discussion and future perspectives

Chapter 11

Summary

Nederlandse Samenvatting/Dutch Summary

\section{Appendices}

Valorization

Acknowledgement

Curriculum Vitae

List of publications 


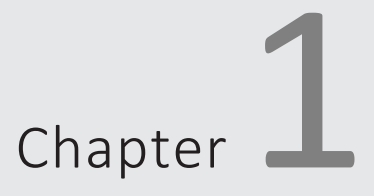

General Introduction and Outline of Thesis 
In the ever-evolving realm of clinical outcomes analysis and with the ease at which information can be digitalized, "big data" has quickly become one of the most important buzzwords of the last decade. Although it has been making waves in other industries for quite some time, its application in healthcare is still in the formative stages. ${ }^{1-3}$ Healthcare organizations have traditionally generated large amounts of data in the form of claims and cost data, pharmaceutical data, research and development data, clinical (outcomes) data, and patient behavior or sentiment data. ${ }^{4}$ While most data are stored in hard copy form, since the advent of health information technology (HIT), these data have emerged as a "natural resource" that could potentially revolutionize how we deliver personalized medicine and improve healthcare outcomes. ${ }^{2,4}$ Moreover, the implementation of the electronic health records (EHR) has resulted in a large volume of individual health data. With the creation of massive datasets, these data hold great potential to change the whole healthcare value chain. Some argue that we are on the edge of the next revolution in medicine. Research using big data has had a significant impact on surgical outcomes research including breast reconstruction. This has resulted in changes to treatment guidelines and health policy. ${ }^{5-}$ ${ }^{13}$ That being said, the concept, potential use and power of big data within the field of breast reconstruction is still underutilized. The current work aims to use big data in the form of large-volume national databases to evaluate the current standard of care in reconstructive breast surgery.

\section{WHAT IS BIG DATA?}

Big data is not a new concept or idea. The abstract idea of "big data" was first encountered over 70 years ago, but not until recent decades have industries begun to actively take advantage of what has been termed the "information explosion". ${ }^{14}$ The first documented use of the term "big data" appeared in a 1997 paper by scientists at NASA, describing the problem they had with visualization (i.e. computer graphics) which "provides an interesting challenge for computer systems: data sets are generally quite large, taxing the capacities of main memory, local disk, and even remote disk" ${ }^{15}$ In 2008, a number of prominent computer scientists popularized the term, predicting that "big-data computing" would "transform the activities of companies, scientific researchers, medical practitioners, and the nation's defense and intelligence operations" ${ }^{16}$ The definition of big data has been debated for over a decade, and its use is continuously being reconsidered. ${ }^{17}$ To date, a universally accepted definition of big data does not exist. ${ }^{18}$ Generally speaking, big data are frequently characterized as the four Vs: volume, velocity, variety, and veracity. ${ }^{3,17,19}$ More recently, an additional two Vs have been adopted for the healthcare sector: variability and value. ${ }^{17,20}$ 
Volume refers to the volume of data per "transaction". This has gone from bytes (e.g. traditionally recording observations every 6 hours), to kilobytes (e.g. clinic letters in Electronic Health Records), to megabytes (e.g. clinical photographs), to gigabytes (e.g. $\mathrm{CT} \& \mathrm{MRI}$ scans, genomic sequencing). Every day, we create 2.5 trillion gigabytes of data. This is increasing at such as rapid rate that $90 \%$ of all data worldwide was created in only the last two years. ${ }^{21}$ Furthermore, the volume of stored information in the world is growing so fast that scientists have had to create orders of magnitude of data, including zettabyte ( $10^{21}$ gigabytes) and yottabyte ( $10^{24}$ gigabytes) to describe the flood of data. ${ }^{4,17,22}$ In 2012, the magnitude of worldwide healthcare data was 500 petabytes ( $10^{6}$ gigabytes), and it is estimated that this number will grow by $50 \%$ to 25,000 petabytes in 2020. ${ }^{4}$ That being said, recent studies have estimated that less than 3 percent of potentially useful big data underwent any type of analysis. ${ }^{23}$ As genomic and environmental data become more ubiquitous, coupled with greater use of precision medicine, there will be an explosion of big data in healthcare.

Velocity refers to the speed at which processes occur to make data available for use. Big data offers the ability for data to be processed in real-time. Traditionally, health care data has been mostly static, however real-time accumulation of data has significant advantages, particularly in the monitoring of vital signs and laboratory results. For example, as more and more medical devices are designed to monitor patients and collect data, there is great demand for analysis of that data and then to transmit it back to clinicians and others.

With increasing volume and velocity comes increasing variety. Traditionally, it has been structured such that data has been recorded electronically. Structured data (patient names, diagnostic codes, traditional databases etc.) is standardized, pre-defined data that can easily be stored, manipulated and analysed. However, over $80 \%$ of data collected in the healthcare setting is in an unstructured or "raw" format, collected in what is known as a Data Lake, and on paper.

Veracity refers to the quality, relevance, predictive value, and meaning of data. Veracity is important for big data as, for example, personal health records may contain typographical errors, abbreviations and cryptic notes. Ambulatory measurements are sometimes taken within less reliable, uncontrolled environments compared to clinical data, which are collected by trained practitioners.

Variability of data can be translated to the way care is provided to any given patient and is dependent on different factors-and the way the care is delivered. Perhaps more importantly the way the data is captured may vary from time to time or place to place. For example, what a clinician reads in the medical literature, where they trained, the professional opinion of a colleague down the hall, or how a patient expresses herself during her initial exam all may play a role in what happens next. Such variability means data can only be meaningfully interpreted when care setting and delivery process is 
taken into context. For example, a diagnosis of " $\mathrm{CP}$ " may mean chest pain when entered by a cardiologist or primary care physician but may mean "cerebral palsy" when entered by a neurologist or paediatrician. Because true interoperability is still somewhat elusive in health care data, variability remains a constant challenge.

And finally, the value of data relates to the clinical relevancy of data or in other words, the worth of information to various stakeholders or decision makers. Many of the underlying principles of big data have been explored by the research community for years in other domains. Nevertheless, new theories and approaches are needed for analysing big health data.

\section{BIG DATA IN HEALTHCARE AND PLASTIC SURGERY}

Medicine has traditionally been a science of observation and experience. For thousands of years, clinicians have integrated the knowledge of preceding generations with their own life-long experiences to treat patients according to the Hippocratic Oath; mostly based on trial and error. Over the years, the generation of information has changed dramatically. Driven by record keeping, compliance and regulatory requirements, and patient care, the healthcare industry had historically produced a large amount of data. ${ }^{4}$ These rich data sources offer many potential benefits. The evolution in HIT infrastructure over recent decades, and the corresponding ability to store, share, manipulate and process large amounts of data have resulted in a significant increase in big data outcomes research. Big data on its own is just the dataset; it is the analytics of such data that will have the greatest impact on research and clinical practice. As technology has developed for the storage of such data, so has the ability to analyze it.

To date, plastic surgeons have traditionally used their judgement when making treatment decisions, but in the last few years there has been a shift towards evidencebased medicine, which involves systematically reviewing clinical data and making treatment decisions based on the best available information. ${ }^{24-26}$ The scale and comprehensive nature of large data in healthcare allow for analyses that would otherwise be impossible with single surgeon or health-system database reviews. With the magnitude and breadth of information available in these databases, researchers are able to more easily answer a wide variety of research questions.

Within the field of plastic and reconstructive surgery, research using big data has increased dramatically over the past decade. ${ }^{5-13}$ Although prospective, randomized controlled trials (RCTs) are considered the gold standard for developing clinical care guidelines, randomization is not always practical or ethical, and the results of such studies may not be applicable for all plastic surgeons and their patients. ${ }^{27-30}$ An adequately powered trial requires a large number of patients, often from multiple centres, with the logistics of this making RCTs very costly. Furthermore, although 
blinding to placebo or "sham surgery" treatments are important to limit bias, it may be unethical in many cases. A well-designed observational study, carefully controlling for potential sources of bias, may provide a strong level of evidence, which approaches that of an RCT. Moreover, database methodologies may be beneficial as they offer ease in recruitment, decreased cost, reduction in some types of bias, and greater generalizability as the data in these patient registries are readily available and contains a multitude of patients from different populations.

\section{LARGE-VOLUME DATABASES}

Large-volume national databases can include all patients or a predefined sample of patients from a broad (national) or narrow (state) geographic area, and are composed of patient-encounter records from more than one specialty or procedure. They offer a unique platform for observational and clinical studies providing large patient populations suitable for reviewing outcomes that would otherwise be difficult to study by a single plastic surgeon, institution, or even group of institutions. As data collection is done preemptively, these studies can be completed at low cost, and with careful study design. Thus, the inherent biases of retrospective studies can be controlled for. The lack of plastic surgical- specific data elements in many of the current general surgeryfocused national databases does limit the ability to assess plastic surgery outcomes of interest such as measures of function in hand surgery, and the ability to control for factors that influence outcomes, for example, the type of implant or type of flap used for breast reconstruction. Nevertheless, the wealth of data available in these national datasets has already allowed several impactful studies to be completed. Large-volume databases provide a unique opportunity for observational plastic surgical research to answer questions that cannot be examined in most RCTs. Observational database research typically provides nationwide information from a variety of patients, providers and settings. In contrast to RCTs, which often have relatively small samples, strict patient selection criteria, and select providers, database studies provide real-world healthcare information from a broader, population-based perspective.

\section{Administrative or clinical databases}

Generally speaking, large-volume national databases can be broadly categorized as either administrative or clinical. This is mostly dependent on where the data is derived from. Administrative database contains diagnosis and procedure codes used primarily for billing purposes and have a broader, less selective catchment. These databases were not originally designed for clinical research, but rather to track billing for hospitals, providers, and procedures. Administrative data are derived from two sources: requests to an insurer for health care payments and claims for clinical services and therapies. 
Utilizing international classification of disease clinical medicine (ICD-9-CM) codes for diagnoses and current procedural terminology (CPT) codes, administrative databases provide a mechanism to look at population-based data. The large number of patients allows for sufficient powering of research studies. Topics such as operative trends may be assessed using longitudinal databases spanning several years. Additional outcomes including length of stay and hospital cost may be assessed with large administrative databases. Only limited patient characteristics (e.g. age, sex, postcode and presentation) along with diagnosis and procedure information are typically recorded.

Unlike administrative databases, clinical databases were developed with specific clinical goals in mind. Eligibility may be disease-based or procedure/device-based. In contrast to administrative data, important relevant clinical factors are collected, as are a wider range of outcomes and information on co-morbidities. Cancer registries track incidence, treatment, and differences in cancer care. Disease-specific information may include stage, tumor characteristics, treatment modalities, and outcome. Quality improvement databases aim to assess the quality of patient care. Preselected quality measures including intraoperative measures, 30-day morbidity and mortality are reported.

These large-volume databases have several unique advantages that have fuelled their popularity amongst plastic surgeon-researchers. Derivation of data from large, heterogeneous populations may result in decreased selection bias and increased study generalizability. ${ }^{31,32}$ New surgical interventions and assessment of quality improvement measures may be examined. ${ }^{33}$ Moreover, assessment of cost and effectiveness of plastic surgical treatments, variation at the regional, state and national level can be assessed, and risk models can be created and validated.

\section{LARGE-VOLUME DATABASES USED IN THIS THESIS}

\section{National Inpatient Sample (NIS) database}

The Healthcare Cost and Utilization Project (HCUP) is a federally mandated program managed by the Agency of Healthcare Research and Quality. The HCUP is composed of several regional and national databases, the largest of which is the Nationwide Inpatient Sample (NIS) database. Across all publicly available databases, the NIS is the largest allpayed inpatient (administrative) database in the United States. The database aggregates information for over 1,000 hospitals, over 8 million discharges, and dates back to 1988 . The database does not record each individual discharge from a given institution but rather records a $20 \%$ stratified systemic random sample of all discharges. Each discharge can contain over 100 data points allowing for the stratification of variables by region, ownership (i.e. public versus private), location (i.e. urban versus rural), size, and resident educational programs. The data provided by NIS is unweighted, therefore 
when conducting studies investigating nationwide trends, it is important to use appropriate weighting variables that are provided by $\mathrm{HClP}$ and determined by an institution's relative contribution to the NIS. Stratification of patient or procedural information is achieved through International Classification of Diseases $9^{\text {th }}$ edition (ICD9) procedural and diagnostic codes.

Significant strengths of the NIS database are found in its overall number of patients and the volume of data recorded with each discharge. Additionally, access to the database is relatively affordable at $\$ 160-\$ 350$ per year of data requested. Currently, it is the largest national database composed of all payers, and is frequently used for nationwide analysis of a given procedure.

There are several limitations of the NIS database that are important to consider in any study utilizing this database. First, the NIS database only records inpatient events and does not allow for longitudinal tracking of patients. Therefore, only inpatient complications may be assessed for a given procedure, which likely underestimates the true complication rate. A second major limitation is the database only contains predischarge information, making it difficult to distinguish comorbidities from complications. Lastly, it does not contain operative variables such as anaesthesia type, length of surgery, or blood loss which can be important variables to consider in surgical research. Despite the limitations of the NIS database, it remains commonly utilized in plastic surgical research.

\section{American College of Surgeons National Surgical Quality Improvement Program (ACS-NSQIP)}

The ACS-NSQIP database has its origins in the Veterans Affairs (VA) healthcare system. In the 1980s, the United States government developed the Veterans Affairs Surgical Quality Improvement Program (VASQIP) after noticing high rates of morbidity and mortality following surgical procedures in VA patients. After the noted success of the VASQIP in decreasing perioperative complications, its methods were implemented in private sector hospitals and found to be similarly effective. The current NSQIP database was developed by the American College of Surgeons to improve the quality of surgical care. It is a nationally validated database, and studies have demonstrated the ability of the NSQIP to improve surgical outcomes.

The NSQIP database is peer-controlled and collects information on patients who undergo elective and select trauma surgical procedures. Contributing institutions are located both within the United States and abroad. The database records a wide array of clinical information in addition to surgery-specific variables such as operative time, blood transfusion, anaesthesia type, and estimated blood loss. The data recorded in the NSQIP database dates back to 2006. A major strength of the database is that data is abstracted by thoroughly trained "nurse reviewers" who use a standardized set of definitions, and inclusion and exclusion criteria. These reviewers may track greater than 
135 data points for a given patient undergoing a surgical procedure. The inter-rater reliability of data extraction by these reviewers proves to be consistent.

The primary limitation of the NSQIP database is it only tracks 30-day morbidity and mortality after a surgical procedure. The database also does not provide specific information regarding severity of comorbidities, indications for surgery, or specific outcomes related to a given procedure. Lastly, free access to the database is limited to contributing institutions, and the infrastructure to contribute to the NSQIP may exceed $\$ 100,000$ as a full- time "nurse reviewer" is needed. This high cost has led to a disproportionate contribution to the NSQIP from large teaching hospitals. Aside from its limitations, the NSQIP database is widely believed to be the most precise and accurate database available for measuring patient outcomes after surgery given its high rate of complete data sets, operative data points, validation, and inter-rater reliability

\section{Surveillance, Epidemiology, and End Results (SEER) program}

The Surveillance, Epidemiology, and End Results (SEER) program of the National Cancer Institute $(\mathrm{NCl})$ is a source of epidemiologic information on the incidence and survival rates of cancer in the United States. The SEER program was launched in 1973 as part of the National Cancer Act. SEER collects demographic, clinical and outcome information on all cancer diagnosed representative geographic regions and subpopulations. Regions are included based on their ability to operate and maintain a high-quality populationbased cancer reporting system or Cancer Registry and to enhance the demographic and geographic diversity of the SEER data. The population covered by SEER is representative of the general U.S. population in regard to measures of poverty and education. SEER currently captures 400,000 cancer cases annually and stores cancer data on approximately $30 \%$ of the U.S. population.

The pathology report is an important source for abstracting SEER data, and for approximately $80 \%$ of cases, pathology reports are obtained electronically in real-time from approximately 360 laboratories. The abstracted pathology data are consolidated into a final case record along with data from other sources. These sources include patient medical records, reports gathered from freestanding diagnostic imaging and chemotherapy clinics, and death certificates. Traditionally cancer registry staff members abstract standard data items, and manually enter corresponding text into a data collection template. The use of electronic pathology reporting by nearly $80 \%$ of laboratories has the potential to enable the use of natural language processing (NLP) software to automatically code data fields. These abstracted records are then reviewed by the registry staff. Afterwards, all data are checked, edited and incorporated into the annual registry database, and submitted in a de-identified form to the $\mathrm{NCl}$. SEER submissions are checked for quality and completeness and released for public use and access in the subsequent year. 
According to the SEER website, the best use of SEER data leverages the programs strengths with regard to representativeness and generalizability to the U.S. population, lengthy period of data collection, large numbers of cases, and collection of cancer specific outcomes. Limitations include incompleteness of individual-level data collected on specific cancer risk and treatment, and inaccuracies and incompleteness of the data collected from the source registries. Further, data related to socioeconomic status, comorbidities and other health information are lacking. Tumor recurrence data are currently not collected and therefore progression free survival, correlates of local, regional and distant control, and the effectiveness of salvage therapy cannot be assessed. Moreover, survival outcome cannot be fully evaluated as the collected data does not distinguish the intent of therapy as curative or palliative. Specific details on the type, dose and duration of chemo- and radiation therapy, and the use of other oral pharmaceuticals are not collected. Information gaps in treatment and follow up occur when individuals migrate into and out of SEER and non-SEER regions and could bias conclusions about a cancer behaviour, particularly if re-location and outcome are linked. Such interruptions in data collection also occur when individuals within a SEER region have a procedure in a facility which has no contractual obligation to transmit data to SEER or have prescriptions in pharmacies or diagnostic/predictive molecular tests in commercial laboratories who are not obligated to transmit their data to SEER. Inaccuracies in the source data can occur either due to miscoding of the data transmitted to $\mathrm{NCl}$ by the regional registries or the data made available to the regional registrars for coding is not correct. The SEER quality program is designed to identify miscoding of the transmitted data and acts quickly to rectify any identified error.

\section{National Breast Cancer Organization the Netherlands Breast Cancer Audit (NBCA)}

The NBCA is a national multidisciplinary quality improvement audit developed in 2011 as part of the Nationaal Borstkanker Overleg Nederland (NABON). The registry contains data on all patients diagnosed with invasive breast cancer (BC) or ductal carcinoma in situ (DCIS) from all hospitals $(n=92)$ in the Netherlands. Hospitals can choose to collect data themselves or have data-managers of the Netherlands Cancer Registry (NCR) register the data (the latter option being used by $75 \%$ of hospitals). By providing feedback to the participating individual hospitals on their outcomes, the NBCA enables institutions to evaluate their data and start improvement projects. ${ }^{34,35}$ In 2015, the database included 32 quality indicators measuring the structure, processes, and outcomes of breast cancer care. ${ }^{34-36}$ For research, an official request must be made to the scientific committee of the NBCA and the NCR, who judge the relevance of the research question and privacy of the patients, care givers and institutions. 


\section{BREAST RECONSTRUCTION AND BIG DATA}

Breast cancer is the most commonly diagnosed cancer among women worldwide. With nearly 316,120 new cases diagnosed in 2017 in the US alone, resulting in 40,610 deaths, breast cancer accounts for $25 \%$ of all new cancer cases in women. Widely accepted statistics in breast cancer are based on publication called GLOBOCAN, a project of the International Agency for Research on Cancer (IARC). Timely and accurate breast cancer statistics are crucial in identifying priorities for cancer control strategies at the national level. Yet, only 34 of 194 World Health Organization (WHO) Member States presently report high-quality national mortality data, while 68 countries provide high-quality incidence data. As a result, many policy-makers rely on national cancer incidence and mortality estimates of variable precision to inform cancer control priorities. GLOBOCAN provides estimates by cancer site and sex using the best available data in each country and several methods of estimation. As seen in this global and widely accepted project, the importance of big data and reliable patient registries are of utmost importance.

Several treatment options are available for patients with breast cancer or ductal carcinoma in situ (DCIS), such as surgery, chemotherapy, radiation therapy and hormonal therapy. Despite the introduction of breast conserving therapy with or without neo-adjuvant treatment, it is estimated that 40 to 55 percent of women diagnosed with breast cancer or DCIS opt for mastectomy. And yet again, the international community relies on data provided by national large-volume registries.

\section{Mastectomy and Breast Reconstruction}

Mastectomy has been shown to have a significant impact on a woman's body image and self-identity, including feelings of abnormality, depressive symptoms, loss of wholeness, and mourning for the loss of a breast. ${ }^{37}$ Women who undergo a mastectomy as part of their breast cancer treatment may choose breast reconstruction surgery to rebuild the shape and look of their breast. Breast reconstruction following mastectomy has been shown to improve quality of life, body image, and sexual well-being. It can be performed immediately following mastectomy (immediate breast reconstruction; IBR), or delayed for months or years after mastectomy (delayed breast reconstruction; DBR). ${ }^{37,38}$ There are a variety of different reconstruction techniques available. The two main techniques include

- Implant based breast reconstruction: Inserting an implant that is filled with saline, silicone gel, or a combination of the two.

- Autologous or "flap" reconstruction: Using tissue transplanted from another part of the body. Autologous reconstruction also may include an implant.

Research derived from big data captured in large-volume databases have made a significant impact in the field of breast reconstructive surgery. For example, in the 
United States The Women's Health and Cancer Rights Act (WHCRA) was federally enacted in 1998 to ensure that all group and individual health plans provide insurance coverage for reconstruction after mastectomy regardless of diagnosis. This legislation represented a paradigm shift in the availability of reconstruction by eliminating economic hurdles to care. Although a greater number of women have opted for breast reconstruction in the years after the WHCRA, this trend was not consistent across all demographic groups. ${ }^{8,39-41}$ Studies based on large-volume databases revealed significant disparities based on access to health care, age, race, geographic location, and patient education. As a result, patient education events, such as Breast Reconstruction Awareness Day instituted in 2012, have emerged. In 2015, the Breast Cancer Patient Education Act was designed to implement an educational campaign to inform women in minority and lower socioeconomic groups about their options for breast reconstruction. This bill referred to statistics provided by the American Cancer Society, multiple institutional studies, and in terms of large-volume database, studies based on SEER data.

Using big data, this thesis aimed to evaluate a variety of different aspects within the field of breast reconstructive surgery:

1. Can we evaluate the trend over time and examine disparities in different types of breast reconstructive techniques? (chapter 1 and 2)

2. Using big data; is it possible to evaluate and measure the effects of laws and legislations in relation to breast reconstructive surgery? (chapter 3)

3. How can we bring big data back to clinical relevance in daily practice? (chapter 4)

4. Is administrative and clinical "big data" comparable to (traditional) institutional data? (chapters 5 and 6)

5. How comparable are big data large-volume databases, both nationally and internationally? (chapters 7 and 8 ) 


\section{OUTLINE THESIS}

\section{Evaluating Trends}

Using big data collected from the largest administrative database in the United States, the Nationwide Inpatient Database (NIS), in chapter 1, we evaluated the national and regional trends over 15 years for immediate (one-stage) or delayed (two-stage) implantbased breast reconstruction. We extended the use of the NIS in chapter 2 by evaluating the national and regional trends in the United States over 5 years to compare different types of autologous breast reconstruction in postmastectomy patients.

\section{Laws and Legislation}

By eliminating economic hurdles, the Women's Health and Cancer Rights Act of 1998 represented a paradigm shift in the availability of breast reconstruction. Yet, studies report disparities among Medicare-insured women. These studies do not account for the inherent differences in age and comorbidities between a younger privately insured individual and an older Medicare population. In chapter 3, we examine immediate breast reconstruction utilization between a matched pre- and post-Medicare population using the NIS database.

\section{Disparities}

Although approximately $57 \%$ of breast cancer diagnoses are in older patients ( $>60$ years), only $4.1-14 \%$ undergo breast reconstruction. This has been attributed to physician concerns about operative complications. In chapter 4 we use a clinical database, the NSQIP, to analyze the 30-day complication rates in an elderly patient population undergoing immediate breast reconstruction. Moreover, we use this database to analyze links between complication type and category of reconstruction.

\section{Big Data and Traditional Outcomes Research}

In chapter 5 and 6 we revert back to traditional outcomes research using big data. Chapter 5 compares the 30-day complication rates in immediate breast reconstruction within the NSQIP database to our institutional 30-day complication data and assesses those that occur after 30 days. For immediate autologous tissue breast reconstruction, the NSQIP may be reliable for monitoring and comparing surgical site infection, wound disruption, unplanned reoperation, and unplanned readmission rates. However, clinicians may find it useful to understand the limitations of the ACS-NSQIP for complications and risk factors, as it may underreport complications occurring beyond 30 
days. Chapter 6 compared the rates of psychiatric disorders in patients undergoing free autologous breast reconstruction captured in the NIS and our institutional database.

\section{Comparing National and International Datasets}

Although big data has been used in different forms to evaluate trends of immediate reconstruction after breast cancer, to date, a comparison of the different databases has not been done. Research derived from large-volume databases plays an increasing role in the development of clinical guidelines and health policy. In breast cancer research, the SEER, NSQIP, and NIS databases are widely used. Chapter 7 aims to compare the trends in immediate breast reconstruction and identify the drawbacks and benefits of each database.

There are many clinical and administrative databases around the globe. Still, the ability to compare data within them is greatly limited by the heterogeneity of the data collected, their definitions, and coding. To date, no comparison between large databases has been made internationally within the field of breast cancer outcomes. Chapter 8 is the first proof of concept study to explore the feasibility of combining two large existing operational databases in two countries; e.g. SEER in the United States and NBCA in the Netherlands. 


\section{REFERENCES:}

1. Ryu S, Song TM. Big data analysis in healthcare. Healthcare informatics research. 2014;20(4):247-248.

2. Mathias B, Lipori G, Moldawer LL, Efron PA. Integrating "big data" into surgical practice. Surgery. 2016;159(2):371-374

3. Lee $\mathrm{CH}$, Yoon $\mathrm{HJ}$. Medical big data: promise and challenges. Kidney research and clinical practice. 2017;36(1):3-11.

4. Raghupathi W, Raghupathi V. Big data analytics in healthcare: promise and potential. Health information science and systems. 2014;2:3.

5. Kamali P, Curiel D, van Veldhuisen $C L$, et al. Trends in immediate breast reconstruction and early complication rates among older women: A big data analysis. Journal of surgical oncology. 2017;115(7):870-877

6. Kamali P, Koolen PG, Ibrahim AM, et al. Analyzing Regional Differences over a 15-Year Trend of OneStage versus Two-Stage Breast Reconstruction in 941,191 Postmastectomy Patients. Plastic and reconstructive surgery. 2016;138(1):1e-14e.

7. Kamali P, Paul MA, Ibrahim AM, et al. National and Regional Differences in 32,248 Postmastectomy Autologous Breast Reconstruction Using the Updated National Inpatient Survey. Annals of plastic surgery. 2017.

8. Kamali P, Zettervall SL, Wu W, et al. Differences in the Reporting of Racial and Socioeconomic Disparities among Three Large National Databases for Breast Reconstruction. Plastic and reconstructive surgery. 2017;139(4):795-807.

9. Wexelman B, Schwartz JA, Lee D, Estabrook A, Ma AM. Socioeconomic and geographic differences in immediate reconstruction after mastectomy in the United States. The breast journal. 2014;20(4):339346.

10. Chen AD, Kamali P, Chattha AS, et al. The National Surgical Quality Improvement Program 30-Day Challenge: Microsurgical Breast Reconstruction Outcomes Reporting Reliability. Plastic and reconstructive surgery Global open. 2018;6(3):e1643.

11. Bucknor A, Chattha A, Wu W, et al. The Impact of Surgical Volume on Outcomes and Cost in Cleft Repair: A Kids' Inpatient Database Analysis. Annals of plastic surgery. 2018;80(4 Suppl 4):S174-S177.

12. Epstein S, Tran BN, Cohen JB, Lin SJ, Singhal D, Lee BT. Racial disparities in postmastectomy breast reconstruction: National trends in utilization from 2005 to 2014. Cancer. 2018.

13. Lazzati A, Katsahian S, Maladry D, Gerard E, Gaucher S. Plastic surgery in bariatric patients: a nationwide study of 17,000 patients on the national administrative database. Surgery for obesity and related diseases : official journal of the American Society for Bariatric Surgery. 2018.

14. "A Very Short History of Big Data", by Gil Press, Forbes, May 9, 2013. Available at: http://www.forbes.com/ sites/gilpress/2013/05/09/a-very-short-history-of-big-data/print/. Accessed 4/5/2018.

15. Cox M, Ellsworth D. Application-controlled demand paging for out-of-core visualization. Proceedings of the 8th conference on Visualization '97; 1997; Phoenix, Arizona, USA.

16. Bryant R. E., Katz R. H., \& Lazowska E. D. (2008). Big-Data Computing: Creating revolutionary breakthroughs in commerce, science, and society: A white paper prepared for the Computing Community Consortium committee of the Computing Research Association. http://cra.org/ccc/resources/ccc-ledwhitepapers/.

17. Glick M. Taking a byte out of big data. Journal of the American Dental Association. 2015;146(11):793794.

18. Baro E, Degoul S, Beuscart R, Chazard E. Toward a Literature-Driven Definition of Big Data in Healthcare. BioMed research international. 2015;2015:639021.

19. Bellazzi R. Big data and biomedical informatics: a challenging opportunity. Yearbook of medical informatics. 2014;9:8-13.

20. Andreu-Perez J, Poon CC, Merrifield RD, Wong ST, Yang GZ. Big data for health. IEEE journal of biomedical and health informatics. 2015;19(4):1193-1208. 
21. Big Data in Healthcare: Definition, application, and challenges. Nictiz (expertise centre for e-health) 2017 Report. Available at: https://www.nictiz.nl/wp-content/uploads/2018/04/Big_Data_in_healthcare.pdf Assessed 4/5/2018

22. IHTT . Transforming Health Care through Big Data Strategies for leveraging big data in the health care industry. 2013.

23. John Gantz DR. The Digital Universe in 2020: Big Data, Bigger Digital Shadows, and Biggest Growth in the Far East. IDC. 2012;December 2012:11.

24. Goss CH, Tefft N. Comparative effectiveness research - what is it and how does one do it? Paediatric respiratory reviews. 2013;14(3):152-156.

25. Cheung MC, Allan BJ, Yang R, Thaller SR. Evidence-based medicine and its role in plastic surgery. The Journal of craniofacial surgery. 2011;22(2):385-387.

26. Rohrich RJ. So you want to be better: the role of evidence-based medicine in plastic surgery. Plastic and reconstructive surgery. 2010;126(4):1395-1398.

27. Adkinson JM, Casale MT, Kim JY, Khavanin N, Gutowski KA, Gosain AK. So You Have a Research Idea: A Survey of Databases Available for Plastic Surgery Research. Plastic and reconstructive surgery. 2016;137(2):680-689.

28. Royall RM, Bartlett RH, Cornell RG, et al. Ethics and statistics in randomized clinical trials. Statistical science : a review journal of the Institute of Mathematical Statistics. 1991;6(1):52-88.

29. Chung KC, Swanson JA, Schmitz D, Sullivan D, Rohrich RJ. Introducing evidence-based medicine to plastic and reconstructive surgery. Plastic and reconstructive surgery. 2009;123(4):1385-1389.

30. Malay S, Shauver MJ, Chung KC. Applicability of large databases in outcomes research. The Journal of hand surgery. 2012;37(7):1437-1446.

31. Deyo RA, Taylor VM, Diehr P, et al. Analysis of automated administrative and survey databases to study patterns and outcomes of care. Spine. 1994;19(18 Suppl):2083S-2091S.

32. Abdullah F, Ortega G, Islam S, et al. Outcomes research in pediatric surgery. Part 1: overview and resources. Journal of pediatric surgery. 2011;46(1):221-225.

33. Begg CB, Cramer LD, Hoskins WJ, Brennan MF. Impact of hospital volume on operative mortality for major cancer surgery. Jama. 1998;280(20):1747-1751.

34. NABON Breast Cancer Audit (NBCA). Available at: https://www.dica.nl/nbca/home. Assessed: June 1st 2016.

35. van Bommel AC, Spronk PE, Vrancken Peeters MT, et al. Clinical auditing as an instrument for quality improvement in breast cancer care in the Netherlands: The national NABON Breast Cancer Audit. Journal of surgical oncology. 2016.

36. van Bommel AC, Spronk PE, Vrancken Peeters MT, et al. Clinical auditing as an instrument for quality improvement in breast cancer care in the Netherlands: The national NABON Breast Cancer Audit. Journal of surgical oncology. 2017;115(3):243-249.

37. Dean C, Chetty U, Forrest AP. Effects of immediate breast reconstruction on psychosocial morbidity after mastectomy. Lancet. 1983;1(8322):459-462.

38. Schain WS. Breast reconstruction. Update of psychosocial and pragmatic concerns. Cancer. 1991;68(5 Suppl):1170-1175.

39. Yang RL, Newman AS, Lin IC, et al. Trends in immediate breast reconstruction across insurance groups after enactment of breast cancer legislation. Cancer. 2013;119(13):2462-2468.

40. Albornoz CR, Bach PB, Pusic AL, et al. The influence of sociodemographic factors and hospital characteristics on the method of breast reconstruction, including microsurgery: a U.S. population-based study. Plastic and reconstructive surgery. 2012;129(5):1071-1079.

41. Alderman AK, McMahon L, Jr., Wilkins EG. The national utilization of immediate and early delayed breast reconstruction and the effect of sociodemographic factors. Plastic and reconstructive surgery. 2003;111(2):695-703; discussion 704-695. 



\section{Chapter}

\section{Analyzing Regional Differences over a Fifteen- year Trend of One-stage versus Two-staged Breast Reconstruction in 941,191 Post- Mastectomy Patients}

Parisa Kamali

Pieter G.L. Koolen

Ahmed M.S. Ibrahim

Marek A. Paul

Rieky E. Dikmans

Marc L. Schermerhorn

Bernard T. Lee

Samuel J. Lin 


\section{ABSTRACT}

\section{Background}

Implant-based reconstruction is the predominant form of breast reconstruction with two-staged tissue expander/implant approach being the most popular. Recently, directto-implant, one-stage breast reconstruction procedure has gained momentum. In this study national and regional trends across the US for the two different types of implantbased reconstructions were evaluated.

\section{Methods}

The Nationwide Inpatient Sample (NIS) database was used to extrapolate data on type of mastectomy, implant-based reconstructive technique (one-stage/two-staged), and sociodemographic and hospital variables. Differences were assessed using chi-square, impact of variables on reconstructive method was analyzed using logistic regression and trends were analyzed using Cochrane-Armitage test.

\section{Results}

Between 1998 and 2012, a total of 1,444,587 patients treated for breast cancer or at increased risk of breast cancer met the defined selection criteria. Of these, 194,377 patients underwent implant-based breast reconstruction (13.6\% one-stage and $86.4 \%$ two-staged). In both, there was a significant increase in procedures performed over time $(p<0.001)$. The highest increase in both was seen in the Northeast region of the US, and the lowest in the Southern region. When stratified into regions, analysis showed differences in socio-economic and hospital characteristics within the different regions.

\section{Conclusion}

There is an observed increase in the number of one-stage and two-staged breast reconstructions being performed. Sociodemographic and hospital factors of influence vary in the different regions of the USA. This study provides important information for clinicians and policy-makers who seek to ensure equitable and appropriate access for patient to the different types of implant-based procedures. 


\section{INTRODUCTION}

Breast cancer is the most common non-cutaneous malignancy affecting women in the United States and the second leading cause of cancer mortality. ${ }^{1}$ In 2015, an estimated 231,840 new cases of invasive breast cancer (BC) and 64,290 cases of in situ breast cancer are expected to be diagnosed in the United States alone. ${ }^{1}$ Approximately 40 to 55 percent of these women opt for simple (total) mastectomy. ${ }^{2}$ Given the significant psychosocial morbidity of this procedure and the increased quality-of-life benefits of post-mastectomy breast reconstruction, substantial efforts are devoted to optimizing these techniques. ${ }^{3-5}$ In the United States, the preferred method of post-mastectomy breast reconstruction is implant-based. ${ }^{6}$ Traditionally, implant-based breast reconstruction is performed in a two-staged fashion. In the first stage a tissue expander is placed followed by replacement of the tissue expander with a permanent implant (saline or silicone) in the second stage. ${ }^{7}$ However, due to improvements in surgical technique (the advent of skin-sparing or nipple-areola complex (NAC)-sparing mastectomies and the incorporation of acellular dermal matrices), and the increasing number of women seeking bilateral prophylactic mastectomies, immediate one-stage implantation of a permanent implant has gained popularity over the last decade. ${ }^{8-17}$ One-stage implant based breast reconstruction has allowed for immediate reconstruction of post-mastectomy defects without the need for a temporary tissue expander to expand the overlying soft tissue or a second-stage exchange procedure. ${ }^{11-15}$ Previous studies have demonstrated that sociodemographic variables such as age, income, insurance type, and geographic region can negatively impact whether a patient receives breast reconstruction. ${ }^{18-21}$ Although numerous studies have consistently reported successful clinical outcomes following either one-stage or two-staged implantbased breast reconstruction ${ }^{16}$, to date, there is a lack of large-scale studies focused on overall and regional trends in the United States. In the current study, we sought to evaluate nationwide trends in post-mastectomy one-stage and two-staged breast reconstruction rates.

\section{PATIENTS AND METHODS}

Data were obtained from the Healthcare Cost and Utilization Project Nationwide Inpatient Sample (HCUP-NIS) for the years 1998 to 2012. The HCUP-NIS is the largest allpayer inpatient database in the United States, containing data from nearly 8 million inpatient hospital stays yearly. It is designed to approximate a $20 \%$ sample of all hospital discharges, which includes all nonfederal, short-term, general, and other specialty hospitals, including public hospitals and academic institutions. This database contains a primary, and depending on the year, up to 15 additional diagnoses. 
Our cohort comprised all female patients with a primary diagnosis of breast cancer or those at increased risk of breast cancer undergoing total, subcutaneous, simple, extended and radical mastectomy (International Classification of Diseases (ICD-9), Ninth Revision; diagnosis codes 174.0 to 174.9, 233.0, v16.3, v10.3, v84.01) (Supplemental Digital Content 1 and 2). Ablative procedures were classified as either unilateral mastectomy (UM) (using ICD-9 procedure codes 85.33, 85.34, 85.41, 85.43, 85.45. 85.47), or bilateral mastectomy (BM) (using ICD-9 procedure codes 85.35, 85.36, 85.42, $85.44,85.46,85.48$ ) (Supplemental Digital Content 2). Patients with the multiple ICD-9 procedure code 85.40 ("Mastectomy") or v50.41 ("Prophylactic removal of breast") were classified as having bilateral mastectomy. Partial mastectomies as well as lumpectomy were not included in the cohort. Implant based breast reconstruction procedures were divided into two groups based on reconstruction timing; One-stage procedures were defined as any type of mastectomy with concurrent, immediate breast implant insertion (codes 85.33, 85.35, 85.53 and 85.54), whereas two-staged breast reconstruction was defined as any mastectomy with concurrent tissue expander insertion or removal (codes 85.95 and 85.96) (Supplemental Digital Content 3). Male patients, those under 18 years of age, and those of unknown gender or age were excluded. Furthermore, cases of tissue expander placement without a concurrent mastectomy were considered delayed breast reconstruction and therefore excluded from analysis from this study.

The analysed variables included age, calendar year, region, race, hospital status (teaching/non-teaching, urban/rural), hospital bed size, primary payer (Medicare, Medicaid, private insurance and other insurance types), and income quartile. These variables have previously been shown to influence the type of reconstruction and were therefore included as potential predictors. ${ }^{18,} 21$ The hospital's census region was obtained from the AHA Annual Survey of Hospitals and is defined by the U.S. Census Bureau. The states included in each region are as follows: Northeast region includes: $M E, N H, V T, M A, R I, C T, N Y N J$, and PA. Midwest region includes: $O H, I N, I L, M I, W I, M N$, $I A, M O, N D, S D, N E$, and $K S$. The South region includes: DE, MD, DC, VA, WV, NC, SC, GA, $F L, K Y, T N, A L, M S, A R, L A, O K$, and TX. The West region includes: $M T, I D, W Y, C O, N M$, $A Z, U T, N V, W A, O R, C A, A K$, and HI. Hospital teaching status is determined by the Nationwide Inpatients Sample as those hospitals with American Medical Associationapproved residency programs, or are members of the council of Teaching Hospitals, or have a ratio of full-time residents to beds of 0.25 or greater. ${ }^{22}$ Hospital bed size categories were determined by the Nationwide Inpatient Sample based on hospital beds and are specific to the hospital's location and teaching status. Small bedsize was determined as 1-49 for rural hospital, 1-99 for urban non-teaching hospital and 1-299 for urban, teaching hospital. Medium bedsize was determined as 50-99 for rural hospital, 100-199 for urban non-teaching hospital and 300-499 beds for urban teaching hospital. Large bedsize was determined as 100+ for rural hospital, 200+ for urban nonteaching hospital and 500+ for urban teaching hospital. ${ }^{22}$ Estimated household income, 
as determined by median household income for the patient's zip code, was recorded directly from the NIS database and stratified by quartile, indicating the poorest to wealthiest populations. As these estimates are updated annually, the value range varies by year (\$1- \$28,999 in 1998 and \$1-\$37,999 in 2012 for the first quartile; \$29,000$\$ 36,999$ in 1998 and $\$ 38,000-\$ 47,999$ in 2012 for the second quartile; $\$ 37,000-\$ 49,000$ in 1998 and $\$ 48,000$ to $\$ 63,999$ in 2012 for the third quartile; $>\$ 50,000$ in 1998 and $>\$ 64,000$ in 2012 for the fourth quartile). Patients' ages were divided into groups $(<39$, 40-49, 50-59 and >60). In the NIS, race is classified into similar categories as census population data (White, Black, Hispanic, Asian or Pacific Islander, Native American and other). For the analysis, Native Americans were combined into the "other" category due to limited sample sizes.

\section{Statistical Analysis}

National estimates were calculated by uniformly applying stratum weights to the discharges according to the sampled stratum from which the discharge was drawn. ${ }^{23}$ Categorical variables were compared using Chi-square analyses. Trends were analyzed using the Cochran-Armitage test. A multivariate logistic regression model was constructed to determine patient, sociodemographic and hospital characteristics associated with type of procedure. All analyses were performed using SPSS 22.0 statistical software (IBM, Armonk, NY, USA) and significance was set at $p<0.05$.

\section{RESULTS}

During the study period, a total of 1,444,587 diagnosed with breast cancer or increased risk of developing breast cancer met the defined selection criteria. In total, 941,191 patients (65\%) were treated with mastectomy (Figure 1). The proportion of women treated with mastectomy increased from $69.90 \%$ in 1998 to $81.6 \%$ in 2012 ( $p<0.001$ ). Of these, 758,739 (80.6\%) underwent unilateral mastectomy, and 183,452 (19.4\%) bilateral mastectomy. Unilateral mastectomy decreased from $92.9 \%$ in 1998 to $56.7 \%$ in 2012 ( $p<0.001$ ). Bilateral mastectomy consequently increased from $7.1 \%$ to $56.7 \%$ from 1998 to $2012(p<0.001)$ (Figure 2). 


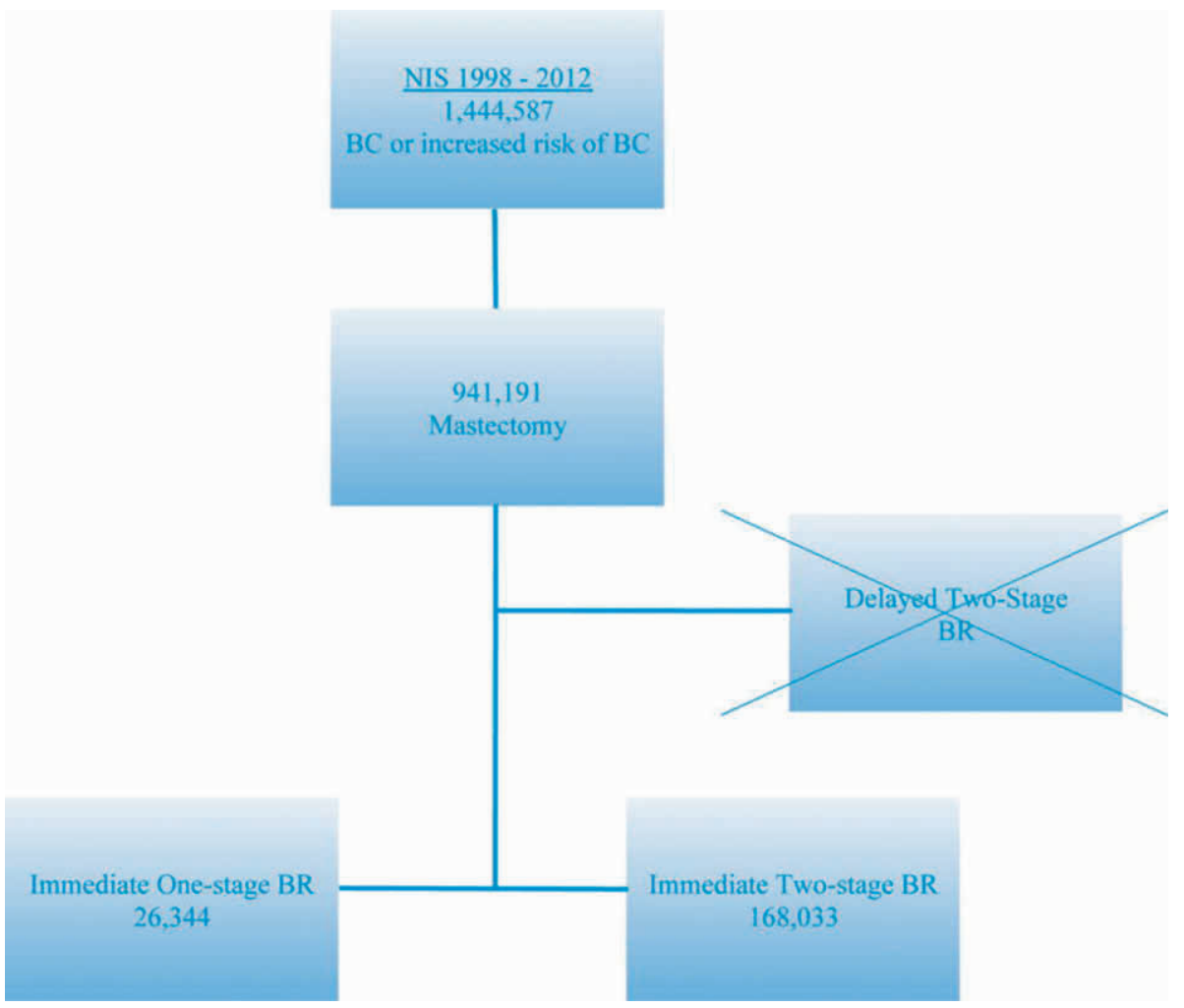

Figure 1: Inclusion patient NIS database $1998-2012$. (Breast Cancer $=$ BC, Breast Reconstruction $=B R$ )

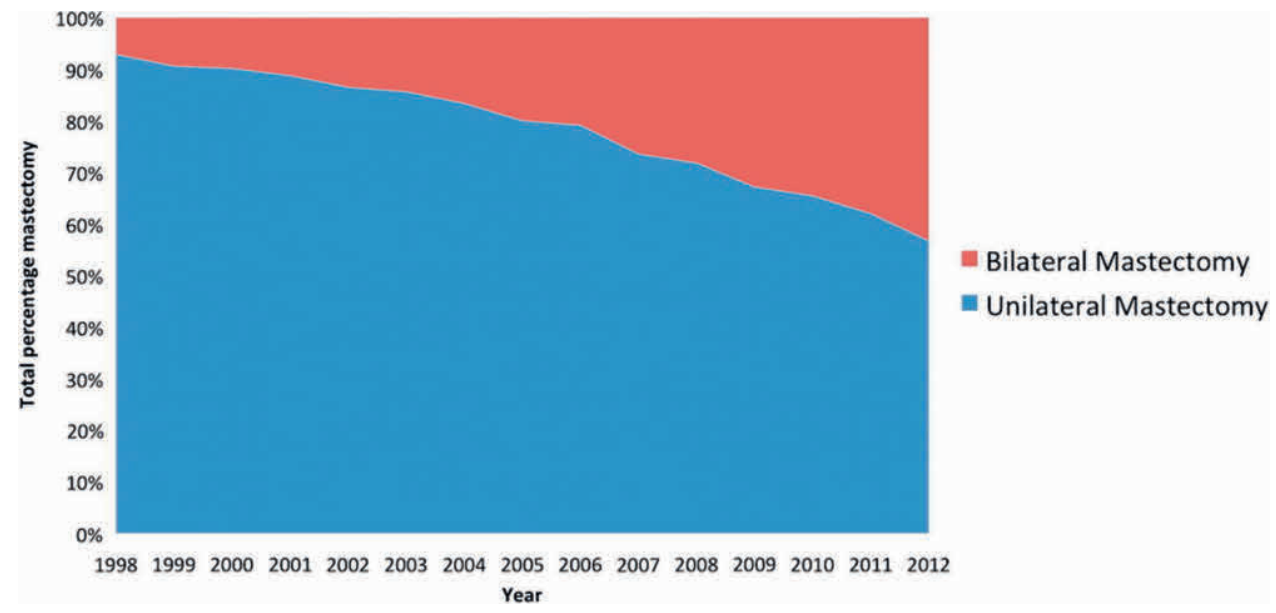

Figure 2. Trends in Mastectomy, Unilateral and Bilateral Mastectomy. (Cochrane-Armitage test for trend Unilateral Mastectomy $p<0.001$ Bilateral mastectomy $p<0.001$ ) 
A total of 194,377 (20.7\%) out of the 941,191 patients underwent immediate implantbased breast reconstruction: 26,344 (13.6\%) one-stage and 168,033 (86.4\%) two-staged (Figure 1). A flowchart with the subdivision of patients with either breast cancer or at increased risk of breast cancer, undergoing unilateral or bilateral mastectomy, followed by one-stage or two-stage breast reconstruction is shown in Supplemental Digital Content 4. In both groups, demographic characteristics were comparable; a majority of patients were Caucasian women, aged 40-49, who underwent unilateral reconstruction, were operated on at a teaching urban hospital with large bedsize, estimated to have a household income in the fourth quartile, and with private insurance (Table 1).

Table 1. Baseline characteristics by one-stage or two-staged breast reconstruction.

\begin{tabular}{|c|c|c|c|c|c|}
\hline \multirow[t]{2}{*}{ Characteristics } & \multicolumn{2}{|l|}{ One-stage BR } & \multicolumn{2}{|c|}{ Two-stage BR } & \multirow[t]{2}{*}{$p$ value } \\
\hline & $(n=26.344)$ & $(\%)$ & $(n=168$. & )$(\%)$ & \\
\hline Percentage of total & & $13.6 \%$ & & $86.4 \%$ & \\
\hline Age & & & & & 0.311 \\
\hline$<39$ years & 3563 & $13.5 \%$ & 23210 & $13.8 \%$ & \\
\hline 40-49 years & 9130 & $34.7 \%$ & 57957 & $34.5 \%$ & \\
\hline $50-59$ years & 7931 & $30.1 \%$ & 51018 & $30.4 \%$ & \\
\hline$>60$ years & 5720 & $21.7 \%$ & 35848 & $21.3 \%$ & \\
\hline Race & & & & & $<0.001$ \\
\hline Caucasian & 17873 & $67.8 \%$ & 112274 & $66.8 \%$ & \\
\hline Black & 1350 & $5.1 \%$ & 10003 & $6.0 \%$ & \\
\hline Hispanic & 955 & $3.6 \%$ & 7863 & $4.7 \%$ & \\
\hline Asian/Pacific Islander & 603 & $2.3 \%$ & 3391 & $2.0 \%$ & \\
\hline Other & 5563 & $21.1 \%$ & 34502 & $20.5 \%$ & \\
\hline Mastectomy & & & & & 0.067 \\
\hline Unilateral & 14083 & $53.5 \%$ & 90845 & $54.1 \%$ & \\
\hline Bilateral & 12261 & $46.5 \%$ & 77188 & $45.9 \%$ & \\
\hline Estimated Household Income & & & & & $<0.001$ \\
\hline First quartile & 2149 & $8.30 \%$ & 15488 & $9.40 \%$ & \\
\hline Second quartile & 4042 & $15.70 \%$ & 27298 & $16.60 \%$ & \\
\hline Third quartile & 6263 & $24.30 \%$ & 41055 & $24.90 \%$ & \\
\hline Fourth quartile & 13339 & $51.70 \%$ & 81069 & $49.20 \%$ & \\
\hline \multicolumn{6}{|l|}{ Hospital location } \\
\hline Rural & 1141 & $4.3 \%$ & 4129 & $2.7 \%$ & $<0.001$ \\
\hline Urban & 25158 & $95.70 \%$ & 163470 & $97.50 \%$ & \\
\hline Hospital teaching status & & & & & $<0.001$ \\
\hline Non-teaching & 11094 & $42.20 \%$ & 61053 & $36.40 \%$ & \\
\hline Teaching & 15205 & $57.80 \%$ & 106545 & $63.60 \%$ & \\
\hline Hospital Bedsize & & & & & $<0.001$ \\
\hline Small & 3643 & $13.90 \%$ & 19484 & $11.6 \%$ & \\
\hline Medium & 6077 & $23.10 \%$ & 34000 & $20.30 \%$ & \\
\hline Large & 16580 & $63.00 \%$ & 114115 & $68.10 \%$ & \\
\hline
\end{tabular}




\begin{tabular}{|c|c|c|c|c|c|}
\hline \multirow[t]{2}{*}{ Characteristics } & \multicolumn{2}{|l|}{ One-stage BR } & \multicolumn{2}{|c|}{ Two-stage BR } & \multirow[t]{2}{*}{$p$ value } \\
\hline & $(n=26.344)$ & $(\%)$ & ( $n=168$ & )$(\%)$ & \\
\hline Payer & & & & & $<0.001$ \\
\hline Private & 15673 & $59.5 \%$ & 101754 & $60.6 \%$ & \\
\hline Medicaid & 808 & $3.1 \%$ & 6724 & $4.0 \%$ & \\
\hline Medicare & 2489 & $9.4 \%$ & 14254 & $8.5 \%$ & \\
\hline Other & 7373 & $28.0 \%$ & 45301 & $27.0 \%$ & \\
\hline Hospital Region & & & & & $<0.001$ \\
\hline North East & 7889 & $29.9 \%$ & 49408 & $29.4 \%$ & \\
\hline Midwest & 4547 & $17.3 \%$ & 35310 & $21.0 \%$ & \\
\hline South & 7775 & $29.5 \%$ & 50216 & $29.90 \%$ & \\
\hline West & 6133 & $23.3 \%$ & 33100 & $19.7 \%$ & \\
\hline
\end{tabular}

In patients undergoing mastectomy, one-stage breast reconstruction increased from $1.7 \%$ in 1998 to $5.4 \%$ in $2012(p<0.001)$ and two-staged breast reconstruction increased from 9.9\% to 33.7\% between 1998 and 2012 ( $p<0.001$ ) (Figure 3).

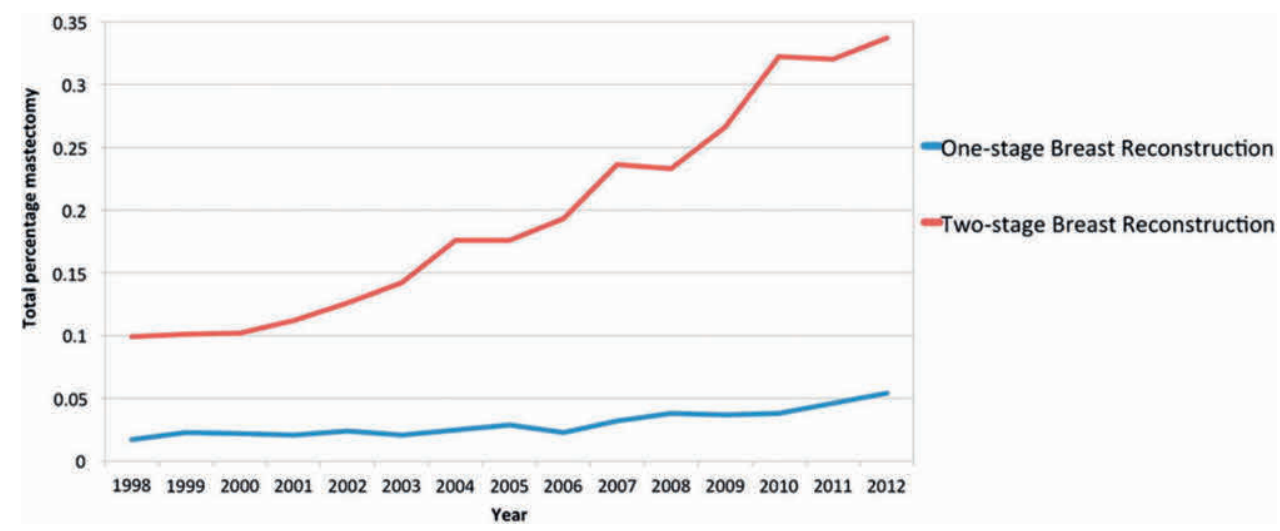

Figure 3. Post-mastectomy breast reconstruction: Trend over time in One-stage and Two-staged breast reconstruction (Cochrane-Armitage test for trend One-stage $p<0.001$ Two-staged breast reconstruction $p<0.001)$.

Figure 4 shows independent predictors of one-stage versus two-staged breast reconstructions. One-stage breast reconstruction is more likely in women undergoing bilateral mastectomy (odds ratio (OR) 1.04, $p=0.002$ ), of the "other" ethnicity type (OR $1.17, p<0.001)$, with an estimated household income in the third (OR 1.07, $p=0.016)$ and fourth quartiles (OR 1.02, $p<0.001$ ), on Medicare (OR 1.16, $p<0.001$ ) and other insurance providers (OR 1.06, $\mathrm{p}<0.001$ ), and in the West region of the USA (OR 1.09, $p<0.001)$. Two-staged breast reconstruction was more likely in women aged $>60$ years (OR 0.96, $p=0.038$ ), of Black (OR 0.94, $p=0.049$ ) and Hispanic ethnicity (OR 0.76, $p<0.001)$, at an urban (OR 0.56, $p<0.001)$, teaching hospital (OR 0.82, $p<0.001$ ) with a medium (OR 0.96, $p=0.0048$ ) and large bedsize (OR 0.75, $p<0.001$ ), on Medicaid 
insurance (OR 0.83, $p<0.001)$ and in the Midwest (OR 0.75, $p<0.001$ ) and Southern regions (OR $0.75, p<0.001$ ) of the US.

\section{Multivariate Analysis Comparing the Likelihood of Having a \\ One-stage (1) BR versus a Two-stage (0) BR $(n=194,377)$}

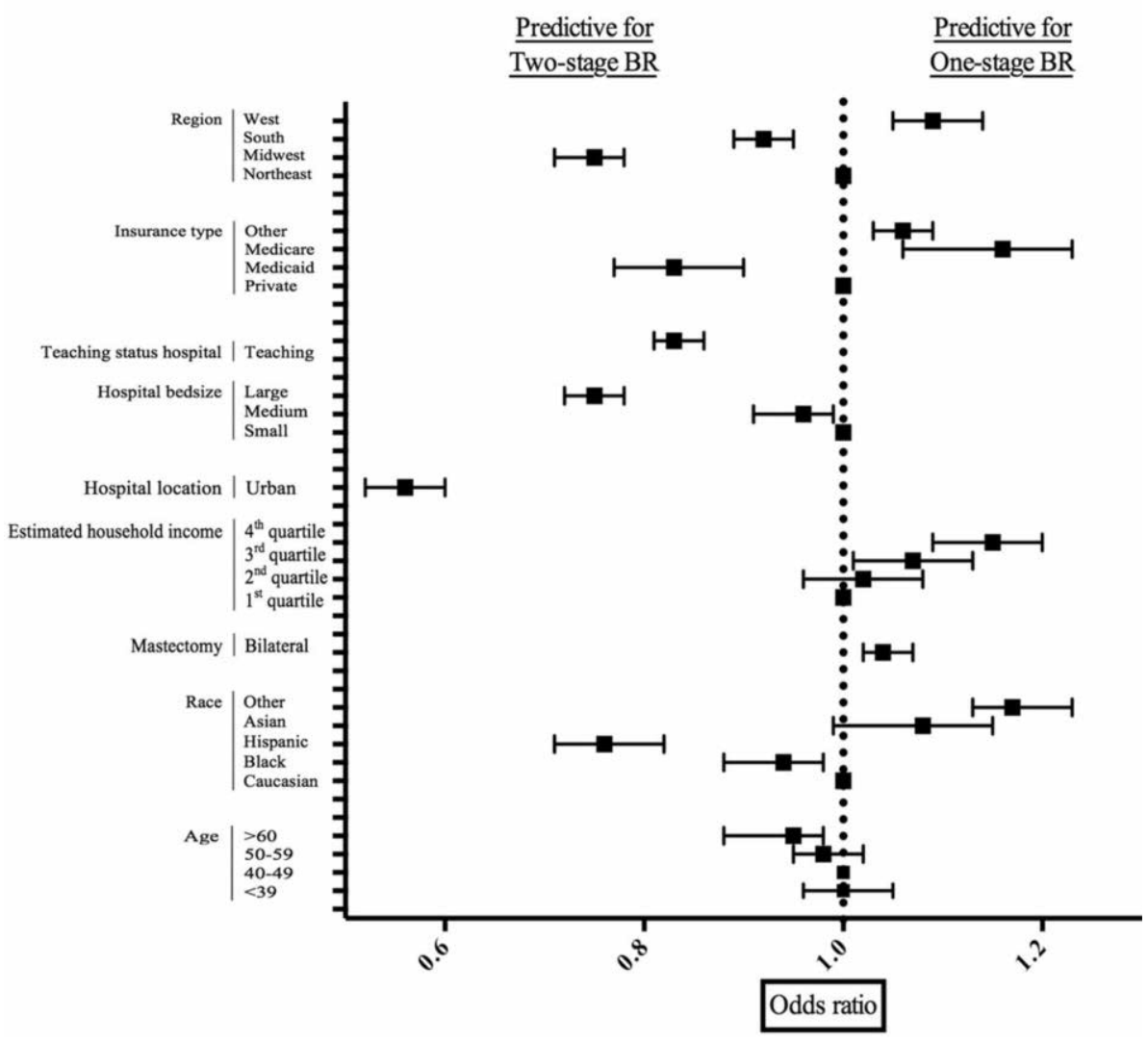

Figure 4. Multivariate Analysis Comparing the Likelihood of Having a One-stage (1) Breast Reconstruction versus Two-staged (0) Breast Reconstruction ( $n=194,377)$.

One-stage breast reconstruction: Comparison by Region

Figure 5 shows the highest percentage of baseline characteristics stratified by region. The highest increase over time in both one-stage and two-staged breast reconstruction was seen in the Northeastern region 


\begin{tabular}{|c|c|c|c|c|c|}
\hline \multicolumn{6}{|c|}{ HIGHEST PERCENTAGE IN BASELINE CHARACTERISTICS PER REGION } \\
\hline \multirow{3}{*}{$\begin{array}{c}\text { Northeast } \\
\text { Region }\end{array}$} & \multirow{9}{*}{$\begin{array}{l}\text { Age } \\
\text { Race } \\
\text { Mastectomy } \\
\text { Est. Household Income } \\
\text { Hospital location } \\
\text { Hospital teaching status } \\
\text { Hospital bedsize } \\
\text { Payer }\end{array}$} & \multicolumn{2}{|c|}{ One-stage } & \multicolumn{2}{|c|}{ Two-stage } \\
\hline & & $40-49$ & $36.8 \%$ & $40-49$ & $37.0 \%$ \\
\hline & & Caucasian & $78.2 \%$ & Caucasian & $78.2 \%$ \\
\hline & & Unilateral & $54.0 \%$ & Unilateral & $56.9 \%$ \\
\hline \multirow{13}{*}{$\begin{array}{l}\text { South } \\
\text { Region }\end{array}$} & & Fourth quartile & $61.8 \%$ & Fourth quartile & $60.8 \%$ \\
\hline & & Urban & $95.6 \%$ & Urban & $98.8 \%$ \\
\hline & & Teaching & $67.0 \%$ & Teaching & $80.5 \%$ \\
\hline & & Large & $57.2 \%$ & Large & $66.0 \%$ \\
\hline & & Private & $66.5 \%$ & Private & $65.4 \%$ \\
\hline & Age & 40-49 & $37.5 \%$ & 40-49 & $34.7 \%$ \\
\hline & Race & Other & $52.2 \%$ & Other & $50.7 \%$ \\
\hline & Mastectomy & Unilateral & $55.4 \%$ & Unilateral & $52.7 \%$ \\
\hline & Est. Household Income & Fourth quartile & $46.4 \%$ & Fourth quartile & $43.0 \%$ \\
\hline & Hospital location & Urban & $93.3 \%$ & Urban & $96.2 \%$ \\
\hline & Hospital teaching status & Teaching & $66.3 \%$ & Teaching & $70.4 \%$ \\
\hline & Hospital bedsize & Large & $68.5 \%$ & Large & $72.4 \%$ \\
\hline & Payer & Private & $59.6 \%$ & Private & $61.2 \%$ \\
\hline \multirow{8}{*}{$\begin{array}{c}\text { Midwest } \\
\text { Region }\end{array}$} & Age & $40-49$ & $33.4 \%$ & $40-49$ & $32.1 \%$ \\
\hline & Race & Caucasian & $69.0 \%$ & Caucasian & $68.5 \%$ \\
\hline & Mastectomy & Unilateral & $52.8 \%$ & Unilateral & $53.3 \%$ \\
\hline & Est. Household Income & Fourth quartile & $39.4 \%$ & Fourth quartile & $37.4 \%$ \\
\hline & Hospital location & Urban & $96.5 \%$ & Urban & $97.1 \%$ \\
\hline & Hospital teaching status & Non-teaching & $52.2 \%$ & Teaching & $54.1 \%$ \\
\hline & Hospital bedsize & Large & $63.6 \%$ & Large & $63.7 \%$ \\
\hline & Payer & Private & $53.2 \%$ & Private & $56.1 \%$ \\
\hline \multirow{8}{*}{$\begin{array}{l}\text { West } \\
\text { Region }\end{array}$} & Age & $50-59$ & $32.4 \%$ & $40-49$ & $34.1 \%$ \\
\hline & Race & Caucasian & $71.2 \%$ & Caucasian & $70.6 \%$ \\
\hline & Mastectomy & Unilateral & $52.2 \%$ & Unilateral & $52.6 \%$ \\
\hline & Est. Household Income & Fourth quartile & $58.3 \%$ & Fourth quartile & $56.3 \%$ \\
\hline & Hospital location & Urban & $96.4 \%$ & Urban & $97.7 \%$ \\
\hline & Hospital teaching status & Teaching & $96.4 \%$ & Non-teaching & $97.7 \%$ \\
\hline & Hospital bedsize & Large & $65.8 \%$ & Large & $73.3 \%$ \\
\hline & Payer & Private & $58.4 \%$ & Private & $59.4 \%$ \\
\hline
\end{tabular}

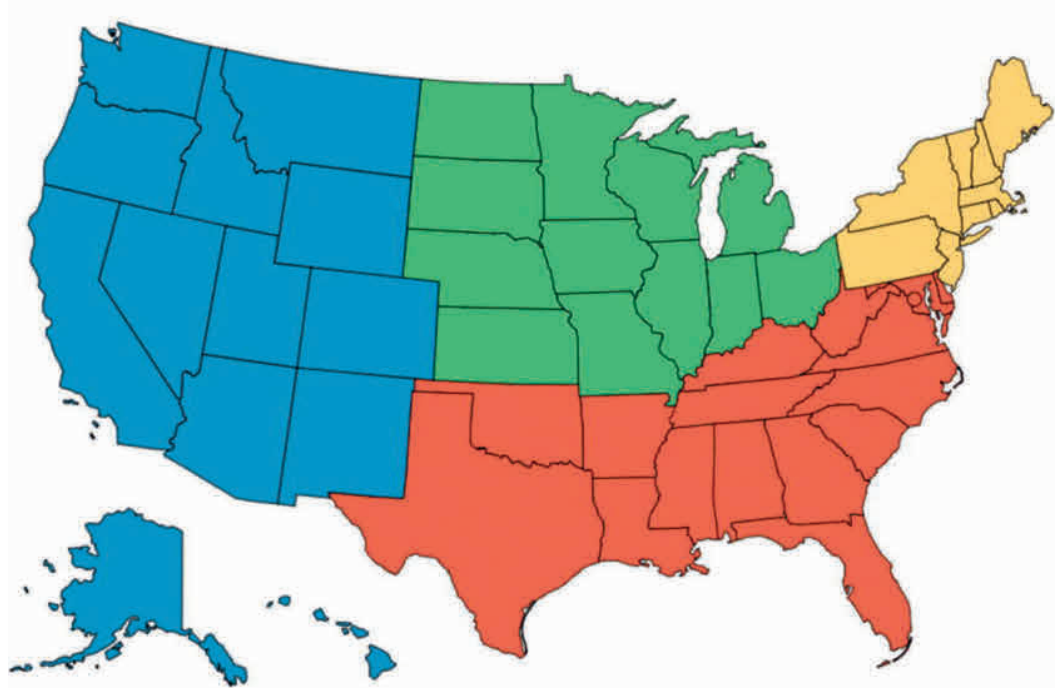

Figure 5. Highest percentage in baseline characteristics stratified per region of the United States. 
(Figure $6 a$ and b). In the Northeastern region of the USA, 57,296 (29.5\% of total) patients underwent implant based breast reconstruction of which 7,888 (13.8\%) were one-stage and 49,408 (86.2\%) were two-staged. When adjusted for sociodemographic and hospital variables, one-stage breast reconstruction was more common in patients having a bilateral mastectomy (OR 1.13, $p<0.001)$, "other" ethnicity (OR 1.66, $p<0.001$ ) and Medicare insurance (OR 1.15, p=0.012) (Figure 7a).
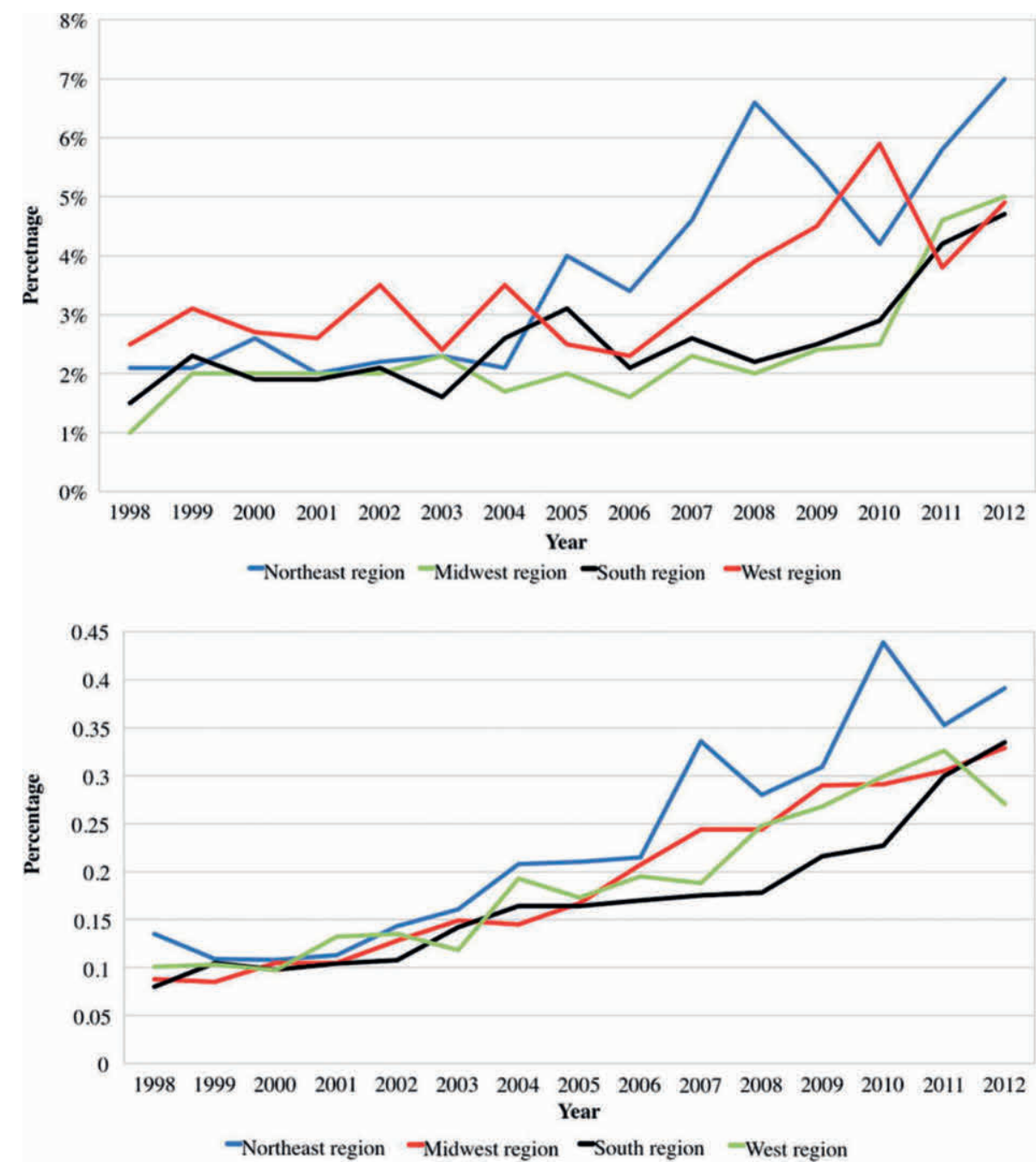

Figure 6. Regional trend over time: One-stage breast reconstruction. 6b: Regional trend over time: Twostaged breast reconstruction. 
In the Midwest region, a total of 39,857 (20.5\% of total) patients underwent implantbased reconstruction. The lowest overall number of one-stage breast reconstruction procedures was performed in this subset of patients compared to all the other regions (4,547 patients; 17.3\%) (Table 1). Eighty-eight percent of patients undergoing implant reconstruction had two-staged breast reconstruction (35,311). Associated variables for having a one-stage breast reconstruction in this region were women of Black ethnicity (OR 1.29, $p=0.007$ ), in the fourth quartile of estimated income (OR 1.19, $p=0.009$ ), and on Medicare insurance (OR 1.28, p<0.001) (Figure 7b).

57,988 ( $29.8 \%$ of total) patients underwent implant-based breast reconstruction in the Southern region. Within this patient population 7,774 (11.4\%) were one-stage and 50,214 (88.6\%) were two-staged. One-stage breast reconstructions in this region were found to be positively associated with "other" ethnicity (OR 1.16, p<0.001), in the fourth quartile of estimated income (OR 1.16, $p<0.001$ ) on Medicaid (OR 1.24, $p=0.003$ ) or Medicare (OR 1.15, $p=0.004$ ) and other insurance type (OR 1.12, $p<0.001$ )(Figure 7c).

In the West Region, of the 39,233 (20.2\% of total) patients, 6,132 patients (15.6\%) underwent one-stage and 33,100 patients (84.4\%) two-staged breast reconstruction. In this region, women undergoing one-stage breast reconstruction were more likely to be aged $>39$ (OR 1.30, $p<0.001$ ), be operated on at a teaching hospital (OR 1.33, $p<0.001$ ) and to have Medicare (OR 1.15, $p=0.023$ ) or "other" (OR 1.11, $p<0.001)$ insurance types (Figure 7d). 


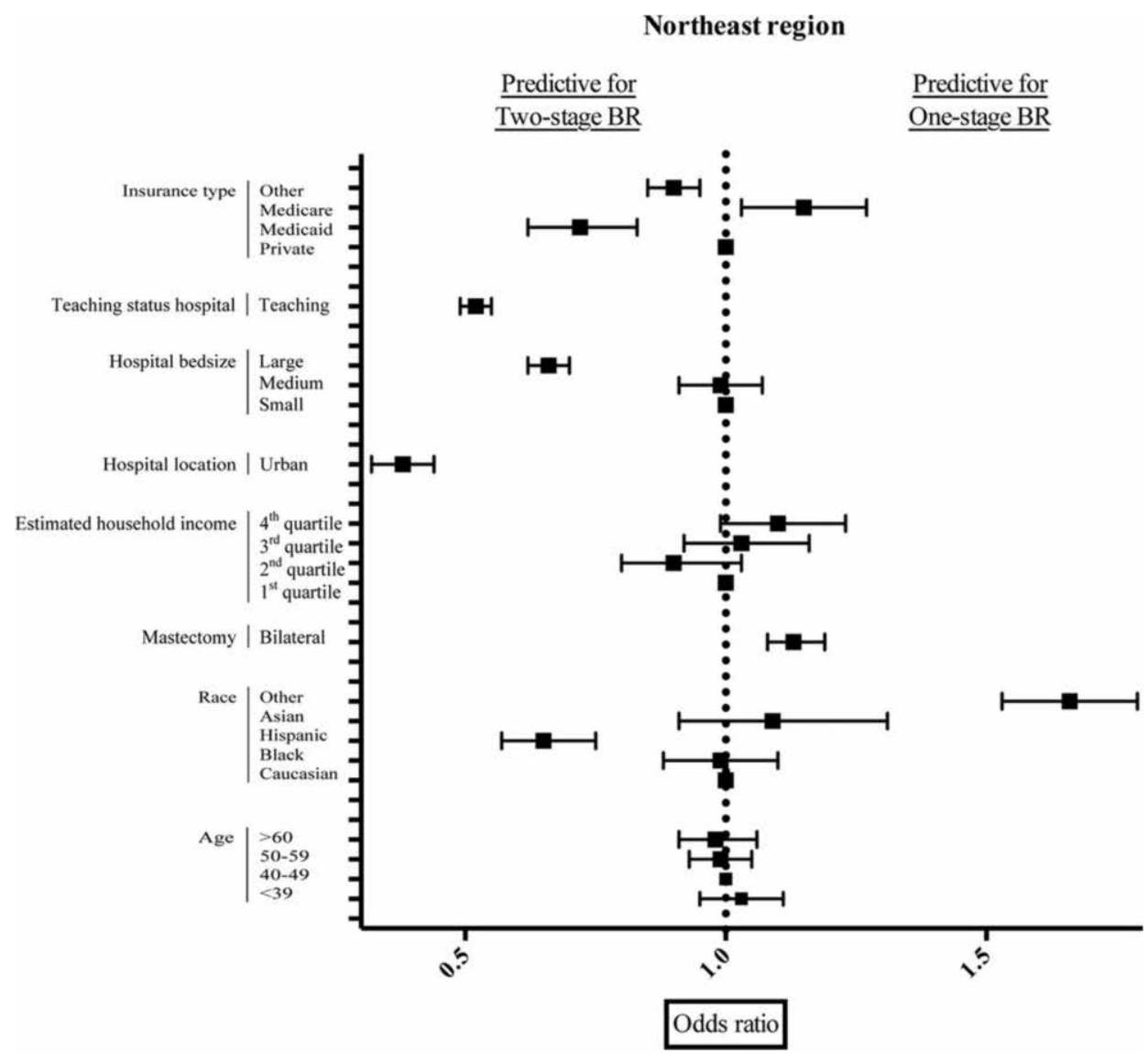

Figure 7a. Multivariate Analysis Comparing the Likelihood of Having a One-stage Breast Reconstruction versus Two-staged Breast Reconstruction Stratified by Region. 


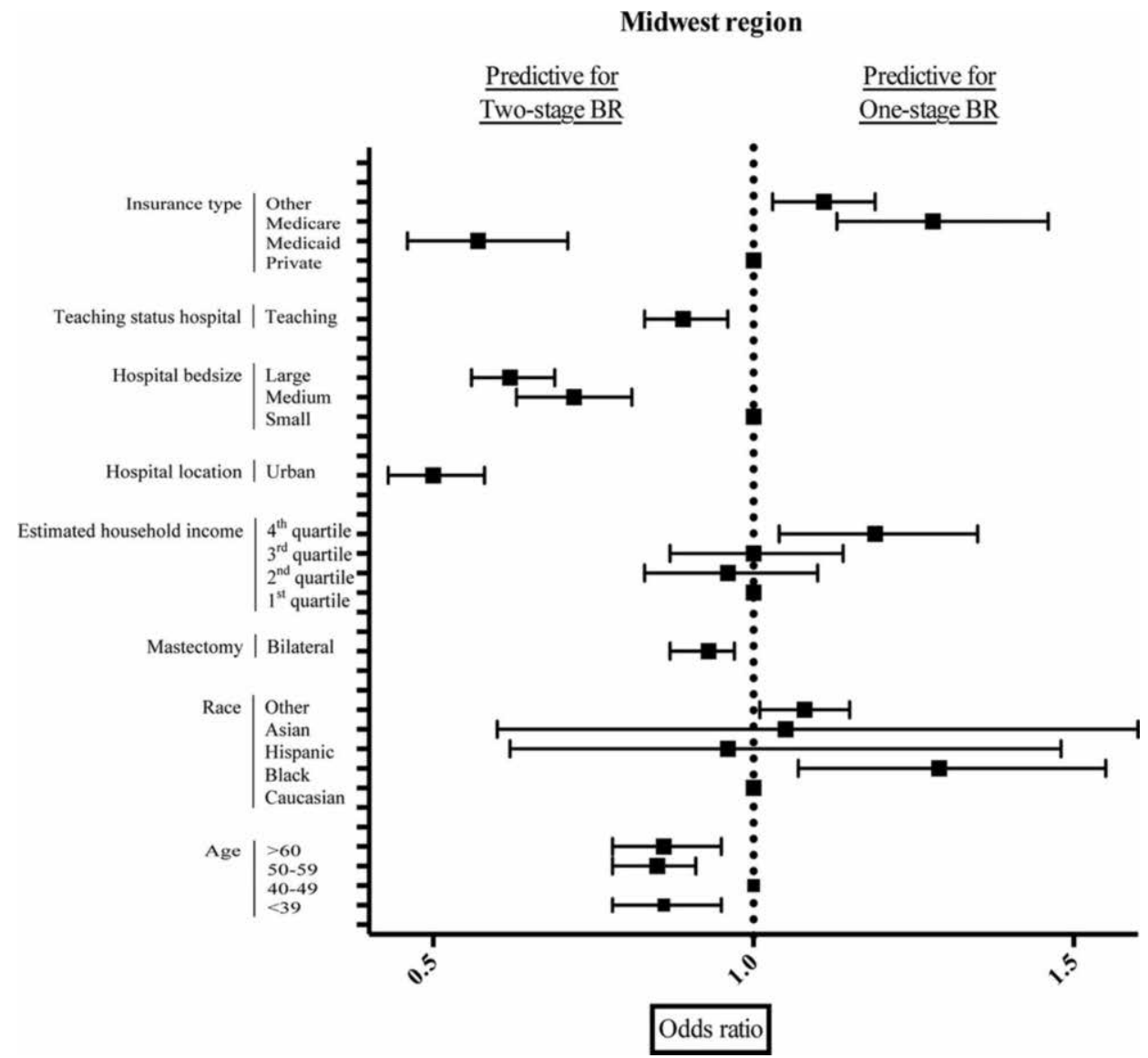

Figure 7b. Multivariate Analysis Comparing the Likelihood of Having a One-stage Breast Reconstruction versus Two-staged Breast Reconstruction Stratified by Region. 


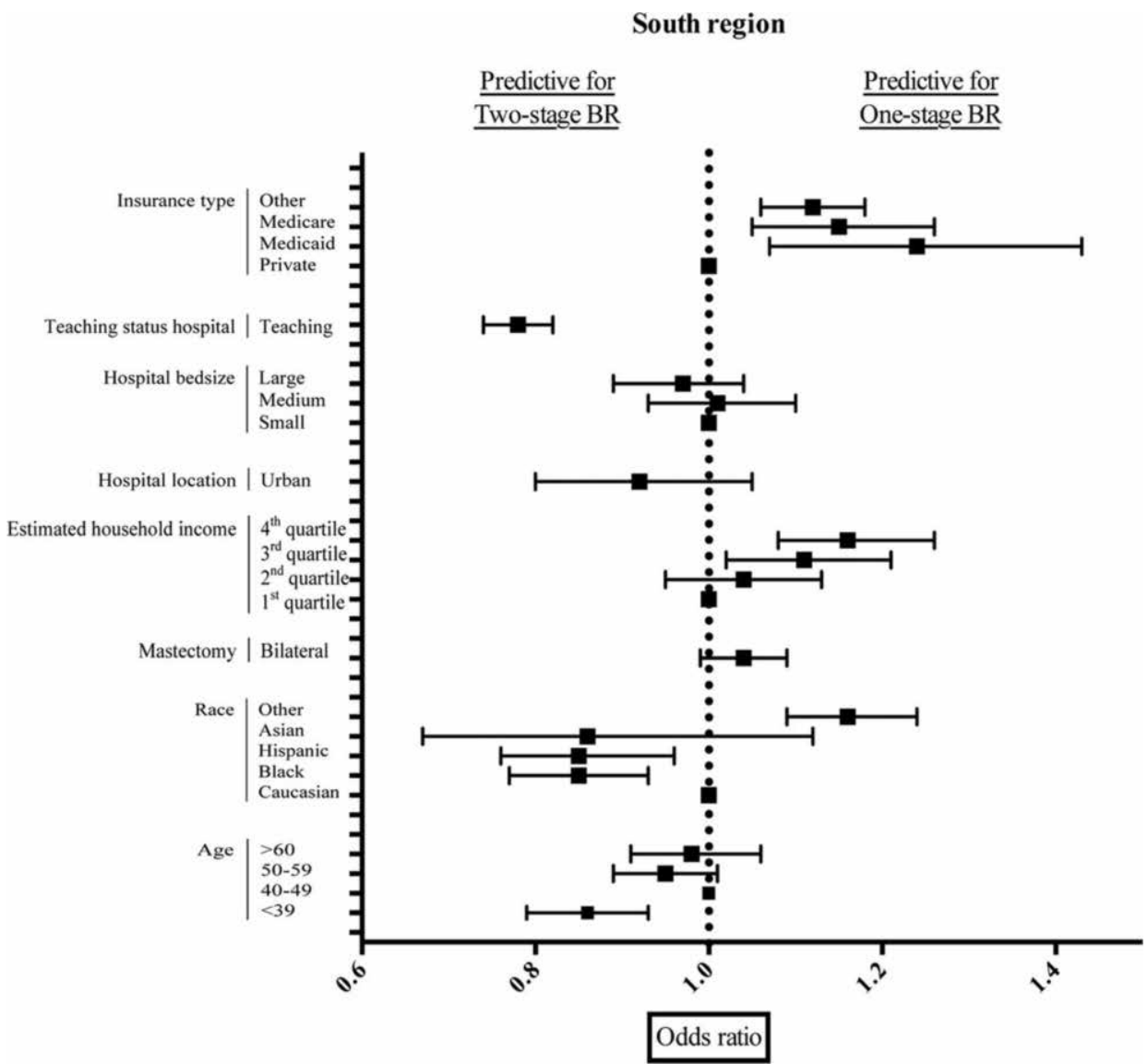

Figure 7c. Multivariate Analysis Comparing the Likelihood of Having a One-stage Breast Reconstruction versus Two-staged Breast Reconstruction Stratified by Region. 


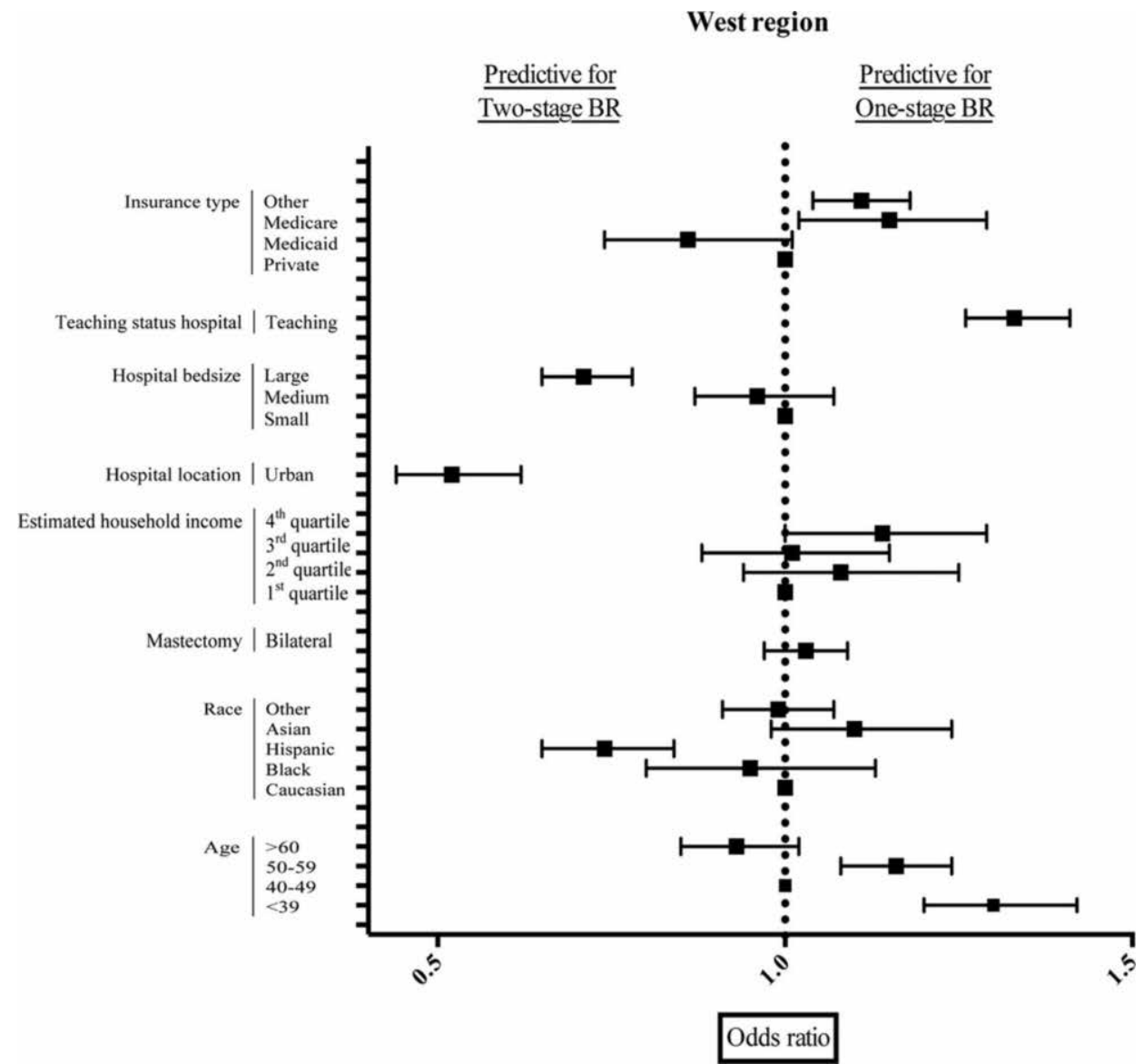

Figure 7d. Multivariate Analysis Comparing the Likelihood of Having a One-stage Breast Reconstruction versus Two-staged Breast Reconstruction Stratified by Region.

\section{DISCUSSION}

The current standard of care in implant-based breast reconstruction is the two-staged expander/implant approach. ${ }^{24}$ However, over the years, there has been increased interest in performing single-stage breast reconstruction following mastectomy. ${ }^{24}$ Previous studies have demonstrated inconsistent outcomes comparing one-stage to two-staged implant-based breast reconstructions. ${ }^{25-30}$ This analysis of over 190,000 patients is a significant large-scale, multi-institutional study evaluating national and regional trends in post-mastectomy implant-based breast reconstruction.

Our data showed an increase in both one-stage and two-staged breast reconstruction during the study period. Although one-stage breast reconstruction has gained momentum over the last decade, we found that two-staged breast reconstruction 
increased at a faster rate over the same time period (Figure 3). The largest increase in both types of implant based breast reconstruction was observed in the Northeast region of the USA (Figure 6a and 6b). In contrast, the Southern region showed the smallest increase in both types of implant based breast reconstruction (Figure 6a and 6b). A possible contributing factor for this finding may be the distribution of plastic surgeons and access to reconstruction following mastectomy. Wexelman et al. reported that accessibility to plastic surgeons was not evenly distributed throughout the United States, with states in the Northeast region of the country having a higher number of plastic surgeons compared to the West region. ${ }^{31}$ Moreover, Albonorz et al. reported that patients from the West, Midwest, and South were more likely to undergo implant-based reconstruction compared to autologous reconstruction than those in the Northeast. ${ }^{18}$ In addition to regional differences in training and preferences of plastic surgeons, the availability of surgeons who perform complex reconstruction differs from state to state.

Mastectomy followed by implant-based breast reconstruction is gaining in popularity. 6,32 Women with breast cancer or at greater risk of developing breast cancer are increasingly choosing mastectomy over breast-conserving surgery and bilateral mastectomy over unilateral, both for prophylaxis and for cancer treatment. ${ }^{6,}$ 33-36 Our analysis showed that reconstructive method varied depending on the laterality of the mastectomy. We found that patients undergoing one-stage breast reconstruction have higher odds of bilateral mastectomy (Figure 4). Interestingly, when stratified into regions, this was only seen in the Northeast (Figure 7a-d). In 1998, the Women's Health and Cancer Rights Act mandated insurance coverage of reconstruction procedures but had little impact on access and disparities in the years that ensued. More recently, the social and clinical environment surrounding management of locoregional breast cancer has changed. In line with the literature, our results showed a significant increase in bilateral mastectomy and a significant decrease in unilateral mastectomy over the years (Figure 2). ${ }^{37-41}$ Albonorz et al. reported an increase in implant use of 200 percent between 1998 and 2008. ${ }^{6}$ Although the reason for this is multifactorial, they hypothesized that a change in mastectomy patterns, that is, a rise in the number of bilateral mastectomies, as an important contributor.

Sociodemographic variables and hospital features have been previously shown to be important predictors on whether or not women choose to undergo breast reconstruction. ${ }^{19}, 20,42$ These factors were evaluated to determine whether they influence decision making for the type of implant-based breast reconstruction performed. Although our analyses show that one-stage reconstruction was relatively more likely to be performed in rural non-teaching hospitals, the vast majority of implant-based reconstructions are still being performed in urban teaching hospitals. In general, teaching hospitals are large tertiary or specialized centers with the ability to perform complex procedures because of economic and human resources available at these centers typically. 
Significant differences in health care access between rural and urban areas exist. Reluctance to seek health care in rural areas is based on cultural and financial constraints, often compounded by a scarcity of services, a lack of trained physicians and insufficient public transport. In general, rural residents have been found to be in poorer health owing to the difficulty in attracting and retaining physicians, and maintaining health services on a par with their urban counterparts. ${ }^{46}$ Access to comprehensive and quality healthcare services is difficult for socioeconomically disadvantaged groups in rural regions. Substantial racial/ethnic health disparities exist in the United States. Although the populations of racial and ethnic minorities are growing at a rapid pace, large-scale community-based surveys and surveillance systems designed to monitor the health status of minority populations are limited. More residents in these minority populations do not have health-care coverage and do not see a doctor because of cost. ${ }^{47}$ Little data are available on how race/ethnicity, insurance, and socioeconomic status (SES) interrelate to influence breast cancer treatment and type of reconstruction.

A set of sociodemographic variables were identified that represent disparities and serve as targets for future educational or legislative initiatives. These include payer and socioeconomic status. For example, on a national level we showed that the type of insurance payer impacts the type of reconstruction. Patients with Medicare insurance and other insurance carriers were more likely to receive a one-stage breast reconstruction compared to private insurance. A recent Dutch study found lower costs with single-stage breast reconstruction than with other approaches, including TE reconstruction. ${ }^{43}$ Similarly, Sing et al. showed a consistent trend toward lower costs associated with immediate onestage reconstruction, although the differences rarely achieved significance. ${ }^{2}$

There are several limitations worth noting because of the administrative nature of the NIS. First, the NIS is based upon hospital billing data, and therefore lacks data on clinical characteristics such as cancer stage and histology, family history of breast cancer, and other information that can influence the decision-making process for the type of mastectomy and reconstruction chosen. We were unable to identify operative and pathologic findings associated with procedures, limiting our ability to adjust for operative complexity. The NIS does not provide information on whether a plastic surgeon or a general surgeon performed the breast reconstructive procedure. Furthermore, the NIS uses ICD-9 procedure codes. McLaughlin et al. et al have previously demonstrated that bilateral procedures in big state databases are associated with undercoding. ${ }^{44}$ Another is that it lacks longitudinal information (i.e. a single individual cannot be tracked across multiple hospitalizations). Also, complications occurring after discharge are unfortunately not included in the NIS database. Therefore, NIS-based studies are limited to short-term, same-hospitalization outcomes. The underutilization of bilateral procedure coding might be because some insurance plans do not provide reimbursements or that the procedure is not clear to the coder. ${ }^{44}$ Finally, associations identified using large databases need to be carefully considered, as there is potential for overanalysis. Despite these limitations, the NIS enables us to study 
national and regional trends and patterns of care and therefore provide a general overview.

\section{CONCLUSION}

The current study examines the changes in implant based breast surgery in the United States over the last 15 years. Overall mastectomy rates have increased significantly. Although both one and two stage implant based techniques showed a significant rise we observed a significantly higher increase in two-staged procedures, both nationally as regionally. Furthermore, our analysis suggests that differences in sociodemographic and hospital variables affect the type of procedure performed in the different regions in the USA. Reasons for this can be multifactorial. Beyond patient and physician preference, differences in plastic surgeon specialty centers (e.g. rural/urban, teaching/nonteaching), and increased number of bilateral mastectomies, may have contributed to the higher number of the two-staged procedures. More information and further investigation is needed on patient and physician decision making to more comprehensively understand contemporary preferences in the different regions. The observations of our current study regarding trends over time and geographic variability provides valuable information for clinicians and policy-makers who seek to ensure equitable and appropriate access for patients to the different types of implant-based procedures. 


\section{TABLE AND FIGURE LEGEND}

Table 1. Baseline characteristics by one-stage or two-staged breast reconstruction.

Figure 1. Inclusion patient NIS database 1998 - 2012. (Breast Cancer $=$ BC, Breast Reconstruction $=\mathrm{BR}$ )

Figure 2. Trends in Mastectomy, Unilateral and Bilateral Mastectomy. (CochraneArmitage test for trend Unilateral Mastectomy $p<0.001$ Bilateral mastectomy $p<0.001$ )

Figure 3. Post-mastectomy breast reconstruction: Trend over time in One-stage and Two-staged breast reconstruction (Cochrane-Armitage test for trend One-stage $p<0.001$ Two-staged breast reconstruction $\mathrm{p}<0.001)$.

Figure 4. Multivariate Analysis Comparing the Likelihood of Having a One-stage (1) Breast Reconstruction versus Two-staged (0) Breast Reconstruction ( $n=194,377)$.

Figure 5. Highest percentage in baseline characteristics stratified per region of the United States.

Figure 6a. Regional trend over time: One-stage breast reconstruction.

Figure 6b. Regional trend over time: Two-staged breast reconstruction.

Figure 7 (a-d). Multivariate Analysis Comparing the Likelihood of Having a One-stage Breast Reconstruction versus Two-staged Breast Reconstruction Stratified by Region.

Supplemental Digital Content 1. Inclusion Criteria for the Study Based on ICD-9 Diagnosis Codes.

Supplemental Digital Content 2. Inclusion Criteria Ablative Procedures Based on ICD-9 Procedure Codes.

Supplemental Digital Content 3. Classification of Reconstructive Procedure Based on ICD-9 Procedure Codes.

Supplemental Digital Content 4. Flowchart of patients diagnosed with breast cancer or at increased risk of developing breast cancer undergoing unilateral or bilateral mastectomy, followed by one-stage or two-stage breast reconstruction. 


\section{REFERENCES}

1. Cancer facts \& figures. American Cancer Society. Available at: http://www.cancer.org/acs/groups/content/ @editorial/documents/document/acspc-044552.pdf Accessed April 8, 2015.

2. Singh, N., Reaven, N. L., Funk, S. E. Immediate 1-stage vs. tissue expander postmastectomy implant breast reconstructions: a retrospective real-world comparison over 18 months. Journal of plastic, reconstructive \& aesthetic surgery : JPRAS 2012;65:917-923.

3. Miller, M. J. Immediate breast reconstruction. Clinics in plastic surgery 1998;25:145-156.

4. Al-Ghazal, S. K., Sully, L., Fallowfield, L., Blamey, R. W. The psychological impact of immediate rather than delayed breast reconstruction. European journal of surgical oncology : the journal of the European Society of Surgical Oncology and the British Association of Surgical Oncology 2000;26:17-19.

5. Stevens, L. A., McGrath, M. H., Druss, R. G., Kister, S. J., Gump, F. E., Forde, K. A. The psychological impact of immediate breast reconstruction for women with early breast cancer. Plastic and reconstructive surgery 1984;73:619-628.

6. Albornoz, C. R., Bach, P. B., Mehrara, B. J., et al. A paradigm shift in U.S. Breast reconstruction: increasing implant rates. Plastic and reconstructive surgery 2013;131:15-23.

7. Serletti, J. M., Fosnot, J., Nelson, J. A., Disa, J. J., Bucky, L. P. Breast reconstruction after breast cancer. Plastic and reconstructive surgery 2011;127:124e-135e.

8. Wagner, J. L., Fearmonti, R., Hunt, K. K., et al. Prospective evaluation of the nipple-areola complex sparing mastectomy for risk reduction and for early-stage breast cancer. Annals of surgical oncology 2012;19:1137-1144.

9. Gerber, B., Krause, A., Dieterich, M., Kundt, G., Reimer, T. The oncological safety of skin sparing mastectomy with conservation of the nipple-areola complex and autologous reconstruction: an extended follow-up study. Annals of surgery 2009;249:461-468.

10. Mallon, P., Feron, J. G., Couturaud, B., et al. The role of nipple-sparing mastectomy in breast cancer: a comprehensive review of the literature. Plastic and reconstructive surgery 2013;131:969-984.

11. D'Souza, N., Darmanin, G., Fedorowicz, Z. Immediate versus delayed reconstruction following surgery for breast cancer. The Cochrane database of systematic reviews 2011:CD008674.

12. Ganske, I., Verma, K., Rosen, H., Eriksson, E., Chun, Y. S. Minimizing complications with the use of acellular dermal matrix for immediate implant-based breast reconstruction. Annals of plastic surgery 2013;71:464-470.

13. Ho, G., Nguyen, T. J., Shahabi, A., Hwang, B. H., Chan, L. S., Wong, A. K. A systematic review and metaanalysis of complications associated with acellular dermal matrix-assisted breast reconstruction. Annals of plastic surgery 2012;68:346-356.

14. Vardanian, A. J., Clayton, J. L., Roostaeian, J., et al. Comparison of implant-based immediate breast reconstruction with and without acellular dermal matrix. Plastic and reconstructive surgery 2011;128:403e-410e.

15. Venturi, M. L., Mesbahi, A. N., Boehmler, J. H. t., Marrogi, A. J. Evaluating sterile human acellular dermal matrix in immediate expander-based breast reconstruction: a multicenter, prospective, cohort study. Plastic and reconstructive surgery 2013;131:9e-18e.

16. Susarla, S. M., Ganske, I., Helliwell, L., Morris, D., Eriksson, E., Chun, Y. S. Comparison of clinical outcomes and patient satisfaction in immediate single-stage versus two-stage implant-based breast reconstruction. Plastic and reconstructive surgery 2015;135:1e-8e.

17. Rusby, J. E., Smith, B. L., Gui, G. P. Nipple-sparing mastectomy. The British journal of surgery 2010;97:305-316.

18. Albornoz, C. R., Bach, P. B., Pusic, A. L., et al. The influence of sociodemographic factors and hospital characteristics on the method of breast reconstruction, including microsurgery: a U.S. population-based study. Plastic and reconstructive surgery 2012;129:1071-1079.

19. Reuben, B. C., Manwaring, J., Neumayer, L. A. Recent trends and predictors in immediate breast reconstruction after mastectomy in the United States. American journal of surgery 2009;198:237-243. 
20. Alderman, A. K., Hawley, S. T., Janz, N. K., et al. Racial and ethnic disparities in the use of postmastectomy breast reconstruction: results from a population- based study. Journal of clinical oncology : official journal of the American Society of Clinical Oncology 2009;27:5325-5330.

21. Alderman, A. K., McMahon, L., Jr., Wilkins, E. G. The national utilization of immediate and early delayed breast reconstruction and the effect of sociodemographic factors. Plastic and reconstructive surgery 2003;111:695-703; discussion 704-695.

22. U.S. Department of Health \& Human Services. Agency for Healthcare Research and Quality (HCUP). NIS description of data elements. Available at: http://www.hcup-us.ahrq.gov/db/vars/hosp_bedsize /nisnote.jsp Accessed April 8, 2015.

23. U.S. Department of Health \& Human Services. Agency for Healthcare Research and Quality (HCUP). NIS description of data elements. Available at: https://www.hcup-us.ahrq.gov/db/nation/nis/trendwghts.jsp Accessed April 8, 2015.

24. Wink, J. D., Fischer, J. P., Nelson, J. A., Serletti, J. M., Wu, L. C. Direct-to-implant breast reconstruction: an analysis of 1612 cases from the ACS-NSQIP surgical outcomes database. Journal of plastic surgery and hand surgery 2014;48:375-381.

25. Roostaeian, J., Sanchez, I., Vardanian, A., et al. Comparison of immediate implant placement versus the staged tissue expander technique in breast reconstruction. Plastic and reconstructive surgery 2012;129:909e-918e.

26. Blechman, K. M., Karp, N. S., Levovitz, C., et al. The lateral inframammary fold incision for nipple-sparing mastectomy: outcomes from over 50 immediate implant-based breast reconstructions. The breast journal 2013;19:31-40.

27. Gui, G. P., Kadayaprath, G., Tan, S. M., et al. Long-term quality-of-life assessment following one-stage immediate breast reconstruction using biodimensional expander implants: the patient's perspective. Plastic and reconstructive surgery 2008;121:17-24.

28. Petersen, A., Eftekhari, A. L., Damsgaard, T. E. Immediate breast reconstruction: a retrospective study with emphasis on complications and risk factors. Journal of plastic surgery and hand surgery 2012;46:344-348.

29. Eriksen, C., Lindgren, E. N., Frisell, J., Stark, B. A prospective randomized study comparing two different expander approaches in implant-based breast reconstruction: one stage versus two stages. Plastic and reconstructive surgery 2012;130:254e-264e.

30. Cassileth, L., Kohanzadeh, S., Amersi, F. One-stage immediate breast reconstruction with implants: a new option for immediate reconstruction. Annals of plastic surgery 2012;69:134-138.

31. Wexelman, B., Schwartz, J. A., Lee, D., Estabrook, A., Ma, A. M. Socioeconomic and geographic differences in immediate reconstruction after mastectomy in the United States. The breast journal 2014;20:339-346.

32. Cemal, Y., Albornoz, C. R., Disa, J. J., et al. A paradigm shift in U.S. breast reconstruction: Part 2. The influence of changing mastectomy patterns on reconstructive rate and method. Plastic and reconstructive surgery 2013;131:320e-326e.

33. Soran, A., Kamali Polat, A., Johnson, R., McGuire, K. P. Increasing trend of contralateral prophylactic mastectomy: what are the factors behind this phenomenon? The surgeon : journal of the Royal Colleges of Surgeons of Edinburgh and Ireland 2014;12:316-322.

34. Pesce, C. E., Liederbach, E., Czechura, T., Winchester, D. J., Yao, K. Changing surgical trends in young patients with early stage breast cancer, 2003 to 2010: a report from the National Cancer Data Base. Journal of the American College of Surgeons 2014;219:19-28.

35. Mahmood, U., Hanlon, A. L., Koshy, M., et al. Increasing national mastectomy rates for the treatment of early stage breast cancer. Annals of surgical oncology 2013;20:1436-1443.

36. Kummerow, K. L., Du, L., Penson, D. F., Shyr, Y., Hooks, M. A. Nationwide trends in mastectomy for earlystage breast cancer. JAMA surgery 2015;150:9-16.

37. Habermann, E. B., Thomsen, K. M., Hieken, T. J., Boughey, J. C. Impact of availability of immediate breast reconstruction on bilateral mastectomy rates for breast cancer across the United States: data from the nationwide inpatient sample. Annals of surgical oncology 2014;21:3290-3296. 
38. Tuttle, T. M., Habermann, E. B., Grund, E. H., Morris, T. J., Virnig, B. A. Increasing use of contralateral prophylactic mastectomy for breast cancer patients: a trend toward more aggressive surgical treatment. Journal of clinical oncology : official journal of the American Society of Clinical Oncology 2007;25:5203-5209.

39. Tuttle, T. M., Jarosek, S., Habermann, E. B., et al. Increasing rates of contralateral prophylactic mastectomy among patients with ductal carcinoma in situ. Journal of clinical oncology : official journal of the American Society of Clinical Oncology 2009;27:1362-1367.

40. van Sprundel, T. C., Schmidt, M. K., Rookus, M. A., et al. Risk reduction of contralateral breast cancer and survival after contralateral prophylactic mastectomy in BRCA1 or BRCA2 mutation carriers. British journal of cancer 2005;93:287-292.

41. Yao, K., Stewart, A. K., Winchester, D. J., Winchester, D. P. Trends in contralateral prophylactic mastectomy for unilateral cancer: a report from the National Cancer Data Base, 1998-2007. Annals of surgical oncology 2010;17:2554-2562.

42. Polednak, A. P. Geographic variation in postmastectomy breast reconstruction rates. Plastic and reconstructive surgery 2000;106:298-301.

43. Damen, T. H., Wei, W., Mureau, M. A., et al. Medium-term cost analysis of breast reconstructions in a single Dutch centre: a comparison of implants, implants preceded by tissue expansion, LD transpositions and DIEP flaps. Journal of plastic, reconstructive \& aesthetic surgery : JPRAS 2011;64:1043-1053.

44. McLaughlin, C. C., Lillquist, P. P., Edge, S. B. Surveillance of prophylactic mastectomy: trends in use from 1995 through 2005. Cancer 2009;115:5404-5412. 



\title{
Chapter
}

\section{National and Regional Differences in 32,248 Post-Mastectomy Donor Sites for Breast Reconstruction Patients Using the Updated National Inpatient Survey}

\author{
Parisa Kamali \\ Marek A. Paul \\ Ahmed M. S. Ibrahim \\ Pieter G. L. Koolen \\ Winona Wu \\ Marc L. Schermerhorn \\ Bernard T. Lee \\ Samuel J. Lin
}




\section{ABSTRACT}

\section{Background}

The incidence of breast cancer (BC) cases has increased significantly. The number of breast reconstruction (BR) procedures performed has mirrored this trend. Although implant-only procedures remain the most commonly used type of immediate BR, autologous techniques involving donor sites account for approximately $20 \%$. The aim of this study was to assess national and regional trends in different types of autologous BR.

\section{Methods}

Using the Nationwide Inpatient Sample database (2008 to 2012), data on BC and mastectomy rates, type of autologous BR and sociodemographics were obtained and analyzed. Furthermore, national and regional trends over time for autologous BR were plotted and analyzed.

\section{Results}

A total of 427,272 patients diagnosed with BC or at increased risk of BC were included in the study. 343,163 patients (80.3\%) underwent mastectomy and within this group 148,700 patient (43.3\%) underwent IBR. Of these, 32,249 patients (21.7\%) underwent an autologous BR (not solely implant based) and 118,258 (78.3\%) implant-based BR. Most autologous BR's were performed in the Southern region (37.4\%). When stratified into flap types, most pTRAM, fTRAM, and other flaps were performed in the Northeast region, while most DIEP and LD flaps were performed in the Southern region. Subgroup analysis demonstrated a significant increasing trend for both LD and DIEP flaps, both nationally $(p<0.001)$ and regionally $(p<0.001)$. Pedicled TRAM and free TRAM reconstructions decreased significantly both on national and regional level.

\section{Conclusion}

Autologous BR demonstrated a significant positive trend over time in the Southern region $(p<0.001)$. DIEP and LD flaps increased significantly over time, both nationally and regionally. 


\section{INTRODUCTION}

In 2015, approximately 231,840 new cases of invasive breast cancer and 60,390 new cases of in-situ breast cancer are expected to be diagnosed in women in the United States. ${ }^{1}$ Nearly 35 to 40 percent of these patients will be treated with mastectomy. ${ }^{2}$ Despite the demonstrated benefits of immediate breast reconstruction after mastectomy on body image, self-esteem, ${ }^{3-5}$ sexuality, and quality of life, ${ }^{3,6-8}$ less then 25 percent of mastectomy patients opt for immediate breast reconstruction (IBR). ${ }^{9-14}$ On the other hand, advances in breast reconstructive techniques have broadened the postmastectomy reconstruction choices for women. With implant-only procedures remaining the most commonly used type of IBR, autologous techniques still account for approximately $20 \%$ of breast reconstruction. ${ }^{15}$

Besides implant-only based breast reconstruction, autologous breast reconstructive procedures have evolved into a full range of techniques over the last decades; popular options include the latissimus dorsi (LD) flap, the pedicled transverse rectus abdominis muscle (pTRAM) flap, the free transverse rectus abdominis muscle (fTRAM) flap, deep inferior epigastric perforator (DIEP) flap, the superficial inferior epigastric artery (SIEA) flap and the gluteal artery perforator (GAP) flap. ${ }^{16}$ Factors contributing to this diversification may include improved cooperation between oncologic and reconstructive surgeons, fewer contraindications for immediate breast reconstruction, improved outcomes of autologous reconstruction, and an increased awareness amongst patients regarding their reconstructive options. ${ }^{17-19}$

Although numerous studies have reported successful clinical outcomes following autologous flap breast reconstruction, to date, there is a lack of large-scale studies focused on overall and regional trends over time in the United States. The aim of this study is to (1) analyze the nationwide trend over time in autologous breast reconstruction, (2) examine regional trends over time for the different type of autologous flaps, (3) determine the distribution of potential contributing patient and socio-economic factors.

\section{PATIENT AND METHODS}

Patient data were obtained from the Healthcare Cost and Utilization Project (HCUP) Nationwide Inpatient Sample (NIS) database for the years 2008 to 2012. The NIS database is the largest all-payer inpatient care database in the United States, containing data from over 1000 hospitals and more than 8 million hospital stays annually. ${ }^{20}$ It is designed to approximate a $20 \%$ sample of all hospital discharges, which includes all nonfederal, short-term, general, and other specialty hospitals, including public hospitals and academic institutions. In 2008, the International Classification of Diseases, Ninth Revision (ICD-9) reconstructive procedural coding classified autologous breast 
reconstruction into individual flap types; pedicled latissimus dorsi (pLD), pedicled transverse rectus abdominis myocutaneous (pTRAM), free transverse rectus abdominis myocutaneous (fTRAM), deep inferior epigastric perforator (DIEP), superficial inferior epigastric artery (SIEA), and gluteal artery perforator (GAP) flaps. ${ }^{21}$ For the purposes of this study we reviewed cases that did not involve solely implant-based breast reconstruction; it is commonly known that a large number of cases of pLD breast reconstruction involve implant placement, and that in a certain number of cases implant placement may occur following other autologous reconstructive cases (e.g. PTRAM, DIEP).

\section{Cohort selection}

Our cohort comprised all female patients diagnosed with breast cancer (ICD-9 diagnosis codes 174.0, 174.1, 174.2, 174.3, 174.4, 174.5, 174.6 and 174.8) or at increased risk of breast cancer (ICD-9 diagnosis codes 233.0, v16.3, v10.3, v84.01) undergoing unilateral or bilateral total, subcutaneous, simple, extended simple, radical and extended radical mastectomy (ICD-9 procedure codes unilateral mastectomy: 85.34, 85.41, 85.43, 85.45, 85.47, 85.33 and bilateral mastectomy: 85.36, 85.42, 85.44, 85.46, 85.48, 85.35) (Table 1).

Table 1. Inclusion criteria: International Classification of Diseases, Ninth Revision (ICD-9) Diagnosis codes.

\begin{tabular}{lll}
\hline Inclusion criteria & ICD-9 code & Diagnosis \\
\hline Breast cancer & 174.0 & Malignant neoplasm of female breast: nipple and areola \\
174.1 & Malignant neoplasm of female breast: central portion \\
174.2 & Malignant neoplasm of female breast: upper inner-quadrant \\
174.3 & Malignant neoplasm of female breast: lower-inner quadrant \\
174.4 & Malignant neoplasm of female breast: upper-outer quadrant \\
174.5 & Malignant neoplasm of female breast: lower-outer quadrant \\
174.6 & Malignant neoplasm of female breast: axillary tail \\
174.8 & Malignant neoplasm of female breast: other sites \\
174.9 & Malignant neoplasm of female breast: unspecified \\
233.0 & Carcinoma in situ of breast \\
Increased breast cancer risk & & \\
v16.3 & Family history of malignant neoplasm \\
& v84.01 & Personal history of breast malignant neoplasm \\
\hline
\end{tabular}


Patients with multiple ICD-9 procedure code 85.40 ("mastectomy") and v50.41 ("prophylactic removal of the breast") were classified as having bilateral mastectomy (table 1). Implant-based breast reconstructions were defined as any type of mastectomy with concurrent, immediate tissue expander followed by implant insertion at a later date, or as an immediate implant placement (ICD-9 procedure codes 85.33, $85.35,85.53,85.54,85.95,85.96)$ (Table 2).

Table 2. Inclusion criteria: International Classification of Diseases, Ninth Revision (ICD-9) Procedure codes.

\begin{tabular}{|c|c|c|}
\hline Inclusion criteria & ICD-9 code & Description \\
\hline \multicolumn{3}{|l|}{ Mastectomy } \\
\hline & 85.40 & Mastectomy \\
\hline & v50.41 & Prophylactic removal of breast \\
\hline \multicolumn{3}{|c|}{ Unilateral Mastectomy } \\
\hline & 85.34 & Other unilateral subcutaneous mammectomy \\
\hline & 85.41 & Unilateral simple mastectomy \\
\hline & 85.43 & Unilateral extended simple mastectomy \\
\hline & 85.45 & Unilateral radical mastectomy \\
\hline & 85.47 & Unilateral extended radical mastectomy \\
\hline & 85.33 & $\begin{array}{l}\text { Unilateral subcutaneous mammectomy with synchronous } \\
\text { implant }\end{array}$ \\
\hline \multicolumn{3}{|c|}{ Bilateral Mastectomy } \\
\hline & 85.36 & Other bilateral subcutaneous mammectomy \\
\hline & 85.42 & Bilateral simple mastectomy \\
\hline & 85.44 & Bilateral extended simple mastectomy \\
\hline & 85.46 & Bilateral radical mastectomy \\
\hline & 85.48 & Bilateral extended radical mastectomy \\
\hline & 85.35 & $\begin{array}{l}\text { Bilateral subcutaneous mammectomy with synchronous } \\
\text { implant }\end{array}$ \\
\hline \multicolumn{3}{|c|}{ Breast reconstruction } \\
\hline \multicolumn{3}{|c|}{ Tissue Expander/Implant BR } \\
\hline & 85.33 & $\begin{array}{l}\text { Unilateral subcutaneous mammectomy with synchronous } \\
\text { implant }\end{array}$ \\
\hline & 85.35 & $\begin{array}{l}\text { Bilateral subcutaneous mammectomy with synchronous } \\
\text { implant }\end{array}$ \\
\hline & 85.53 & Unilateral breast implant \\
\hline & 85.54 & Bilateral breast implant \\
\hline & 85.95 & Insertion breast tissue expander \\
\hline & 85.96 & Removal breast tissue expander \\
\hline \multicolumn{3}{|l|}{ Autologous BR } \\
\hline & 85.71 & Latissimus dorsi myocutaneous flap (LD), pedicled \\
\hline & 85.72 & $\begin{array}{l}\text { Transverse rectus abdominis myocutaneous (TRAM) flap, } \\
\text { pedicled }\end{array}$ \\
\hline & 85.73 & Transverse rectus abdominis myocutaneous (TRAM) flap, free \\
\hline & 85.74 & Deep inferior epigastric artery perforator (DIEP) flap, free \\
\hline & 85.75 & Superficial inferior epigastric artery (SIEA) flap, free \\
\hline & 85.76 & Gluteal artery perforator (GAP) flap, free \\
\hline
\end{tabular}


Autologous breast reconstructions were divided into individual flap types; LD, pTRAM, fTRAM, DIEP, SIEA and GAP flap (ICD-9 procedure codes 85.71, 85.72, 85.73, 85.74, 85.75 and 85.76, respectively) (Table 2). Due to limited sample size, SIEA and GAP flaps were combined into a "other" flaps category. Male patients, those under 18 years of age, those of unknown gender or age, and those who underwent reconstruction using combined methods (autologous plus implant) were excluded. Furthermore, cases of breast reconstruction without a concurrent mastectomy were considered delayed breast reconstruction and therefore excluded from analysis (Figure 1).

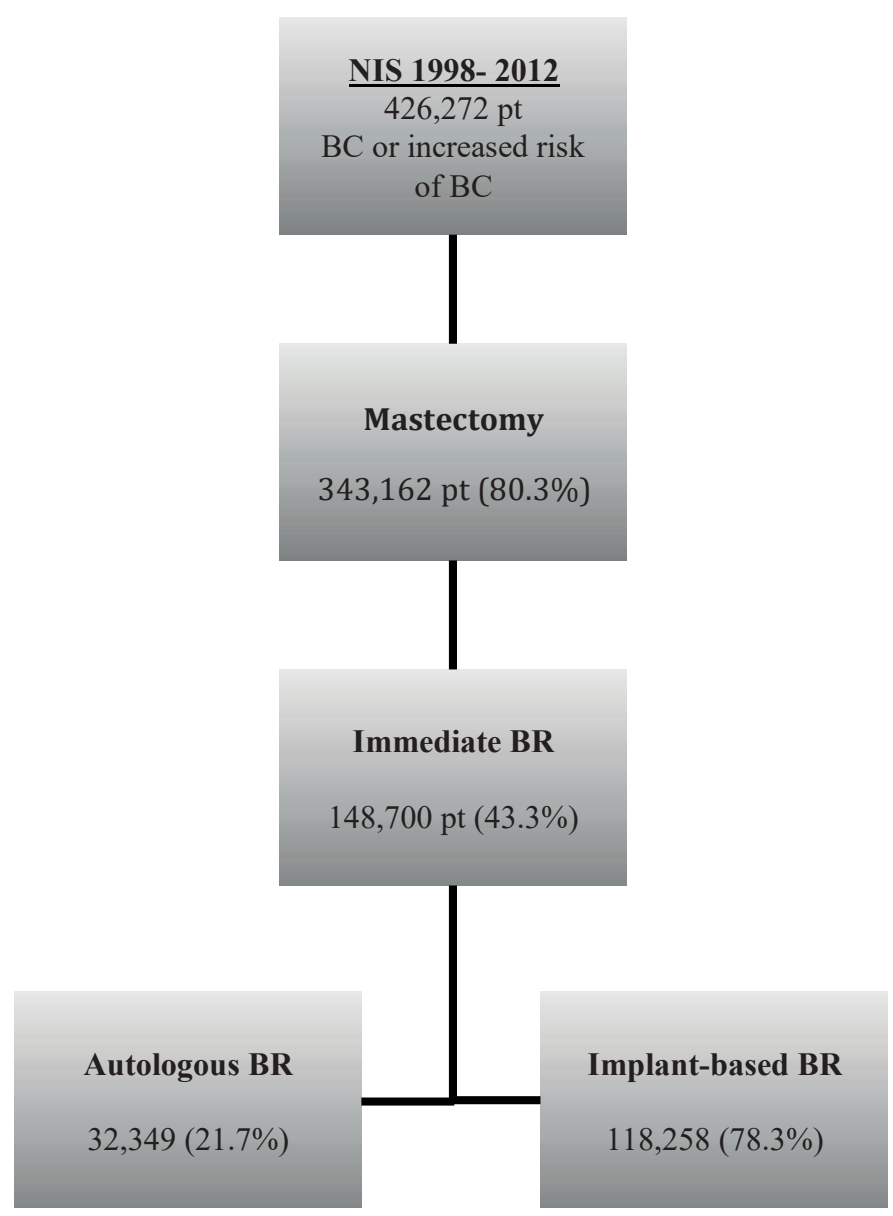

Figure 1. Flowchart inclusion criteria. 


\section{Analyzed variables}

The analyzed data included age, calendar year, primary payer (Medicare, Medicaid, private insurance and other insurance types), geographical region, race, hospital status (teaching/non-teaching, urban/rural), and hospital bedsize. Patients' ages were divided into groups $(<39,40-49,50-59,<60)$. The hospital's census region was obtained from the AHA Annual Survey of Hospitals and is defined by the U.S. Census Bureau. The states included in each region are as follows: Northeast region: $M E, N H, V T, M A, R I, C T, N Y N J$, and PA. Midwest region: $O H, I N, I L, M I, W I, M N, I A, M O, N D, S D, N E$, and KS. South region: $D E, M D, D C, V A, W V, N C, S C, G A, F L, K Y, T N, A L, M S, A R, L A, O K$, and TX. West region includes: $M T, I D, W Y, C O, N M, A Z, U T, N V, W A, O R, C A, A K$, and HI. Hospital teaching status is determined by the Nationwide Inpatients Sample as those hospitals with American Medical Association-approved residency programs, or are members of the council of Teaching Hospitals, or have a ratio of full-time residents to beds of 0.25 or greater. ${ }^{22}$ In the NIS, race is classified into similar categories as census population data (White, Black, Hispanic, Asian or Pacific Islander, Native American and other). Due to limited sample sizes, Native Americans were combined into the "other" category for analysis. Hospital bed size categories were determined by the Nationwide Inpatient Sample based on hospital beds and are specific to the hospital's location and teaching status. Small bedsize was determined as 1-49 for a rural hospital, 1-99 for an urban nonteaching hospital and 1-299 for an urban, teaching hospital. Medium bedsize was determined as 50-99 for a rural hospital, 100-199 for an urban non-teaching hospital and 300-499 beds for an urban teaching hospital. Large bedsize was determined as $100+$ for a rural hospital, 200+ for an urban non-teaching hospital and 500+ for an urban teaching hospital. ${ }^{22}$ Estimated household income, as determined by median household income for the patient's zip code, was recorded directly from the NIS database and stratified by quartile, indicating the poorest to wealthiest populations.

\section{Statistical analysis}

In order to facilitate trend analysis using multiple years of NIS data, the discharge weight factor provided by the NIS was utilized, and national estimates were calculated by uniformly applying the weight factor to the discharges. ${ }^{23}$ Descriptive statistics were reported as proportions and compared using Pearson's Chi-square. Trends were analyzed using the Cochran-Armitage test for trend. All statistical analyses were performed using IBM SPSS version 22.0 (IBM Corp., Armonk, NY) and significance was set at $p<0.05$. 


\section{RESULTS}

\section{Autologous Breast Reconstruction}

Our cohort comprised 427,272 patients diagnosed with breast cancer or at increased risk of breast cancer. A total of 343,163 (80.3\%) underwent mastectomy and of these, 148,700 (43.3\%) underwent immediate breast reconstruction (tissue expander/implant or autologous BR). Within the breast reconstruction group, 32,249 patients $(21.7 \%)$ underwent autologous BR and 118,258 patients (78.3\%) implant-based BR (Figure 1).

A detailed distribution of the autologous breast reconstruction group is presented in Table 3. Median age was $51.6 \pm 9.6$ years. The majority of women undergoing autologous breast reconstruction were Caucasian $(65.2 \% ; n=21,016)$, aged $50-59$ years (35.7\%; $n=11,504)$, undergoing unilateral reconstruction (55.6\%; $n=17,939)$, privately insured $(79.4 \% ; n=25,607)$ and with an estimated household income in the $4^{\text {th }}$ quartile (41.4\%; $n=13,028)$. Furthermore, a vast majority of autologous breast reconstructions were performed in urban $(98.6 \% ; n=31,570)$, teaching hospitals $(79.9 \% ; n=24,601)$ with large bedsize (71.9\%; $n=22,999)$. Overall, most autologous breast reconstructions were performed in the Southern region (37.4\%; $n=12,052)$ followed by the Northeast $(28.1 \%$; $n=9050)$, Midwest $(18.4 \%, n=5,919)$ and the West region $(16.2 \% ; n=5,226)$. (Table 3, Figure 2a-b).

When stratified per region, autologous BR demonstrated a significant increase over time in the Southern region (from 35.6\% to 43.4\% between 2008 to 2011, respectively, followed by a decrease to $38.9 \%$ in $2012, p<0.001$ test for trend). In the West region, after an initial increase from $16.0 \%$ to $19.3 \%$ between 2008 and 2010, a decrease to $14.6 \%$ in 2012 ( $p<0.001$ test for trend) was observed. The Midwest region showed a stable increase and decrease over the years (from 14.8\% to 20.8\% between 2008-2009, $15.3 \%$ in $2010,20.0 \%$ in 2011 to $17.7 \%$ in 2012, $p<0.001$ test for trend), and finally, autologous BR decreased over time in the Northeast region (from 33.5\% in 2008 to $28.9 \%$ in 2012, $p<0.001$ test for trend) (Figure 3). 
Table 3. Detailed distribution of patients undergoing Autologous Breast Reconstruction.

\begin{tabular}{|c|c|c|}
\hline Characteristics & Total n & $\%$ \\
\hline Median age +/-SD, yr & $51.6+/-9.6$ & \\
\hline \multicolumn{3}{|l|}{ Age } \\
\hline$<39 y$ & 3104 & $9.6 \%$ \\
\hline $40-49 y$ & 10427 & $32.3 \%$ \\
\hline $50-59 y$ & 11504 & $35.7 \%$ \\
\hline$>60 y$ & 7213 & $22.4 \%$ \\
\hline \multicolumn{3}{|l|}{ Race } \\
\hline White & 21016 & $65.2 \%$ \\
\hline Black & 3895 & $12.1 \%$ \\
\hline Hispanic & 2563 & $7.9 \%$ \\
\hline Asian & 1005 & $3.1 \%$ \\
\hline Other & 3770 & $11.7 \%$ \\
\hline \multicolumn{3}{|l|}{ Type of mastectomy } \\
\hline Unilateral & 17939 & $55.6 \%$ \\
\hline Bilateral & 14310 & $44.4 \%$ \\
\hline \multicolumn{3}{|c|}{ Estimated household income } \\
\hline 4th quartile & 13028 & $41.1 \%$ \\
\hline 3rd quartile & 8307 & $26.2 \%$ \\
\hline 2nd quartile & 5687 & $17.9 \%$ \\
\hline 1st quartile & 4508 & $14.2 \%$ \\
\hline \multicolumn{3}{|l|}{ Teaching status } \\
\hline non-teaching & 7406 & $23.1 \%$ \\
\hline teaching & 24601 & $76.9 \%$ \\
\hline \multicolumn{3}{|l|}{ Hospital Bedsize } \\
\hline small & 3607 & $11.3 \%$ \\
\hline medium & 5401 & $16.9 \%$ \\
\hline large & 22999 & $71.9 \%$ \\
\hline \multicolumn{3}{|l|}{ Hospital Location } \\
\hline Rural & 437 & $1.4 \%$ \\
\hline Urban & 31570 & $98.6 \%$ \\
\hline \multicolumn{3}{|l|}{ Insurance type } \\
\hline Private & 25607 & $79.4 \%$ \\
\hline Medicare & 3420 & $10.6 \%$ \\
\hline Medicaid & 1976 & $6.1 \%$ \\
\hline Other & 1246 & $3.9 \%$ \\
\hline \multicolumn{3}{|l|}{ Region } \\
\hline Northeast & 9050 & $28.1 \%$ \\
\hline Midwest & 5919 & $18.4 \%$ \\
\hline South & 12052 & $37.4 \%$ \\
\hline West & 5226 & $16.2 \%$ \\
\hline
\end{tabular}




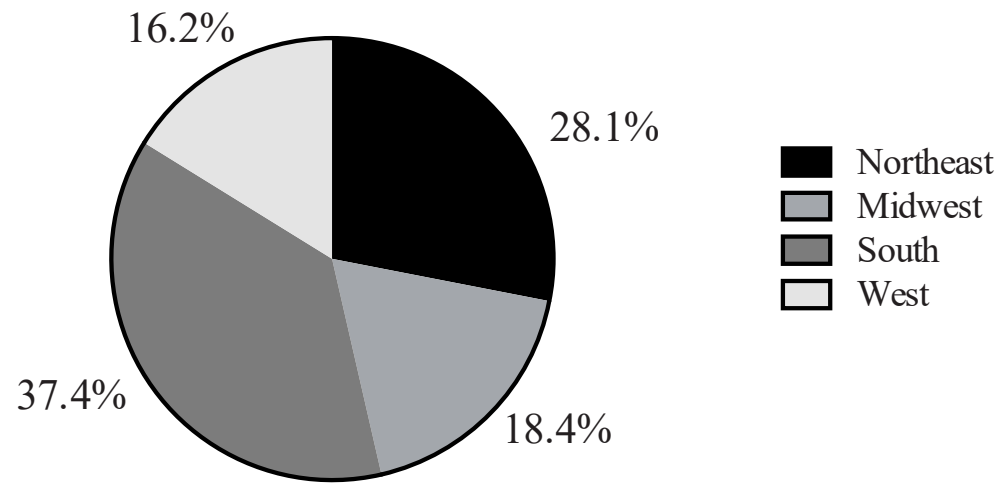

Total $=32249$

Figure 2a: Percentage Autologous Breast Reconstruction stratified per region.

\section{Autologous Breast Reconstruction Procedures}

Stratified by Region: 2008-2012

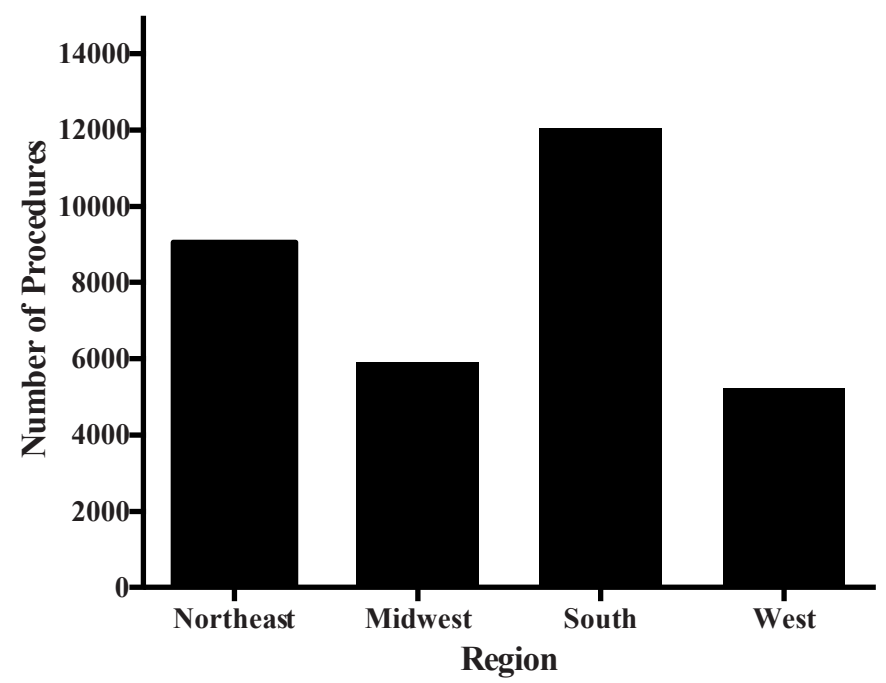

Figure 2b. Total number of Autologous Breast Reconstruction stratified per region.

\section{Autologous Breast Reconstruction Stratified by Flap}

Subgroup analysis of autologous flap type was performed and is presented in Table 4. The most prevalent type of autologous BR performed was the DIEP flap $(32.4 \%$; $n=10,436)$ followed by the LD flap $(25.8 \% ; n=8,317)$, pTRAM $(20.4 \% ; n=6,577)$, fTRAM $(19.0 \% ; n=6,115)$ and other flaps $(2.5 \% ; n=805)(p<0.001)$ (Table 4). Among all flap 
types, a majority of women were Caucasian, privately insured and operated on in an urban teaching hospital with a large bedsize (Table 4). Interestingly, in DIEP flap BR, a majority of women were aged $40-49$ years and underwent bilateral reconstruction, while in LD, pTRAM, fTRAM and other flap types, the majority of women were aged 50 59 years and underwent unilateral reconstruction (Table 4). Ultimately, most LD (41.0\%; $n=3,408)$ and DIEP flaps $(43.8 \% ; n=4,571)$ were performed in the Southern region, while most pTRAM (35.7\%; $n=2,350)$, fTRAM (32.9\%; $n=2,013)$ and other flaps (35.0\%; $n=281$ ) flaps were performed in the Northeast region (Table 4).

\section{Autologous Breast Reconstruction Procedures Stratified by Region: 2008-2012}

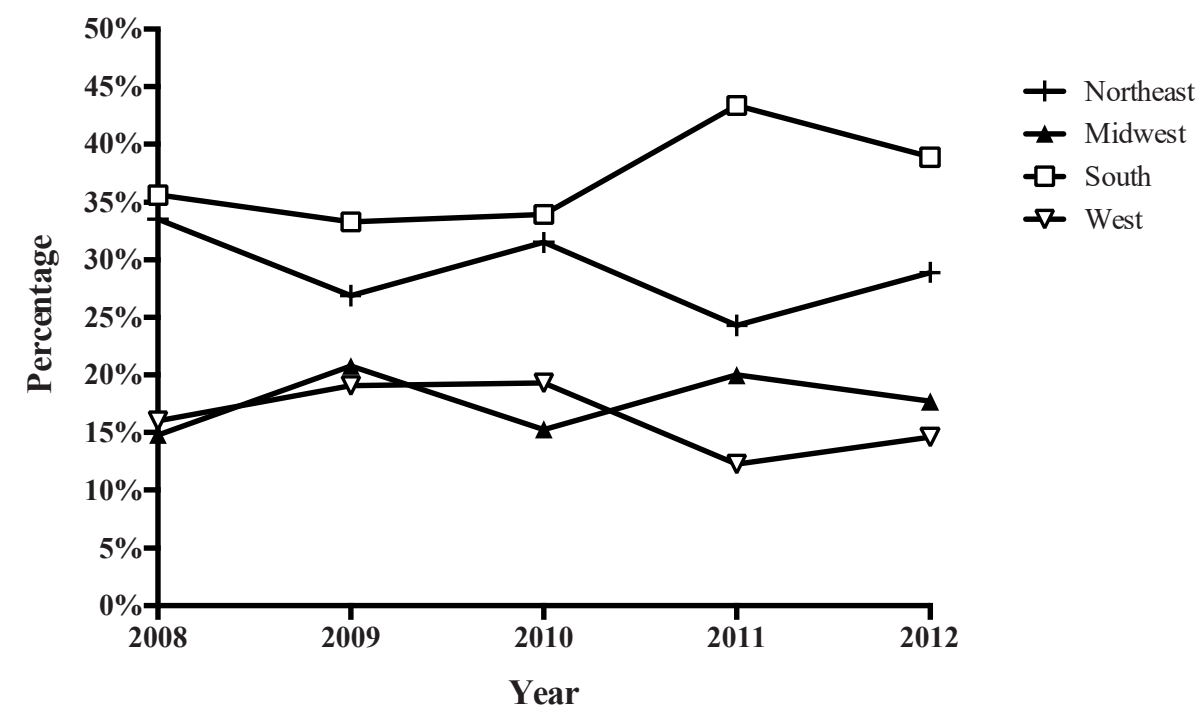

Figure 3. Regional trend over time Autologous Breast Reconstruction. 
Chapter 3

Table 4. Detailed distribution of patients undergoing Autologous Breast Reconstruction stratified per flap.

\begin{tabular}{|c|c|c|c|c|c|c|c|c|c|c|}
\hline \multirow[b]{2}{*}{ Characteristics } & \multicolumn{2}{|l|}{ LD } & \multicolumn{2}{|l|}{ PTRAM } & \multicolumn{2}{|l|}{ fTRAM } & \multicolumn{2}{|l|}{ DIEP } & \multicolumn{2}{|l|}{ Other } \\
\hline & $(n=)$ & (\%) row & $(n=)$ & (\%) row & $(n=)$ & (\%) row & $(n=)$ & (\%) row & $(n=)$ & (\%) row \\
\hline Total & 8,317 & $25.8 \%$ & 6,577 & $20.4 \%$ & 6,115 & $19.0 \%$ & 10,436 & $32.4 \%$ & 802 & $2.5 \%$ \\
\hline \multicolumn{11}{|l|}{ Age* } \\
\hline$<39 y$ & 876 & $10.5 \%$ & 494 & $7.5 \%$ & 567 & $9.3 \%$ & 1,110 & $10.6 \%$ & 57 & $7.1 \%$ \\
\hline $40-49 y$ & 2,200 & $26.5 \%$ & 2,019 & $30.7 \%$ & 2,115 & $34.6 \%$ & 3,815 & $36.6 \%$ & 279 & $34.8 \%$ \\
\hline $50-59 y$ & 2,817 & $33.9 \%$ & 2,436 & $37.0 \%$ & 2,163 & $35.4 \%$ & 3,750 & $35.9 \%$ & 337 & $42.0 \%$ \\
\hline$>60 y$ & 2,424 & $29.1 \%$ & 1,629 & $24.8 \%$ & 1,271 & $20.8 \%$ & 1,761 & $16.9 \%$ & 129 & $16.1 \%$ \\
\hline \multicolumn{11}{|l|}{ Race* } \\
\hline White & 5,537 & $66.6 \%$ & 4,489 & $68.3 \%$ & 3,746 & $61.2 \%$ & 6,662 & $63.8 \%$ & 583 & $72.6 \%$ \\
\hline Black & 1,026 & $12.3 \%$ & 871 & $13.2 \%$ & 752 & $12.3 \%$ & 1,209 & $11.6 \%$ & 37 & $4.6 \%$ \\
\hline Hispanic & 452 & $5.4 \%$ & 448 & $6.8 \%$ & 510 & $8.3 \%$ & 1,104 & $10.6 \%$ & 48 & $6.0 \%$ \\
\hline Asian & 195 & $2.3 \%$ & 171 & $2.6 \%$ & 198 & $3.2 \%$ & 408 & $3.9 \%$ & 34 & $4.2 \%$ \\
\hline Other & 1,107 & $13.3 \%$ & 598 & $9.1 \%$ & 910 & $14.9 \%$ & 1,053 & $10.1 \%$ & 101 & $12.6 \%$ \\
\hline \multicolumn{11}{|c|}{ Type of mastectomy* } \\
\hline Unilateral & 5,009 & $60.2 \%$ & 4,535 & $69.0 \%$ & 3,661 & $59.9 \%$ & 4,298 & $41.2 \%$ & 435 & $54.2 \%$ \\
\hline Bilateral & 3,308 & $39.8 \%$ & 2,042 & $31.0 \%$ & 2,454 & $40.1 \%$ & 6,138 & $58.8 \%$ & 367 & $45.8 \%$ \\
\hline \multicolumn{11}{|c|}{ Estimated household income* } \\
\hline 4th quartile & 3,097 & $37.7 \%$ & 2,362 & $36.6 \%$ & 2,448 & $41.1 \%$ & 4,732 & $45.9 \%$ & 389 & $50.3 \%$ \\
\hline 3rd quartile & 2,265 & $27.5 \%$ & 1,788 & $27.7 \%$ & 1,622 & $27.3 \%$ & 2,448 & $23.7 \%$ & 183 & $23.7 \%$ \\
\hline 2nd quartile & 1,592 & $19.4 \%$ & 1,370 & $21.2 \%$ & 1,030 & $17.3 \%$ & 1,580 & $15.3 \%$ & 115 & $14.9 \%$ \\
\hline 1st quartile & 1,197 & $14.6 \%$ & 891 & $13.8 \%$ & 823 & $13.8 \%$ & 1,515 & $14.7 \%$ & 81 & $10.5 \%$ \\
\hline \multicolumn{11}{|l|}{ Teaching status* } \\
\hline non-teaching & 2,449 & $29.8 \%$ & 2,020 & $31.0 \%$ & 1,351 & $22.2 \%$ & 1,467 & $14.1 \%$ & 119 & $15.1 \%$ \\
\hline teaching & 5,782 & $70.2 \%$ & 4,500 & $69.0 \%$ & 4,732 & $77.8 \%$ & 8,918 & $85.9 \%$ & 669 & $84.9 \%$ \\
\hline \multicolumn{11}{|c|}{ Hospital Bedsize* } \\
\hline small & 1,244 & $15.1 \%$ & 816 & $12.5 \%$ & 662 & $10.9 \%$ & 817 & $7.9 \%$ & 69 & $8.7 \%$ \\
\hline medium & 1,766 & $21.5 \%$ & 1,325 & $20.3 \%$ & 971 & $16.0 \%$ & 1,216 & $11.7 \%$ & 123 & $15.6 \%$ \\
\hline large & 5,220 & $63.4 \%$ & 4,379 & $67.2 \%$ & 4,449 & $73.2 \%$ & 8,353 & $80.4 \%$ & 597 & $75.7 \%$ \\
\hline \multicolumn{11}{|c|}{ Hospital Location* } \\
\hline Rural & 136 & $1.7 \%$ & 170 & $2.6 \%$ & 83 & $1.4 \%$ & 27 & $0.3 \%$ & 22 & $2.8 \%$ \\
\hline Urban & 8,094 & $98.3 \%$ & 6,351 & $97.4 \%$ & 6,000 & $98.6 \%$ & 10,359 & $99.7 \%$ & 767 & $97.2 \%$ \\
\hline \multicolumn{11}{|l|}{ Insurance type* } \\
\hline Private & 6,102 & $73.4 \%$ & 5,134 & $78.0 \%$ & 4,708 & $77.0 \%$ & 8,965 & $85.9 \%$ & 698 & $86.9 \%$ \\
\hline Medicare & 1,364 & $16.4 \%$ & 733 & $11.1 \%$ & 648 & $10.6 \%$ & 633 & $6.1 \%$ & 42 & $5.2 \%$ \\
\hline Medicaid & 587 & $7.1 \%$ & 423 & $6.4 \%$ & 472 & $7.7 \%$ & 460 & $4.4 \%$ & 34 & $4.2 \%$ \\
\hline Other & 265 & $3.2 \%$ & 288 & $4.4 \%$ & 287 & $4.7 \%$ & 378 & $3.6 \%$ & 29 & $3.6 \%$ \\
\hline \multicolumn{11}{|l|}{ Region* } \\
\hline Northeast & 1,705 & $20.5 \%$ & 2,350 & $35.7 \%$ & 2,013 & $32.9 \%$ & 2,701 & $25.9 \%$ & 281 & $35.0 \%$ \\
\hline Midwest & 1,831 & $22.0 \%$ & 1,195 & $18.2 \%$ & 1,360 & $22.2 \%$ & 1,374 & $13.2 \%$ & 160 & $20.0 \%$ \\
\hline South & 3,408 & $41.0 \%$ & 2,269 & $34.5 \%$ & 1,601 & $26.2 \%$ & 4,571 & $43.8 \%$ & 204 & $25.4 \%$ \\
\hline West & 1,372 & $16.5 \%$ & 764 & $11.6 \%$ & 1,142 & $18.7 \%$ & 1,791 & $17.2 \%$ & 157 & $19.6 \%$ \\
\hline
\end{tabular}

$* p<0.001$ 
Trend analysis revealed a nationwide significant increase in LD and DIEP flap from 24.5\% to $28.6 \%$ ( $p<0.001$ test for trend) and from $19.5 \%$ to $35.4 \%$ ( $p<0.001$ test for trend) between 2008 and 2012, respectively). A decrease in the incidence and overall percentage was seen in pTRAM (from $26.9 \%$ to $15.6 \%, p<0.001$ test for trend), fTRAM (from $26.7 \%$ to $18.2 \%, p<0.001$ test for trend) and "other" flaps (from $2.3 \%$ to $2.2 \%$ ) over the same time period (Figure 4). When stratified per region, DIEP and the LD flap demonstrated a significant increase over time in all regions (Figure $5 a-d)(p<0.001)$, and PTRAM and fTRAM flap decreased significantly in all regions over the same time period $(p<0.001)$ (Figure 6a-d). "Other" flap types increased in the Southern and Western regions $(p<0.001)$, and decreased in the Northeastern and Midwestern regions $(p<0.001)$ (figure 5a-d).

Autologous Breast ReconstrucOons: 2008 - 2012

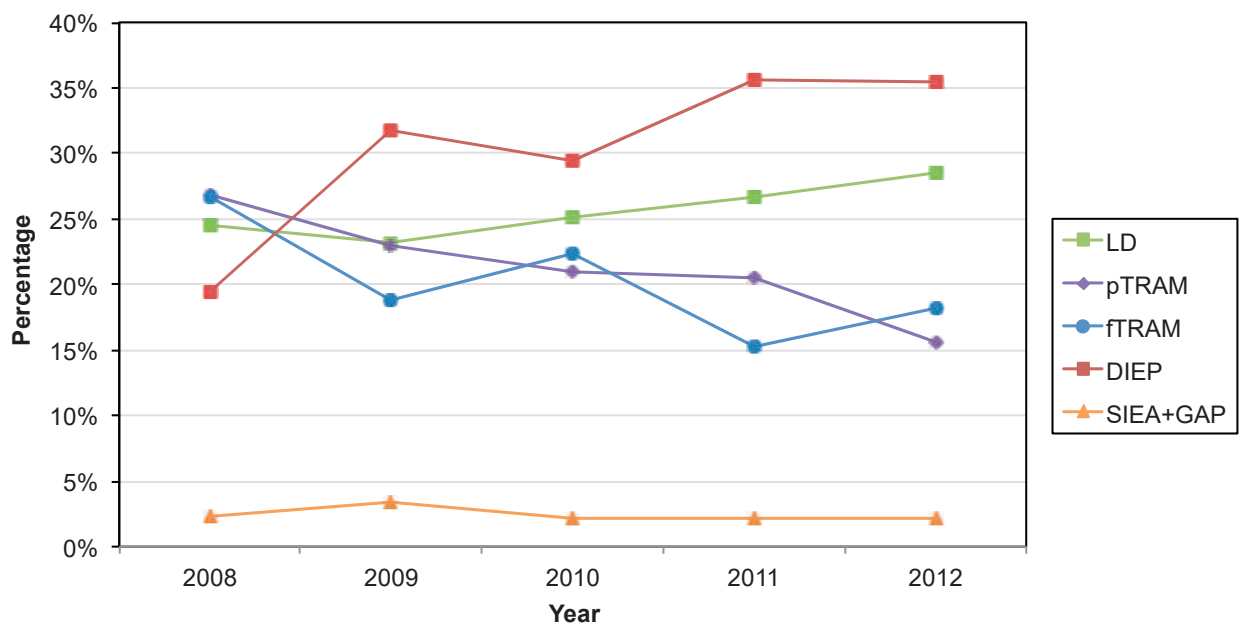

Figure 4. National trend over time in Autologous Breast Reconstruction stratified per flap. 


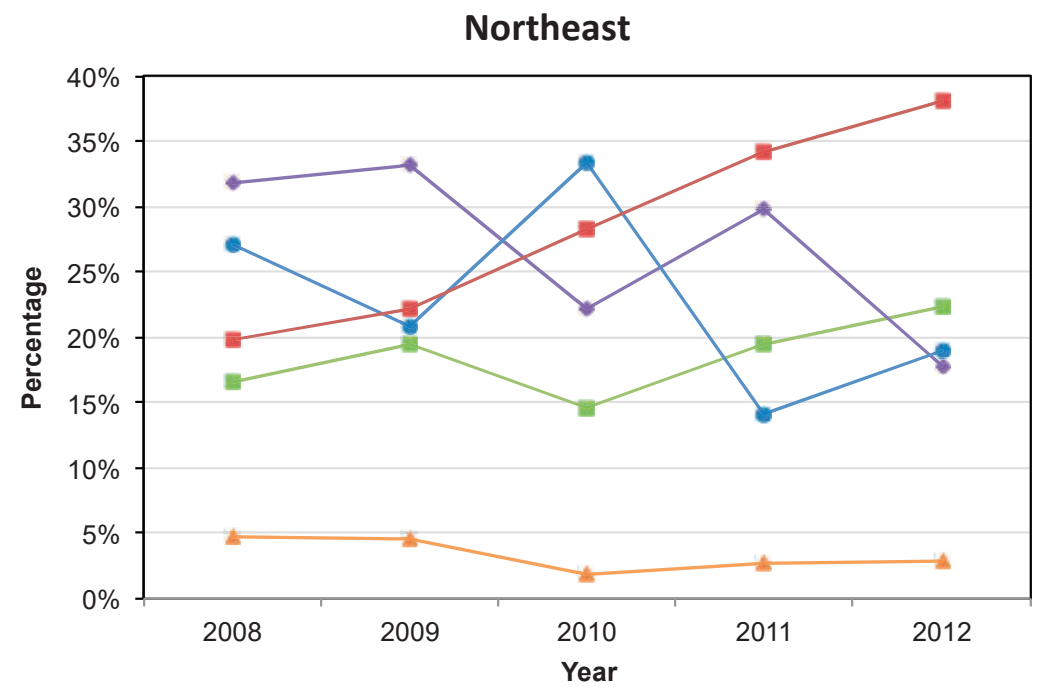

Figure 5a. Regional trend over time in Autologous Breast Reconstruction stratified per flap. Northeast region

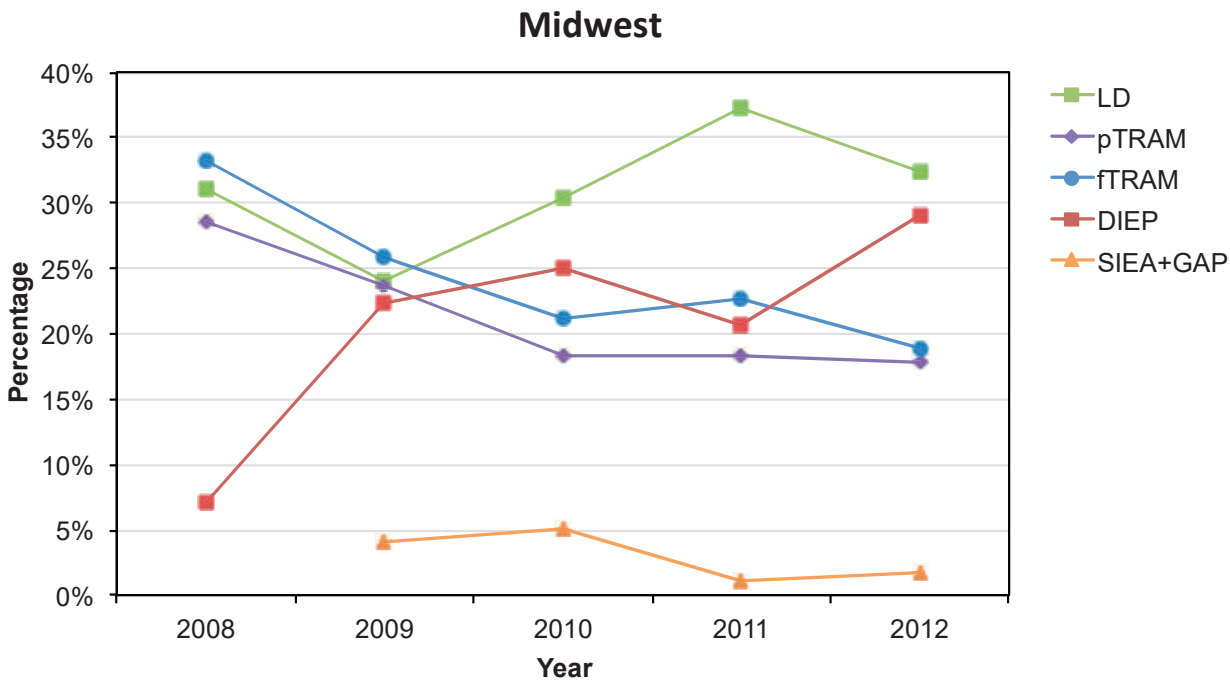

Figure 5b. Regional trend over time in Autologous Breast Reconstruction stratified per flap. Midwest region 


\section{South}

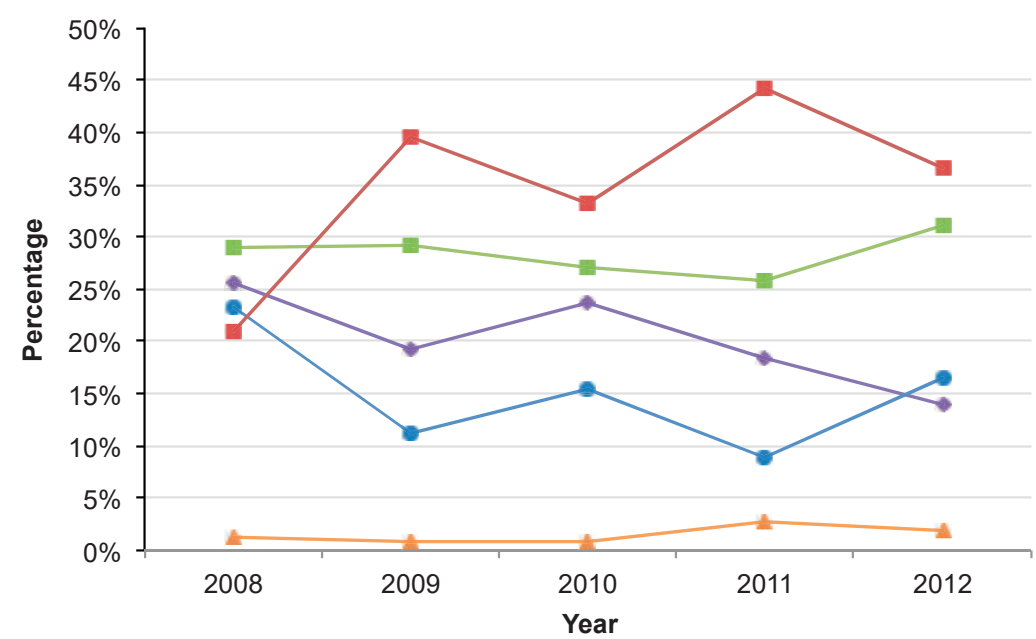

Figure 5c. Regional trend over time in Autologous Breast Reconstruction stratified per flap. South region

West

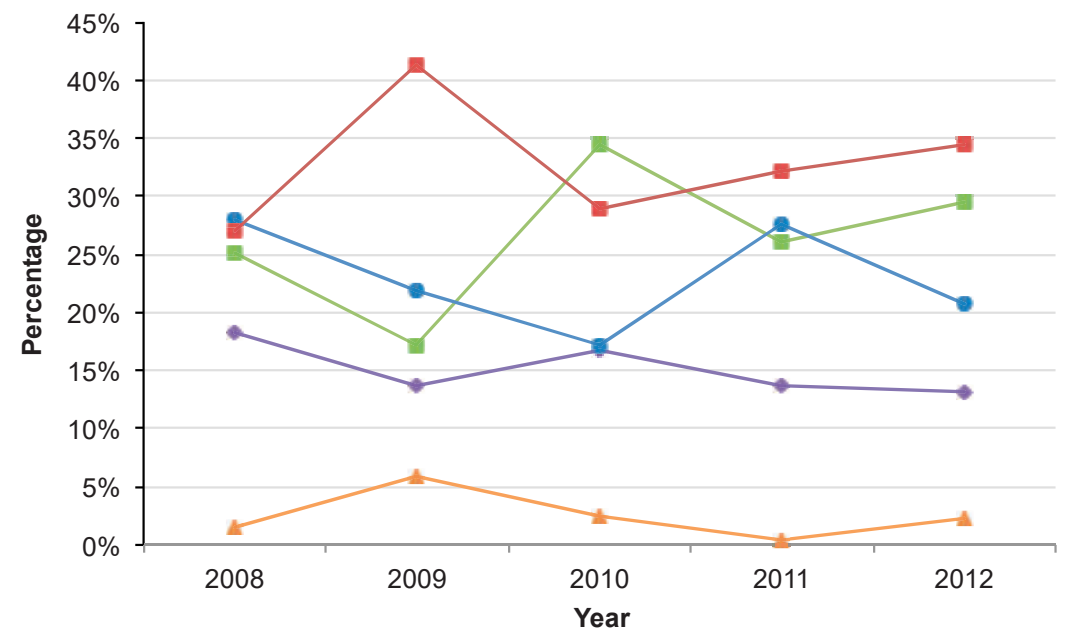

Figure $5 \mathrm{~d}$. Regional trend over time in Autologous Breast Reconstruction stratified per flap. West region 


\section{DISCUSSION}

The NIS database is a valuable tool for analyzing and describing breast reconstructive trends. In this study, we analyzed breast reconstruction trends for autologous flaps performed in the United States from 2008 to 2012 using the National Inpatient Sample Database (NIS-HCUP), and found a stable increase over time in DIEP and LD flaps, and a significant decrease in PTRAM, fTRAM and other flap types over the same time period. Overall, most autologous breast reconstructive procedures were performed in the Southern region, which was also the only region showing a significant increase over time in the total autologous breast reconstructions performed (Figure 3).

A literature review of studies prior to 2008 reveal a consistent trend with regards to reconstruction choice. While implant based breast reconstruction rates were found to be steady, Manahan et al. ${ }^{24}$ described decreased rates of TRAM procedures and an increase in the use of DIEP flap reconstruction over time. Although a similar trend was noted in our study, the total number of autologous reconstructions did not increase. In fact, analysis of the various breast reconstruction types from 1998 to 2007 using the MarketScan database demonstrated an overall decrease in the number of free flap breast reconstructions performed. Our observation is consistent with Pien et al. ${ }^{16}$ who found that the total amount of autologous breast reconstruction discharges was relatively stable from 2009-2011. In spite of the advantages of expander/implant-based reconstructions, presented by Albornoz et al. ${ }^{10}$, it is evident that implant based breast reconstruction remains a more popular choice.

In all autologous flap reconstructions, 97.2\%-99.7\% were performed in urban, and 69.0\%-84.9\% were performed in a teaching hospital (Table 4). Masoomi et al. ${ }^{25}$ presented similar observations in 2014, where three-fourths of autologous reconstructions were performed at teaching hospitals. They reported that in spite of more complex free flap breast reconstructions being performed at teaching hospitals, the final clinical outcomes for both breast reconstructions and patient satisfaction were similar between teaching and non-teaching hospitals.

Despite a global preference for the DIEP flap autologous reconstructions, there is still concern about procedural cost in comparison with expander/implant-based reconstruction. In general, microsurgical techniques, especially for bilateral cases, are associated with greater health care costs. DIEP flap reconstruction in particular is associated with higher charges and costs compared to PLD, PTRAM, and fTRAM flaps. However, due to a lower rate of unplanned surgical revisions, long-term cost in the autologous group remains less. Furthermore, DIEP flap is the current gold standard for microsurgical breast reconstruction, and its popularity among other autologous flaps is increasing each year. This notion is corroborated in our results. In addition, although implant reconstruction remains less expensive over an average of three years, patients will often require more revisions. ${ }^{26,27}$ Hence, in the long run, this small financial benefit may disappear over time. 
Traditionally, patients undergoing radiation therapy prior to breast reconstruction are not considered ideal candidates for implant-only breast reconstruction due to concerns for adverse tissue changes. A recent trend analysis using the Surveillance, Epidemiology, and End Results (SEER) database published by Agarwal et al. demonstrated decreased free flap reconstruction rates in previously irradiated breasts. 28,29 This finding is consistent with previously published studies using the SEER database, suggesting that these days, surgeons may become increasingly willing to perform implant based reconstruction in radiated patients. Despite the numerous advantages that expander/implant-based reconstructions provide, not all patients qualify for this type of repair. However, no definitive guidelines exist for the reconstruction selection process with procedure choice based primarily on surgeon judgment and patients' preference.

Despite more natural outcomes and fewer procedures performed per patient, the learning curve for autologous breast reconstruction is longer. However, as the number of surgeons trained in microsurgical procedures has increased it has become standard of care at numerous institutions. Greater accessibility to microsurgical training may contribute to the future rise in microsurgical reconstructions.

The study is not exempt from limitations. The National Inpatient Sample relies on the administrative data. On many occasions, it is impossible to include all the patients due to an imperfect coding system (related to over or under-coding of procedures or diagnoses, novel ICD-9 coding and registration for flaps from 2008), the retrospective nature of the data, and missing data. These factors could lead to over or under analysis. In our study, we utilized a corrective algorithm to produce the most coherent and reliable study outcome.

\section{CONCLUSION}

Overall, most autologous breast reconstructive procedures were performed in the Southern region, which was also the only region showing a significant increase over time in the total autologous breast reconstructions performed $(p<0.001)$. DIEP and LD flaps increased significantly over time, both nationally and regionally. Additionally, we found a significant decrease in PTRAM, fTRAM and other flap types over the same time period. 


\section{TABLE AND FIGURE LEGEND}

Table 1. Inclusion criteria: International Classification of Diseases, Ninth Revision (ICD-9) Diagnosis codes.

Table 2. Inclusion criteria: International Classification of Diseases, Ninth Revision (ICD-9) Procedure codes.

Table 3. Detailed distribution of patients undergoing Autologous Breast Reconstruction. Table 4. Detailed distribution of patients undergoing Autologous Breast Reconstruction stratified per flap. $* p<0.001$

Figure 1. Flowchart inclusion criteria.

Figure 2a. Percentage Autologous Breast Reconstruction stratified per region.

Figure $2 \mathrm{~b}$. Total number of Autologous Breast Reconstruction stratified per region.

Figure 3. Regional trend over time Autologous Breast Reconstruction.

Figure 4. National trend over time in Autologous Breast Reconstruction stratified per flap. ${ }^{*} p<0.001$

Figure 5a-d. Regional trend over time in Autologous Breast Reconstruction stratified per flap.

Figure 5a. Northeast region

Figure 5b. Midwest region

Figure $5 c$. South region

Figure $5 \mathrm{~d}$. West region 


\section{REFERENCES}

1. Erickson B, Hurowitz R, Jeffery $C$, et al. Acoustic rhinometry and video endoscopic scoring to evaluate postoperative outcomes in endonasal spreader graft surgery with septoplasty and turbinoplasty for nasal valve collapse. Journal of otolaryngology - head \& neck surgery = Le Journal d'oto-rhino-laryngologie et de chirurgie cervico-faciale 2016;45:2

2. Kummerow KL, Du L, Penson DF, et al. Nationwide trends in mastectomy for early-stage breast cancer. JAMA surgery 2015;150:9-16

3. Rowland JH, Desmond KA, Meyerowitz BE, et al. Role of breast reconstructive surgery in physical and emotional outcomes among breast cancer survivors. Journal of the National Cancer Institute 2000;92:1422-1429

4. Atisha D, Alderman AK, Lowery JC, et al. Prospective analysis of long-term psychosocial outcomes in breast reconstruction: two-year postoperative results from the Michigan Breast Reconstruction Outcomes Study. Annals of surgery 2008;247:1019-1028

5. Nano MT, Gill PG, Kollias J, et al. Psychological impact and cosmetic outcome of surgical breast cancer strategies. ANZ journal of surgery 2005;75:940-947

6. Dean C, Chetty U, Forrest AP. Effects of immediate breast reconstruction on psychosocial morbidity after mastectomy. Lancet 1983;1:459-462

7. Macadam SA, Ho AL, Lennox PA, et al. Patient-reported satisfaction and health-related quality of life following breast reconstruction: a comparison of shaped cohesive gel and round cohesive gel implant recipients. Plastic and reconstructive surgery 2013;131:431-441

8. Anderson SG, Rodin J, Ariyan S. Treatment considerations in postmastectomy reconstruction: their relative importance and relationship to patient satisfaction. Annals of plastic surgery 1994;33:263-270; discussion 270-261

9. Jagsi R, Jiang J, Momoh AO, et al. Trends and variation in use of breast reconstruction in patients with breast cancer undergoing mastectomy in the United States. Journal of clinical oncology : official journal of the American Society of Clinical Oncology 2014;32:919-926

10. Albornoz CR, Bach PB, Mehrara BJ, et al. A paradigm shift in U.S. Breast reconstruction: increasing implant rates. Plastic and reconstructive surgery 2013;131:15-23

11. Mock V. Body image in women treated for breast cancer. Nursing research 1993;42:153-157

12. Habermann EB, Abbott A, Parsons HM, et al. Are mastectomy rates really increasing in the United States? Journal of clinical oncology : official journal of the American Society of Clinical Oncology 2010;28:3437-3441

13. Tuttle TM, Rueth NM, Abbott A, et al. Trends in the local treatment of breast cancer: should we be worried? Journal of surgical oncology 2011;103:313-316

14. Wilkins EG, Alderman AK. Breast reconstruction practices in north america: current trends and future priorities. Seminars in plastic surgery 2004;18:149-155

15. 2014 Plastic Surgery Procedural Statistics. American Society of Plastic Surgeons. Available at: http://www.plasticsurgery.org/Documents/news-resources/statistics/2014-statistics/plastic-surgerystatsitics-full-report.pdf. Assessed December 1st 2015.

16. Pien I, Caccavale S, Cheung MC, et al. Evolving Trends in Autologous Breast Reconstruction: Is the Deep Inferior Epigastric Artery Perforator Flap Taking Over? Annals of plastic surgery 2014

17. Albornoz CR, Bach PB, Pusic AL, et al. The influence of sociodemographic factors and hospital characteristics on the method of breast reconstruction, including microsurgery: a U.S. population-based study. Plastic and reconstructive surgery 2012;129:1071-1079

18. Albornoz CR, Cordeiro PG, Farias-Eisner G, et al. Diminishing relative contraindications for immediate breast reconstruction. Plastic and reconstructive surgery 2014;134:363e-369e

19. Sun CS, Cantor SB, Reece GP, et al. Helping patients make choices about breast reconstruction: a decision analysis approach. Plastic and reconstructive surgery 2014;134:597-608 
20. U.S. Department of Health \& Human Services. Agency for Healthcare Research and Quality (HCUP). NIS description. Information available at: http://www.hcup-us.ahrq.gov/databases.jsp Assessed: June 15th 2015.

21. Albornoz CR, Cordeiro PG, Mehrara BJ, et al. Economic implications of recent trends in U.S. immediate autologous breast reconstruction. Plastic and reconstructive surgery 2014;133:463-470

22. U.S. Department of Health \& Human Services. Agency for Healthcare Research and Quality (HCUP). NIS description of data elements. Available at: http://www.hcup-us.ahrq.gov/db/vars/h_bedsz/nisnote.jsp Assessed: June 15th 2015.

23. U.S. Department of Health \& Human Services. Agency for Healthcare Research and Quality (HCUP). NIS description of trend weight factor. Available at: http://www.hcup-us.ahrq.gov/db/nation/nis/ trendwghts.jsp Assessed: June 1st, 2015.

24. Manahan MA, Prucz RB, Shridharani SM, et al. Long-term follow-up of changing practice patterns in breast reconstruction due to increased use of tissue expanders and perforator flaps. Microsurgery 2014;34:595-601

25. Masoomi H, Wirth GA, Paydar KZ, et al. Perioperative outcomes of autologous breast reconstruction surgery in teaching versus nonteaching hospitals. Plastic and reconstructive surgery 2014;134:514e-520e

26. Fischer JP, Wes AM, Nelson JA, et al. Propensity-matched, longitudinal outcomes analysis of complications and cost: comparing abdominal free flaps and implant-based breast reconstruction. Journal of the American College of Surgeons 2014;219:303-312

27. Atherton DD, Hills AJ, Moradi $P$, et al. The economic viability of breast reconstruction in the UK: comparison of a single surgeon's experience of implant; LD; TRAM and DIEP based reconstructions in 274 patients. Journal of plastic, reconstructive \& aesthetic surgery : JPRAS 2011;64:710-715

28. Agarwal S, Kidwell KM, Farberg A, et al. Immediate Reconstruction of the Radiated Breast: Recent Trends Contrary to Traditional Standards. Annals of surgical oncology 2015;22:2551-2559

29. Agarwal S, Pappas L, Neumayer L, et al. An analysis of immediate postmastectomy breast reconstruction frequency using the surveillance, epidemiology, and end results database. The breast journal 2011;17:352-358 
Immediate Breast Reconstruction among Patients with Medicare and Private Insurance: A Matched Cohort Analysis

Parisa Kamali Joseph A. Ricci Daniel A. Curiel Justin B. Cohen Anmol Chattha Hinne A. Rakhorst Bernard T. Lee Samuel J. Lin 


\section{ABSTRACT}

\section{Background}

By eliminating economic hurdles, the Women's Health and Cancer Rights Act of 1998 represented a paradigm shift in the availability of breast reconstruction. Yet, many studies report disparities among Medicare insured women. However, these studies do not account for the inherent differences in age and co-morbidities between a younger privately insured and an older Medicare population. We examined immediate breast reconstruction (IBR) utilization between a matched pre- and post-Medicare population.

\section{Methods}

Using the Nationwide Inpatient Sample database (1992 to 2013), breast cancer women undergoing IBR were identified. To minimize confounding medical variables, 64 year-old privately insured women was compared to 66 year-old Medicare insured women. Demographic data, IBR rates, and complication rates were compared. Trend over time was plotted for both cohorts.

\section{Results}

A total of 21,402 sixty-four year-old women and 25,568 sixty-six year-old women were included. Both groups were well matched in terms of demographic type of reconstruction and complication rates. $72.3 \% 64$ year-old and $71.2 \% 66$ year-old women opted for mastectomy. Of these, $25.5 \%(n=3,941) 64$ year-old privately insured and $17.7 \%(n=3,213) 66$ year-old Medicare insured women underwent IBR $(p<0.01)$. During the study period, IBR rates increased significantly in both cohorts in a similar cohort.

\section{Conclusion}

This study demonstrates significant increasing IBR rates in both cohorts. Moreover, after an initial slower upward trend, after a decade IBR in 66 year-old Medicare insured women approaches similar rates of breast reconstruction among those with private insurance. 


\section{INTRODUCTION}

The Women's Health and Cancer Rights Act (WHCRA) was federally enacted in 1998 to ensure all group and individual health plans provide insurance coverage for reconstruction after mastectomy regardless of diagnosis. WHCRA also extended coverage for reconstruction or revision of the contralateral breast in order to produce a symmetrical appearance. This represented a paradigm shift in the availability of reconstruction by eliminating economic hurdles to care.

Although increasing number of women opted for breast reconstruction in the years after the WHCRA, this trend was not consistent across all demographic groups. Significant disparities exist based on access to healthcare, age, race, geographic location, and patient education. ${ }^{1-15}$ As a result, patient education events, such as Breast Reconstruction Awareness Day instituted in 2012, have flourished to increase awareness of breast reconstruction. More recently, the Breast Cancer Patient Education Act, passed in December 2015, was designed to implement an educational campaign to inform women in minority and lower socioeconomic groups about their options for breast reconstruction. ${ }^{16}$

To date, there is a large body of available literature to suggest that insurance status plays a role key in patients' access to immediate breast reconstruction (IBR). 1, 2, 5-7, 9, 17-19 These reports suggest that women with Medicare are significantly less likely to undergo breast reconstruction when compared to women with private insurance. However, many of these studies do not always take the inherent differences in age and comorbidities between a younger privately insured population and an older Medicare population into account. ${ }^{2}$ 4, 5, 8, 20-22 This study aims to bridge this knowledge gap, by performing a matched cohort analysis to examine the differences between pre- and post-Medicare and utilization of breast reconstruction in a matched cohort.

\section{METHODS}

Patient data was obtained from the Healthcare Cost and Utilization Project (HCUP) Nationwide Inpatient Sample (NIS) database. The NIS, compiled by the Agency for Healthcare Research and Quality (Rockville, Maryland, USA), represents the largest allpayer inpatient administrative database in the United States. It contains data from over 1000 hospitals and more than 8 million hospital stays annually and is designed to approximate a $20 \%$ sample of all hospital discharges, which includes all nonfederal, short-term, general, and other specialty hospitals, including public hospitals and academic institutions. ${ }^{23}$ 


\section{Patient Selection}

The International Classification of Diseases, Ninth Revision (ICD-9) procedural coding terminology was used to identify all patients who underwent any type of IBR; all implant based and autologous techniques of breast reconstruction were included. To make the study population as similar as possible and minimize confounding medical variables, a cohort of 64 year-old women with private insurance was compared to a cohort of 66 year-old women with Medicare. Demographic data, IBR rates, and major complication rates were compared between these two cohorts over a 15-year period from 1998 to 2013. Trend over time were plotted for the years 1992 to 2013 to provide an overview of the years prior the WHCRA act.

Patients diagnosed with invasive breast cancer (ICD-9 diagnosis codes 174.0, 174.1, $174.2,174.3,174.4,174.5,174.6,174.8$ and 174.9) undergoing unilateral or bilateral mastectomy were included. Patients with multiple ICD-9 procedure codes or with an additional prophylactic mastectomy code were classified as having bilateral mastectomy. Implant-based breast reconstructions were defined as either placement of immediate silicone implant or tissue expander after mastectomy. Autologous breast reconstructions included both pedicled and free tissue options: latissimus dorsi flap (LD), pedicled transverse rectus abdominus muscle flap (pTRAM), free transverse rectus abdominus muscle flap (fTRAM), deep inferior epigastric perforator flap (DIEP), superficial inferior epigastric artery perforator flap (SIEA) or gluteal artery perforator flaps (GAP) (Supplemental Digital Content, Table 1). Male patients, those of unknown gender or age were excluded. Cases of breast reconstruction without concurrent mastectomy were considered delayed and were also excluded from analysis. 


\section{Analyzed Variables}

The analyzed variables included calendar year, geographical region, race, hospital status (teaching or non-teaching, and urban or rural), and number of hospital beds. The hospital's geographic region was broken down into four areas: Northeast (ME, NH, VT, MA, RI, CT, NY NJ, PA); Midwest (OH, IN, IL, MI, WI, MN, IA, MO, ND, SD, NE, KS); South (DE, MD, DC, VA, WV, NC, SC, GA, FL, KY, TN, AL, MS, AR, LA, OK, TX) and West (MT, ID, WY, CO, NM, AZ, UT, NV, WA, OR, CA, AK, HI).

Hospital teaching status is determined by NIS as those hospitals with American Medical Association-approved residency programs, or are members of the council of Teaching Hospitals, or have a ratio of full-time residents to beds of 0.25 or greater. ${ }^{24}$ The number of hospital beds was organized into groups determined by the Nationwide Inpatient Sample as: small (1 - 49 beds for rural hospitals, 1 - 99 for urban non-teaching hospitals, and 1 - 299 beds for urban teaching hospitals); medium (50 - 99 for rural hospitals, 100 - 199 for urban non-teaching hospitals, and 300 - 499 for urban teaching hospitals) and large (>100 for rural hospitals, >200 beds for urban non-teaching hospitals, and $>500$ for urban teaching hospitals). ${ }^{10,} 25$ Comorbidities identified using ICD-9 codes were used to calculate the Charlson Comorbidity Index (CCI). ${ }^{26}$ The $\mathrm{CCI}$ has been validated for administrative databases and has been used previously to examine the influence of comorbidity on breast cancer treatment and outcomes. ${ }^{26-28} \mathrm{CCl}$ score was divided into four groups; $\mathrm{CCl}$ core $0, \mathrm{CCl}$ score $1, \mathrm{CCl}$ score 2 and $\mathrm{CCl}$ score >=3. Post-operative complications were determined by using ICD-9 codes. The NIS does not contain information on patient outcomes after discharge since it records inpatient data only. Complications occurring after hospital discharge were therefore not captured in our analysis.

\section{Statistical Analysis}

The frequencies of categorical variables are expressed as a percentage of the group of origin. Categorical variables were compared using Chi-square analyses. Trends were analyzed using the Cochran-Armitage test. All analyses were performed using IBM SPSS Version 22.0 (IBM Corp., Armonk, N.Y.), and statistical significance was set at $p<0.05$.

\section{RESULTS}

During the study period, a total of 21,402 women with private insurance (age 64) and 25,568 women with Medicare (age 66) were diagnosed with invasive breast cancer and included in the study. Of these, $72.3 \%(n=15,469)$ within the privately insured cohort and $71.2 \%$ ( $n=18,194)$ within the Medicare cohort opted for mastectomy $(p=0.007)$. During the study period, rates of unilateral mastectomy decreased significantly while rates of bilateral mastectomy increased significantly in both groups. Unilateral 
mastectomy decreased from 96.8\% in 1992 to $49.1 \%$ in 2013 ( $p<0.001)$ in the privately insured group (Figure 1a). Similar trends were seen in Medicare cohort: Unilateral mastectomy decreased from 96.5\% in 1992 to 65.5\% in 2013 ( $p<0.001$ ) (Figure 1b). Bilateral mastectomy consequently increased from $3.5 \%$ to $50.9 \%$ in the privately insured cohort $(p<0.001$ ), and from $3.2 \%$ to $34.5 \%$ in the Medicare insured cohort ( $<<$ 0.001 ) between 1992 and 2013. (Figure 1a-b)

\section{Uni- and Bilateral Mastectomy in Private Insurance Group}

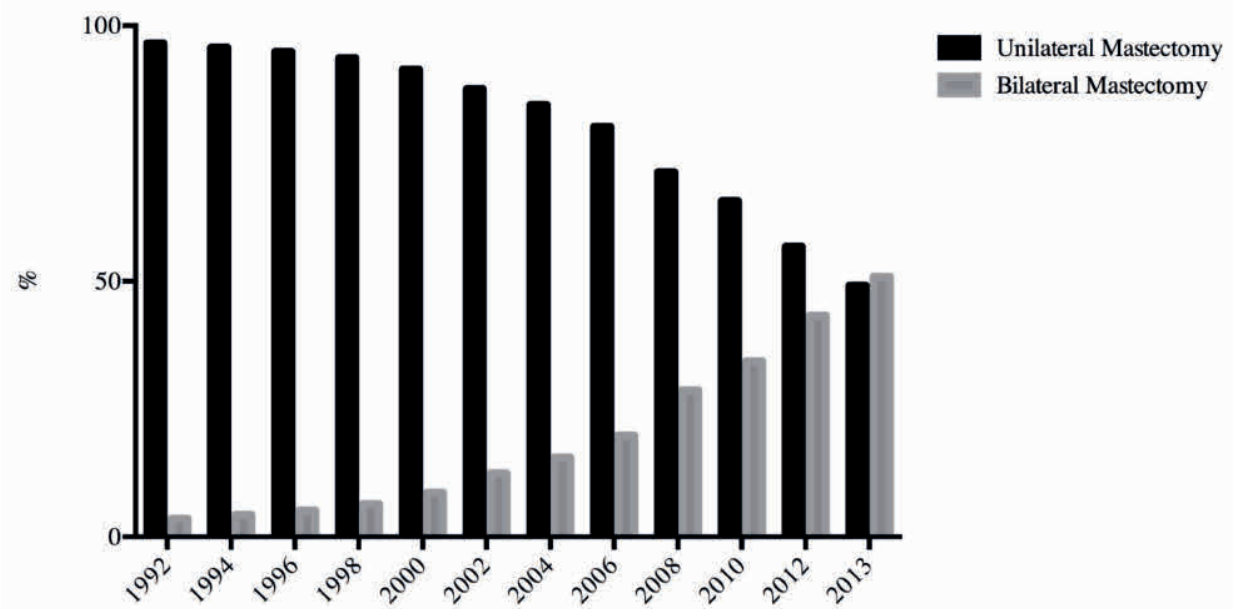

Figure 1. Rates of unilateral and bilateral mastectomy among (A) 64 year-old privately insured women and (B) 66 year-old Medicare insured women. Cochrane-Armitage test for trend $p<0.001$ in both cohorts.

\section{Uni - and Bilateral Mastectomy in Medicare Insurance Group}

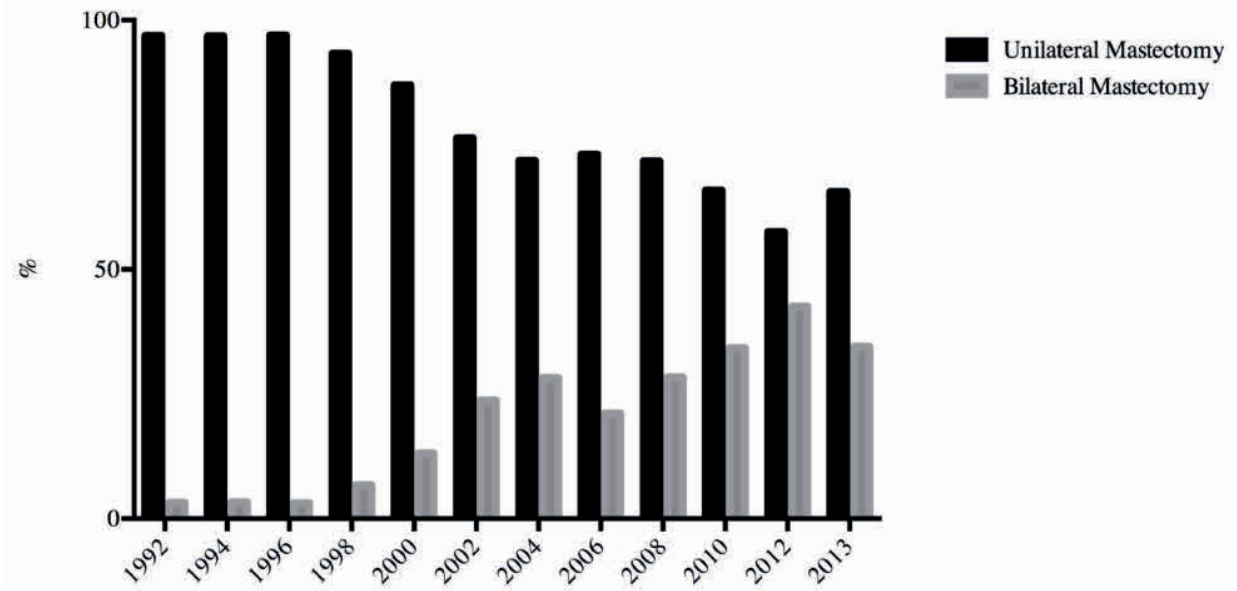

Figure 2. Rates of immediate breast reconstruction over study period. Cochrane-Armitage test for trend $p<$ 0.001 in both cohorts. 
Table 1. Summary of patient characteristics

\begin{tabular}{|c|c|c|c|c|c|}
\hline & \multicolumn{2}{|c|}{ Privately Insured } & \multicolumn{2}{|c|}{ Medicare Insured } & \multirow[b]{2}{*}{$\mathrm{p}$ value } \\
\hline & $n=$ & $(\%)$ & $n=$ & $(\%)$ & \\
\hline Breast cancer $(n)$ & 21,402 & & 25,568 & & \\
\hline Mastectomy & 15,469 & $(72.3)$ & 18,194 & $(71.2)$ & 0.007 \\
\hline Post-mastectomy IBR & 3,941 & $(25.5)$ & 3,213 & $(17.7)$ & 0.001 \\
\hline Type of IBR & & & & & 0.225 \\
\hline Implant & 3,212 & $(81.5)$ & 2,589 & $(80.6)$ & \\
\hline Autologous & 440 & $(11.2)$ & 353 & $(11.0)$ & \\
\hline Combined & 289 & $(7.3)$ & 271 & $(8.4)$ & \\
\hline Reconstruction & & & & & 0.001 \\
\hline Unilateral & 2,325 & $(59.0)$ & 2,107 & $(65.6)$ & \\
\hline Bilateral & 1,616 & $(41.0)$ & 1,106 & $(34.4)$ & \\
\hline Race & & & & & 0.001 \\
\hline Caucasian & 2,878 & $(73.0)$ & 2,330 & $(72.5)$ & \\
\hline Black & 210 & $(5.3)$ & 213 & $(6.6)$ & \\
\hline Hispanic & 142 & (3.6) & 134 & $(4.2)$ & \\
\hline Asian/PI & 87 & $(2.2)$ & 33 & $(1.0)$ & \\
\hline Other & 624 & $(15.8)$ & 502 & $(15.6)$ & \\
\hline Hospital Type & & & & & 0.004 \\
\hline Non-teaching & 1,542 & $(39.2)$ & 1,362 & $(42.6)$ & \\
\hline Teaching & 2,390 & $(60.8)$ & 1,836 & $(57.4)$ & \\
\hline Hospital Location & & & & & 0.040 \\
\hline Rural & 109 & $(2.8)$ & 116 & (3.6) & \\
\hline Urban & 3,822 & $(97.2)$ & 3,082 & $(96.4)$ & \\
\hline Hospital Bed-size & & & & & 0.025 \\
\hline Small & 409 & $(11.5)$ & 316 & $(10.9)$ & \\
\hline Medium & 715 & $(20.1)$ & 663 & $(22.8)$ & \\
\hline Large & 2,438 & $(68.4)$ & 1,923 & $(66.3)$ & \\
\hline Length of Stay (d) & & & & & 0.005 \\
\hline 0 & 9 & $(0.2)$ & 4 & $(0.1)$ & \\
\hline 1 & 1,219 & (30.9) & 1,085 & $(33.8)$ & \\
\hline 2 & 1,529 & $(38.8)$ & 1,252 & $(39.0)$ & \\
\hline 3 & 623 & $(15.8)$ & 498 & $(15.5)$ & \\
\hline $4+$ & 560 & $(14.2)$ & 373 & $(11.6)$ & \\
\hline Region & & & & & 0.001 \\
\hline Northeast & 1,178 & (29.9) & 877 & $(27.3)$ & \\
\hline Midwest & 918 & $(23.3)$ & 595 & $(18.5)$ & \\
\hline South & 1,023 & $(26.0)$ & 994 & (30.9) & \\
\hline West & 822 & (20.9) & 787 & $(23.2)$ & \\
\hline
\end{tabular}


Table 1 demonstrates the overall characteristics of patients who underwent postmastectomy IBR. Overall, $25.5 \%$ ( $n=3,941$ ) of the privately insured patients and $17.7 \%$ $(n=3,213)$ of the Medicare insured patients underwent IBR $(p<0.001)$. No significant differences were seen between the two groups with respect to type of reconstruction: implant, autologous or combined ( $p=0.225)$. The majority of women in both cohorts were Caucasian $(p<0.001)$, were operated on in an urban $(p<0.040)$, teaching hospital $(p<0.004)$, with a large bedsize $(p<0.025)$, and had a length of stay of 2 days ( $p=$ 0.005). Most of privately insured women underwent IBR in the Northeast region (29.9\%), while the most Medicare insured women underwent IBR in the Southern region $(30.9 \%)$ ( $p<0.001)$. No significant difference was seen between the groups in terms of complications or $\mathrm{CCl}(\mathrm{p}=0.444)$ (Table 2$)$.

Table 2. Comparison of complication rates

\begin{tabular}{|c|c|c|c|c|c|}
\hline & \multicolumn{2}{|c|}{ Privately Insured } & \multicolumn{2}{|c|}{ Medicare Insured } & \multirow[b]{2}{*}{$p$ value } \\
\hline & $\mathrm{n}=$ & $(\%)$ & $\mathrm{n}=$ & $(\%)$ & \\
\hline$\overline{\mathrm{CCl} \text { index }}$ & & & & & 0.444 \\
\hline 0 & 0 & $(0.0)$ & 0 & $(0.0)$ & \\
\hline 1 & 0 & $(0.0)$ & 0 & $(0.0)$ & \\
\hline 2 & 1,369 & $(34.7)$ & 1,144 & $(35.6)$ & \\
\hline$\geq 3$ & 2,572 & $(65.3)$ & 2,069 & $(64.4)$ & \\
\hline \multicolumn{6}{|l|}{ Complications } \\
\hline Seroma & 27 & $(0.7)$ & 10 & $(0.3)$ & 0.028 \\
\hline Hematoma & 73 & $(1.0)$ & 50 & $(1.6)$ & 0.015 \\
\hline Wound Dehiscence & 5 & $(0.1)$ & 5 & $(0.2)$ & 0.746 \\
\hline Wound Infection & 25 & $(0.6)$ & 19 & $(0.6)$ & 0.817 \\
\hline DVT & 5 & $(0.1)$ & 10 & $(0.3)$ & 0.090 \\
\hline Respiratory Failure & 15 & $(0.4)$ & 8 & $(0.2)$ & 0.328 \\
\hline Renal Insufficiency & 10 & $(0.3)$ & 9 & $(0.3)$ & 0.829 \\
\hline UTI & 15 & $(0.4)$ & 10 & $(0.3)$ & 0.621 \\
\hline Sepsis & 5 & $(0.1)$ & 0 & $(0.0)$ & 0.043 \\
\hline Pneumonia & 10 & $(0.3)$ & 5 & $(0.2)$ & 0.367 \\
\hline
\end{tabular}

Finally, when looking at the trend over time, IBR increased significantly in both privately insured women as well as in Medicare insured women (Figure 2). Immediate breast reconstruction increased from 3.9\% in 1992 to $47.2 \%$ in 2013 among privately insured patients ( $p$ 0.001). A similar upward trend was seen in Medicare insured women, with IBR rates increasing from 2.3\% in 1992 to $43.7 \%$ in 2013 ( $p<0.001$ ) (Figure 2). The difference in IBR rates between the two cohorts in demonstrated in Figure 3. After an initial increase in differences of IBR utilization to 2006 ( $p<0.001)$, a significant decline in different rates is observed op to 2013 ( $p<0.001)$. 
Immediate Breast Reconstruction Rates in

Private vs. Medicare Insurance Group

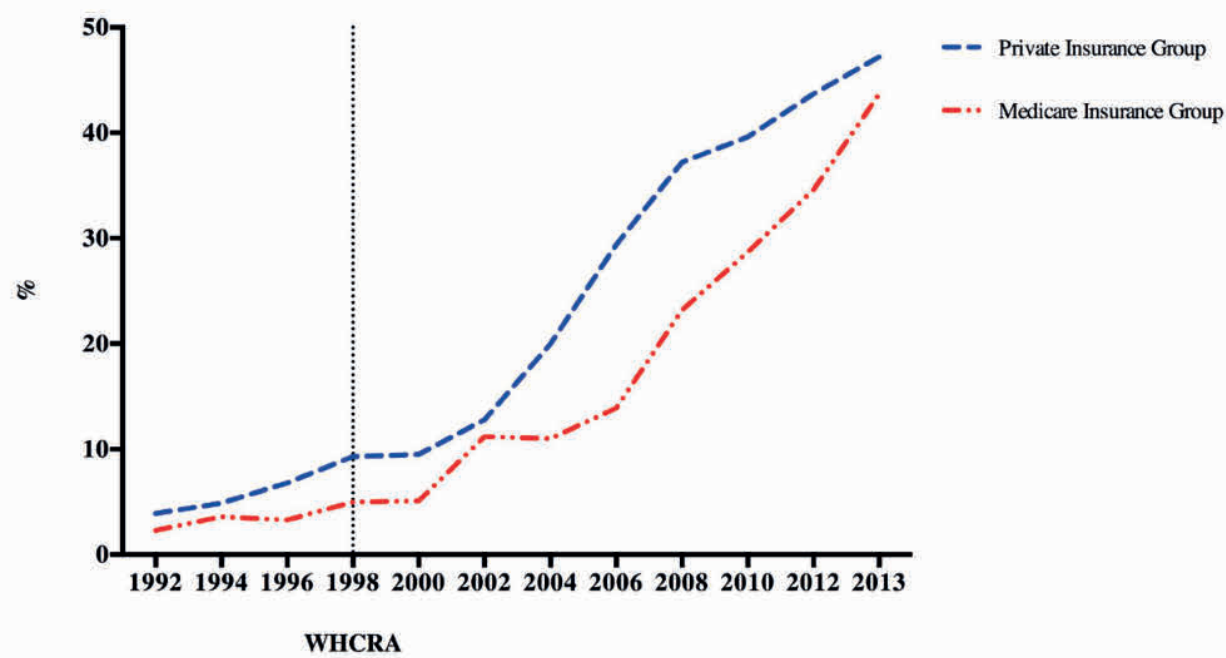

Figure 2. Rates of immediate breast reconstruction over study period. Cochrane-Armitage test for trend $p<0.001$ in both cohorts.

\section{Difference Private vs. Medicare IBR utilization}

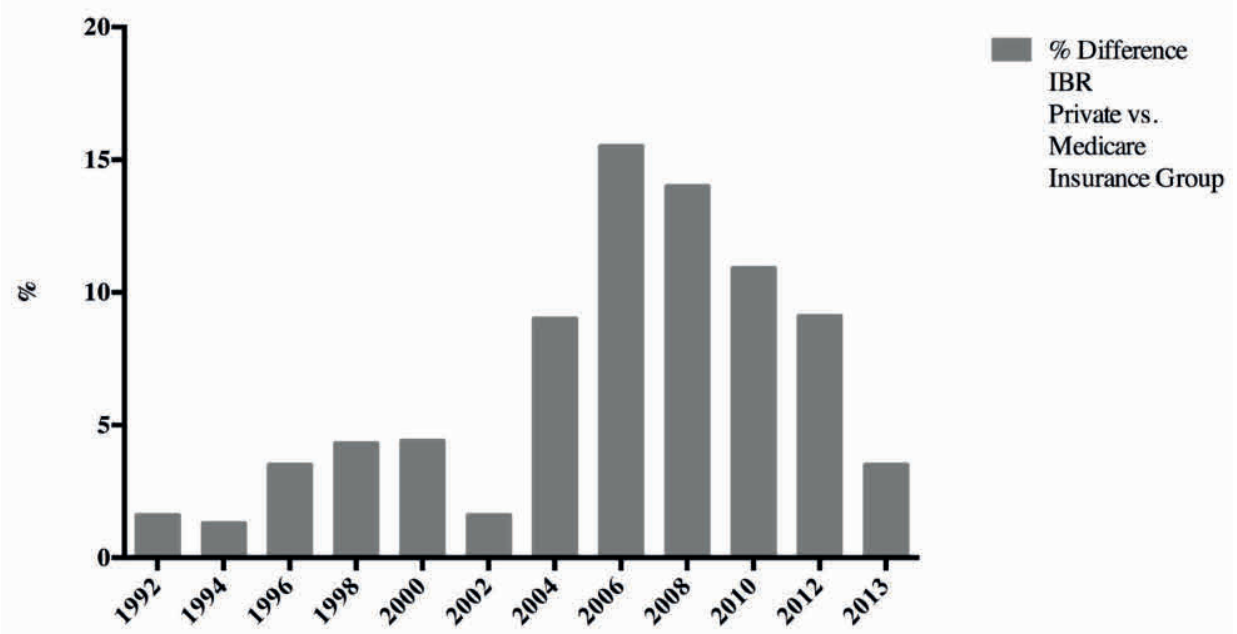

Figure 3. Differences of privately insured cohort immediate breast reconstruction rates compared to Medicare insured cohort immediate breast reconstruction rates. 


\section{DISCUSSION}

Since the passage of the Women's Health and Cancer Rights Act, which mandated coverage of breast reconstruction, there has been an increase in breast reconstruction among both privately and publicly insured patients. ${ }^{2,21,29-31}$ These findings were corroborated by our study. However, the majority of these papers reported disparities in Medicare insured women. A recent report by Wexelman et al., using the NIS database, shows that Medicare insured women are one and a half times less likely to undergo IBR when compared to private insured women. ${ }^{4}$ Reports by Roughton and Yang also demonstrated similarly low rates IBR among Medicare-insured women. 2,18 Unfortunately, these works do not account for the inherent differences in age and comorbidities between the typically younger patients with private insurance and the typically older Medicare population.

In this study, we examined and compared the trends of IBR between women with Medicare and those with privately insurance. To ensure similar groups and minimize bias with respect to inherent differences in medical comorbidities, only women age 64 years-old with private insurance and age 66 years-old with Medicare were included. Not only did this study show increased rates IBR over time in both groups, but that after an initial slower upward trend, the rate of IBR in Medicare insured women nearly equaled that among private insurance over the duration of this study (Figure 2). In 2013, the final year of this study period, IBR was performed in $47.2 \%$ of privately insured women versus $43.7 \%$ of women in the Medicare group (data not shown).

This study demonstrates that in recent years, there have been no significant differences in the receipt of IBR in both groups. One reason for the disparity in the literature may be due to the fact that women have been analyzed based on insurance type (Medicare versus private insurance) without regard for patient age and associated comorbidities. This is of particular import in the group of patients insured by Medicare where there exists a large bimodal distribution of very young and very old women, based on eligibility requirements for coverage. Unlike previous manuscripts, this study is the first to take into account and minimize the differences in comorbidities between these groups before analyzing the rates of IBR by creating matched cohorts.

There are likely many reasons for the observed increased rates of IBR among breast cancer patients. Passage of the WHCRA has lessened the financial barriers to this procedure among a large number of patients. Additionally, the feasibility of prophylactic mastectomy and reconstruction has brought to the public's attention by several prominent celebrities. In the future, further patient education will likely be a driver toward increased access to IBR as a favourable option for breast cancer patients. Of note, Congress has recently passed the Breast Cancer Patient Education Act of 2015, which will allocate more resources toward patient education regarding options for breast reconstruction following mastectomy. ${ }^{16}$ 
Recently, there has been much ongoing speculation among plastic surgeons in the literature that declining reimbursement rates may be limiting the reconstructive options offered to patients. ${ }^{32-35}$ To this end, Hernandez-Boussard et al. examined surgeon fee schedules using the national average Medicare physician reimbursement rates compared to the type of reconstruction chosen by patients. They observed a steady decrease in the rates of autologous breast reconstruction from 1998 to 2008 which closely paralleled a decrease in physician reimbursement over that same time period. ${ }^{1}$ Our study did not show a similar trend; but rather that regardless of insurance type, women are being offered IBR at ever increasing rates over a similar time period.

There are several limitations of this study that should be noted. Firstly, while data from the NIS is a reflection of the entire country and provides us valuable information on current practices in the United States, this study is retrospective in nature. Secondly, this study was only limited to patients undergoing breast reconstruction in an immediate fashion after mastectomy. Accordingly, as patients undergoing delayed reconstruction were excluded from this study, there is a cohort of women who ultimately had breast reconstruction that were not included in this study. Finally, we were unable to conduct a detailed assessment of non-surgical treatment, patient's background, and the decision-making processes. Nevertheless, this study provides a comprehensive overview of a matched cohort privately insured and Medicare insured women undergoing IBR.

\section{CONCLUSION}

In this study, we examined IBR utilization between a matched population based on insurance type, Medicare or private insurance. The results of this study demonstrate a significant increase in rates of IBR among women regardless of insurance type over the time period of this study. Moreover, after an initial slower upward trend, after a decade IBR in Medicare insured women approached nearly similar rates among those with private insurance. 


\section{FIGURE AND TABLE LEGEND}

Table 1. Summary of patient characteristics

Table 2. Comparison of complication rates

Figure 1. Rates of unilateral and bilateral mastectomy among (A) 64 year-old privately insured women and (B) 66 year-old Medicare insured women. Cochrane-Armitage test for trend $p<0.001$ in both cohorts

Figure 2. Rates of immediate breast reconstruction over study period. CochraneArmitage test for trend $p<0.001$ in both cohorts

Figure 3. Differences of privately insured cohort immediate breast reconstruction rates compared to Medicare insured cohort immediate breast reconstruction rates

Supplemental Digital Content, Table 1. ICD-9 procedure codes for mastectomy, implant-based and autologous breast reconstruction 


\section{SUPPLEMENT}

Supplemental Digital Content, Table 1. ICD-9 procedure codes for mastectomy, implant-based and autologous breast reconstruction

\begin{tabular}{|c|c|c|}
\hline Inclusion criteria & ICD-9 code & Description \\
\hline \multicolumn{3}{|l|}{ Mastectomy } \\
\hline & 85.40 & Mastectomy \\
\hline & v50.41 & Prophylactic removal of breast \\
\hline \multicolumn{3}{|c|}{ Unilateral Mastectomy } \\
\hline & 85.34 & Other unilateral subcutaneous mammectomy \\
\hline & 85.41 & Unilateral simple mastectomy \\
\hline & 85.43 & Unilateral extended simple mastectomy \\
\hline & 85.45 & Unilateral radical mastectomy \\
\hline & 85.47 & Unilateral extended radical mastectomy \\
\hline & 85.33 & $\begin{array}{l}\text { Unilateral subcutaneous mammectomy with synchronous } \\
\text { implant }\end{array}$ \\
\hline \multicolumn{3}{|c|}{ Bilateral Mastectomy } \\
\hline & 85.36 & Other bilateral subcutaneous mammectomy \\
\hline & 85.42 & Bilateral simple mastectomy \\
\hline & 85.44 & Bilateral extended simple mastectomy \\
\hline & 85.46 & Bilateral radical mastectomy \\
\hline & 85.48 & Bilateral extended radical mastectomy \\
\hline & 85.35 & $\begin{array}{l}\text { Bilateral subcutaneous mammectomy with synchronous } \\
\text { implant }\end{array}$ \\
\hline \multicolumn{3}{|c|}{ Breast reconstruction } \\
\hline \multicolumn{3}{|c|}{ Tissue Expander/Implant BR } \\
\hline & 85.33 & $\begin{array}{l}\text { Unilateral subcutaneous mammectomy with synchronous } \\
\text { implant }\end{array}$ \\
\hline & 85.35 & $\begin{array}{l}\text { Bilateral subcutaneous mammectomy with synchronous } \\
\text { implant }\end{array}$ \\
\hline & 85.53 & Unilateral breast implant \\
\hline & 85.54 & Bilateral breast implant \\
\hline & 85.95 & Insertion breast tissue expander \\
\hline & 85.96 & Removal breast tissue expander \\
\hline \multicolumn{3}{|l|}{ Autologous BR } \\
\hline & 85.71 & Latissimus dorsi myocutaneous flap (LD), pedicled \\
\hline & 85.72 & $\begin{array}{l}\text { Transverse rectus abdominis myocutaneous (TRAM) flap, } \\
\text { pedicled }\end{array}$ \\
\hline & 85.73 & Transverse rectus abdominis myocutaneous (TRAM) flap, free \\
\hline & 85.74 & Deep inferior epigastric artery perforator (DIEP) flap, free \\
\hline & 85.75 & Superficial inferior epigastric artery (SIEA) flap, free \\
\hline & 85.76 & Gluteal artery perforator (GAP) flap, free \\
\hline
\end{tabular}




\section{REFERENCES}

1. Hernandez-Boussard, T., Zeidler, K., Barzin, A., Lee, G., Curtin, C. Breast reconstruction national trends and healthcare implications. The breast journal 2013;19:463-469.

2. Yang, R. L., Newman, A. S., Lin, I. C., et al. Trends in immediate breast reconstruction across insurance groups after enactment of breast cancer legislation. Cancer 2013;119:2462-2468.

3. Offodile, A. C., 2nd, Tsai, T. C., Wenger, J. B., Guo, L. Racial disparities in the type of postmastectomy reconstruction chosen. The Journal of surgical research 2015;195:368-376.

4. Wexelman, B., Schwartz, J. A., Lee, D., Estabrook, A., Ma, A. M. Socioeconomic and geographic differences in immediate reconstruction after mastectomy in the United States. The breast journal 2014;20:339-346.

5. Mahmoudi, E., Giladi, A. M., Wu, L., Chung, K. C. Effect of federal and state policy changes on racial/ethnic variation in immediate postmastectomy breast reconstruction. Plastic and reconstructive surgery 2015;135:1285-1294.

6. Albornoz, C. R., Bach, P. B., Pusic, A. L., et al. The influence of sociodemographic factors and hospital characteristics on the method of breast reconstruction, including microsurgery: a U.S. population-based study. Plastic and reconstructive surgery 2012;129:1071-1079.

7. Jagsi, R., Jiang, J., Momoh, A. O., et al. Trends and variation in use of breast reconstruction in patients with breast cancer undergoing mastectomy in the United States. Journal of clinical oncology : official journal of the American Society of Clinical Oncology 2014;32:919-926.

8. Merchant, S. J., Goldstein, L., Kruper, L. L. Patterns and Trends in Immediate Postmastectomy Reconstruction in California: Complications and Unscheduled Readmissions. Plastic and reconstructive surgery 2015;136:10e-19e.

9. Polednak, A. P. Geographic variation in postmastectomy breast reconstruction rates. Plastic and reconstructive surgery 2000;106:298-301.

10. Kamali, P., Koolen, P. G., Ibrahim, A. M., et al. Analyzing Regional Differences over a 15-Year Trend of One-Stage versus Two-Stage Breast Reconstruction in 941,191 Postmastectomy Patients. Plastic and reconstructive surgery 2016;138:1e-14e.

11. Ibrahim, A. M., Shuster, M., Koolen, P. G., et al. Analysis of the National Surgical Quality Improvement Program database in 19,100 patients undergoing implant-based breast reconstruction: complication rates with acellular dermal matrix. Plastic and reconstructive surgery 2013;132:1057-1066.

12. Vargas, C. R., Ganor, O., Semnack, M., Lin, S. J., Tobias, A. M., Lee, B. T. Patient preferences in access to breast reconstruction. The Journal of surgical research 2015;195:412-417.

13. Ashraf, A. A., Colakoglu, S., Nguyen, J. T., et al. Patient involvement in the decision-making process improves satisfaction and quality of life in postmastectomy breast reconstruction. The Journal of surgical research 2013;184:665-670.

14. Kantak, N. A., Koolen, P. G., Martin, C., Tobias, A. M., Lee, B. T., Lin, S. J. Are patients with low body mass index candidates for deep inferior epigastric perforator flaps for unilateral breast reconstruction? Microsurgery 2015;35:421-427.

15. Sinno, H., Izadpanah, A., Thibaudeau, S., Christodoulou, G., Lin, S. J., Dionisopoulos, T. An objective assessment of the perceived quality of life of living with bilateral mastectomy defect. Breast 2013;22:168-172.

16. Breast Cancer Patient Education Act of 2015, H. R. 2540, 114th Congress (2015-2016). Available at: https://www.congress.gov/bill/114th-congress/house-bill/2540 Assessed October 15, 2016.

17. Christian, C. K., Niland, J., Edge, S. B., et al. A multi-institutional analysis of the socioeconomic determinants of breast reconstruction: a study of the National Comprehensive Cancer Network. Annals of surgery 2006;243:241-249.

18. Roughton, M. C., DiEgidio, P., Zhou, L., Stitzenberg, K., Meyer, A. M. Distance to a Plastic Surgeon and Type of Insurance Plan Are Independently Predictive of Postmastectomy Breast Reconstruction. Plastic and reconstructive surgery 2016;138:203e-211e. 
19. Kummerow, K. L., Du, L., Penson, D. F., Shyr, Y., Hooks, M. A. Nationwide trends in mastectomy for earlystage breast cancer. JAMA surgery 2015;150:9-16.

20. Miller, A., Chandru Kowdley, G. Breast reconstruction after mastectomy at an urban community-based program. The American surgeon 2012;78:1281-1284.

21. Hershman, D. L., Richards, C. A., Kalinsky, K., et al. Influence of health insurance, hospital factors and physician volume on receipt of immediate post-mastectomy reconstruction in women with invasive and non-invasive breast cancer. Breast cancer research and treatment 2012;136:535-545.

22. Kotwall, C., Brinker, C., Covington, D., et al. Local and national trends over a decade in the surgical treatment of ductal carcinoma in situ. American journal of surgery 2003;186:723-728; discussion 728-729.

23. U.S. Department of Health \& Human Services. Agency for Healthcare Research and Quality (HCUP). NIS description. Information available at: http://www.hcup-us.ahrq.gov/databases.jsp Assessed: June 15th 2015.

24. U.S. Department of Health \& Human Services. Agency for Healthcare Research and Quality (HCUP). NIS description of data elements. Available at: http://www.hcup-us.ahrq.gov/db/vars/h_bedsz/nisnote.jsp Assessed: June 15th 2015.

25. U.S. Department of Health \& Human Services. Agency for Healthcare Research and Quality (HCUP). NIS description of data elements. Available at: http://www.hcup-us.ahrq.gov/db/vars/hosp_bedsize/ nisnote.jsp. Accessed October 8, 2016.

26. Charlson, M. E., Pompei, P., Ales, K. L., MacKenzie, C. R. A new method of classifying prognostic comorbidity in longitudinal studies: development and validation. Journal of chronic diseases 1987;40:373-383.

27. Quan, H., Sundararajan, V., Halfon, P., et al. Coding algorithms for defining comorbidities in ICD-9-CM and ICD-10 administrative data. Medical care 2005;43:1130-1139.

28. Dehal, A., Abbas, A., Johna, S. Racial disparities in clinical presentation, surgical treatment and in-hospital outcomes of women with breast cancer: analysis of nationwide inpatient sample database. Breast cancer research and treatment 2013;139:561-569.

29. Albornoz, C. R., Bach, P. B., Mehrara, B. J., et al. A paradigm shift in U.S. Breast reconstruction: increasing implant rates. Plastic and reconstructive surgery 2013;131:15-23.

30. Shippee, T. P., Kozhimannil, K. B., Rowan, K., Virnig, B. A. Health insurance coverage and racial disparities in breast reconstruction after mastectomy. Women's health issues : official publication of the Jacobs Institute of Women's Health 2014;24:e261-269.

31. Reuben, B. C., Manwaring, J., Neumayer, L. A. Recent trends and predictors in immediate breast reconstruction after mastectomy in the United States. American journal of surgery 2009;198:237-243.

32. Silverstein, G. Physicians' perceptions of commercial and Medicaid managed care plans: a comparison. Journal of health politics, policy and law 1997;22:5-21.

33. Taylor, T. B. Threats to the health care safety net. Academic emergency medicine : official journal of the Society for Academic Emergency Medicine 2001;8:1080-1087.

34. Alderman, A. K., Storey, A. F., Nair, N. S., Chung, K. C. Financial impact of breast reconstruction on an academic surgical practice. Plastic and reconstructive surgery 2009;123:1408-1413.

35. Patel, A., Clune, J. E., Forte, A., Chang, C. C. The impact of the Medicare sustainable growth rate formula on reconstructive plastic surgery. Plastic and reconstructive surgery 2010;126:270e-271e. 



\section{Chapter}

\section{Trends In Immediate Breast Reconstruction and Early Complication Rates Among Older Women: A Big Data Analysis}

Parisa Kamali

Daniel Curiel

Charlotte L. van Veldhuisen

Alexandra E. M. Bucknor

Bernard T. Lee

Hinne A. Rakhorst

Samuel J. Lin 


\section{ABSTRACT}

\section{Background}

Although approximately $57 \%$ of breast cancer (BC) diagnoses are in older patients (>60 years), only $4.1 \%$ to $14 \%$ receives breast reconstruction (BR). This has been attributed to physician concerns about operative complications. This paper aims to: 1) analyze the 30-day complication rates in the older patient population undergoing immediate breast reconstruction (IBR), and 2) analyze links between complication type and category of reconstruction.

\section{Methods}

Using the ACS-NSQIP database (2005-2014), all women older than 60 years of age diagnosed with BC and DCIS were identified. IBR and complication rates were plotted for all ages. Patients were divided into those with and those without complications. Patient demographics and co-morbidities were compared. Complications within each type of reconstruction were analyzed.

\section{Results}

Of the 4,450 BC and 1,104 DCIS patients, 22.3\% (BC) and 20.9\% (DCIS) had complications. IBR decreased significantly with increased age ( $p<0.00$ in both cohorts), while complication rates remained stable across all ages ( $p=0.32$ in $B C, p=0.69$ in DCIS patients). Patients were well matched in terms of demographics.

\section{Conclusion}

The rates of breast reconstruction decrease with increasing age. Despite increasing age, associated complication rates in IBR patients remained stable.

\section{Synopsis}

This study analyzed the complication rates in the elderly patient population ( $>60$ years) undergoing immediate breast reconstruction and found that despite increasing age, associated complication rates in IBR patients remained stable in both BC as DCIS patients. 


\section{INTRODUCTION}

In 2015, approximately $57 \%$ of the 231,840 newly diagnosed invasive breast cancer (BC) cases and $49 \%$ of newly diagnosed Ductal Carcinoma In Situ (DCIS) cases were aged 60 years or older within the United States. ${ }^{1}$ With an estimated growth forecast of $57 \%$ from 2010 to 2030, older patients with early stage breast cancer constitute the most rapidly growing breast cancer demographic. ${ }^{2,3}$ Similar rates and epidemiological shifts are observed in other economically developed countries. ${ }^{4-7}$ Over the last decades, survival rates following breast cancer diagnosis have improved significantly due to advances in screening and treatment. ${ }^{8}$ As patients are living longer after breast cancer treatment, a stronger emphasis has been placed on improving quality of life. However, although treatment recommendations have been advocated for all women with early stage breast cancer, older women (defined here as those over 60 years of age) with breast cancer may be receiving differences in treatment. ${ }^{9-11}$

Post-mastectomy breast reconstruction (PMBR) is an important component of the comprehensive management of breast cancer patients. A large number of studies have demonstrated significant increase in self-esteem, body image and quality of life after breast reconstruction, in both young and older breast cancer survivors. ${ }^{11-13}$ After the implementation of Women's Health and Cancer Rights Act of 1998, a significant increase in the utilization of PMBR has been reported. ${ }^{14-17}$ Yet, although the large majority of breast cancer diagnoses is in older patients, only $4.1 \%$ to $14 \%$ of this population receives PMBR. ${ }^{11,18-24}$ The low rates of PMBR have been attributed to physician judgment of operative risk, the belief that reconstruction is unnecessary in this age group and patient concerns about operative complications. ${ }^{5,25}$

Immediate breast reconstruction (IBR) at the time of mastectomy has taken on greater popularity and is increasingly considered as standard of care. ${ }^{26-31}$ Compared to a delayed approach, IBR offers the potential benefit of fewer operations, decreased costs, better esthetic outcomes, and reduced psychological distress for the patient. ${ }^{26,32-34} \mathrm{~A}$ variety of reports have looked at post-procedure complication rates in patients undergoing breast reconstruction; however, they have frequently combined all women over 65 years of age into one group, or excluded women aged over 60 years from their analysis, limiting thorough evaluation of this group. ${ }^{5,35-37}$ Significant differences exist within this group of patients; although some may suffer with worsening health, many older women are still active and healthy. The aim of this paper is therefore 1) to analyze the at 30-day complication rates in the younger and older patient population undergoing IBR, 2) and to analyze possible links between complication type and category of reconstruction in the older patient population. 


\section{METHODS}

A retrospective analysis of The American College of Surgeons National Surgery Quality Improvement Program (ACS-NSQIP) database was performed for the years 2005 to 2014. The ACS-NSQIP provides prospectively collected clinical data on patients undergoing surgical procedures in hospitals across North America, and provides validated, 30-day surgical outcomes. All women diagnosed with invasive breast cancer (BC) or Ductal Carcinoma In Situ (DCIS) (International Classification of Disease, Ninth Edition (ICD-9) diagnosis codes 174.0 - 174.9 and 233.0) undergoing mastectomy and IBR were identified and included. Procedures were identified using Current Procedural Terminology (CPT) codes. Immediate breast reconstruction is defined as placement of tissue expander or permanent implant, autologous flap reconstruction, or combined (autologous and implant-based) reconstruction concurrent with mastectomy (Table 1). Patients without documented age, male patients, those undergoing outpatient procedure and those with metastatic disease were excluded.

Table 1. Current Procedural Terminology (CPT) codes for mastectomy and immediate breast reconstruction

\begin{tabular}{ll}
\hline National Surgical Quality Improvement Project (NSQIP) \\
\hline CPT code & Description \\
\hline 19303 & Mastectomy, simple, complete \\
19304 & Mastectomy, subcutaneous \\
19305 & Mastectomy, radical, including pectoral muscles, axillary lymph nodes \\
19306 & Mastectomy, radical, including pectoral muscles, axillary and internal mammary lymph \\
19307 & nodes (urban type operation) \\
19180 & minor muscle, but excluding pectoralis major muscle. \\
19182 & Subcutaneous mastectomy \\
19200 & Radical mastectomy \\
19220 & Urban type mastectomy \\
19240 & Modified radical mastectomy \\
\hline 19340 & Implant-based breast reconstruction \\
\hline 19342 & $\begin{array}{l}\text { Immediate insertion of breast prosthesis following mastopexy, mastectomy or in } \\
\text { reconstruction }\end{array}$ \\
& $\begin{array}{l}\text { Delayed insertion of breast prosthesis following mastoplexy, mastectomy, or in } \\
\text { reconstruction }\end{array}$ \\
& Breast reconstruction, immediate or delayed, with tissue expander, including subsequent \\
\end{tabular}




\begin{tabular}{|c|c|}
\hline \multicolumn{2}{|c|}{ National Surgical Quality Improvement Project (NSQIP) } \\
\hline CPT code & Description \\
\hline & Autologous breast reconstruction \\
\hline 19361 & Breast reconstruction with latissimus dorsi flap, without prosthetic implant \\
\hline 19364 & Breast reconstruction with free flap \\
\hline 19367 & $\begin{array}{l}\text { Breast reconstruction with transverse rectus abdominis myocutaneous flap (TRAM), single } \\
\text { pedicle, including closure of donor site; }\end{array}$ \\
\hline 19368 & $\begin{array}{l}\text { Breast reconstruction with transverse rectus abdominis myocutaneous flap (TRAM), single } \\
\text { pedicle, including closure of donor site; with microvascular anastomosis (supercharging) }\end{array}$ \\
\hline 19369 & $\begin{array}{l}\text { Breast reconstruction with transverse rectus abdominis myocutaneous flap (TRAM, double } \\
\text { pedicle, including closure of donor site) }\end{array}$ \\
\hline
\end{tabular}

We collected information on patient age, race, American Society of Anesthesiology (ASA) physical status, smoking status, body mass index (BMI, $>30 \mathrm{~kg} / \mathrm{m}^{2}$ defined obesity), presence of diabetes, COPD, hypertension, bleeding disorder, receipt of chemotherapy 30 days preoperatively, receipt of radiation therapy 90 days preoperatively, total operation time (minutes) and total length of stay (days). Several other endpoint outcome variables were obtained by combining defined NSQIP variables. Complications included those that were wound-related, infectious, respiratory, bleeding, thromboembolic, cardiac, renal and other miscellaneous complications.

Invasive breast cancer patients and DCIS patients were analyzed separately. Immediate breast reconstruction and complication rates were plotted for all ages $(\leq 39$, $40-44,45-49,50-54,55-59,60-64,65-69,70-74,75-79$, and $\geq 80$ years). Overall characteristics were only analyzed in women older than 60 years of age. This cohort was divided into two groups: those with and those without complications. We compared patient demographics and co-morbidities between those with and without complications. Specific complications within each type of reconstruction were analyzed.

\section{Statistical analysis}

Frequency counts and percentages were calculated for categorical variables and comparisons were made using the Pearson Chi-Square test. Medians and standard deviations were calculated for continuous variables. Cochrane-Armitage test for trend was utilized for trend analysis. All statistical analyses were performed using IBM SPSS version 22.0 (IBM Corp., Armonk, NY) and significance was set at $\mathrm{p}<0.05$. 


\section{RESULTS}

Invasive breast cancer patients

Figure 1 demonstrates IBR and complications trend by age. Immediate breast reconstruction decreased significantly from $65.5 \%$ in women aged $\leq 39$ years to $3.0 \%$ in women aged $\geq 80$ years $(p<0.000)$. Post-operative complication rates remained stable across all ages $(p=0.32)$.

Immediate breast reconstruction and complication rates in breast cancer patients

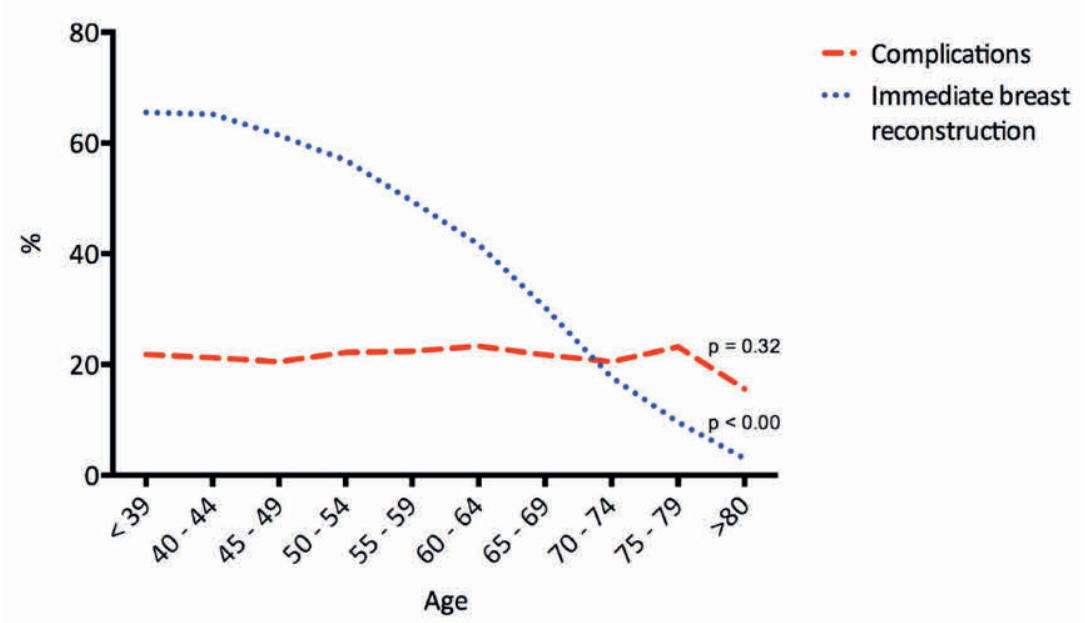

Figure 1. Immediate breast reconstruction and complication rates in invasive breast cancer patients

Table 2 demonstrates the overall characteristics of all women aged over 60. During the study period, a total of 4,450 patients diagnosed with invasive breast cancer underwent IBR. Of these, 3,457 (77.7\%) had no complications (NCMPL) and 993 (22.3\%) patients had one or more complications (CMPL). Patients were well matched in terms of age, race, smoking status, diabetes, history of COPD, hypertension, bleeding disorder, and preoperative chemo- and radiation therapy. The majority of patients in both cohorts was Caucasian (78.2\% vs $76.8 \%$ in CMPL vs. NCMPL respectively) aged $60-65$ (52.0\% vs $49.0 \%$ in CMPL vs. NCMPL respectively). Significant differences were observed between patients in both groups with regard to type of reconstruction $(p<0.000)$. Moreover, women with post-operative complications were significantly more likely to have a higher ASA-classification ( $p<0.00$ ), be obese $(37.0 \%$ vs. $32.2 \%$ in CMPL vs. NCMPL respectively, $p<0.000)$, have longer operation times $(258.7 \pm 222.0$ vs. $224.4 \pm 199.0$ minutes in CMPL vs. NCMPL respectively, $p<0.000)$ and a longer length of stay $(2.6 \pm$ 4.7 vs. $2.2 \pm 2.7$ days in CMPL vs. NCMPL respectively, $p<0.001$ ). 
Table 2. Overall characteristics invasive breast cancer patients

\begin{tabular}{|c|c|c|c|c|c|}
\hline & \multicolumn{2}{|c|}{ No complication } & \multicolumn{2}{|c|}{ Complication } & \multirow[t]{2}{*}{$\mathrm{p}$-value } \\
\hline & $n=$ & $\%$ & $n=$ & $\%$ & \\
\hline Total $(n=)$ & 3,457 & $(77.7)$ & 993 & $(22.3)$ & \\
\hline \multicolumn{6}{|l|}{ Age } \\
\hline $60-65$ & 1,694 & $(49.0)$ & 516 & $(52.0)$ & 0.259 \\
\hline $65-70$ & 1,051 & $(30.4)$ & 291 & $(29.3)$ & \\
\hline $70-75$ & 451 & $(13.0)$ & 116 & $(11.7)$ & \\
\hline $75-80$ & 185 & $(5.4)$ & 56 & $(5.6)$ & \\
\hline$\geq 80$ & 76 & $(2.2)$ & 14 & $(1.4)$ & \\
\hline Immediate breast reconstruction & & & & & 0.000 \\
\hline Implant & 2,702 & $(78.2)$ & 716 & $(72.1)$ & \\
\hline Autologous & 545 & $(15.8)$ & 231 & $(23.3)$ & \\
\hline Combined & 210 & $(6.1)$ & 46 & $(4.6)$ & \\
\hline Race & & & & & 0.282 \\
\hline White & 2,705 & $(78.2)$ & 763 & $(76.8)$ & \\
\hline Black & 242 & $(7.0)$ & 85 & $(8.6)$ & \\
\hline Hispanic & 139 & $(4.0)$ & 33 & (3.3) & \\
\hline Asian/PI & 88 & $(2.5)$ & 32 & $(3.2)$ & \\
\hline Other & 283 & $(8.2)$ & 80 & $(8.1)$ & \\
\hline ASA classification & & & & & 0.000 \\
\hline 1 & 83 & $(2.4)$ & 24 & $(2.4)$ & \\
\hline 2 & 2,289 & $(66.2)$ & 580 & $(58.4)$ & \\
\hline 3 & 1,068 & (30.9) & 378 & $(38.1)$ & \\
\hline 4 & 17 & $(0.5)$ & 11 & $(1.1)$ & \\
\hline Smoker & 291 & $(8.4)$ & 99 & $(10.0)$ & 0.127 \\
\hline BMI $\left(\mathrm{kg} / \mathrm{m}^{2}\right)$ & & & & & 0.000 \\
\hline$<29$ & 2,330 & $(67.8)$ & 622 & $(63.0)$ & \\
\hline$>30$ & 1,106 & $(32.2)$ & 365 & $(37.0)$ & \\
\hline Diabetes & 345 & $(10.0)$ & 112 & $(11.3)$ & 0.235 \\
\hline History of COPD & 68 & $(2.0)$ & 27 & $(2.7)$ & 0.150 \\
\hline Hypertension & 1,658 & $(48.0)$ & 505 & (50.9) & 0.110 \\
\hline Bleeding disorder & 31 & $(0.9)$ & 16 & $(1.6)$ & 0.052 \\
\hline Chemotherapy 30 days preop & 85 & $(4.2)$ & 6 & $(3.0)$ & 0.404 \\
\hline Missing & 2,209 & $(49.6)$ & & & \\
\hline Radiation 90 days preop & 12 & $(0.6)$ & 2 & $(1.0)$ & 0.495 \\
\hline Missing & 2,210 & $(49.7)$ & & & \\
\hline Total OR time (mean/SD) (min) & 224.4 & & 258. & & 0.000 \\
\hline Length of stay (mean/SD) (day) & $2.2 \pm 2$ & & $2.6 \pm$ & & 0.001 \\
\hline
\end{tabular}




\section{Chapter 5}

Table 3 demonstrates the type of complication stratified by reconstruction type. Women undergoing autologous breast reconstruction had a significantly higher rate of wound complications (5.5\% vs. $3.6 \%$ vs. $2.7 \%$ in autologous vs. implant vs. combined respectively, $p=0.023$ ), infectious complications ( $1.4 \%$ vs. $3.4 \%$ vs. $1.2 \%$ in autologous vs. implant vs. combined respectively, $p=0.001)$, bleeding complication $(0.6 \%$ vs. $7.5 \%$ vs. $0.4 \%$ in autologous vs. implant vs. combined respectively, $p<0.000$ ), and thromboembolic complication $(0.5 \%$ vs. $1.3 \%$ vs. $0.4 \%$ in autologous vs. implant vs. combined respectively, $p=0.037)$. No significant differences were seen in respiratory, cardial, renal and miscellaneous complications.

Table 3. Complication subtype stratified by reconstruction type in invasive breast cancer patients

\begin{tabular}{|c|c|c|c|c|}
\hline & \multicolumn{4}{|c|}{ Immediate breast reconstruction } \\
\hline & $\begin{array}{l}\text { Implant } \\
\mathrm{n}(\%)\end{array}$ & $\begin{array}{l}\text { Autologous } \\
\mathrm{n}(\%)\end{array}$ & $\begin{array}{l}\text { Combined } \\
\mathrm{n}(\%)\end{array}$ & p-value \\
\hline Total $(n=)$ & 3,418 & 776 & 256 & \\
\hline \multicolumn{5}{|c|}{ Type of complication } \\
\hline Wound & $122(3.6)$ & $43(5.5)$ & $7(2.7)$ & 0.023 \\
\hline Infectious & $47(1.4)$ & $26(3.4)$ & $3(1.2)$ & 0.001 \\
\hline Respiratory & $6(0.2)$ & $4(0.5)$ & $0(0.0)$ & 0.144 \\
\hline Bleeding & $22(0.6)$ & $58(7.5)$ & $1(0.4)$ & 0.000 \\
\hline Thromboembolic & $17(0.5)$ & $10(1.3)$ & $1(0.4)$ & 0.037 \\
\hline Cardial & $1(0.0)$ & $1(0.1)$ & $0(0.0)$ & 0.468 \\
\hline Renal & $0(0.0)$ & $0(0.0)$ & $0(0.0)$ & - \\
\hline Other & $550(16.1)$ & $151(19.5)$ & $39(15.2)$ & 0.062 \\
\hline
\end{tabular}

\section{DCIS patients}

Figure 2 demonstrates IBR and complication rates plotted by age. Immediate breast reconstruction was performed in $80.3 \%$ of women aged younger than 39 years, and decreased significantly as age increased to $3.1 \%$ in women older than 80 years ( $p<$ $0.00)$. Complication rates remained stable across all age groups $(p=0.69)$. 


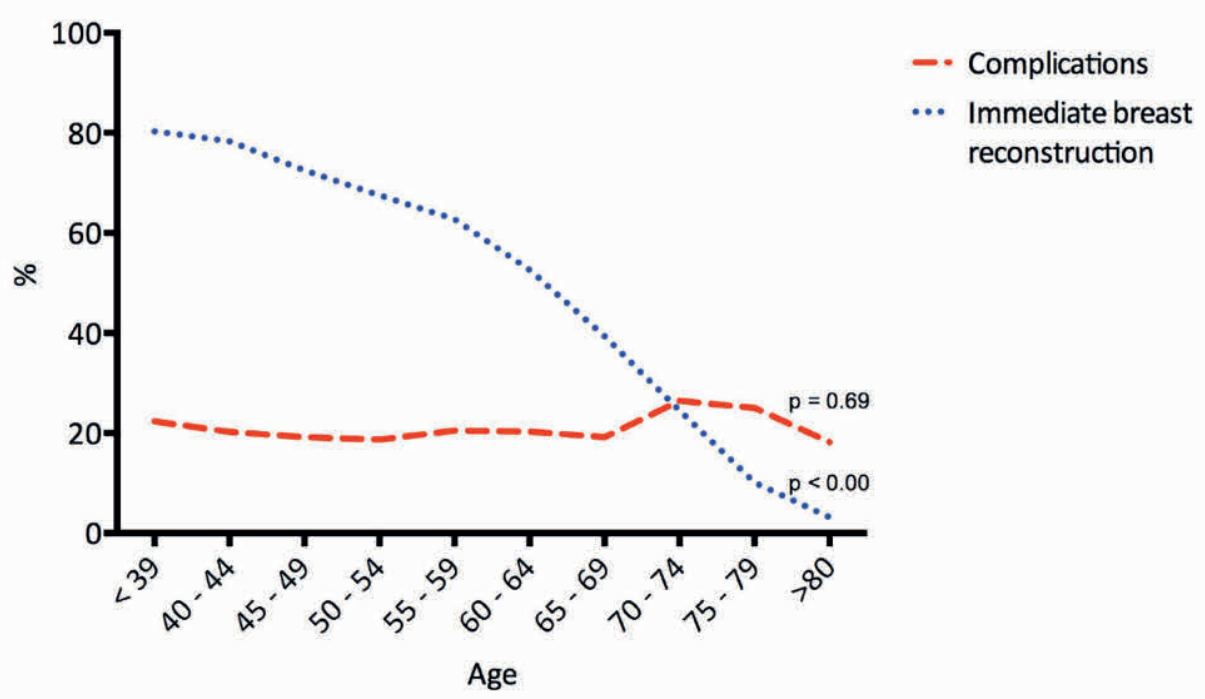

Figure 2. Immediate breast reconstruction and complication rates in DCIS patients

Table 4 demonstrates the overall characteristics of DCIS patients. Of the 1,104 DCIS patients who underwent IBR, 79.1\% ( $n=872)$ had no complications and $20.9 \%(n=231)$ had one or more complications. No significant differences were seen between the two cohorts in terms of age, race, smoking status, diabetes, COPD or length of stay. Of women experiencing complications, a significantly higher rate had undergone autologous breast reconstruction $(24.6 \%$ vs. $17.1 \%$ in CMPL vs. NCMPL, respectively, $p=$ 0.013). The majority of women in both cohorts was Caucasian (67.1\% vs. $72.7 \%$ in CMPL vs. NCMPL respectively) and were aged $60-65$ (50.2\% vs. 52.2\% in CMPL vs. NCMPL respectively). Women experiencing post-operative complications were significantly more likely to have a higher ASA-classification ( $p<0.028)$, BMI over $30 \mathrm{~kg} / \mathrm{m}^{2}(40.3 \% \mathrm{vs}$. $31.9 \%$ in CMPL vs. NCMPL respectively, $p<0.018)$, hypertension $(52.4 \%$ vs. $44.1 \%$, in CMPL vs. NCMPL respectively, $p<0.025)$, bleeding disorder $(2.2 \%$ vs. $0.3 \%$ in CMPL vs. NCMPL respectively, $p<0.004)$ and longer operating time $(246.6 \pm 166.1$ vs. $220.5 \pm$ 118.3 minutes in CMPL vs. NCMPL respectively, $p=0.007$ ). 
Table 4. Overall characteristics in DCIS patients

\begin{tabular}{|c|c|c|c|c|c|}
\hline & \multicolumn{2}{|c|}{ No complication } & \multicolumn{2}{|c|}{ Complication } & \multirow[t]{2}{*}{ p-value } \\
\hline & $\mathrm{n}=$ & $\%$ & $\mathrm{n}=$ & $\%$ & \\
\hline Total $(n=)$ & 872 & $(79.1)$ & 231 & $(20.9)$ & \\
\hline \multicolumn{5}{|l|}{ Age } & 0.431 \\
\hline $60-65$ & 456 & $(52.2)$ & 116 & $(50.2)$ & \\
\hline $65-70$ & 269 & $(30.8)$ & 64 & $(27.7)$ & \\
\hline $70-75$ & 106 & $(12.1)$ & 38 & $(16.5)$ & \\
\hline $75-80$ & 33 & $(3.8)$ & 11 & $(4.8)$ & \\
\hline$\geq 80$ & 9 & $(1.0)$ & 2 & $(0.9)$ & \\
\hline \multicolumn{5}{|l|}{ Immediate breast reconstruction } & 0.013 \\
\hline Implant & 678 & $(77.7)$ & 158 & $(68.4)$ & \\
\hline Autologous & 149 & $(17.1)$ & 57 & $(24.7)$ & \\
\hline Combined & 46 & $(5.3)$ & 16 & (6.9) & \\
\hline \multicolumn{5}{|l|}{ Race } & 0.352 \\
\hline White & 635 & $(72.7)$ & 155 & $(67.1)$ & \\
\hline Black & 67 & (7.7) & 24 & $(10.4)$ & \\
\hline Hispanic & 37 & $(4.2)$ & 9 & (3.9) & \\
\hline Asian/PI & 21 & $(2.4)$ & 9 & (3.9) & \\
\hline Other & 113 & (12.9) & 34 & $(14.7)$ & \\
\hline \multicolumn{5}{|l|}{ ASA classification } & 0.028 \\
\hline 1 & 36 & $(4.1)$ & 6 & $(2.6)$ & \\
\hline 2 & 602 & $(69.0)$ & 145 & $(62.8)$ & \\
\hline 3 & 234 & $(26.8)$ & 78 & $(33.8)$ & \\
\hline 4 & 1 & $(0.1)$ & 2 & $(0.9)$ & \\
\hline Smoker & 53 & $(6.1)$ & 16 & (6.9) & 0.633 \\
\hline \multicolumn{5}{|l|}{ BMI $\left(\mathrm{kg} / \mathrm{m}^{2}\right)$} & 0.018 \\
\hline$<29$ & 590 & $(68.1)$ & 138 & $(59.7)$ & \\
\hline$>30$ & 277 & (31.9) & 93 & $(40.3)$ & \\
\hline Diabetes & 94 & $(10.8)$ & 27 & $(11.7)$ & 0.690 \\
\hline History of COPD & 9 & $(1.0)$ & 5 & $(2.2)$ & 0.171 \\
\hline Hypertension & 385 & $(44.1)$ & 121 & $(52.4)$ & 0.025 \\
\hline Bleeding disorder & 3 & $(0.3)$ & 5 & $(2.2)$ & 0.004 \\
\hline Total OR time (mean/SD) (min) & \multicolumn{2}{|c|}{$220.5 \pm 118.3$} & \multicolumn{2}{|c|}{$246.6 \pm 166.1$} & 0.007 \\
\hline Length of stay (mean/SD) (day) & \multicolumn{2}{|c|}{$2.3 \pm 3.0$} & \multicolumn{2}{|c|}{$2.5 \pm 1.7$} & 0.474 \\
\hline
\end{tabular}

Subtypes of complications stratified by reconstruction type is demonstrated in Table 5 . Patients were well matched in terms of wound, infectious, respiratory, cardiac complications, renal and miscellaneous complications. Women with autologous reconstruction had higher bleeding complications ( $4.9 \%$ vs. $0.4 \%$ vs. $3.2 \%$ in autologous vs. implant vs. combined respectively, $p<0.000)$ and thromboembolic complications (3.4\% vs. $0.5 \%$ vs. $3.2 \%$ in autologous vs. implant vs. combined respectively, $p=0.001$ ). 
Table 5. Complication subtype stratified by reconstruction type in DCIS patients

\begin{tabular}{|c|c|c|c|c|}
\hline & \multicolumn{4}{|c|}{ Immediate breast reconstruction } \\
\hline & $\begin{array}{l}\text { Implant } \\
\mathrm{n}(\%)\end{array}$ & $\begin{array}{l}\text { Autologous } \\
\mathrm{n}(\%)\end{array}$ & $\begin{array}{l}\text { Combined } \\
\mathrm{n}(\%)\end{array}$ & $p$-value \\
\hline Total $(n=)$ & 836 & 206 & 63 & \\
\hline \multicolumn{5}{|c|}{ Type of complication } \\
\hline Wound & $34(4.1)$ & $14(6.8)$ & $3(4.8)$ & 0.246 \\
\hline Infectious & $7(0.8)$ & $4(1,9)$ & $0(0.0)$ & 0.259 \\
\hline Respiratory & $0(0.0)$ & $1(0.5)$ & $0(0.0)$ & 0.113 \\
\hline Bleeding & $3(0.4)$ & $10(4.9)$ & $2(3.2)$ & 0.000 \\
\hline Thromboembolic & $4(0.5)$ & $7(3.4)$ & $2(3.2)$ & 0.001 \\
\hline Cardial & $1(0.1)$ & $0(0.0)$ & $0(0.0)$ & 0.852 \\
\hline Renal & $0(0.0)$ & $0(0.0)$ & $0(0.0)$ & - \\
\hline Other & $116(13.9)$ & 34 (16.5) & $11(17.7)$ & 0.486 \\
\hline
\end{tabular}

\section{DISCUSSION}

Although treatment recommendations have been advocated for all women with breast cancer, many older women with breast cancer do not undergo post-mastectomy breast reconstruction. In this study, we examined the rates of IBR and complications within the older population. This study found that rates of immediate breast reconstruction decreased significantly with increasing age for both invasive breast cancer and DCIS patients. Importantly, complication rates remained similar throughout all age groups.

Our findings are consistent with other recent studies that have examined the trends in IBR following mastectomy. Naoura et al..$^{38}$ found that IBR among DCIS patients was more common for women under the age of 50 compared to women over 50. Similarly Lang et al. ${ }^{19}$ found that increasing age was inversely correlated with IBR among patients with invasive breast cancer ranging from stages I to III. These findings are supported by other studies showing that rates of IBR drop significantly when patients are 60 years of age or older. ${ }^{39,40}$

This study found that the 30-day post-IBR complication rates for patients with invasive breast cancer and DCIS, respectively, were $21.6 \%$ and $20.0 \%$. Moreover, the complication rate did not vary significantly across all the age groups that we examined ( $p=0.47$ invasive breast cancer, $p=0.12$ DCIS). The incidence of post-operative complications in the literature is highly variable. A recent systematic review identified ten studies reporting on complications in those over 60 years undergoing postmastectomy reconstruction, some in comparison with a younger cohort, with a range of $6.8 \%$ - $77 \%$ for older patients and $14.2 \%$ - $49.8 \%$ in younger women. In line with our results, Fisher et al. ${ }^{21,41}$ reported that advanced age not to be a predictor for 
complication rates after immediate breast reconstruction. More recently, Santosa et al. ${ }^{42}$ prospectively analyzed complication rates in patients undergoing immediate vs. delayed breast reconstruction with follow-up period of 2 years. They grouped the patients into three groups: $<45$ years, $45-60$ years and $>60$ years of age, and found that increased age does not affect breast reconstruction complication. However, this study, like others mentioned before, grouped all elderly patients over the age of 60 as one.

Our findings suggest that immediate breast reconstruction in elderly breast cancer patients may be a viable option with an expected complication rate comparable to the rate among younger patients. However, immediate breast reconstruction rates decrease significantly with increased age. One reason for the decline in IBR in older women may lie in surgeon perceptions of age and, consequently, a reluctance to discuss this option with patients. A survey of 61 older women undergoing breast reconstruction after mastectomy found that the subject of IBR was only discussed with 5/31 patients who ultimately underwent DBR, while all of them felt the topic should have been raised.(13) Actual and physiological age may be vastly different and other parameters should be use to better determine surgical safety, such as the Pre-operative Assessment of Cancer in the Elderly (PACE) tool, which is used to more accurately estimate operative risk in older patients, based on information about their co-morbidities and functional status. ${ }^{43}$

In addition to comprehensive pre-operative assessment of patient-related risk, when considering the best surgical course of action for patients, treating surgeons may also consider specific operative risks involved, including minimization of the duration of surgery, prevention of infection and restriction of blood loss - to which older patients are particularly vulnerable. Our observed finding that higher complications rates are associated with autologous breast reconstruction is supported by the literature: recent studies using national databases have found that post-operative complications are more frequently associated with autologous breast reconstruction, both within the first 30 days post-operation and even two years later, patients who have undergone autologous IBR carry a $9.5 \%$ risk of wound complications, compared to $4.4 \%$ for implant-based reconstruction and $2.3 \%$ for mastectomy-only patients. ${ }^{21,44}$

The strength of the current study includes its granular overview of both complication rates and immediate breast reconstruction rates over age. Moreover, by not grouping all patients over the age of 60 as one group, we provide a more comprehensive overview of this emerging patient population. Limitations of this study are primarily related to the database from which the data was collected. The ACS-NSQIP data includes only 30-day outcome and may therefore not portray the true picture of post-operative complications including the long-term outcomes after breast reconstruction. Additionally, occurrence breast reconstruction specific complications such as fat necrosis, hematoma, and seroma are not captured by the ACS-NSQIP. Moreover, as with any other large-volume dataset, the ACS-NSQIP is subject to observer bias and underreporting. Although the ACS-NSQIP includes a large number of both 
university and private hospitals, participation of hospitals is voluntary and it is not possible to completely eliminate selection bias.

\section{CONCLUSION}

These findings may have important implications for the surgeons who perform breast reconstruction and those who care for breast cancer patients. This study found that despite increasing age, associated complication rates in IBR patients remained stable in both BC as DCIS patients.

\section{TABLE AND FIGURE LEGEND}

Figure 1. Immediate breast reconstruction and complication rates in invasive breast cancer patients

Figure 2. Immediate breast reconstruction and complication rates in DCIS patients

Table 1. Current Procedural Terminology (CPT) codes for mastectomy and immediate breast reconstruction

Table 2. Overall characteristics invasive breast cancer patients

Table 3. Complication subtype stratified by reconstruction type in invasive breast cancer patients

Table 4. Overall characteristics in DCIS patients

Table 5. Complication subtype stratified by reconstruction type in DCIS patients 


\section{REFERENCES}

1. U.S. Department of Health \& Human Services. Agency for Healthcare Research and Quality (HCUP). NIS description. Information available at: http://www.hcup-us.ahrq.gov/databases.jsp Assessed: June 15th 2015.

2. Smith BD, Smith GL, Hurria A, et al.: Future of cancer incidence in the United States: burdens upon an aging, changing nation. Journal of clinical oncology : official journal of the American Society of Clinical Oncology 2009;27:2758-2765.

3. Shirvani SM, Jiang J, Likhacheva A, et al.: Trends in Local Therapy Utilization and Cost for Early-Stage Breast Cancer in Older Women: Implications for Payment and Policy Reform. International journal of radiation oncology, biology, physics 2016;95:605-616.

4. James R, McCulley SJ, Macmillan RD: Oncoplastic and reconstructive breast surgery in the elderly. The British journal of surgery 2015;102:480-488.

5. Oh DD, Flitcroft K, Brennan ME, Spillane AJ: Patterns and outcomes of breast reconstruction in older women - A systematic review of the literature. European journal of surgical oncology : the journal of the European Society of Surgical Oncology and the British Association of Surgical Oncology 2016;42:604-615.

6. Australian Institute of Health Welfare Cancer Australia. Breast cancer in Australia: an overview. Australian Institute of Health and Welfare; 2012. Available at: http://www.aihw.gov.au/publicationdetail/?id=10737423008 Assessed: September 30, 2016.

7. Breast Cancer Research UK: Breast Cancer Statistics 2014. Available at: http://www.cancerresearchuk.org/health-professional/cancer-statistics/statistics-by-cancer-type/breastcancer Assessed: September 30th, 2016.

8. Song D, Slater K, Papsdorf M, et al.: Autologous Breast Reconstruction in Women Older Than 65 Years Versus Women Younger Than 65 Years: A Multi-Center Analysis. Annals of plastic surgery 2016;76:155-163.

9. Howard-McNatt M, Hughes KS, Schnaper LA, et al.: Breast cancer treatment in older women. Surgical oncology clinics of North America 2005;14:85-102, vi.

10. Hebert-Croteau N, Brisson J, Latreille J, et al.: Compliance with consensus recommendations for the treatment of early stage breast carcinoma in elderly women. Cancer 1999;85:1104-1113.

11. Butz DR, Lapin B, Yao K, et al.: Advanced age is a predictor of 30-day complications after autologous but not implant-based postmastectomy breast reconstruction. Plastic and reconstructive surgery 2015;135:253e-261e.

12. Lee C, Sunu C, Pignone M: Patient-reported outcomes of breast reconstruction after mastectomy: a systematic review. Journal of the American College of Surgeons 2009;209:123-133.

13. Bowman CC, Lennox PA, Clugston PA, Courtemanche DJ: Breast reconstruction in older women: should age be an exclusion criterion? Plastic and reconstructive surgery 2006;118:16-22.

14. Department of Health and Human Services. Centers for Medicare and Medicaid Services. Women's Health and Cancer Rights Act. Available at: http://www.cms.gov/CCIIO/Programs-and-Initiatives/OtherInsurance-Protections/whcra_factsheet.html. Assessed September 142016.

15. Merchant SJ, Goldstein L, Kruper LL: Patterns and Trends in Immediate Postmastectomy Reconstruction in California: Complications and Unscheduled Readmissions. Plastic and reconstructive surgery 2015;136:10e-19e.

16. Lucas DJ, Sabino J, Shriver CD, et al.: Doing more: trends in breast cancer surgery, 2005 to 2011 . The American surgeon 2015;81:74-80.

17. Albornoz CR, Bach PB, Mehrara BJ, et al.: A paradigm shift in U.S. Breast reconstruction: increasing implant rates. Plastic and reconstructive surgery 2013;131:15-23.

18. In H, Jiang $W$, Lipsitz SR, et al.: Variation in the utilization of reconstruction following mastectomy in elderly women. Annals of surgical oncology 2013;20:1872-1879.

19. Lang JE, Summers DE, Cui H, et al.: Trends in post-mastectomy reconstruction: a SEER database analysis. Journal of surgical oncology 2013;108:163-168. 
20. Agarwal S, Pappas L, Neumayer L, Agarwal J: An analysis of immediate postmastectomy breast reconstruction frequency using the surveillance, epidemiology, and end results database. The breast journal 2011;17:352-358.

21. Fischer JP, Tuggle CT, Au A, Kovach SJ: A 30-day risk assessment of mastectomy alone compared to immediate breast reconstruction (IBR). Journal of plastic surgery and hand surgery 2014;48:209-215.

22. Kruper $L, X u X X$, Henderson $K$, et al.: Utilization of mastectomy and reconstruction in the outpatient setting. Annals of surgical oncology 2013;20:828-835.

23. Arrington AK, Voci A, Reparaz L, Fry W: Factors and outcomes associated with surgical treatment options of contralateral breast cancer. American journal of surgery 2014;208:524-530.

24. Miller A, Chandru Kowdley G: Breast reconstruction after mastectomy at an urban community-based program. The American surgeon 2012;78:1281-1284.

25. Wong A, Snook K, Brennan M, et al.: Increasing breast reconstruction rates by offering more women a choice. ANZ journal of surgery 2014;84:31-36.

26. Butler PD, Nelson JA, Fischer JP, et al.: Racial and age disparities persist in immediate breast reconstruction: an updated analysis of 48,564 patients from the 2005 to 2011 American College of Surgeons National Surgery Quality Improvement Program data sets. American journal of surgery 2016;212:96-101.

27. Zhang P, Li CZ, Wu CT, et al.: Comparison of immediate breast reconstruction after mastectomy and mastectomy alone for breast cancer: A meta-analysis. European journal of surgical oncology : the journal of the European Society of Surgical Oncology and the British Association of Surgical Oncology 2016.

28. Jeevan R, Mennie JC, Mohanna PN, et al.: National trends and regional variation in immediate breast reconstruction rates. The British journal of surgery 2016;103:1147-1156.

29. Sabino J, Lucas DJ, Shriver CD, et al.: NSQIP Analysis: Increased Immediate Reconstruction in the Treatment of Breast Cancer. The American surgeon 2016;82:540-545.

30. Hoskin TL, Hieken TJ, Degnim AC, et al.: Use of immediate breast reconstruction and choice for contralateral prophylactic mastectomy. Surgery 2016;159:1199-1209.

31. Habermann EB, Thomsen KM, Hieken TJ, Boughey JC: Impact of availability of immediate breast reconstruction on bilateral mastectomy rates for breast cancer across the United States: data from the nationwide inpatient sample. Annals of surgical oncology 2014;21:3290-3296.

32. Holley DT, Toursarkissian B, Vasconez HC, et al.: The ramifications of immediate reconstruction in the management of breast cancer. The American surgeon 1995;61:60-65.

33. Wilkins EG, Cederna PS, Lowery JC, et al.: Prospective analysis of psychosocial outcomes in breast reconstruction: one-year postoperative results from the Michigan Breast Reconstruction Outcome Study. Plastic and reconstructive surgery 2000;106:1014-1025; discussion 1026-1017.

34. Sullivan SR, Fletcher DR, Isom CD, Isik FF: True incidence of all complications following immediate and delayed breast reconstruction. Plastic and reconstructive surgery 2008;122:19-28.

35. Brennan ME, Spillane AJ: Uptake and predictors of post-mastectomy reconstruction in women with breast malignancy--systematic review. European journal of surgical oncology : the journal of the European Society of Surgical Oncology and the British Association of Surgical Oncology 2013;39:527-541.

36. Hershman DL, Richards CA, Kalinsky K, et al.: Influence of health insurance, hospital factors and physician volume on receipt of immediate post-mastectomy reconstruction in women with invasive and noninvasive breast cancer. Breast cancer research and treatment 2012;136:535-545.

37. Agarwal S, Kidwell KM, Kraft CT, et al.: Defining the relationship between patient decisions to undergo breast reconstruction and contralateral prophylactic mastectomy. Plastic and reconstructive surgery 2015;135:661-670.

38. Naoura I, Mazouni C, Ghanimeh J, et al.: Factors influencing the decision to offer immediate breast reconstruction after mastectomy for ductal carcinoma in situ (DCIS): the Institut Gustave Roussy Breast Cancer Study Group experience. Breast 2013;22:673-675.

39. Aurilio G, Bagnardi V, Nole F, et al.: Outcome of Immediate Breast Reconstruction in Patients With Nonendocrine-Responsive Breast Cancer: A Monoinstitutional Case-Control Study. Clinical breast cancer 2015;15:e237-241. 


\section{Chapter 5}

40. Yang RL, Newman AS, Lin IC, et al.: Trends in immediate breast reconstruction across insurance groups after enactment of breast cancer legislation. Cancer 2013;119:2462-2468.

41. Fischer JP, Wes AM, Tuggle CT, et al.: Risk analysis and stratification of surgical morbidity after immediate breast reconstruction. Journal of the American College of Surgeons 2013;217:780-787.

42. Santosa KB, Qi J, Kim HM, et al.: Effect of Patient Age on Outcomes in Breast Reconstruction: Results from a Multicenter Prospective Study. Journal of the American College of Surgeons 2016.

43. Pope D, Ramesh H, Gennari R, et al.: Pre-operative assessment of cancer in the elderly (PACE): a comprehensive assessment of underlying characteristics of elderly cancer patients prior to elective surgery. Surgical oncology 2006;15:189-197.

44. Jagsi R, Jiang J, Momoh AO, et al.: Complications After Mastectomy and Immediate Breast Reconstruction for Breast Cancer: A Claims-Based Analysis. Annals of surgery 2016;263:219-227. 
Chapter

\section{The National Surgical Quality Improvement Program 30-Day Challenge: Microsurgical Breast Reconstruction Outcomes Reporting Reliability}

\section{Austin D. Chen}

Parisa Kamali

Amnol S. Chattha

Alexandra Bucknor

Justin B. Cohen

Patrick P. Bletsis

Renata Flecha-Hirsch

Adam M. Tobias

Bernard T. Lee

Samuel J. Lin 


\section{ABSTRACT}

\section{Introduction}

The aim is to assess reliability of the American College of Surgeons National Surgical Quality Improvement Program (ACS-NSQIP) 30-day perioperative outcomes and complications for immediate, free-tissue transfer breast reconstruction (IFTBR) by direct comparisons to our 30-day and overall institutional data, and assessing those that occur after 30 days.

\section{Methods}

Data were retrieved for consecutive IFTBR patients from a single-institution database (2010-2015) and the ACS-NSQIP (2011-2014). Multiple logistic regressions were performed to compare adjusted outcomes between the two datasets.

\section{Results}

For institutional vs. ACS-NQSIP outcomes, there were no significant differences in surgical site infection (SSI) (30-day: $3.6 \%$ vs. $4.1 \%, p=0.818$; overall: $5.3 \%$ vs. $4.1 \%$, $p=0.198$ ), wound disruption (WD) (30-day: $1.3 \%$ vs. $1.5 \%, p=0.526$; overall: $2.3 \%$ vs. $1.5 \%, p=0.560$ ), or unplanned readmission (URA) (30-day: $2.3 \%$ vs. $3.3 \%, p=0.714$; overall: $4.6 \%$ vs. $3.3 \%, p=0.061)$. However, the ACS-NSQIP reported a significantly higher unplanned reoperation (URO) rate (30-day: $3.6 \%$ vs. $9.5 \%, p<0.001$; overall: $5.3 \%$ vs. $9.5 \%, p=0.025)$. Institutional complications consisted of $5.3 \% \mathrm{SSI}, 2.3 \% \mathrm{WD}, 5.3 \%$ URO, and $4.6 \%$ URA, of which $25.0 \%$ SSI, 28.6\% WD, $12.5 \%$ URO, and $7.1 \%$ URA occurred at $30-60$ days, and $6.3 \% \mathrm{SSI}, 14.3 \% \mathrm{WD}, 18.8 \%$ URO, and $42.9 \%$ URA occurred after 60 days.

\section{Conclusion}

For immediate free tissue breast reconstruction, the ACS-NSQIP may be reliable for monitoring and comparing surgical site infection, wound disruption, unplanned reoperation, and unplanned readmission rates. However, clinicians may find it useful to understand limitations of the ACS-NSQIP for complications and risk factors, as it may underreport complications occurring beyond 30 days. 


\section{INTRODUCTION}

The use of large-volume databases in surgical outcomes research has grown substantially over the last decade, with surgeon, hospital, and regional-level outcomes increasingly being evaluated using clinical outcomes and measures of resource utilization. $^{1-29}$ Large-volume databases can be broadly categorized as either administrative or clinical. These databases offer unique opportunities to study largescale patterns of care, variation in practice, and outcomes following surgical intervention. Studies based on national registries and other administrative datasets have made significant contributions to the field of breast cancer surgery. ${ }^{1-24}$ In recent years, research derived from these large-data registries has played an increasing role in the development of clinical guidelines and health policy within the field of breast cancer treatment. ${ }^{1-24}$ One such validated large-volume clinical database is the American College of Surgeons National Surgical Quality Improvement Program (ACS-NSQIP) database. ${ }^{30}$ Its validity hinges on the number of involved hospitals, accurate recording of key patient variables, and methodical follow-up to 30 days postoperatively. ${ }^{31}$ This database has helped in multiple areas within plastic surgery, including the recognition of risk factors for venous thromboembolism in breast reconstruction, the complications associated with contralateral prophylactic mastectomy, and the impact of surgical resident involvement in breast reduction. ${ }^{15,17,23}$

Despite the versatility of the ACS-NSQIP database, several studies in multiple disciplines have questioned its validity, primarily in terms of the coding process and built-in variables. ${ }^{32-37}$ Within plastic surgery, the ACS-NQIP Surgical Risk Calculator has been assessed and deemed inaccurate for predicting risk factors for complications after breast reconstruction. ${ }^{32,33}$ Furthermore, the reported incidence of complications as defined by variables in the database has come under question, and by extension, the validity of the risk factors identified using the database. ${ }^{34-37}$ These issues primarily stem from the question of whether 30 days' follow-up is sufficient to capture the full scope of complications. Recently published literature investigated the rate of complications occurring beyond 30 days in patients undergoing alloplastic breast reconstruction. ${ }^{34-36}$

Incidence and timing of complications were investigated by Luce et al. for tissue expander explantation and by Cohen et al. and Sinha et al. for infections in implants and tissue expanders. ${ }^{34-36}$ In autologous breast reconstruction, Duraes et al. reported on the incidence of institutional late complications occurring after 30 days in abdominal-based free flap procedures. ${ }^{37}$ However, to date, there is a lack of literature on the presence and timing of complications in ACS-NSQIP for autologous breast reconstruction. To the best of our knowledge, no previous studies have directly compared institutional data with ACS-NSQIP to assess whether the national database is valid and applicable to institutional practice, within both the 30-day perioperative period or after 30-days.

Given this lack of knowledge, the aim of this study is to evaluate the reliability of the complications captured by the ACS-NSQIP within its early 30-day window for immediate 
free tissue breast reconstruction (IFTBR). This will be conducted by direct comparison of the outcomes reported in the ACS-NSQIP to both our 30-day and overall (complications that occur within 30 days and onwards) institutional data, to determine whether the database is reliable for complication monitoring and comparison studies. A secondary aim is to report the incidence and timing breakdown of late complications after autologous breast reconstruction, to determine whether ACS-NSQIP is reliable for a true overall complication profile and risk factor calculation studies.

\section{METHODS}

Institutional review board approval was obtained. Data were collected from patient records within a single institution from 2010-2015 with a minimum follow up of one year, and ACS-NSQIP data were retrieved for the years 2011-2014 using Current Procedural Terminology (CPT) codes (See Table, Supplementary Digital Content 1, Current Procedural Terminology (CPT) mastectomy and breast reconstruction codes). We extracted data from the respective time periods to account for as many possible data points for the variables of interest. Our inclusion criteria consisted of female patients over the age of 18 who underwent IFTBR following mastectomy. We excluded patients who underwent combined free tissue reconstruction with other autologous or alloplastic techniques. In ACS-NSQIP, a patient was considered to have undergone IFTBR if concurrent mastectomy and reconstruction CPT codes were registered.

Patient characteristics of interest were restricted to those recorded in both the institutional and ACS-NSQIP databases, to enable direct comparison. These included age, body mass index (BMI), smoking, diabetes, hypertension, coagulopathy, steroid use, number of comorbidities, mastectomy type, operation time (OT) in minutes, and length of stay (LOS) in days. Bilateral mastectomy was determined based on presence of two CPT codes for mastectomy. Outcomes of interest were surgical site infection, wound disruption, unplanned reoperation, unplanned readmission, and the specific causes of unplanned reoperation or readmission. Prior to 2011, the ACS-NSQIP data did not include the cause of unplanned reoperation and unplanned readmission variables. As such, unplanned reoperation and readmission data were extracted from ACS-NSQIP 2012-2014. Institutional complications were only recorded if they were related to the index IFTBR procedure and fit the ACS-NSQIP definitions for surgical site infection, wound disruption, unplanned reoperation, and unplanned readmission.

Unplanned reoperation was classified into categories based on correlating ACSNSQIP variables for the root cause, consisting of complications pertaining to the flap itself, surgical site infection, wound site disruption, hemorrhage, hematoma, and seroma. Unplanned readmission was classified into categories based on correlating ACSNSQIP variables for the root cause, consisting of complications pertaining to the flap itself, surgical site infection, wound site disruption, hematoma, seroma, and 
postoperative pain. These were extracted from the ACS-NSQIP (2012-2014) using the inbuilt reason for unplanned reoperation variable and International Classification of Diseases, Ninth Revision, Clinical Modification (ICD-9-CM) codes (See Table, Supplementary Digital Content 2, International Classification of Diseases, Ninth Revision, Clinical Modification (ICD-9-CM) cause of unplanned reoperation or readmission codes).

Institutional outcomes in the 30-day window and overall, including both 30-day complications and those occurring after 30 days, were each independently compared to ACS-NSQIP outcomes to assess reliability of the database. Late complications occurring after 30 days are representative of the number of complications potentially missed by ACS-NSQIP. Institutional outcomes were sub-grouped into those that occurred within the 30-day window (early), those that occurred after 30 days (late), and the overall (early and late) complication incidence. In addition, complications that occurred after 30 days were further sub-categorized into 60-day and 60-day+ groups.

\section{Statistical analysis}

Data were compared using Pearson's $\chi 2$ or Fisher's exact test tests and Wilcoxon-MannWhitney for categorical and nonparametric continuous variables, respectively. To account and adjust for potential confounders when analyzing outcomes of interest, logistic regression models were used to assess patient outcomes of surgical site infection, wound disruption, unplanned reoperation, unplanned readmission, and causes of unplanned reoperation or readmission. Statistical analysis was performed using SPSS Version 22 (IBM, Armonk, NY). For all analysis, a value of $\mathrm{p}<0.05$ was considered statistically significant.

\section{RESULTS}

\section{Patient Characteristics}

During the study period, a total of 2,402 patients were admitted for IFTBR, with 304 (12.6\%) patients from our institution and 2,098 (87.3\%) patients from the ACS-NSQIP (2011-2014) (Table 1). Patients were well matched for most patient characteristics. No significant differences were observed in age $(p=0.315)$, diabetes $(p=0.680)$, coagulopathy $(p=0.675)$, steroid use $(p=0.613)$, number of comorbidities $(p=0.350)$, LOS $(p=0.274)$, and proportion of radical mastectomy $(p=0.114)$. Although the average patient in both was classified by BMI as 'obese', patients in our institution had a significantly lower BMI (28.2 \pm 5.6 vs. $\left.29.9 \pm 5.9 \mathrm{~kg} / \mathrm{m}^{2}, \mathrm{p}<0.001\right)$, longer OT (702.4 \pm 166.9 vs. $524.0 \pm 182.3$ minutes, $\mathrm{p}<0.001$ ), and underwent more bilateral $(55.9 \%$ vs. $40.2 \%, p<0.001$ ) and total simple mastectomies (96.7\% vs. $82.3 \%, p<0.001$ ) when compared to the ACS-NSQIP database. However, fewer patients in our institutional group were smokers (3.6\% vs. $8.9 \%, p=0.002)$, had hypertension $(18.4 \%$ vs. $24.6 \%$, 
$\mathrm{p}=0.018)$, or underwent modified radical mastectomy $(1.6 \%$ vs. $14.0 \%, p<0.001)$ compared to the ACS-NSQIP database. Patients in our institutional database underwent deep inferior epigastric perforator (DIEP) (96.2\%), superior gluteal artery perforator (SGAP) (3.3\%), and free transverse rectus abdominis myocutaneous (free TRAM) (0.2\%) flap reconstructions, whereas this breakdown was not able to be assessed in the ACSNSQIP database.

Table 1. Institutional vs. the ACS-NSQIP patient characteristics.

\begin{tabular}{|c|c|c|c|c|c|}
\hline \multirow[b]{2}{*}{ Patient Characteristics } & \multicolumn{2}{|c|}{ Institutional } & \multicolumn{2}{|c|}{ ACS-NSQIP } & \multirow[b]{2}{*}{$P$} \\
\hline & $\mathbf{n}$ & $(\%)$ & $\mathbf{n}$ & $(\%)$ & \\
\hline Total & 304 & $(12.6)$ & 2,098 & $(87.3)$ & \\
\hline Age $(y)$ & 51.19 & \pm 9.14 & 50.55 & \pm 9.16 & 0.315 \\
\hline $\mathrm{BMI}\left(\mathrm{kg} / \mathrm{m}^{2}\right)$ & 28.2 & \pm 5.6 & 29.9 & \pm 5.9 & $<0.001$ \\
\hline Smoking & 11 & $(3.6)$ & 187 & $(8.9)$ & 0.002 \\
\hline Diabetes & 13 & $(4.3)$ & 101 & $(4.8)$ & 0.680 \\
\hline Hypertension & 56 & (18.4) & 516 & $(24.6)$ & 0.018 \\
\hline Coagulopathy & 2 & $(0.7)$ & 11 & $(0.5)$ & 0.675 \\
\hline Steroid use & 3 & $(1.0)$ & 32 & $(1.5)$ & 0.613 \\
\hline No. comorbidities & & & & & 0.350 \\
\hline 0 & 233 & $(76.6)$ & 1,531 & $(73.0)$ & 0.176 \\
\hline 1 & 63 & $(20.7)$ & 498 & $(23.7)$ & 0.246 \\
\hline$\geq 2$ & 8 & $(2.6)$ & 68 & $(3.2)$ & 0.570 \\
\hline \multicolumn{6}{|l|}{ Mastectomy } \\
\hline Bilateral & 170 & $(55.9)$ & 844 & $(40.2)$ & $<0.001$ \\
\hline Simple & 294 & $(96.7)$ & 1,726 & $(82.3)$ & $<0.001$ \\
\hline Modified radical & 5 & $(1.6)$ & 293 & $(14.0)$ & $<0.001$ \\
\hline Radical & 2 & $(0.7)$ & 44 & $(2.1)$ & 0.114 \\
\hline OT $(\min )$ & 702.4 & \pm 166.9 & 524.0 & \pm 182.3 & $<0.001$ \\
\hline Length of stay (d) & 4.33 & \pm 1.41 & 4.64 & \pm 8.42 & 0.274 \\
\hline
\end{tabular}

Bold type signifies $p$-value has reached statistical significance.

\section{Patient Outcomes (Institution vs. ACS-NSQIP)}

Table 2 summarizes the adjusted patient outcomes for 30-day and overall institutional vs. the ACS-NSQIP, respectively. No significant differences were seen when comparing either institutional 30-day or overall complications to ACS-NSQIP outcomes for surgical site infection (30-day: $3.6 \%$ vs. $4.1 \%, p=0.818$; overall: $5.3 \%$ vs. $4.1 \%, p=0.198$ ), wound disruption (30-day: $1.3 \%$ vs. $1.5 \%, p=0.526$; overall: $2.3 \%$ vs. $1.5 \%, p=0.560$ ), and unplanned readmission (30-day: $2.3 \%$ vs. $3.3 \%, p=0.714$; overall: $4.6 \%$ vs. $3.3 \%$, $\mathrm{p}=0.061$ ). However, there were significantly lower unplanned reoperation rates in our institutional data compared with ACS-NSQIP data (30-day: 3.6\% vs. 9.5\%, $p<0.001$; overall: $5.3 \%$ vs. $9.5 \%, p=0.025)$. Institutionally, five (31.3\%) of 16 unplanned reoperations and three (21.4\%) of 14 unplanned readmissions were independent, with an overlap between unplanned reoperations and readmissions in the remaining 11 cases. In the ACS-NSQIP, 136 (78.6\%) of 173 unplanned reoperations and 23 (38.3\%) of 
60 unplanned readmissions were independent, with an overlap between unplanned reoperations and readmissions in the remaining 37 cases. The discrepancy may be due to some reoperations not necessarily necessitating a readmission, or vice versa, due to the ACS-NSQIP definition of an unplanned readmission being for an "inpatient" stay or an unplanned reoperation being performed within the same index inpatient stay.

Table 2. Institutional (total and $\leq 30$ days) vs. the ACS-NSQIP outcomes.

\begin{tabular}{|c|c|c|c|c|c|}
\hline \multirow[b]{2}{*}{ Patient Outcomes } & \multicolumn{2}{|c|}{ Institutional } & \multicolumn{2}{|c|}{ ACS-NSQIP } & \multirow[b]{2}{*}{$P$} \\
\hline & n & $(\%)$ & $\mathbf{n}$ & $(\%)$ & \\
\hline \multicolumn{6}{|l|}{ SSI } \\
\hline Total & 16 & $(5.3)$ & 86 & $(4.1)$ & 0.198 \\
\hline $30-d$ & 11 & $(3.6)$ & 86 & $(4.1)$ & 0.818 \\
\hline \multicolumn{6}{|l|}{ WD } \\
\hline Total & 7 & $(2.3)$ & 31 & $(1.5)$ & 0.560 \\
\hline $30-d$ & 4 & (1.3) & 31 & $(1.5)$ & 0.526 \\
\hline \multicolumn{6}{|l|}{ URO } \\
\hline Total & 16 & $(5.3)$ & $173 *$ & $(9.5)$ & 0.025 \\
\hline $30-d$ & 11 & $(3.6)$ & $173 *$ & $(9.5)$ & $<0.001$ \\
\hline \multicolumn{6}{|l|}{ URA } \\
\hline Total & 14 & $(4.6)$ & $60 \dagger$ & $(3.3)$ & 0.061 \\
\hline $30-d$ & 7 & $(2.3)$ & $60 \dagger$ & $(3.3)$ & 0.714 \\
\hline
\end{tabular}

*This value was determined after excluding any UROs not categorizable for comparison. It was extracted from ACS-NSQIP (2012-2014).

$\uparrow$ This value was determined after excluding any URAs not categorizable for comparison. It was extracted from ACS-NSQIP (2012-2014).

Bold type signifies $p$-value has reached statistical significance.

\section{Unplanned Reoperation (Institutional vs. ACS-NSQIP)}

Reasons for unplanned reoperation are listed in Table 3. There were significantly fewer unplanned reoperations reported in our institutional database compared to the ACSNSQIP after selecting for specific complications (30-day: 3.6\% vs. 9.5\%, $p<0.001$; overall: $5.3 \%$ vs. $9.5 \%, p=0.025)$. Compared with institutional data, there were a greater number of hematomas requiring unplanned reoperation in the ACS-NSQIP database (30-day: $1.0 \%$ vs. $4.1 \%, p=0.009$; overall: $1.0 \%$ vs. $4.1 \%, p=0.009$ ). 
Table 3. Institutional (total and $\leq 30$ days) vs. the ACS-NSQIP (2012-2014) categorized unplanned reoperations.

\begin{tabular}{|c|c|c|c|c|c|}
\hline \multirow[b]{2}{*}{ Patient Outcomes } & \multicolumn{2}{|c|}{ Institutional } & \multicolumn{2}{|c|}{ ACS-NSQIP } & \multirow[b]{2}{*}{$P$} \\
\hline & $\mathbf{n}$ & $(\%)$ & $\mathbf{n}$ & $(\%)$ & \\
\hline \multicolumn{6}{|l|}{ URO } \\
\hline Total & 16 & $(5.3)$ & $173 *$ & $(9.5)$ & 0.025 \\
\hline $30-\mathrm{d}$ & 11 & $(3.6)$ & $173^{*}$ & $(9.5)$ & $<0.001$ \\
\hline \multicolumn{6}{|l|}{ Flap complication } \\
\hline Total & 5 & $(1.6)$ & 40 & $(2.2)$ & 0.675 \\
\hline $30-d$ & 5 & $(1.6)$ & 40 & $(2.2)$ & 0.675 \\
\hline \multicolumn{6}{|l|}{ Infection } \\
\hline Total & 1 & $(0.3)$ & 15 & $(0.8)$ & 0.454 \\
\hline $30-d$ & 0 & $(0.0)$ & 15 & $(0.8)$ & 0.994 \\
\hline \multicolumn{6}{|l|}{ WD } \\
\hline Total & 6 & $(2.0)$ & 41 & $(2.2)$ & 0.887 \\
\hline $30-d$ & 3 & $(1.0)$ & 41 & $(2.2)$ & 0.297 \\
\hline \multicolumn{6}{|l|}{ Hemorrhage } \\
\hline Total & 1 & $(0.3)$ & 5 & $(0.3)$ & 0.865 \\
\hline $30-d$ & 1 & $(0.3)$ & 5 & $(0.3)$ & 0.865 \\
\hline \multicolumn{6}{|l|}{ Hematoma } \\
\hline Total & 3 & $(1.0)$ & 75 & (4.1) & 0.009 \\
\hline $30-\mathrm{d}$ & 3 & $(1.0)$ & 75 & (4.1) & 0.009 \\
\hline \multicolumn{6}{|l|}{ Seroma } \\
\hline Total & 3 & $(1.0)$ & 6 & $(0.3)$ & 0.118 \\
\hline $30-d$ & 2 & $(0.7)$ & 6 & $(0.3)$ & 0.393 \\
\hline
\end{tabular}

*This value was determined after excluding any UROs not categorizable for comparison.

Bold type signifies $p$-value has reached statistical significance.

Unplanned Readmission (Institutional vs. ACS-NSQIP)

Reasons for unplanned readmission are listed in Table 4. There were no significant differences in unplanned readmissions reported in our institutional database compared to the ACS-NSQIP after selecting for specific complications (30-day: $2.3 \%$ vs. $3.3 \%$, $p=0.714$; overall: $4.6 \%$ vs. $3.3 \%, p=0.061$ ). The number of infections requiring unplanned readmission occurring after 30 days was substantial enough to show a significantly higher rate in overall institutional vs. the ACS-NSQIP data (30-day: 1.6\% vs. $1.8 \%, p=0.850$; overall: $3.3 \%$ vs. $1.8 \%, p=0.031$ ). 
Table 4. Institutional (total and $\leq 30$ days) vs. the ACS-NSQIP (2012-2014) categorized unplanned readmissions.

\begin{tabular}{|c|c|c|c|c|c|}
\hline \multirow[b]{2}{*}{ Patient Outcomes } & \multicolumn{2}{|c|}{ Institutional } & \multicolumn{2}{|c|}{ ACS-NSQIP } & \multirow[b]{2}{*}{$P$} \\
\hline & $\mathbf{n}$ & $(\%)$ & $\mathbf{n}$ & $(\%)$ & \\
\hline \multicolumn{6}{|l|}{ URA } \\
\hline Total & 14 & $(4.6)$ & $60 *$ & $(3.3)$ & 0.061 \\
\hline $30-d$ & 7 & $(2.3)$ & $60 *$ & $(3.3)$ & 0.714 \\
\hline \multicolumn{6}{|l|}{ Flap complication } \\
\hline Total & 1 & $(0.3)$ & 6 & $(0.6)$ & 0.526 \\
\hline $30-d$ & 1 & $(0.3)$ & 6 & $(0.6)$ & 0.526 \\
\hline \multicolumn{6}{|l|}{ Infection } \\
\hline Total & 10 & $(3.3)$ & 33 & $(1.8)$ & 0.031 \\
\hline $30-d$ & 5 & $(1.6)$ & 33 & $(1.8)$ & 0.850 \\
\hline \multicolumn{6}{|l|}{ WD } \\
\hline Total & 1 & $(0.3)$ & 15 & $(0.8)$ & 0.631 \\
\hline $30-d$ & 0 & $(0.0)$ & 15 & $(0.8)$ & 0.994 \\
\hline \multicolumn{6}{|l|}{ Hematoma } \\
\hline Total & 1 & $(0.3)$ & 4 & $(0.2)$ & 0.714 \\
\hline $30-d$ & 1 & $(0.3)$ & 4 & $(0.2)$ & 0.714 \\
\hline \multicolumn{6}{|l|}{ Seroma } \\
\hline Total & 1 & $(0.3)$ & 1 & $(0.1)$ & 0.204 \\
\hline $30-\mathrm{d}$ & 0 & $(0.0)$ & 1 & $(0.1)$ & 0.995 \\
\hline \multicolumn{6}{|l|}{ Postoperative pain } \\
\hline Total & 1 & $(0.3)$ & 2 & $(0.1)$ & 0.806 \\
\hline $30-d$ & 1 & $(0.3)$ & 2 & $(0.1)$ & 0.806 \\
\hline
\end{tabular}

*This value was determined after excluding any UROs not categorizable for comparison.

Bold type signifies $p$-value has reached statistical significance.

\section{Early vs. Late Complications (Institution)}

In our institutional database, the complication profile consisted of $5.3 \%$ surgical site infections, 2.3\% wound disruptions, 5.3\% unplanned reoperations, and $4.6 \%$ unplanned readmissions. Table 5 summarizes the percentage of these complications occurring after the 30-days. When observing what percentage of total complications were late, we found that $31.3 \%$ ( $25.0 \%$ by $30-60$ days, $6.3 \%$ after 60 days) of surgical site infections, $42.9 \%$ of wound disruptions ( $28.6 \%$ by $30-60$ days, $14.3 \%$ after 60 days), $31.3 \%$ of unplanned reoperations ( $12.5 \%$ by $30-60$ days, $18.8 \%$ after 60 days), and $50.0 \%$ of unplanned readmissions ( $7.1 \%$ by $30-60$ days, $42.9 \%$ after 60 days) occurred after 30 days. 
Table 5. Timing of complications breakdown (Institutional).

\begin{tabular}{|c|c|c|c|c|c|c|c|c|}
\hline Patient Outcomes & \multicolumn{2}{|c|}{ Overall } & \multicolumn{2}{|c|}{$30-d$} & \multicolumn{2}{|c|}{$60-\mathrm{d}$} & \multicolumn{2}{|c|}{$60-d+$} \\
\hline SSI & 16 & $(100.0)$ & 11 & $(68.8)$ & 4 & $(25.0)$ & 1 & $(6.3)$ \\
\hline URO & 16 & $(100.0)$ & 11 & $(68.8)$ & 2 & (12.5) & 3 & (18.8) \\
\hline Flap complication & 5 & $(100.0)$ & 5 & $(100.0)$ & 0 & $(0.0)$ & 0 & $(0.0)$ \\
\hline Infection & 1 & $(100.0)$ & 0 & $(0.0)$ & 1 & $(100.0)$ & 0 & $(0.0)$ \\
\hline WD & 6 & $(100.0)$ & 3 & $(50.0)$ & 1 & (16.7) & 2 & (33.3) \\
\hline Seroma & 3 & $(100.0)$ & 2 & $(66.7)$ & 0 & $(0.0)$ & 1 & (33.3) \\
\hline URA & 14 & $(100.0)$ & 7 & $(50.0)$ & 1 & (7.1) & 6 & $(42.9)$ \\
\hline Flap complication & 1 & $(100.0)$ & 1 & $(100.0)$ & 0 & $(0.0)$ & 0 & $(0.0)$ \\
\hline Infection & 10 & $(100.0)$ & 5 & $(50.0)$ & 1 & $(10.0)$ & 4 & $(40.0)$ \\
\hline WD & 1 & $(100.0)$ & 0 & $(0.0)$ & 0 & $(0.0)$ & 1 & $(100.0)$ \\
\hline Hematoma & 1 & $(100.0)$ & 1 & $(100.0)$ & 0 & $(0.0)$ & 0 & $(0.0)$ \\
\hline Seroma & 1 & $(100.0)$ & 0 & $(0.0)$ & 0 & $(0.0)$ & 1 & $(100.0)$ \\
\hline
\end{tabular}

\section{DISCUSSION}

Large clinical databases such as the ACS-NSQIP serve as a unique platform for retrospective clinical studies, providing large patient populations suitable for studying outcomes and variations in treatment. Within the field of breast reconstruction, largevolume databases are being increasingly utilized. ${ }^{1-20}$ Studies based on clinical databases have made significant contributions to the field of plastic surgery with development of clinical guidelines and health policy. It is important for clinicians and researchers to understand the strengths and weaknesses of these databases to enable appropriate data interpretation. ${ }^{19}$

The current study aims to assess the validity of the ACS-NSQIP database for IFTBR by comparing its reported incidence of complications to those reported in a reasonably high-volume academic center. Our results show that ACS-NSQIP may accurately represent the incidence of both 30-day and overall complications for surgical site infection, wound disruption, and unplanned readmission, and as such be reliable for complication monitoring and comparison studies. However, it did not accurately capture overall unplanned readmissions due to infection. The ACS-NSQIP also reported a significantly higher rate of unplanned reoperation than that found in our institutional data, which was attributable to the higher rate of unplanned reoperation for hematoma. While there were no significant differences between overall vs. ACS-NSQIP complication rates, we found that a large percentage of surgical site infections, wound disruptions, unplanned reoperations, and unplanned readmissions occur after the 30day window, suggesting that ACS-NSQIP may underreport complications. As such, it may not be reliable for studies evaluating true overall complication profiles or risk factor calculation.

Unplanned reoperation rates were one important difference between institutional and ACS-NSQIP data, with ACS-NSQIP unplanned reoperation rates being significantly higher; this persisted when selecting for IFTBR-specific complications. It may be that variation exists in institutional operative practices and decision-making protocols for 
reoperation, explaining our findings. A study on autologous breast reconstruction conducted using the National Inpatient Sample Healthcare Cost and Utilization Project has shown that high-volume centers have lower complications, with the volumeoutcome relationship being more strongly associated with surgery-specific rather than systematic complications. ${ }^{38}$ The literature for microsurgical breast reconstruction has reported lower flap loss rates and improved salvage rates associated with tissue oximetry, with decreased rate of re-exploration over time per 100 flaps operated on. ${ }^{39-}$ ${ }^{42}$ The use of more than one venous outflow vessel may also prevent unplanned reoperation. ${ }^{43}$

When reviewing the causes for unplanned reoperation, hematoma appeared to contribute to the higher rates of unplanned reoperation in ACS-NSQIP, compared with institutional data. The lower rates found in our institutional data are supported by a previous review article outlining unplanned reoperation for hematomas in microvascular free tissue transfers, noting rates ranging from $0.2 \%-9 \% .{ }^{44}$ Halle et al. reported a $13 \%$ incidence of reoperations for hematomas in breast free flaps, highlighting the potential risk of antithrombotic use and importance of using drains. ${ }^{45} \mathrm{~A}$ study assessing risk factors for hematoma formation in 883 patients who underwent mastectomy and immediate reconstruction found no measurable preoperative, operative or oncologic risk factors, citing that meticulous hemostasis may be one of the factors. ${ }^{46}$

It is important to note that a large percentage of surgical site infections, wound disruptions, unplanned reoperations, and unplanned readmissions occurred after 30 days, highlighting the possibility of an underreported complication rate in ACS-NSQIP. More specifically, the majority of surgical site infections and wound disruptions occurred within 60 days, whereas the majority of unplanned reoperations and unplanned readmissions occurred after the 60-day period. This could be due to several temporal factors, including time taken for clinical deterioration sufficient to warrant unplanned reoperation or unplanned readmission, or time required to arrange for patient hospital admission. All unplanned reoperations and readmissions for flap complication, hemorrhage, or hematoma occurred within 30 days. The majority of unplanned reoperations and readmissions for infection, seroma, and wound disruption occurred after 30 days. It may be that the later reoperations and readmissions occurred as a result of managing conservatively at first for these complications. Furthermore, late management of seromas may be linked to the pathophysiology of seroma formation, which requires time for fluid collection. A study on abdominal-based free tissue breast reconstruction complications by Duraes et al. also found that a large percentage of complications were late and inferred that the ACS-NSQIP 30-day follow up may not be sufficient. ${ }^{37}$ The percentages of early 30-day and late infection complications found in our data differed from those reported by Dureas et al. (early: $68.8 \%$ vs. $89.0 \%$, late: $31.3 \%$ vs. $11.0 \%) .{ }^{37}$ This finding may be due to the differing surgical teams, surgical technique, patient characteristics, or type of reconstruction. It may be prudent to 
extend the ACS-NSQIP follow up period to up to three months, with further studies evaluating the optimum follow up time for maximum capture of complications.

Studies have also reported a large percentage of late complications within alloplastic breast reconstruction, with Luce et al. reporting that $65 \%$ of tissue expanders destined for loss were still in situ at 30 days, Sinha et al. reporting that $47 \%$ to $71 \%$ of surgical site infections were late (>30 days), and Cohen et al. reporting that $50 \%$ of infections were late (>30 days). ${ }^{34-36}$ Compared to these studies of alloplastic reconstruction, we found a lower rate of late complications. Similar findings were described by Mioton et al. in their report of 30-day complications, describing greater differences in autologous vs. implant complications (infection: $5.46 \%$ vs. $3.45 \%$, p<0.001; wound disruption: $1.24 \%$ vs. $0.44 \%$, $\mathrm{p}<0.001$; reoperation: $9.59 \%$ vs. $6.76 \%, p<0.001) .{ }^{47}$ It may be interesting to assess the risk factors for early and late complications in autologous compared with implant reconstruction.

We acknowledge the limitations of our study. Retrospective chart reviews are at risk of human error in the data collection process. We were unable to assess certain parameters due to the presence of in-built variables in ACS-NSQIP, including radiotherapy and chemotherapy. For future reference, ACS-NSQIP may look to introduce these variables. We were also unable to sub-categorize specific IFTBR procedures for comparison, such as DIEP, SGAP, or free TRAM, due to limitations of CPT coding. The scope of the study was also limited to complications defined in ACS-NSQIP. As such, we could not analyze important outcomes such as donor vs. recipient complications, mastectomy skin necrosis, fat necrosis, or abdominal hernia development. The inclusion of these variables may further surgical clinical outcomes' research, with more targeted, inclusive data. Due to the single-center study comparison, and the uniqueness of the protocol at our high-volume center, this may have led to differences in the comparison of our outcomes versus those hospitals captured by the ACS-NSQIP, who may perform a lower number of free tissue breast reconstructions. Despite these, we believe that our study makes important contributions to the current literature, and to our knowledge, this is the first study to report a head-to-head comparison of outcomes between a single institution and a national database.

\section{CONCLUSION}

For complication monitoring and comparison studies, the ACS-NSQIP may reliably represent the general scope of surgical site infections, wound disruptions, unplanned reoperations, and unplanned readmissions in institutional data for immediate free tissue breast reconstruction; however, it may not generally capture unplanned readmissions for infection occurring after 30 days. There was also a significantly higher rate of unplanned reoperations for ACS-NSQIP, which was due to the differences in unplanned reoperations 
for hematoma. A large percentage of complications in our institutional database occurred after 30 days, and as such, clinicians and researchers should continue to exercise caution when reporting overall complication rates or assessing risk factors for future guidelines. An extension of the follow-up beyond 30 days should be considered.

\section{TABLE LEGEND}

Table 1. Institutional vs. the ACS-NSQIP patient characteristics

Table 2. Institutional (total and $\leq 30$ days) vs. the ACS-NSQIP outcomes

Table 3. Institutional (total and $\leq 30$ days) vs. the ACS-NSQIP (2012-2014) categorized unplanned reoperations

Table 4. Institutional (total and $\leq 30$ days) vs. the ACS-NSQIP (2012-2014) categorized unplanned readmissions

Table 5. Timing of complications breakdown (Institutional)

\section{SUPPLEMENTARY DIGITAL CONTENT}

Table, Supplementary Digital Content 1. Current Procedural Terminology (CPT) mastectomy and breast reconstruction codes

Table, Supplementary Digital Content 2. International Classification of Diseases, Ninth Revision, Clinical Modification (ICD-9-CM) cause of unplanned reoperation and readmission codes 


\section{SUPPLEMENTARY DIGITAL CONTENT}

Table, Supplementary Digital Content 1. Current Procedural Terminology (CPT) mastectomy and breast reconstruction codes.

\begin{tabular}{|c|c|}
\hline Procedural description & CPT Code \\
\hline & Inclusion criteria \\
\hline Mastectomy, simple, complete & 19303 \\
\hline Mastectomy, subcutaneous & 19304 \\
\hline $\begin{array}{l}\text { Mastectomy, radical, including pectoral muscles, } \\
\text { axillary lymph nodes }\end{array}$ & 19305 \\
\hline $\begin{array}{l}\text { Mastectomy, radical, including pectoral muscles, } \\
\text { axillary and internal mammary lymph nodes (urban } \\
\text { type operation) }\end{array}$ & 19306 \\
\hline \multicolumn{2}{|c|}{$\begin{array}{l}\text { Mastectomy, modified radical, including axillary lymph } 19307 \\
\text { nodes, with or without pectoralis minor muscle, but } \\
\text { excluding pectoralis major muscle }\end{array}$} \\
\hline \multirow[t]{2}{*}{ Breast reconstruction with free flap } & 19364 \\
\hline & Exclusion criteria \\
\hline $\begin{array}{l}\text { Mastectomy, partial (eg, lumpectomy, tylectomy, } \\
\text { quadrantectomy, segmentectomy); with/without } \\
\text { axillary lymphadenectomy }\end{array}$ & 19301, 19302 \\
\hline \multirow{2}{*}{$\begin{array}{l}\text { Immediate insertion of breast prosthesis following } \\
\text { mastopexy, mastectomy or in reconstruction }\end{array}$} & 19340 \\
\hline & Exclusion criteria \\
\hline $\begin{array}{l}\text { Delayed insertion of breast prosthesis following } \\
\text { mastoplexy, mastectomy, or in reconstruction }\end{array}$ & 19342 \\
\hline $\begin{array}{l}\text { Breast reconstruction, immediate or delayed, with } \\
\text { tissue }\end{array}$ & 19357 \\
\hline $\begin{array}{l}\text { Breast reconstruction with latissimus dorsi flap, } \\
\text { without prosthetic implant }\end{array}$ & 19361 \\
\hline $\begin{array}{l}\text { Breast reconstruction with transverse rectus } \\
\text { abdominis myocutaneous flap (TRAM), single pedicle, } \\
\text { including closure of donor site; }\end{array}$ & 19367 \\
\hline $\begin{array}{l}\text { Breast reconstruction with transverse rectus } \\
\text { abdominis myocutaneous flap (TRAM), single pedicle, } \\
\text { including closure of donor site; with microvascular } \\
\text { anastomosis (supercharging) }\end{array}$ & 19368 \\
\hline $\begin{array}{l}\text { Breast reconstruction with transverse rectus } \\
\text { abdominis myocutaneous flap (TRAM, double pedicle, } \\
\text { including closure of donor site) }\end{array}$ & 19369 \\
\hline
\end{tabular}

*CPT, Current Procedural Terminology 
Table, Supplementary Digital Content 2. International Classification of Diseases, Ninth Revision, Clinical Modification (ICD-9-CM) cause of unplanned reoperation and readmission codes.

\begin{tabular}{ll}
\hline Diagnostic descriptions & ICD-9-CM* codes \\
\hline Flap complication & $996.49,996.52,996.74,996.79$ \\
Infection & $682.2,996.69,998.5,998.59$ \\
Wound disruption & $879.0,879.1,998.30,998.31,998.32,998.83$ \\
Hemorrhage & 998.11 \\
Hematoma & $729.92,998.12$ \\
Seroma & $729.91,998.13,998.5$ \\
Postoperative pain & $338.18,338.28$ \\
\hline
\end{tabular}

*ICD-9-CM, International Classification of Diseases, Ninth Revision, Clinical Modification 


\section{REFERENCES}

1. Kamali P, Koolen PG, Ibrahim AM, et al. Analyzing Regional Differences over a 15-Year Trend of OneStage versus Two-Stage Breast Reconstruction in 941,191 Postmastectomy Patients. Plast Reconstr Surg 2016;138:1e-14e.

2. Ibrahim AM, Shuster M, Koolen PG, et al. Analysis of the National Surgical Quality Improvement Program database in 19,100 patients undergoing implant-based breast reconstruction: complication rates with acellular dermal matrix. Plast Reconstr Surg. 2013 Nov;132(5):1057-66.

3. Ogunleye AA, de Blacam C, Curtis MS, Colakoglu S, Tobias AM, Lee BT. An analysis of delayed breast reconstruction outcomes as recorded in the American College of Surgeons National Surgical Quality Improvement Program. J Plast Reconstr Aesthet Surg. 2012 Mar;65(3):289-94.

4. Pien I, Caccavale S, Cheung MC, et al. Evolving Trends in Autologous Breast Reconstruction: Is the Deep Inferior Epigastric Artery Perforator Flap Taking Over? Ann Plast Surg 2016;76:489-493.

5. Habermann EB, Thomsen KM, Hieken TJ, Boughey JC. Impact of availability of immediate breast reconstruction on bilateral mastectomy rates for breast cancer across the United States: data from the nationwide inpatient sample. Ann Surg Oncol 2014;21:3290-3296.

6. Wexelman B, Schwartz JA, Lee D, Estabrook A, Ma AM. Socioeconomic and geographic differences in immediate reconstruction after mastectomy in the United States. Breast J 2014;20:339-346.

7. Tuggle CT, Patel A, Broer N, Persing JA, Sosa JA, Au AF. Increased hospital volume is associated with improved outcomes following abdominal-based breast reconstruction. I Plast Surg Hand Surg 2014;48:382-388.

8. Masoomi H, Clark EG, Paydar KZ, et al. Predictive risk factors of free flap thrombosis in breast reconstruction surgery. Microsurgery 2014;34:589-594.

9. Albornoz CR, Bach PB, Mehrara BJ, et al. A paradigm shift in U.S. Breast reconstruction: increasing implant rates. Plast Reconstr Surg 2013;131:15-23.

10. Cemal Y, Albornoz CR, Disa JJ, et al. A paradigm shift in U.S. breast reconstruction: Part 2. The influence of changing mastectomy patterns on reconstructive rate and method. Plast Reconstr Surg 2013;131:320e-326e.

11. Frasier LL, Holden S, Holden T, et al. Temporal Trends in Postmastectomy Radiation Therapy and Breast Reconstruction Associated With Changes in National Comprehensive Cancer Network Guidelines. JAMA Oncol 2016;2:95-101.

12. Lang JE, Summers DE, Cui $H$, et al. Trends in post-mastectomy reconstruction: a SEER database analysis. Journal of surgical oncology 2013;108:163-168.

13. Agarwal S, Pappas L, Neumayer L, Agarwal J. An analysis of immediate postmastectomy breast reconstruction frequency using the surveillance, epidemiology, and end results database. Breast J 2011;17:352-358.

14. Fischer JP, Wes AM, Tuggle CT, et al. Mastectomy with or without immediate implant reconstruction has similar 30-day perioperative outcomes. J Plast Reconstr Aesthet Surg 2014;67:1515-1522.

15. Nwaogu I, Yan Y, Margenthaler JA, Myckatyn TM. Venous Thromboembolism after Breast Reconstruction in Patients Undergoing Breast Surgery: An American College of Surgeons NSQIP Analysis. J Am Coll Surg 2015;220:886-893.

16. Chung CU, Wink JD, Nelson JA, Fischer JP, Serletti JM, Kanchwala SK. Surgical Site Infections after Free Flap Breast Reconstruction: An Analysis of 2,899 Patients from the ACS-NSQIP Datasets. J Reconstr Microsurg 2015;31:434-441.

17. Silva AK, Lapin B, Yao KA, Song DH, Sisco M. The Effect of Contralateral Prophylactic Mastectomy on Perioperative Complications in Women Undergoing Immediate Breast Reconstruction: A NSQIP Analysis. Ann Surg Oncol 2015;22:3474-3480.

18. Kamali P, Curiel D, van Veldhuisen $C L$, et al. Trends in immediate breast reconstruction and early complication rates among older women: A big data analysis. J Surg Oncol. 2017 Jun;115(7):870-877. 
19. Kamali P, Zettervall SL, Wu W, et al. Differences in the Reporting of Racial and Socioeconomic Disparities among Three Large National Databases for Breast Reconstruction. Plast Reconstr Surg 2017;139:795807.

20. Kamali P, Paul MA, Ibrahim AM, et al. National and Regional Differences in 32,248 Postmastectomy Autologous Breast Reconstruction Using the Updated National Inpatient Survey. Ann Plast Surg 2017.

21. de Blacam C, Ogunleye AA, Momoh AO, et al. High body mass index and smoking predict morbidity in breast cancer surgery: a multivariate analysis of 26,988 patients from the national surgical quality improvement program database. Ann Surg. 2012 Mar;255(3):551-5.

22. Bleicher RJ, Ruth K, Sigurdson ER, et al. Time to Surgery and Breast Cancer Survival in the United States. JAMA Oncol 2016;2:330-339.

23. Fischer JP, Wes AM, Kovach SJ. The impact of surgical resident participation in breast reduction surgery-outcome analysis from the 2005-2011 ACS-NSQIP datasets. J Plast Surg Hand Surg 2014;48:315-321.

24. Nelson JA, Fischer JP, Chung CU, et al. Obesity and early complications following reduction mammaplasty: an analysis of 4545 patients from the 2005-2011 NSQIP datasets. J Plast Surg Hand Surg 2014;48:334-339.

25. Kim K, Mella JR, Ibrahim AMS, Koolen PGL, Lin SJ. Is There an Association between Component Separation and Venous Thromboembolism? Analysis of the NSQIP. Plastic and Reconstructive Surgery Global Open. 2015;3(6):e429. doi:10.1097/GOX.0000000000000167.

26. Kim K, Ibrahim AM, Koolen PG, Seyidova N, Lin SJ. Analysis of morbidity and mortality in patients undergoing skull base reconstruction. J Craniofac Surg. 2015 Jan;26(1):135-40.

27. Koolen PG, Ibrahim AM, Kim K, et al. Patient selection optimization following combined abdominal procedures: analysis of 4925 patients undergoing panniculectomy/abdominoplasty with or without concurrent hernia repair. Plast Reconstr Surg. 2014 Oct;134(4):539e-50e.

28. Kim K, Ibrahim AM, Koolen PG, Lee BT, Lin SJ. Trends in facial fracture treatment using the American College of Surgeons National Surgical Quality Improvement Program database. Plast Reconstr Surg. 2014 Mar;133(3):627-38.

29. Kim K, Ibrahim AM, Koolen PG, Frankenthaler RA, Lin SJ. Analysis of the NSQIP database in 676 patients undergoing laryngopharyngectomy: the impact of flap reconstruction. Otolaryngol Head Neck Surg. 2014 Jan;150(1):87-94.

30. Khuri SF. The NSQIP: A new frontier in surgery. Surgery. 2005;138:837-843.

31. American College of Surgeons. American College of Surgeons National Surgical Quality Improvement Program. Available at:http://site.acsnsqip.org/participants/. Accessed February 1, 2015.

32. Johnson C, Campwala I, Gupta S. Examining the validity of the ACS-NSQIP Risk Calculator in plastic surgery: lack of input specificity, outcome variability and imprecise risk calculations. J Investig Med. 2017 Mar;65(3):722-725.

33. O'Neill AC, Bagher S, Barandun M, Hofer SO, Zhong T. Can the American College of Surgeons NSQIP surgical risk calculator identify patients at risk of complications following microsurgical breast reconstruction? J Plast Reconstr Aesthet Surg. 2016 Oct;69(10):1356-62.

34. Luce EA, Pierce CE. Lack of Validity of the American College of Surgeons National Surgical Quality Improvement Program Database for Alloplastic Immediate Postmastectomy Reconstruction. Plast Reconstr Surg. 2015 Sep;136(3):296e-300e.

35. Sinha I, Pusic AL, Wilkins EG, et al. Late Surgical-Site Infection in Immediate Implant-Based Breast Reconstruction. Plast Reconstr Surg. 2017 Jan;139(1):20-28.

36. Cohen JB, Carroll C, Tenenbaum MM, Myckatyn TM. Breast Implant-Associated Infections: The Role of the National Surgical Quality Improvement Program and the Local Microbiome. Plast Reconstr Surg. 2015 Nov;136(5):921-9.

37. Duraes EF, Schwarz G, Durand P, et al. Complications Following Abdominal-Based Free Flap Breast Reconstruction: Is a 30 days Complication Rate Representative? Aesthetic Plast Surg. 2015 Oct;39(5):694-9. 
38. Albornoz CR, Cordeiro PG, Hishon L, et al. A nationwide analysis of the relationship between hospital volume and outcome for autologous breast reconstruction. Plast Reconstr Surg. 2013 Aug;132(2):192e200 .

39. Lin SJ, Nguyen MD, Chen C, et al. Tissue oximetry monitoring in microsurgical breast reconstruction decreases flap loss and improves rate of flap salvage. Plast Reconstr Surg. 2011 Mar;127(3):1080-5.

40. Ricci JA, Vargas CR, Lin SJ, Tobias AM, Taghinia AH, Lee BT. A Novel Free Flap Monitoring System Using Tissue Oximetry with Text Message Alerts. J Reconstr Microsurg. 2016 Jun;32(5):415-20.

41. Ricci JA, Vargas CR, Ho OA, Lin SJ, Tobias AM, Lee BT. Evaluating the Use of Tissue Oximetry to Decrease Intensive Unit Monitoring for Free Flap Breast Reconstruction. Ann Plast Surg. 2017 Jul;79(1):42-46.

42. Koolen PG, Vargas CR, Ho OA, et al. Does Increased Experience with Tissue Oximetry Monitoring in Microsurgical Breast Reconstruction Lead to Decreased Flap Loss? The Learning Effect. Plast Reconstr Surg. 2016 Apr;137(4):1093-101.

43. Unukovych D, Gallego CH, Aineskog H, Rodriguez-Lorenzo A, Mani M. Predictors of Reoperations in Deep Inferior Epigastric Perforator Flap Breast Reconstruction. Plast Reconstr Surg Glob Open. 2016 Aug 29;4(8):e1016.

44. Glass GE, Nanchahal J. Why haematomas cause flap failure: an evidence-based paradigm. J Plast Reconstr Aesthet Surg. 2012 Jul;65(7):903-10.

45. Halle M, Docherty Skogh AC, Friberg A, Edsander-Nord Å. Breast free flap complications related to haematoma formation - do the risks of multiple antithrombotics outweigh the benefits today? J Plast Surg Hand Surg. 2016 Aug;50(4):197-201.

46. Seth AK, Hirsch EM, Kim JY, et al. Hematoma after mastectomy with immediate reconstruction: an analysis of risk factors in 883 patients. Ann Plast Surg. 2013 Jul;71(1):20-3.

47. Mioton LM, Smetona JT, Hanwright PJ, et al. Comparing thirty-day outcomes in prosthetic and autologous breast reconstruction: a multivariate analysis of 13,082 patients? J Plast Reconstr Aesthet Surg. 2013 Jul;66(7):917-25. 


\section{Chapter}

\section{Prevalence of Psychiatric Comorbidities Among Women Undergoing Free Tissue Autologous Breast Reconstruction}

Babette E. Becherer

Parisa Kamali

Marek A. Paul

Winona Wu

Daniel A. Curiel

Hinne A. Rakhorst

Bernie Lee

Samuel J. Lin

Kari J. Kansal 


\section{ABSTRACT}

\section{Background}

Autologous breast reconstruction (BR) can be a stressful life event. Therefore, women undergoing mastectomy and autologous BR are required to have sufficient coping mechanisms. Although mental health problems are widespread, information regarding the prevalence of psychiatric diagnosis among these patients is scarce.

\section{Methods}

Retrospective analysis was performed using data from a large tertiary teaching hospital and the Nationwide Inpatient Sample (NIS) database. Patients undergoing autologous BR after mastectomy were included and evaluated for psychiatric disorders. Prevalence of each disorder, timing of diagnosis (preoperative or postoperative), and data per age group were reviewed.

\section{Results}

Between 2004 - 2014, 817 patients were included from the institutional database and 26,399 from the NIS database. Preoperatively, $15.3 \%$ of the patients were diagnosed with a psychiatric disorder within our institution and $17.6 \%$ nationwide $(p<.001)$. Postoperatively, $20.5 \%$ of the institutional patients were diagnosed with a psychiatric disorder. No major differences in prevalence were seen between age groups.

\section{Conclusion}

Approximately one in six patients were diagnosed with a psychiatric comorbidity preoperatively. Postoperatively, an additional 20.5\% developed a psychiatric disorder. There was no difference in prevalence and timing of diagnosis between age groups. 


\section{INTRODUCTION}

In 2015, approximately 231,840 new cases of invasive breast cancer and 60,290 new cases of breast carcinoma in situ (DCIS and LCIS) were diagnosed in the United States. ${ }^{1}$ Of women undergoing mastectomy, $21 \%$ to $42 \%$ opt for reconstructive breast surgery. ${ }^{2-}$ ${ }^{4}$ Although post-mastectomy breast reconstruction (BR) can have significant clinical and psychological benefits for patients, it remains a complex procedure with risk of severe complications, including loss of the reconstructed breast., ${ }^{5,6}$ Therefore, undergoing reconstructive breast surgery requires sufficient and long-lasting coping mechanisms to deal with the pre- and postoperative course, both physically and psychologically.

One of the most important factors influencing these coping mechanisms is the patients' preoperative mental health condition. ${ }^{7}$ Mental health problems are a widespread problem. In 2014, $18.1 \%$ of the U.S. population suffered from a mental disorder, varying per age group. ${ }^{8}$ This number increases in patients diagnosed with breast cancer, as previous studies describe that approximately 19\% - 42\% of these women suffer from mental health issues such as anxiety and depressive symptoms. ${ }^{9-13}$

Since the first described BR in 1895, methods have evolved into various different modalities from implant-based to autologous BR. ${ }^{14}$ In general, women undergoing autologous BR report higher long-term satisfaction rates and a better quality of life compared to women undergoing implant-based BR. ${ }^{15-19}$ Autologous BR should therefore be considered as a reconstructive option, if a patient qualifies, regardless of a psychiatric disorder. H However, due to the impact of the procedure, providers must be prepared for decompensation of any underlying mental problem.

To date, no detailed and conclusive information is available regarding the prevalence of psychiatric disorders among patients undergoing autologous BR. In this study, the authors aimed to (1) assess the prevalence of psychiatric comorbidities of patients undergoing autologous BR in a single institution and compare this to nationwide data, (2) discerned the timing of diagnosis of psychiatric disorders (before or after BR), and (3) identify any differences in prevalence between age groups.

\section{MATERIAL AND METHODS}

\section{Databases}

Patient data was obtained from an institutional database from the Division of Plastic and Reconstructive Surgery at Beth Israel Deaconess Medical Center (BIDMC), and the Healthcare and Utilization Project (HCUP) Nationwide Inpatient Sample (NIS) database. ${ }^{20}$ The institutional database included prospectively gathered data of all patients undergoing autologous BR, collected by plastic surgeons under institutional review board approval at BIDMC. The NIS database is the largest all-player inpatient 
care database in the United States, designed to represent $>95 \%$ of the U.S. population. It approximates a $20 \%$ stratified sample of all inpatient hospital discharges including all nonfederal, short-term, general, and both public and academic hospitals, based on hospital billing data. ${ }^{21}$

\section{Time frame}

The BIDMC institutional database provides data of all patients undergoing an autologous BR with at least one-year follow-up from 2004 - 2014. The NIS provides data from autologous BR cases from 2008 - 2012. For the evaluation of the prevalence of psychiatric comorbidities and the comparison between institutional and nationwide data, patients were selected from the overlapping years: 2008 - 2012. For the subgroup analysis of the timing of diagnosis of psychiatric disorders, and the subgroup analysis between age groups, the complete BIDMC database (2004 - 2014) was utilized. Since the NIS database registers inpatient stays only, it does not contain follow-up information, and therefore was not utilized for these subgroup analysis.

\section{Cohort selection}

Figure 1 demonstrates the patient selection process. Inclusion criteria entailed female patients $>18$ years of age, with breast cancer (International Classification of Diseases 2013, Ninth Revision (ICD-9) diagnosis codes 174.0 - 174.9, 233.0) or increased risk of breast cancer (ICD-9 diagnosis codes v10.3, v16.3, v84.01) undergoing free flap autologous BR (ICD-9 procedure codes 85.73 - 85.76). To ensure unified comparison between the two cohorts, complexity of surgical procedure, risk of flap loss and the possible related stress-levels for the patients, the following four types of autologous BR were included: deep inferior epigastric artery perforator (DIEP) flap, gluteal artery perforator (GAP) flap, superficial inferior epigastric artery perforator (SIEA) flap, and free transverse rectus abdominis myocutaneous (fTRAM) flap. Male patients, those under 18 years of age, and those with unknown gender or age were excluded. All ICD-9 diagnosis and procedure codes are listed in Supplemental File 1. To warrant a unified comparison between the two databases, only patients treated in large teaching hospitals were selected from the NIS database (bed size $500+$ ). ${ }^{22}$ 


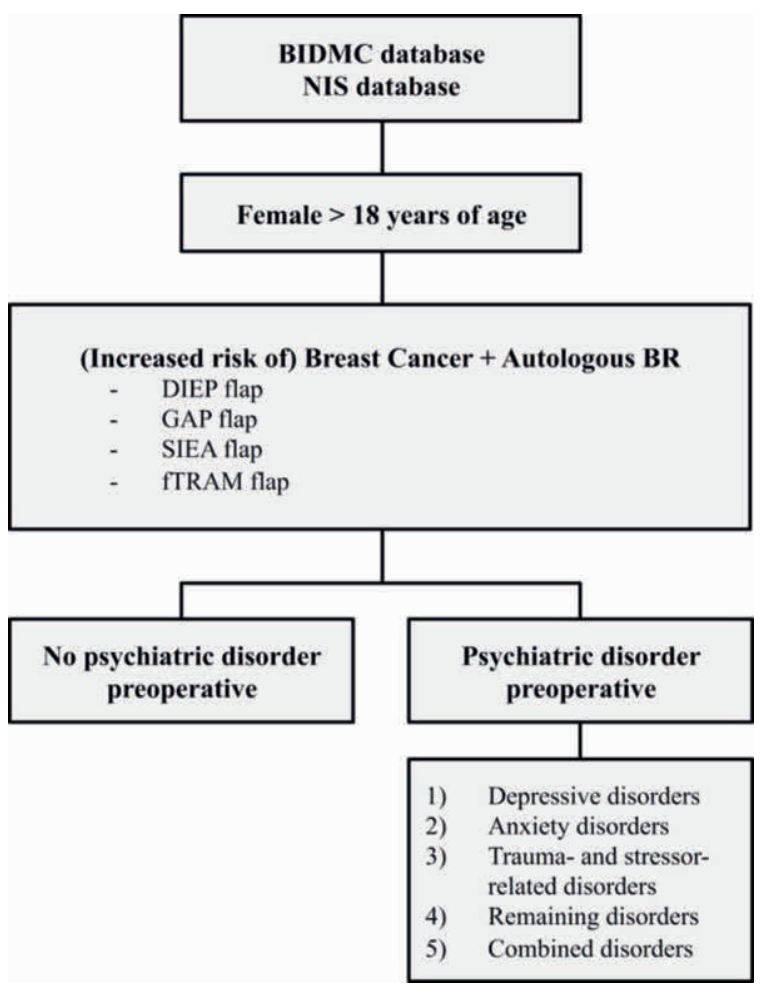

Figure 1 Inclusion criteria

\section{Analyzed variables}

The analyzed data included age at reconstruction, timing of BR (immediate or delayed), unilateral or bilateral BR, subtype of autologous reconstruction (DIEP flap, GAP flap, SIEA flap and fTRAM flap), tumor characteristics (DCIS, LCIS and Stage 1 - 3), prophylactic mastectomy, prevalence of a psychiatric disorder, and timing of diagnosis of psychiatric disorders in relation to the BR (Table 1$)$. 
Table 1. Patient demographics

\begin{tabular}{|c|c|c|c|c|c|}
\hline & $\begin{array}{l}\text { BIDMC } \\
(n=405)\end{array}$ & & $\begin{array}{l}\text { NIS } \\
(n=26,399)\end{array}$ & & $\mathrm{p}$ \\
\hline Age $(y)$ at $B R \pm S D$ & 50.3 & \pm 8.5 & 50.5 & \pm 8.9 & 0.243 \\
\hline$\leq 39$ & $9.9 \%$ & & $10.7 \%$ & & \\
\hline $40-49$ & $35.1 \%$ & & $35.8 \%$ & & \\
\hline $50-59$ & $41.2 \%$ & & $36.8 \%$ & & \\
\hline$\geq 60$ & $13.8 \%$ & & $16.7 \%$ & & \\
\hline Timing of BR & & & & & 0.325 \\
\hline Immediate BR & $60.0 \%$ & & $59.0 \%$ & & \\
\hline Delayed BR & $40.0 \%$ & & $41.0 \%$ & & \\
\hline Mastectomy & & & & & $<.001$ \\
\hline Unilateral & $57.0 \%$ & & $29.7 \%$ & & \\
\hline Bilateral & $43.0 \%$ & & $29.3 \%$ & & \\
\hline Unknown & $0 \%$ & & $41.0 \%$ & & \\
\hline Type of BR & & & & & $<.001$ \\
\hline DIEP & $95.1 \%$ & & $62.3 \%$ & & \\
\hline GAP & $4.0 \%$ & & $1.4 \%$ & & \\
\hline SIEA & $1.5 \%$ & & $3.3 \%$ & & \\
\hline fTRAM & $0.7 \%$ & & $32.9 \%$ & & \\
\hline \multicolumn{6}{|c|}{ Tumor characteristics } \\
\hline DCIS & $44.0 \%$ & & N/A & & \\
\hline LCIS & $5.6 \%$ & & N/A & & \\
\hline Stage 1 & $27.8 \%$ & & N/A & & \\
\hline Stage 2 & $21.9 \%$ & & N/A & & \\
\hline Stage 3 & $13.4 \%$ & & N/A & & \\
\hline Prophylactic & $36.7 \%$ & & N/A & & \\
\hline
\end{tabular}

BIDMC: Beth Israel Deaconess Medical Center database 2008 - 2012.

NIS: Nationwide Inpatient Sample database 2008 - 2012.

BR: breast reconstruction. N/A: description is not available in NIS database.

Age at reconstruction was in both databases divided into categories $\leq 39,40-49,50-59$ and $\geq 60$.

Timing of BR (immediate or delayed) was provided by the BIDMC database. Within the NIS database, patients undergoing autologous BR concurrent to mastectomy were defined as immediate BR and those only undergoing autologous BR as delayed BR. Unilateral or bilateral BR was provided in the BIDMC database. Within the NIS database, a bilateral or unilateral procedure was related to the concurrent bilateral or unilateral mastectomy based on ICD-9 codes. Without a simultaneous mastectomy, laterality could not be determined and was defined as unknown. Subtype of autologous breast reconstruction was provided in both databases. Tumor characteristics (DCIS, LCIS and Stage 1 - 3) and information about prophylactic mastectomy was provided in the BIDMC database. This data was not available in the NIS database. 


\section{Psychiatric disorders}

Prevalence of psychiatric disorders was obtained in both databases using ICD-9 diagnosis codes, correlating with the Diagnostic and Statistical Manual of Mental Disorders (DSM) criteria (Supplemental File 2). The registered psychiatric disorders in the NIS database are by default preoperative, as this dataset does not register follow-up information. To allow uniform comparison between both databases, only patients with a preoperative psychiatric disorder were seen as patients with a disorder within the BIDMC database.

Patients with a psychiatric disorder were divided into five subgroups: (1) depressive disorders, (2) anxiety disorders, (3) trauma- and stressor-related disorders, (4) remaining disorders (used for rarer diseases with a limited sample size such as substance-related or bipolar disorders), and the final category (5) combination of disorders (patients with several types of disorders).

To study the timing of diagnosis of psychiatric disorders in relation to the breast reconstructive procedure, the complete BIDMC database was used (2004 - 2014). By comparing the date of diagnosis with the date of BR the onset was categorized as either preoperative or postoperative. For the subgroup analysis of age groups, both preoperative and postoperative diagnosed disorders were selected from the complete BIDMC database (2004 - 2014), analyzed in relation to the number of women per age group.

\section{Statistical analysis}

In order to analyze multiple years of NIS data, the discharge weight factor provided by the NIS was utilized and national estimates were calculated by uniformly applying the weight factor to the discharges. ${ }^{21}$ Retrospective analysis was performed, and descriptive statistics were reported as proportions and compared using Pearson's Chi-square. All analysis were performed using SPSS 22.0 (IBM, Armonk, NY, USA) and statistical significance was set at $p<0.05$.

\section{RESULTS}

Between 2008 - 2012, 26,804 patients undergoing autologous breast reconstruction were identified and included, of which the BIDMC database comprised 405 patients and the NIS database 26,399 patients (Table 1). Mean age in years was $50.3 \pm 8.5$ in the BIDMC cohort and $50.5 \pm 8.9$ in the NIS cohort $(p=0.243)$. No significant differences were seen in timing of reconstruction (immediate or delayed) $(p=0.325)$. In the BIDMC cohort, $60.0 \%(n=243)$ of women underwent immediate reconstruction and $40.0 \%$ $(n=162)$ delayed reconstruction. Similar rates were seen in the NIS database: $59.0 \%$ $(n=15,575)$ immediate and $41.0 \%(n=10,824)$ delayed BR. Overall, within the BIDMC 
database, 57.0\% ( $n=231)$ underwent unilateral reconstruction, and 43.0\% $(n=174)$ bilateral reconstruction. Within the NIS cohort, only from patients undergoing immediate BR could be determined whether it was a unilateral $(29.7 \%, n=7,841)$ or bilateral reconstruction $(29.3 \%, n=7,734)$. In both databases, the most common autologous BR was a DIEP flap procedure: $95.1 \%(n=385)$ in the BIDMC database and $62.0 \%(n=16,367)$ in the NIS database.

Information about tumor characteristics was collected in the BIDMC database. Each diagnosis of cancer was addressed as separate incident; including patients who had bilateral breast cancer, multicentric cancer, or multifocal cancer. Overall, $49.6 \%$ of the patients underwent mastectomy and BR due to carcinoma in situ; $44.0 \%$ ( $n=178$ ) of the patients were diagnosed with DCIS and $5.6 \%(n=23)$ with LCIS. $63.1 \%$ of the patients had a diagnosis of invasive carcinoma at the time of surgery. Stage 1 was present in $27.8 \%$ $(n=113)$ of the women, stage 2 in $21.9 \%(n=89)$, and stage 3 in $13.4 \%(n=54)$. Of the women in this BIDMC cohort, $36.7 \%(n=149)$ underwent a unilateral or bilateral prophylactic mastectomy and reconstruction.

\section{Prevalence psychiatric disorders}

Between 2008 - 2012, 15.3\% ( $n=62)$ of the patients from the BIDMC cohort were diagnosed with one or more psychiatric disorders preoperatively. In the NIS database, the prevalence was $17.6 \%(n=4,646)$. No significant difference in prevalence was seen between both databases $(p=0.13$ ) (Table 2$)$. The distribution of type of psychiatric disorders differed between the two databases $(p<0.001)$, except for the group with a remaining disorder $(p=0.06)$. In the BIDMC cohort, a combination of disorders was most commonly seen $(8.1 \%, n=33)$. Of these, the majority $(50.8 \%, n=17)$ had a combination of a depressive disorder, trauma- and stressor-related disorder, and/or an anxiety disorder. The second most prevalent disorder was trauma- and stressor-related $(4.9 \%, n=20)$, followed by depressive disorders $(1.5 \%, n=6)$, anxiety disorders $(0.5 \%$, $\mathrm{n}=2$ ), and remaining disorders $(0.2 \%, \mathrm{n}=1)$ (Table 2$)$. Nationwide, depressive disorders were most prevalent $(8.4 \%, n=2,218)$, followed by anxiety disorders $(5.6 \%, n=1,478)$, a combination of disorders $(2.2 \%, n=581)$, remaining disorders (1.1\%, $n=290)$, and trauma- and stressor-related disorders $(0.3 \%, n=79)$ (Table 2$)$. 
Table 2. Prevalence of psychiatric disorders among women in the BIDMC database and NIS database, preoperative

\begin{tabular}{|c|c|c|c|c|c|}
\hline \multirow[b]{2}{*}{ No psychiatric diagnosis } & \multicolumn{2}{|c|}{$\begin{array}{l}\text { BIDMC \% (n) } \\
(n=405)\end{array}$} & \multicolumn{2}{|c|}{$\begin{array}{l}\text { NIS \% (n) } \\
(n=26,399)\end{array}$} & \multirow[t]{2}{*}{$\mathrm{p}$} \\
\hline & $84.7 \%$ & $(343)$ & $82.4 \%$ & $(21,753)$ & \\
\hline Total psychiatric diagnosis & $15.3 \%$ & $(62)$ & $17.6 \%$ & $(4,646)$ & 0.13 \\
\hline Depressive disorders & $1.5 \%$ & (6) & $8.4 \%$ & $(2,218)$ & $<.001$ \\
\hline Anxiety disorders & $0.5 \%$ & $(2)$ & $5.6 \%$ & $(1,478)$ & $<.001$ \\
\hline Trauma- and stressor-related disorders & $4.9 \%$ & $(20)$ & $0.3 \%$ & (79) & $<.001$ \\
\hline Remaining disorders & $0.2 \%$ & (1) & $1.1 \%$ & $(290)$ & 0.06 \\
\hline Combination of disorders & $8.1 \%$ & (33) & $2.2 \%$ & $(581)$ & $<.001$ \\
\hline
\end{tabular}

BIDMC: Beth Israel Deaconess Medical Center database 2008 - 2012.

NIS: Nationwide Inpatient Sample database 2008 - 2012.

Within the BIDMC cohort, preoperative prevalence of psychiatric disorders was not different between patients undergoing immediate or delayed reconstruction: $14.8 \%$ $(n=36)$ and $16.0 \%(n=26)$, respectively $(p=0.42)$. Prevalence of psychiatric disorders increased with higher tumor stage: $20.2 \%(n=18)$ in stage $1,21.7 \%(n=15)$ in stage 2 , and $27.9 \%(n=12)$ in stage $3(p=0.35)$.

In patients undergoing a breast cancer related procedure, prevalence of psychiatric comorbidities was higher $(17.3 \%, \mathrm{n}=44)$ than in patients opting for a prophylactic procedure $(12.2 \%, \mathrm{n}=18)$. However, this was not statistically significant $(p=0.11)$.

\section{Onset of psychiatric disorders}

To evaluate the timing of diagnosis for each type of psychiatric disorder, the complete BIDMC database was utilized (2004 - 2014), including 817 patients. Preoperatively, $14.7 \%(n=120)$ of the patients were diagnosed with a psychiatric comorbidity. Within the remaining group of 697 women without any psychiatric comorbidity preoperatively, an additional $20.5 \%(n=143)$ were diagnosed with one or more de novo psychiatric disorder after BR (Figure 2). Analysis within each type of psychiatric disorder demonstrates that time of onset differs per disorder (Figure 3). Overall, from all patients with a psychiatric disorder $(n=263)$, most of the trauma- and stressor-related disorders were seen preoperatively, whereas depressive and anxiety disorders were more frequently diagnosed postoperatively. 


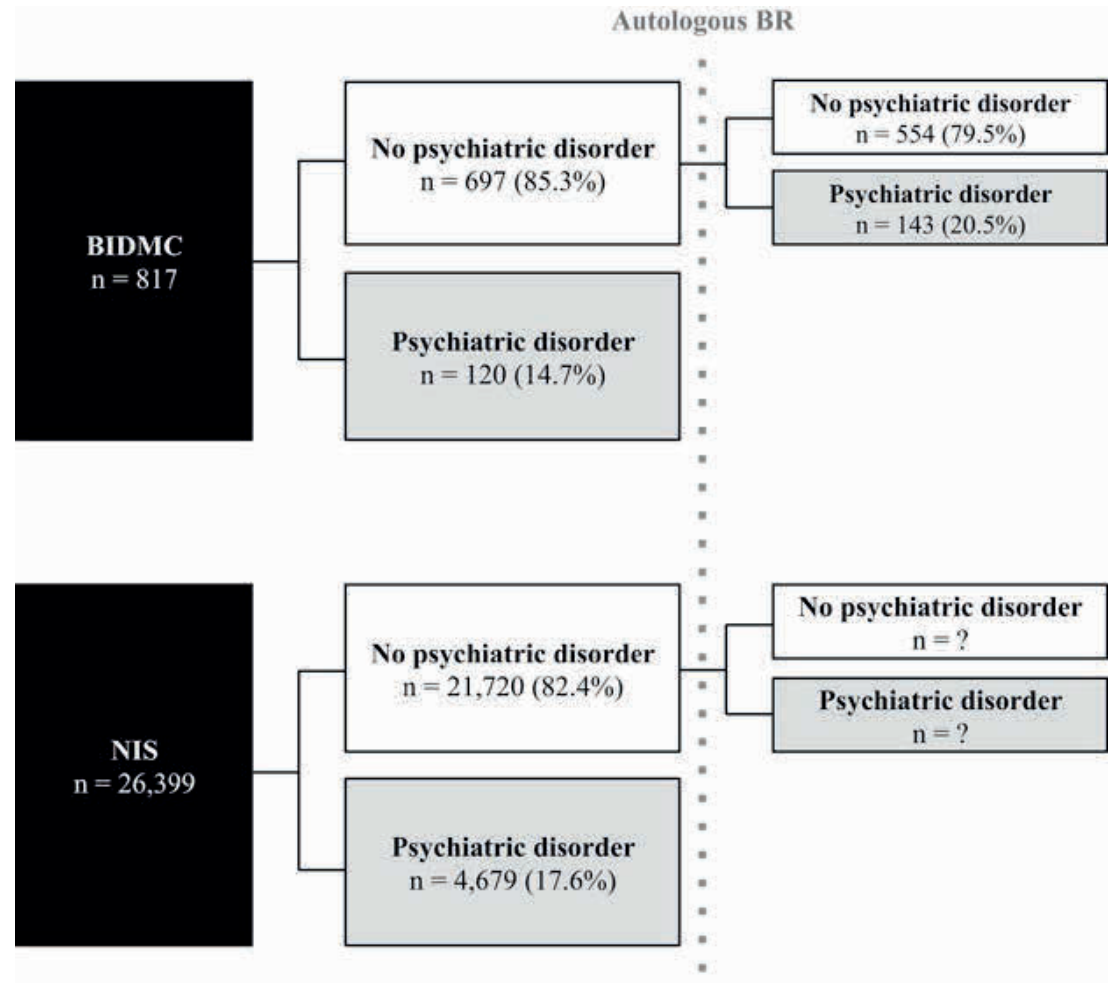

Figure 2. Pre- and postoperative prevalence of psychiatric disorders. BIDMC: Beth Israel Deaconess Medical Center database (2004 - 2014) NIS: Nationwide Inpatient Sample database (2008-2012)

\section{Diagnosis preoperative}

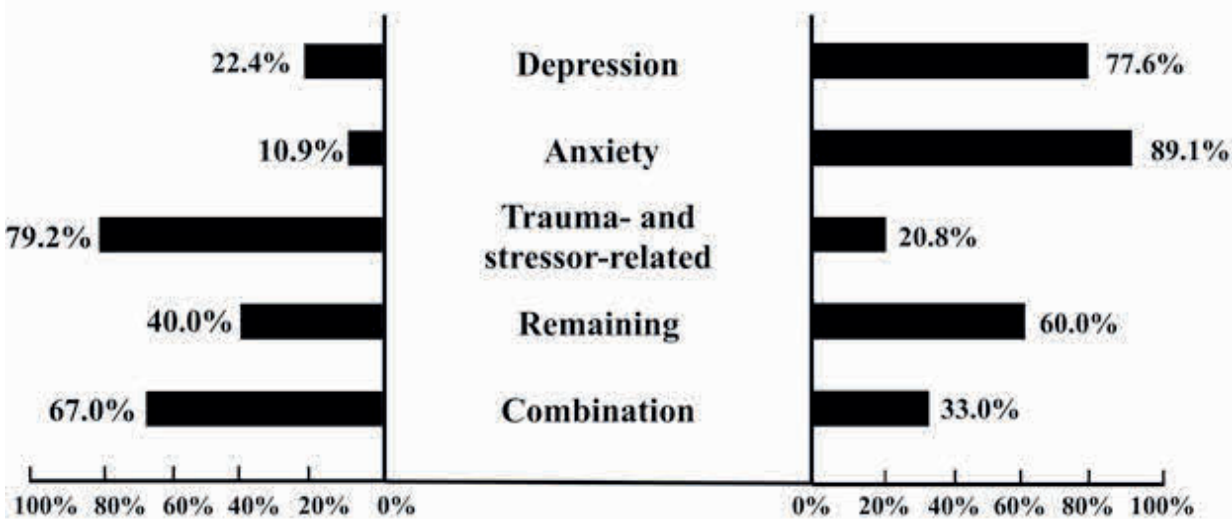

Figure 3. Moment of onset of psychiatric disorders, analyzed within each diagnosis group.

BIDMC database 2004 - $2014(n=263)$. Depression $(n=67)$, Anxiety ( $n=55)$, Trauma- and stressor-related $(n=48)$, remaining $(n=5)$, combination $(n=88)$. 


\section{Age group analysis}

Of all 817 women enrolled in the BIDMC database, 88 women were $\leq 39$ years of age, 316 between 40 - 49 years of age, 307 between 50 - 59, and 106 women were $\geq 60$ years of age.

Preoperatively, women who are over 60 years of age showed the highest total rate of psychiatric comorbidities $(16.0 \%, n=17)$, followed by women in the age groups $50-59$ $(14.7 \%, n=45)$ and $40-49(14.5 \%, n=46)$. Women $\leq 39$ years of age showed the lowest total prevalence $(13.6 \%, n=12)$ (Figure 4). Postoperatively, in all age groups, the prevalence of psychiatric comorbidities increased simultaneously with the increased time of follow-up. The highest increase was seen in women who are $\leq 39$ years of age: from $13.6 \%(n=12)$ to $36.4 \%(n=32)$. Concurrently, this subgroup is the group of women with the highest total rate of postoperative psychiatric comorbidities.

\section{Preoperative Postoperative}

\section{A. $<39$ years old $(n=88)$}

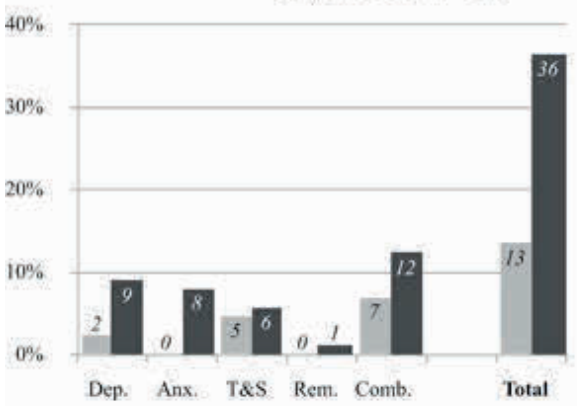

C. 50 - 59 years old $(n=307)$

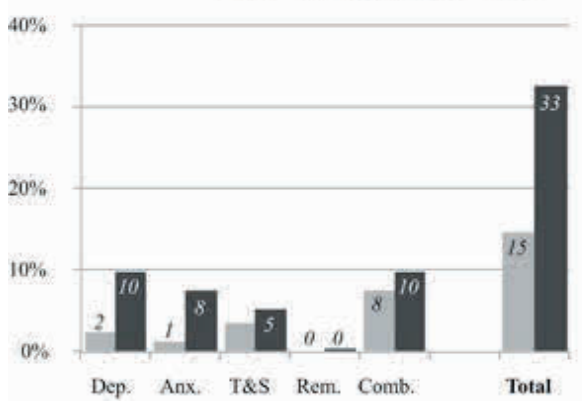

B. $40-49$ years old $(n=316)$

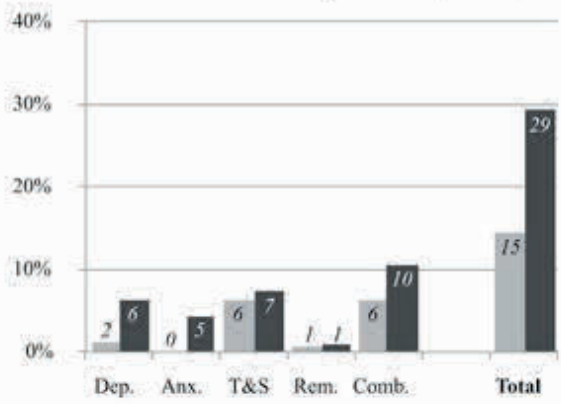

D. $>60$ years old $(n=106)$

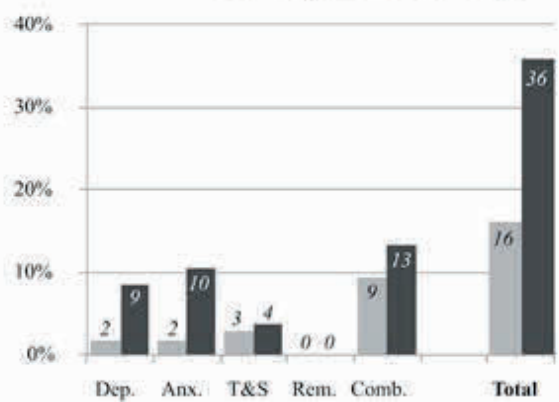

Figure 4. Pre- and postoperative prevalence of psychiatric disorders in percentage (\%), analyzed per age group.

BIDMC database 2004 - 2014 ( $\mathrm{n}=817$ ). Postoperative includes all patients with a psychiatric disorder preoperatively. Dep.: depressive disorder, Anx.: anxiety disorder, T\&S: trauma- and stressor-related disorder, Rem.: remaining disorder, Comb.: combined disorders. 


\section{DISCUSSION}

Cancer patients have a high probability of developing a stress-related psychiatric disorder. ${ }^{23-25}$ In addition, significant psychological and sexual implications are seen in breast cancer patients after a mastectomy. ${ }^{26}$ Metcalfe et al. demonstrated that in mastectomy patients stress might be reduced by the use of reconstructive breast surgery. ${ }^{6,27}$ While previous studies have focused on the psychological effect of BR and the quality of life, this study evaluated the general prevalence of psychiatric comorbidities in this patient group, both in the pre- and postoperative periods.

Our institutional data was shown to be representative for the nationwide female breast cancer population captured by the NIS database regarding the prevalence of known psychiatric disorders prior to BR. However, both databases report a lower prevalence of psychiatric disorders (15.3\% \& 17.6\%) than the average prevalence of mental health disorders in the general US female population, which is $21.8 \%$ as presented by the National Institution for Mental Health. ${ }^{8}$ Concurrently, this prevalence is lower than the number of women suffering from mental health issues while having a diagnosis of breast cancer or an increased risk of breast cancer, according to previous studies (19\% - 42\%). ${ }^{9-13}$ This difference may be due to inherent limitations in reviewing the data in retrospective fashion. Administrative databases are subject to variations in coding among providers, coding misclassification, and under-reporting. Possibly, the actual prevalence of psychiatric disorders - in both databases based on ICD-9 coding might be under-reported and thus underestimated. ${ }^{28,29}$

Sub-analysis within the BIDMC cohort showed no difference in preoperative prevalence of psychiatric disorders in patients opting for an immediate or delayed autologous BR. Further research is necessary to fully understand this information and should include analysis of a comparable patient group not undergoing breast reconstruction.

Postoperatively, the total prevalence of psychiatric comorbidities almost doubles (Figure 4), of which depressive and anxiety disorders are reported most frequently (Figure 3). Although exact reasons for the development of these mental health disorders were not analyzed in this study, various studies found that cancer-related concerns such as fear of recurrence, sexual functioning, and body image affect longterm psychosocial adjustment and play a major role in the quality of life after breast cancer treatment. ${ }^{30-33}$ On the other hand, a recent Dutch study showed no significant influence of BR on depressive symptoms, in women with a curative mastectomy for breast cancer. ${ }^{34}$ Therefore, it remains highly important to evaluate all potential provoking reasons for the development of a postoperative mental health problem in breast cancer patients who have undergone breast reconstructive surgery.

Interestingly, no major differences in the prevalence of psychiatric disorders were seen between the age groups. As presented by the National Institution of Mental Health, individuals aged 18 to 50 show higher numbers of mental health disorders than 
individuals $>50$ years of age. ${ }^{8}$ Our study reported comparable numbers of psychiatric disorders in all age groups preoperatively, ranging from 13.6\% - 16.0\% However, postoperatively, the highest increase in prevalence of psychiatric disorders was seen in women $\leq 39$ years of age. This younger age group may be more prone to mental health problems and could have more difficulties with adapting to a mastectomy with BR. ${ }^{31-33}$ Special attention to this particular group might be needed during follow-up.

As stated by Roth et al., psychological comorbidities have a negative influence on patients' postoperative BR impressions. ${ }^{35}$ However, patients who underwent BR report a better quality of life when compared to patients who underwent mastectomy alone. ${ }^{15-}$ 19 Although an autologous procedure might potentially lead to a higher number of secondary procedures in comparison to the expander-implant based options, women who underwent implant based reconstruction reported significantly decreased aesthetic satisfaction compared to autologous reconstruction over time. ${ }^{15,16}$ Considering this positive emotional aspect of reconstruction, every patient should have access to autologous BR, including women having mental health disorders.

In summary, a substantial proportion of breast cancer patients are diagnosed with psychiatric comorbidities. A psychiatric disorder can be pre-existent, or the stress of a breast cancer diagnosis and a BR itself might trigger any disorder. It is important to address a patients' underlying psychiatric disorder when preparing the patient for mastectomy and reconstructive surgery. Having appropriate counseling and psychological support before and after BR may help patients to stabilize their mental status throughout the complete breast cancer treatment. A multidisciplinary approach with a patient's psychiatrist, therapist or primary care physician is critical to help in these situations. Also, preparing the patient, her family, and providers for the possibility of an additional psychiatric disorder in the face of a breast cancer diagnosis may help in the overall well-being of the patient. Therefore, breast oncology and reconstructive surgeons should be aware of mental disturbances preoperatively, as well as postoperatively.

\section{Limitations}

The study is not exempt from limitations. First, the BIDMC database presents a relatively small patient group and was retrospectively analyzed. The NIS database is a large database covering a large patient group, but data collection is based on hospital billing data, resulting in possible under-reporting, over-reporting or misclassifications of variables. Furthermore, the NIS does not provide information about tumor characteristics or whether it is a prophylactic or tumor-related mastectomy; it only registers single inpatient hospital discharges, lacking follow-up information. At these points, our institutional database provided more insight. 


\section{CONCLUSION}

In conclusion, we found that approximately one in six patients (15.3\% institutional, $17.6 \%$ nationwide) were diagnosed with a psychiatric disorder before autologous BR. Postoperatively, one in every three patients was diagnosed with, or had overcome a psychiatric disorder. In general, trauma- and stressor-related disorders were mostly diagnosed before reconstruction, whereas depressive and anxiety disorders were more prevalent after BR. Women $\leq 39$ years of age showed the highest increase in prevalence of psychiatric disorders after BR.

Therefore, all patients undergoing autologous BR should be properly counseled and closely followed-up, with particular attention to mental health problems. Further analysis of the influence of any psychiatric comorbidity on choice of surgery, autologous $B R$ and evaluation of the patient related outcomes would be the logical next step. Future research has to be conducted to provide a more in-depth analysis of the influence of psychiatric disorders on the BR related complications and other variables like length of stay, additional surgeries, and patient satisfaction.

\section{FIGURE AND TABLE LEGEND}

Table 1. Patient demographics

Table 2. Prevalence of psychiatric disorders among women in the BIDMC database and NIS database, preoperative

Figure 1 Inclusion criteria

Figure 2. Pre- and postoperative prevalence of psychiatric disorders.

BIDMC: Beth Israel Deaconess Medical Center database (2004 - 2014)

NIS: Nationwide Inpatient Sample database (2008 - 2012)

Figure 3. Moment of onset of psychiatric disorders, analyzed within each diagnosis group.

BIDMC database $2004-2014(n=263)$. Depression ( $n=67)$, Anxiety $(n=55)$, Trauma- and stressor-related $(n=48)$, remaining $(n=5)$, combination $(n=88)$.

Figure 4. Pre- and postoperative prevalence of psychiatric disorders in percentage (\%), analyzed per age group. BIDMC database $2004-2014(n=817)$. Postoperative includes all patients with a psychiatric disorder preoperatively. Dep.: depressive disorder, Anx.: anxiety disorder, T\&S: trauma- and stressor-related disorder, Rem.: remaining disorder, Comb.: combined disorders.

Supplemental File 1. ICD-9 codes for breast cancer, increased risk of breast cancer and breast surgery procedures

Supplemental File 2. ICD-9 codes for psychiatric disorders 


\section{SUPPLEMENTAL FILES}

Supplemental File 1. ICD-9 codes for breast cancer, increased risk of breast cancer and breast surgery procedures

\begin{tabular}{|c|c|c|}
\hline Inclusion criteria & ICD-9 code & Description \\
\hline \multicolumn{3}{|l|}{ Breast cancer } \\
\hline \multirow[t]{3}{*}{ Increased risk } & v10.3 & Personal history of malignant neoplasm \\
\hline & v16.3 & Family history of malignant neoplasm \\
\hline & v84.01 & Genetic susceptibility to malignant neoplasm of breast \\
\hline \multirow[t]{2}{*}{ Breast cancer } & $174.0-.9$ & Malignant neoplasm of female breast \\
\hline & 233.0 & Carcinoma in situ of breast \\
\hline \multicolumn{3}{|l|}{ Mastectomy } \\
\hline & 85.40 & Mastectomy \\
\hline & v50.41 & Prophylactic removal of breast \\
\hline \multirow[t]{5}{*}{ Unilateral mastectomy } & 85.34 & Other unilateral subcutaneous mammectomy \\
\hline & 85.41 & Unilateral simple mastectomy \\
\hline & 85.43 & Unilateral extended simple mastectomy \\
\hline & 85.45 & Unilateral radical mastectomy \\
\hline & 85.47 & Unilateral extended radical mastectomy \\
\hline \multirow[t]{5}{*}{ Bilateral mastectomy } & 85.36 & Other bilateral subcutaneous mammectomy \\
\hline & 85.42 & Bilateral simple mastectomy \\
\hline & 85.44 & Bilateral extended simple mastectomy \\
\hline & 85.46 & Bilateral radical mastectomy \\
\hline & 85.48 & Bilateral extended radical mastectomy \\
\hline \multicolumn{3}{|c|}{ Autologous breast reconstruction } \\
\hline & 85.73 & Transverse rectus abdominis myocutaneous (TRAM) flap, free \\
\hline & 85.74 & Deep inferior epigastric artery perforator (DIEP) flap, free \\
\hline & 85.75 & Superficial inferior epigastric artery (SIEA) flap, free \\
\hline & 85.76 & Gluteal artery perforator (GAP) flap, free \\
\hline
\end{tabular}

ICD-9: International Classification of Diseases, 9th Revision, Clinical Modification codes. 


\section{Chapter 7}

Supplemental File 2. ICD-9 codes for psychiatric disorders

\begin{tabular}{lll}
\hline Psychiatric disorder & ICD-9 code & Description \\
\hline Depressive disorders & & \\
& 293.83 & Mood disorder in conditions classified elsewhere \\
& 296.1 & Manic disorder recurrent episode \\
$296.2-.3$ & Major depressive disorder single \& recurrent episode \\
296.81 & Atypical manic disorder \\
296.82 & Atypical depressive disorder \\
296.9 & Other and unspecified episodic mood disorder \\
300.4 & Dysthymic disorder \\
309.1 & Prolonged depressive reaction \\
311 & Depressive disorder, not elsewhere classified
\end{tabular}

Anxiety disorders

293.84 Anxiety disorder in conditions classified elsewhere

$300.0 \quad$ Anxiety states

$300.2 \quad$ Phobic disorders

$309.21 \quad$ Separation anxiety disorder

Trauma- and stressor-related disorders

309.0 Adjustment disorder with depressed mood

309.24 Adjustment disorder with anxiety

309.28 Adjustment disorder with mixed anxiety and depressed mood

$309.81 \quad$ Posttraumatic stress disorder

Remaining disorders

Substance-related and addictive disorders:

$291 \quad$ Alcohol-induced mental disorders

$292.1 \quad$ Drug-induced psychotic disorders

$292.2 \quad$ Pathological drug intoxication

$292.83 \quad$ Drug-induced persisting amnestic disorder

$292.84 \quad$ Drug-induced mood disorder

$292.89-.9$ Other specified \& unspecified drug-induced mental disorders

303 Alcohol dependence syndrome

$304 \quad$ Drug dependence

$305 \quad$ Nondependent abuse of drugs

Schizophrenia spectrum and other psychotic disorders:

$293.81-.82$ Psychotic disorder with delusions / hallucinations in conditions classified elsewhere

295 Schizophrenic disorders

297 Delusional disorders

$298 \quad$ Other nonorganic psychoses

Bipolar and related disorders:

$296.0 \quad$ Bipolar I disorder, single manic episode

296.4 - .7 Bipolar I disorder, most recent episode (or current) manic, depressed, mixed or unspecified

$296.80 \quad$ Bipolar disorder, unspecified 


\begin{tabular}{lll}
\hline Psychiatric disorder & ICD-9 code & Description \\
\hline Remaining disorders & & \\
& 296.89 & Other bipolar disorders \\
& Somatic symptom and related disorders: \\
300.1 & Dissociative, conversion and factitious disorders \\
300.5 & Neurasthenia \\
300.6 & Depersonalization disorder \\
300.7 & Hypochondriasis \\
300.81 & Somatization disorder \\
300.82 & Undifferentiated somatoform disorder \\
Neurocognitive disorders: & \\
301 & Personality disorders \\
\hline
\end{tabular}

ICD-9: International Classification of Diseases, 9th Revision, Clinical Modification codes. 


\section{REFERENCES}

1 American Cancer Society. Breast Cancer Facts \& Figures 2015-2016, www.cancer.org/acs/groups/content /@research/documents/document/acspc-046381.pdf; 2015 [accessed November 2015].

2 Greenberg CC, Lipsitz SR, Hughes ME, et al. Institutional variation in the surgical treatment of breast cancer: a study of the NCCN. Ann Surg 2011;254(2):339-45, DOI 10.1097/SLA.0b013e3182263bb0.

3 Morrow M, Li Y, Alderman AK, et al. Access to breast reconstruction after mastectomy and patient perspectives on reconstruction decision making. JAMA Surg 2014;149(10):1015-21, DOI 10.1001/jamasurg. 2014.548.

4 Reuben BC, Manwaring J, Neumayer LA. Recent trends and predictors in immediate breast reconstruction after mastectomy in the United States. Am J Surg 2009;198(2):237-43, DOI 10.1016/j.amjsurg. 2008.11.034.

5 Eltahir Y, Werners LL, Dreise MM, et al. Quality-of-life outcomes between mastectomy alone and breast reconstruction: comparison of patient-reported BREAST-Q and other health-related quality-of-life measures. Plast Reconstr Surg 2013;132(2):201e-9e, DOI 10.1097/PRS.0b013e31829586a7.

6 Metcalfe KA, Semple J, Quan ML, et al. Changes in psychosocial functioning 1 year after mastectomy alone, delayed breast reconstruction, or immediate breast reconstruction. Ann Surg Oncol 2012;19(1):233-41, DOI 10.1245/s10434-011-1828-7.

7 Roy-Byrne PP. Management of psychiatric disorders in patients with cancer, http://www.uptodate.com. proxy-ub.rug.nl/contents/management-of-psychiatric-disorders-in-patients-with-cancer?source=search_ result\&search=coping+and+cancer\&selectedTitle=3 150; 2016 [accessed April 2016].

8 Center for Behavioral Health Statistics and Quality, Behavioral health trends in the United States: Results from the 2014 National Survey on Drug Use and Health, http://www.samhsa.gov/data/; 2015 [accessed November 2015].

9 Burgess C, Cornelius V, Love S, et al. Depression and anxiety in women with early breast cancer: five year observational cohort study. BMJ 2005;330(7493):702, DOI 10.1136/bmj.38343.670868.D3.

10 Singer S, Schwentner L, van Ewijk R, et al. The course of psychiatric co-morbidity in patients with breast cancer - results from the prospective multi-centre BRENDA II study. Psychooncology 2015;25(5):590-6, DOI 10.1002/pon.3978.

11 Mitchell AJ, Chan M, Bhatti $\mathrm{H}$, et al. Prevalence of depression, anxiety, and adjustment disorder in oncological, haematological, and palliative-care settings: a meta-analysis of 94 interview-based studies. Lancet Oncol 2011;12(2):160-74, DOI 10.1016/S1470-2045(11)70002-X.

12 Mehnert A, Brahler E, Faller H, et al. Four-week prevalence of mental disorders in patients with cancer across major tumor entities. J Clin Oncol 2014;32(31):3540-6, DOI 10.1200/JCO.2014.56.0086.

13 Mclllmurray MB, Thomas C, Francis B, et al. The psychosocial needs of cancer patients: findings from an observational study. Eur J Cancer Care (Engl) 2001;10(4):261-9.

14 Schmauss D, Machens HG, Harder Y. Breast Reconstruction after Mastectomy. Front Surg 2016;2:71, DOI 10.3389/fsurg.2015.00071.

15 Atisha DM, Rushing CN, Samsa GP, et al. A national snapshot of satisfaction with breast cancer procedures. Ann Surg Oncol 2015;22(2):361-9, DOI 10.1245/s10434-014-4246-9.

16 Eltahir Y, Werners LL, Dreise MM, et al. Which breast is the best? Successful autologous or alloplastic breast reconstruction: patient-reported quality-of-life outcomes. Plast Reconstr Surg 2015;135(1):43-50, DOI 10.1097/PRS.0000000000000804.

17 Yueh JH, Slavin SA, Adesiyun T, et al. Patient satisfaction in postmastectomy breast reconstruction: a comparative evaluation of DIEP, TRAM, latissimus flap, and implant techniques. Plast Reconstr Surg 2010;125(6):1585-95, DOI 10.1097/PRS.0b013e3181cb6351.

18 Dean C, Chetty U, Forrest AP. Effects of immediate breast reconstruction on psychosocial morbidity after mastectomy. Lancet 1983;1(8322):459-62. 
19 Alderman AK, Kuhn LE, Lowery JC, Wilkins EG. Does patient satisfaction with breast reconstruction change over time? Two-year results of the Michigan Breast Reconstruction Outcomes Study. J Am Coll Surg 2007;204(1):7-12, DOI 10.1016/j.jamcollsurg.2006.09.022.

20 HCUP Databases. Healthcare Cost and Utilization Project (HCUP). Agency for Healthcare Research and Quality, Rockville, http://www.hcup-us.ahrq.gov/nisoverview.jsp; 2015 [accessed November 2015].

21 U.S. Department of Health \& Human Services, Agency for Healthcare Research and Quality (HCUP), NIS description of data elements, http://www.hcup-us.ahrq.gov/db/nation/nis/trendwghts.jsp; [accessed April 2016].

22 U.S. Department of Health \& Human Services, Agency for Healthcare Research and Quality (HCUP), NIS description of data elements, http://www.hcup-us.ahrq.gov/db/vars/hosp_bedsize/nisnote.jsp; [accessed April 2016].

23 Derogatis LR, Morrow GR, Fetting J, et al. The prevalence of psychiatric disorders among cancer patients. JAMA 1983;249(6):751-7.

24 Golden-Kreutz DM, Andersen BL. Depressive symptoms after breast cancer surgery: relationships with global, cancer-related, and life event stress. Psychooncology 2004;13(3):211-20, DOI 10.1002/pon.736.

25 Raison $\mathrm{CL}$, Miller AH. Depression in cancer: new developments regarding diagnosis and treatment. Biol Psychiatry 2003;54(3):283-94.

26 Jamison KR, Wellisch DK, Pasnau RO. Psychosocial aspects of mastectomy: I. the women's perspective. Am J Psychiatry 1978;135(4):432-6, DOI 10.1176/ajp.135.4.432.

27 Metcalfe KA, Esplen MJ, Goel V, Narod SA. Psychosocial functioning in women who have undergone bilateral prophylactic mastectomy. Psychooncology 2004;13(1):14-25, DOI 10.1002/pon.726.

28 Adkinson JM, Casale MT, Kim JY, et al. So You Have a Research Idea: A Survey of Databases Available for Plastic Surgery Research. Plast Reconstr Surg 2016;137(2):680-9, DOI 10.1097/01.prs.0000475794. 77102.ac.

29 Malay S, Shauver MJ, Chung KC. Applicability of large databases in outcomes research. J Hand Surg Am 2012;37(7):1437-46, DOI 10.1016/j.jhsa.2012.03.003.

30 Andersen BL, Shapiro CL, Farrar WB, et al. Psychological responses to cancer recurrence. Cancer 2005;104(7):1540-7, DOI 10.1002/cncr.21309.

31 Howard-Anderson J, Ganz PA, Bower JE, Stanton AL. Quality of life, fertility concerns, and behavioral health outcomes in younger breast cancer survivors: a systematic review. J Natl Cancer Inst 2012;104(5):386-405, DOI 10.1093/jnci/djr541.

32 Hartl K, Schennach R, Muller M, et al. Quality of life, anxiety, and oncological factors: a follow-up study of breast cancer patients. Psychosomatics 2010;51(2):112-23, DOI 10.1176/appi.psy.51.2.112.

33 Costanzo ES, Lutgendorf SK, Mattes ML, et al. Adjusting to life after treatment: distress and quality of life following treatment for breast cancer. Br J Cancer 2007;97(12):1625-31, DOI 10.1038/sj.bjc.6604091.

34 de Raaff CA, Derks EA, Torensma B, et al. Breast reconstruction after mastectomy: does it decrease depression at the long-term? Gland Surg 2016;5(4):377-84, DOI 10.21037/gs.2016.05.02.

35 Roth RS, Lowery JC, Davis J, Wilkins EG. Psychological factors predict patient satisfaction with postmastectomy breast reconstruction. Plast Reconstr Surg 2007;119(7):2008-15; discussion 2016-7, DOI 10.1097/01.prs.0000260584.09371.99. 



\section{Chapter}

\section{Differences in the Reporting of Racial and Socioeconomic Disparities among Three Large National Databases for Breast Reconstruction}

Parisa Kamali

Sara L. Zettervall

Winona W. Wu

Ahmed M. S. Ibrahim

Caroline Medin

Hinne A. Rakhorst

Marc L. Schermerhorn

Bernard T. Lee

Samuel J. Lin 


\section{ABSTRACT}

\section{Background}

Research derived from large-volume databases plays an increasing role in the development of clinical guidelines and health policy. In breast cancer research, the Surveillance, Epidemiology and End Result (SEER) program, National Surgical Quality Improvement Program (NSQIP) and the Nationwide Inpatient Sample (NIS) database are widely utilized. This study aims to compare the trends in immediate breast reconstruction (IBR) and identify the drawbacks and benefits of each database.

\section{Methods}

ICD-9 codes were used to identify patients with invasive breast cancer and ductal carcinoma In-Situ (DCIS) from 2005 to 2012 from each database. Trends of IBR over time were evaluated. Patient demographics and co-morbidities were compared. Subgroup analysis of IBR utilization per race was conducted.

\section{Results}

1.2 million patients were studied within the NIS, NSQIP and SEER. IBR in invasive breast cancer patients increased significantly over time from 2005 to 2012 in all databases (NIS $19.3 \%$ to $47.0 \%$, NSQIP $30.3 \%$ to $48.6 \%$, SEER $12.2 \%$ to $24.9 \%$, respectively; $P<0.001$ ). Similar significant upward trend was seen in DCIS patients. Significant disparities were reported in IBR rates among races White $(35.7 \%, 43.1 \%, 20.6 \% ; P<0.001)$, Black $(27.3 \%$, $30.7 \%$ and $15.5 \% ; P<0.001)$, Hispanic $(30.8 \%, 40.3 \%, 12.9 \% ; P<0.001)$ and Asian $(30.5 \%$, $32.1 \%, 14.0 \% ; P<0.001)$ in the NIS, NSQIP and SEER, respectively. Similar disparities were seen in the DCIS cohort. Rates of co-morbidities were similar in NIS, NSQIP and SEER.

\section{Conclusion}

There has been a significant increase in immediate breast reconstruction, however, the extent of the reporting of overall IBR rates and racial disparities differ significantly among databases. The NIS and NSQIP report similar findings with the SEER reporting results significantly lower in several categories. These findings suggest that utilization of SEER may not be universally generalizable to the entire US population. 


\section{INTRODUCTION}

In breast cancer research, the most utilized large national databases in breast cancer research are the Surveillance, Epidemiology and End Result (SEER) ${ }^{1}$ program, an epidemiologic surveillance system based on population-based tumor registries, the American College of Surgeon National Surgical Quality Improvement Program (NSQIP) ${ }^{2}$, a prospectively collected clinical database aiming to measure and improve the quality of surgical care across surgical specialties, and the Nationwide Inpatient Sample (NIS) database ${ }^{3}$, the largest administrative database within in the United States. All three databases have previously been used to study trends in surgical treatment of breast cancer and post-mastectomy breast reconstruction. ${ }^{4-39}$ These databases collectively offer unique opportunities to study patterns of care, variation in practice, and outcomes following surgical intervention. ${ }^{40,41}$ In recent years, research derived from these large databases have played increasing roles in the development of clinical guidelines and health policy. ${ }^{42}$ After the implementation of the Women's Health and Cancer Right Act in 1998, mandating health care payer coverage for post-mastectomy reconstructive surgery, a growing body of research utilized these large national databases and reported increased utilization of breast reconstruction but emphasized the geographic and racial disparities. 5,9,12,17,23,25,29,30,32,37-39,43,44 As a result, the "Breast Cancer Patient Education Act of 2015" was introduced in 2015 with the goal of increasing awareness on the availability and coverage of breast reconstruction, especially for racial and ethnic minority groups. ${ }^{45}$

With the increased use of these national databases for research, clinical guidelines and health policy, an understanding of the background, similarities, and fundamental differences between NIS, NSQIP, and SEER is necessary. Despite the robust data coming out of each database, few papers have evaluated the differences between these databases in regards to utilization of immediate breast reconstruction. Given this gap in knowledge, this study aims to (1) compare the trends in breast reconstruction from these databases and to evaluate whether similar trends are seen, (2) and to identify the drawbacks and benefit of each database.

\section{MATERIALS AND METHODS}

\section{Databases}

The Surveillance, Epidemiology and End Result (SEER) database is an epidemiologic surveillance system that was developed in $1973 .{ }^{1}$ SEER database is a population-based tumor registry designed to track cancer incidence, cancer directed surgery, radiation therapy provided for the first course of treatment and survival in 18 cancer registries across 15 states (Figure 1a). The Nationwide Inpatient Sample (NIS) database is an 
administrative database maintained by the Agency for Healthcare Research \& Quality with data available from 1988 to $2013{ }^{3}$ The NIS serves as the largest, administrative database within the United States, representing 20\% stratified sample of U.S. hospitals and included approximately 8 million annual admissions up to 2011, and all patients in the following years. Data in the NIS is collected from inpatient hospital stays from 47 states and is weighted to produce national estimates (Figure 1b). The National Surgical Quality Improvement Project (NSQIP) was created by the American College of Surgeons and has become a nationally validated registry. ${ }^{2}$ First developed in 2005, NSQIP provides a prospective, peer-controlled, database of patient demographics, comorbid conditions, operative variables, and 30-day postoperative surgical outcomes based clinical data for patients older than 15 years of age. In 2014, the database included data from 708 hospitals in 49 states (Figure 1c). The National Cancer Institutes Designated Cancer Centers and Comprehensive Cancer Centers are included in each map to reflect the distribution of large volume cancer centers with data included in each state represented (Figure $1 \mathrm{a}-\mathrm{c}){ }^{46}$

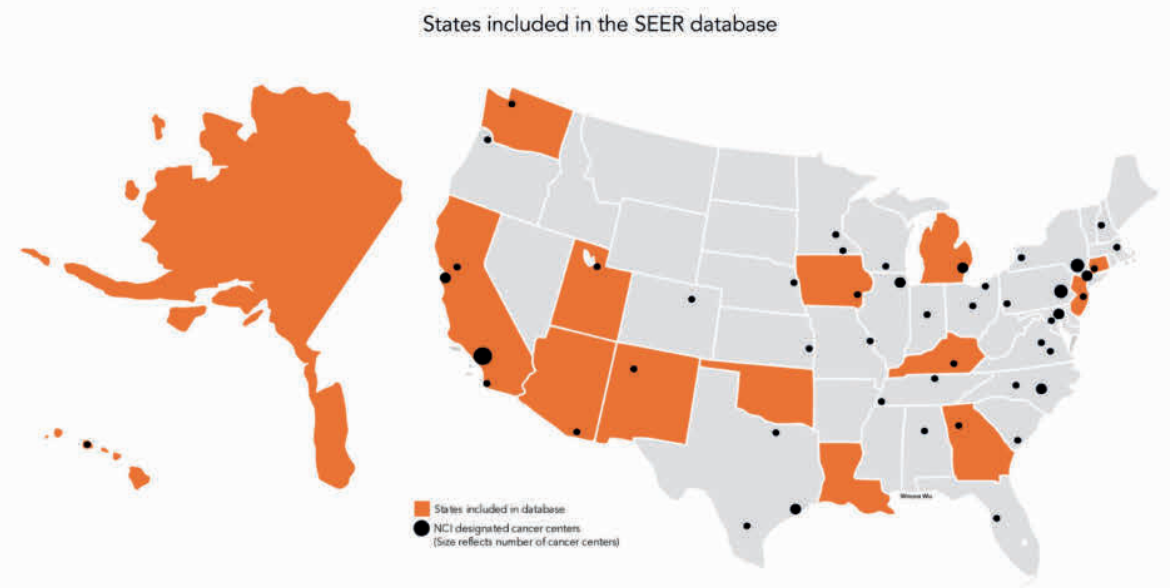

Figure 1a. Geographic map of the states included in the SEER database. 


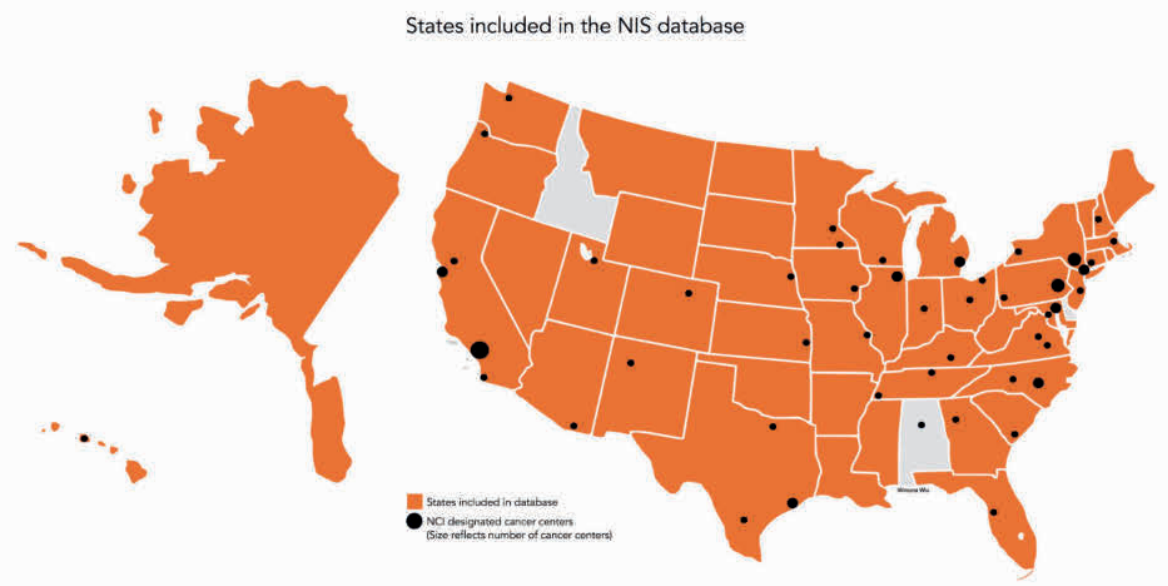

Figure 1b. Geographic map of the states included in the NIS database.

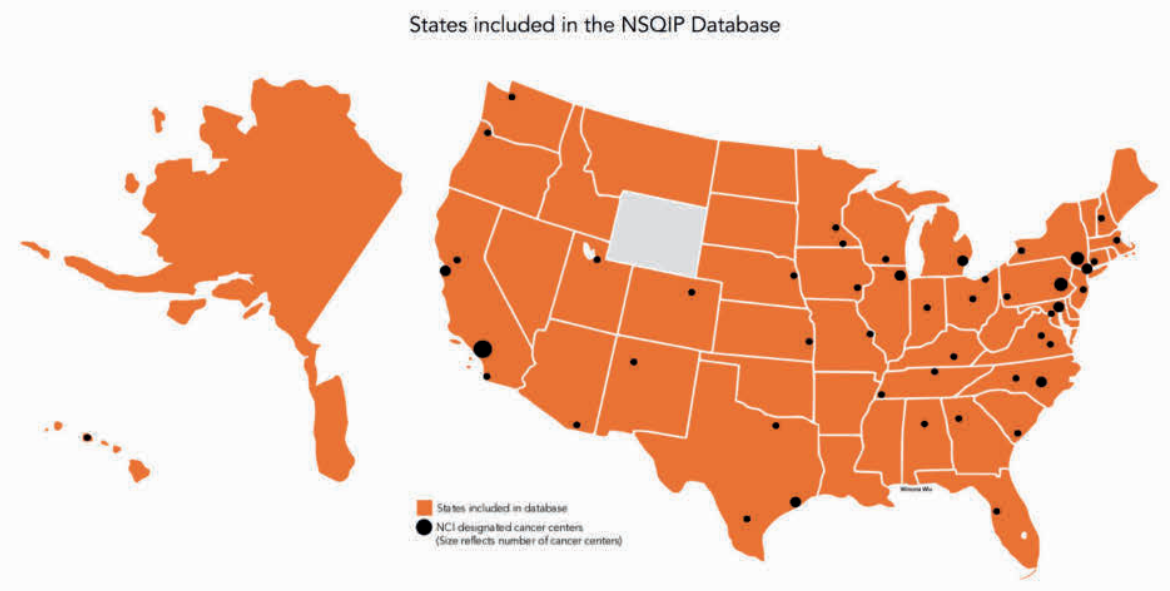

Figure 1c. Geographic map of the states included in the NSQIP database.

\section{PATIENT SELECTION}

All databases include International Classification of Diseases, Ninth Edition (ICD-9) diagnosis codes, which were utilized to identify patients with diagnoses of Ductal Carcinoma In situ (DCIS) (ICD-9 diagnosis code 233.0), or invasive breast cancer (ICD-9 diagnosis codes 174.0 to 174.9). The surgical procedure coding system available in each database (NIS: ICD-9 procedure coding, NSQIP: CPT coding, SEER: SEER Program Coding and Staging) was then utilized to identify patients undergoing subcutaneous, simple, 
radical, or extended radical mastectomy (Table, Supplemental Digital content 1 and 2). ${ }^{47-49}$ In both the NIS and the NSQIP, immediate breast reconstruction is defined as placement of tissue expander or permanent implant, autologous flap reconstruction or combined (autologous and implant-based) reconstruction concurrent with mastectomy (Table, Supplemental Digital content 3). In SEER, immediate breast reconstruction is defined as any type of breast reconstruction concurrent to, or within 4 months of mastectomy (Table, Supplemental Digital content 3). Patients under the age of 18 or without documented age, male patients, those undergoing outpatient procedures, and those without ICD-9 or procedure codes were excluded. Post-mastectomy immediate reconstruction rates and trend over time were evaluated separately for invasive breast cancer and DCIS patients.

To allow for uniform comparison among databases, demographic and comorbidity data was evaluated for the years 2005 - 2012. Moreover, in order illustrate immediate breast reconstruction utilization after the implementation of the Women's Health and Cancer Right Act of 1998, the trend over time is demonstrated from 1998 to 2012 for both SEER and NIS and from 2005 to 2014 for NSQIP. Subgroup analysis included stratification per race and a direct state-to-state comparison. Comparison between states was only possible in the SEER and NIS. We therefore compared rates of immediate breast reconstruction of the state of California for the SEER and the NIS. The state of California was chosen because of its highly diverse population. In SEER, the state of California included four cancer registries: Greater California (excluding San Francisco, Los Angeles and San Jose-Monterey), San Francisco-Oakland SMSA, San JoseMonterey, and the Los Angeles cancer registry. In the NIS, data from the state of California was provided by hospital ID.

\section{Variables}

Patient comorbidities were identified. Age and race were the only variable that was available in all three databases. Age was divided into categories $(<30,40-49,50-59,60-$ 69 and $>70)$. Race was classified into similar categories as census population data (White, Black, Hispanic, Asian or Pacific Islander and other). Diabetes mellitus, hypertension, chronic obstructive pulmonary disease, and obesity were available in both NIS and NSQIP.

\section{Statistical analysis}

Descriptive statistics were reported as proportions and compared using Pearson ChiSquare. Trends were analyzed using the Cochran-Armitage test for trend. All statistical analyses were performed using IBM SPSS version 22.0 (IBM Corp., Armonk, NY) and significance was set at $p<0.05$. 


\section{RESULTS}

\section{Invasive Breast Cancer}

Between 2005 and 2012, a total of 607,867 patients diagnosed with invasive breast cancer were identified in the NIS database. Among these patients who underwent mastectomy, 32.7\% $(n=151,660)$ opted for immediate reconstruction. When divided into type of reconstruction, 78.6\% ( $n=119,152)$ underwent implant-based, $13.7 \%$ $(n=20,764)$ autologous flap, and $7.7 \%(n=11,744)$ combined type breast reconstruction (Table 1). Immediate breast reconstruction increased significantly over time from $11.5 \%$ in 1998 to $47.0 \% 2012$ ( $p<0.001$ ) (Figure 2a).

The NSQIP database included 38,567 breast cancer patients of whom $40.7 \%$ $(n=13,372)$ underwent immediate breast reconstruction. Among all patients undergoing reconstruction, $77.2 \%(n=10,324)$ underwent implant-based breast reconstruction, $17.8 \%(n=2,376)$ autologous breast reconstruction and 5.0\% ( $n=672)$ combined breast reconstruction (Table 1 ). Immediate breast reconstruction also increased significantly over time in NSQIP; from 30.3\% in 2005 to $54.4 \%$ in 2014 ( $p<0.001$ ) (Figure 2a).

The SEER database included 439,564 breast cancer patients of whom $18.8 \%$ $(n=34,384)$ underwent immediate reconstruction. In total, $45.3 \%(n=15,587)$ underwent implant-based breast reconstruction, $41.2 \%(n=14,151)$ autologous reconstruction and $13.5 \%(n=4,646)$ combined type of reconstruction (Table 1). Immediate breast reconstruction increased significantly from $8.3 \%$ in 1998 to $24.9 \%$ in 2012 ( $p<0.001)$ (Figure 2a).

When demographic and comorbidities were evaluated, the average age differed between databases (NIS: $60.5 \pm 14.5$, NSQIP: $56.7 \pm 13.4$ SEER: $61.4 \pm 14.9$ ). Patients older than 70 years comprised $28.5 \%$ of patients in NIS, $21.3 \%$ in NSQIP, and $29.3 \%$ in SEER. In NSQIP, the most common age was 50-59 years (NIS: 23.6\% NSQIP: 26.2\%, SEER: $24.2 \%)$ When comparing comorbidities between the NIS and NSQIP, significant differences were found in hypertension $(41.0 \%$ and $39.8 \%$ respectively, $p<0.001$ ), diabetes mellitus (14.4\% and $10.8 \%$ respectively, $\mathrm{p}<0.001)$, and COPD $(5.0 \%$ and $2.6 \%$ respectively, $\mathrm{p}<0.001$ ) (Table 1 ).

Figure $3 a$ demonstrates the significant different rates of immediate breast reconstruction in breast cancer patients stratified per race within the NIS, NSQIP and SEER $(p<0.001)$. The highest rate of immediate breast reconstruction was seen among Caucasians; $35.7 \%$ in NIS, $43.1 \%$ in NSQIP, and $20.6 \%$ in SEER ( $p<0.001)$. The lowest rates of immediate breast reconstruction were found amongst Blacks: $27.3 \%$ in NIS, $30.7 \%$ in NSQIP, and $15.5 \%$ in SEER $(p<0.001)$. In the Hispanic population, the rates were $30.8 \%$ in NIS, $40.3 \%$ in NSQIP, and $12.9 \%$ in SEER $(p<0.001)$. Finally, within the Asian/Pacific Islander population, immediate breast reconstruction was utilized in $30.5 \%$ in NIS, 32.1\% in NSQIP, and $14.0 \%$ in SEER ( $p<0.001)$. 


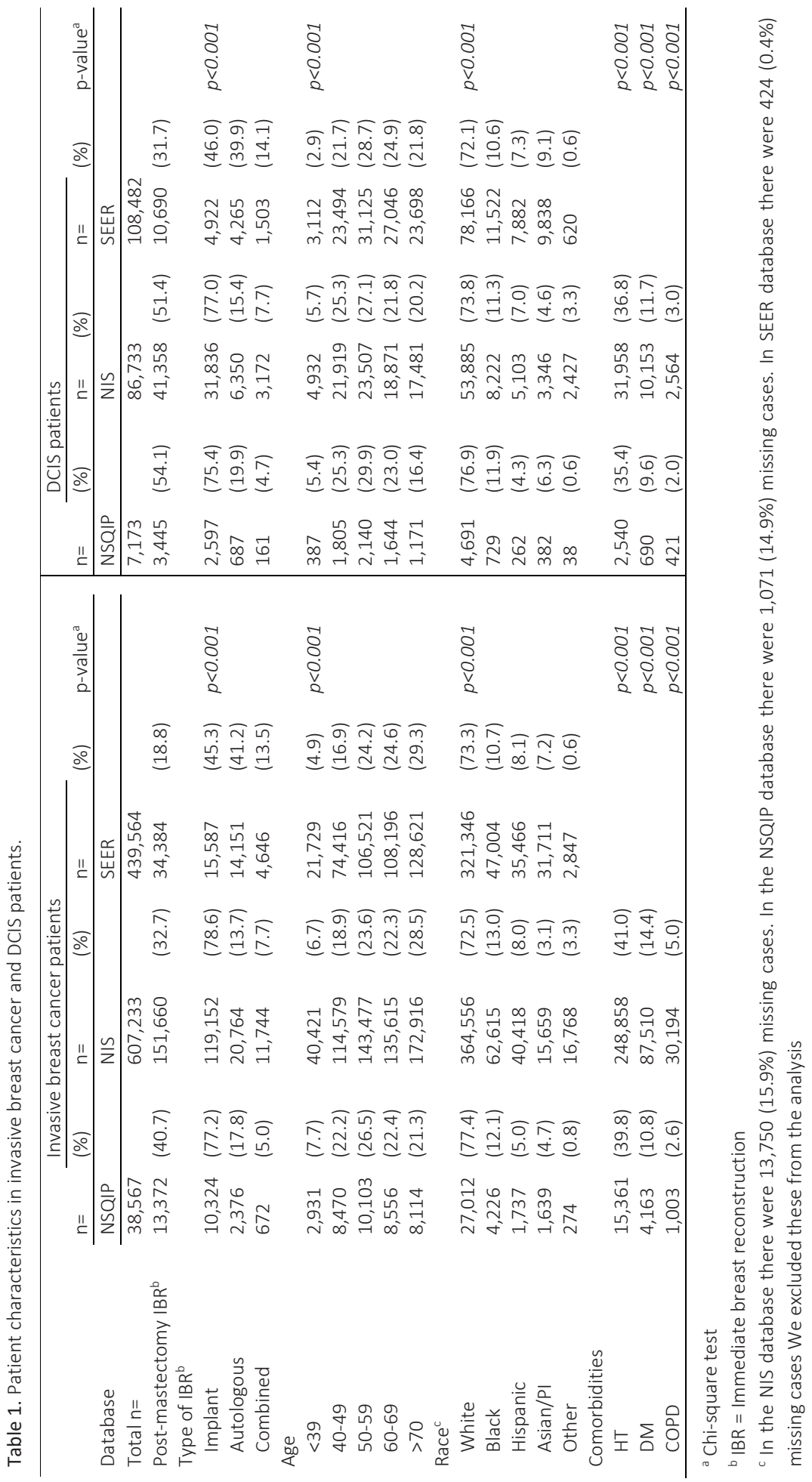


Immediate breast reconstruction in invasive breast cancer patients

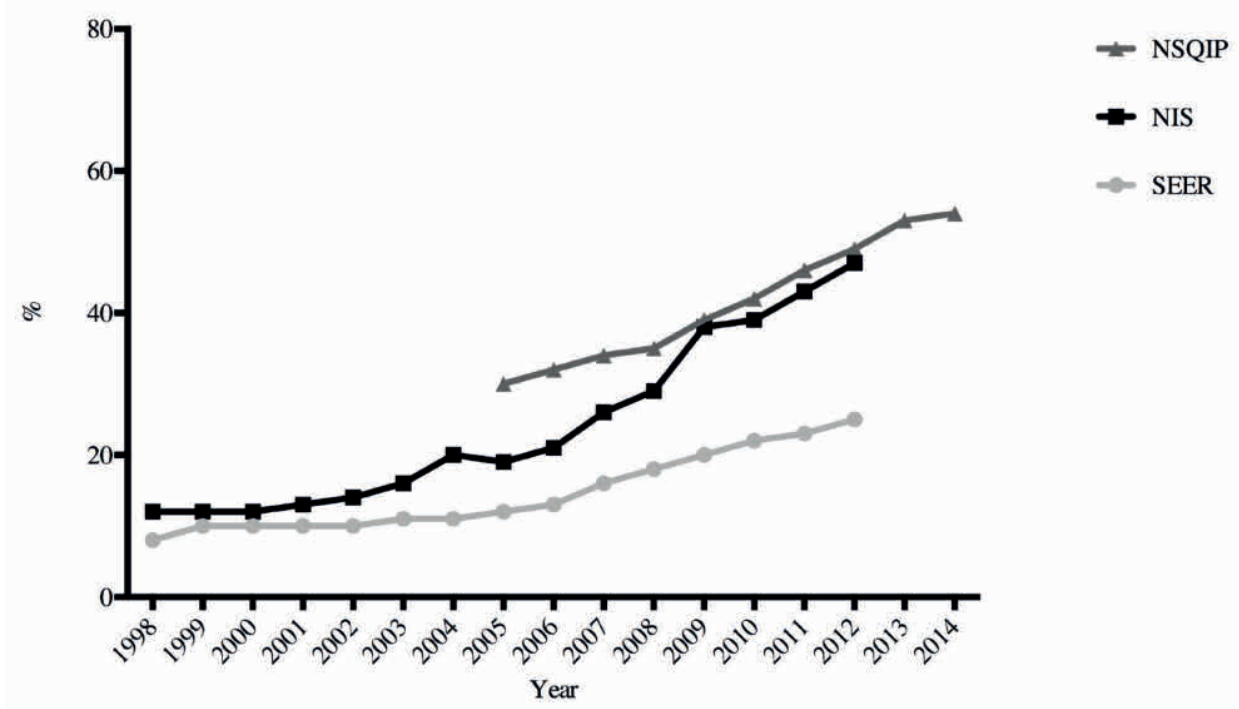

Figure 2a. Trend over time; Immediate breast reconstruction in invasive breast cancer patients.

*Cochrane-Armitage test for trend was $p<0.001$ in NIS, NSQIP and SEER.

Immediate breast reconstruction in DCIS patients

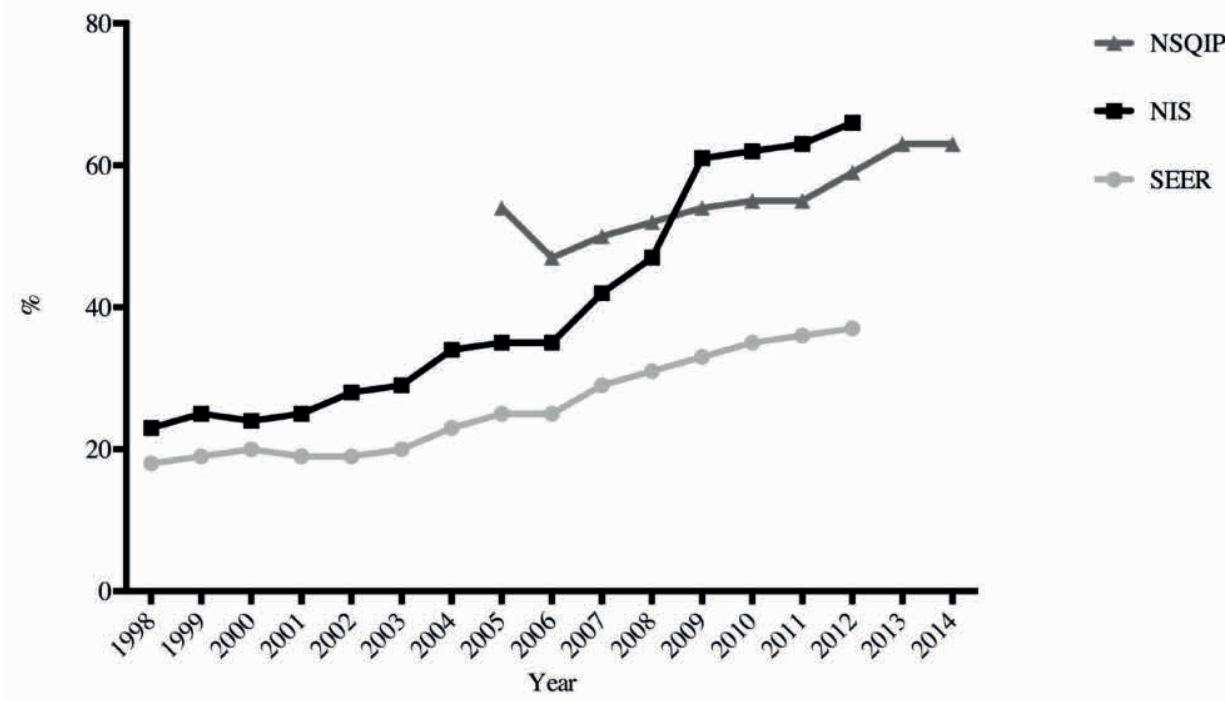

Figure $2 \mathrm{~b}$. Trend over time; Immediate breast reconstruction in DCIS patients.

*Cochrane-Armitage test for trend was $p<0.001$ in NIS, NSQIP and SEER. 
Immediate breast reconstruction in invasive breast cancer patients

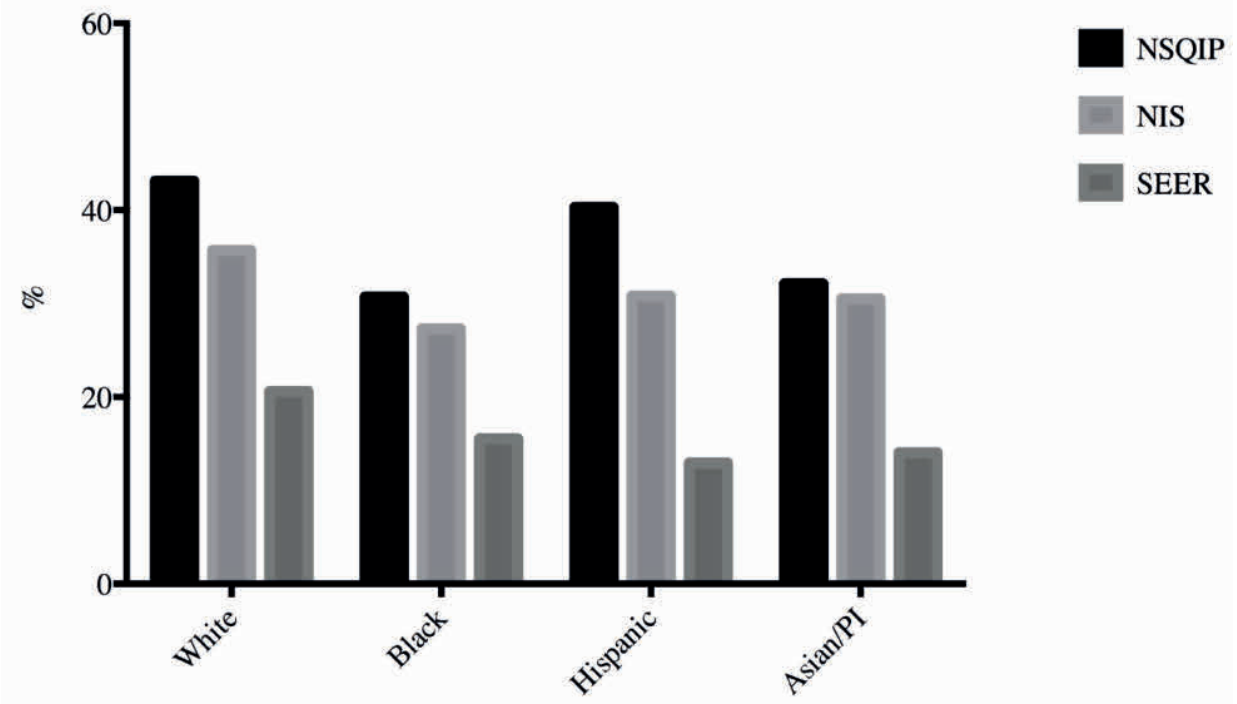

Figure 3a. Immediate breast reconstruction in invasive breast cancer patients stratified per race

Immediate breast reconstruction in DCIS patients
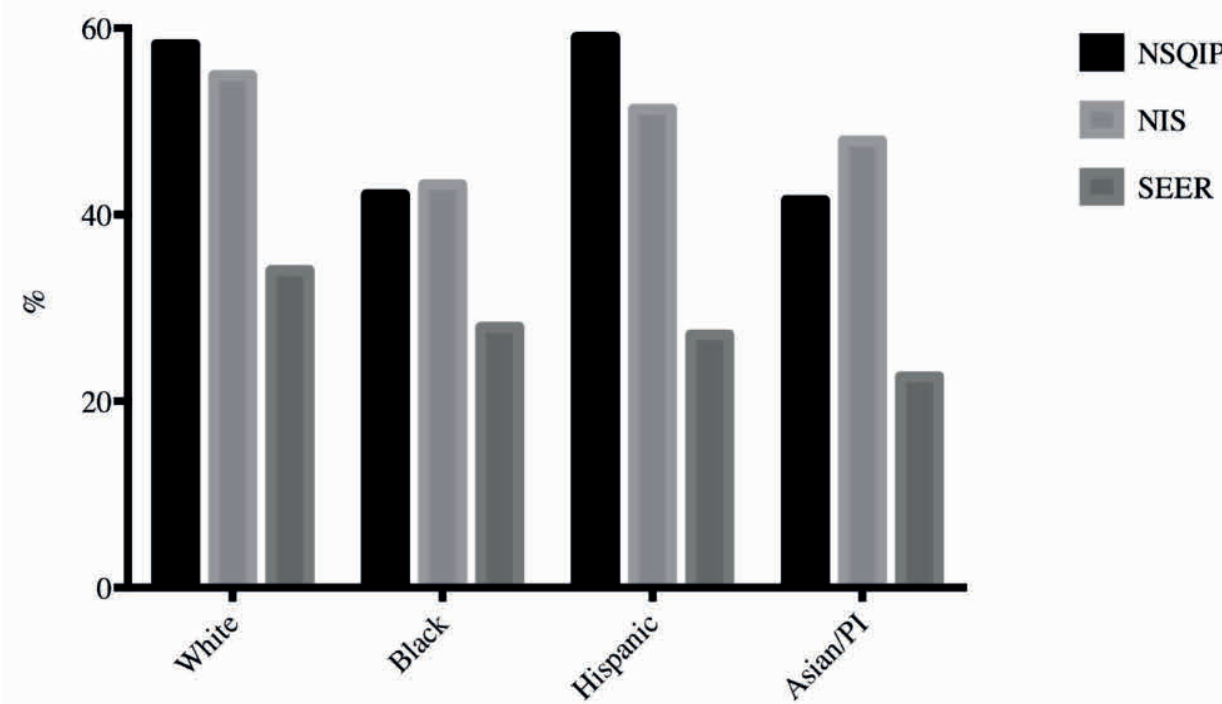

Figure $3 \mathrm{~b}$. Immediate breast reconstruction in DCIS patients stratified per race 


\section{DCIS patients}

NIS included 86,733 patients diagnosed with DCIS, of which $51.4 \%$ opted for immediate breast reconstruction. Implant-based breast reconstruction accounted for $77.0 \%$ $(n=31,836)$, autologous breast reconstruction for $15.4 \%(n=6,350)$ and combined type of breast reconstruction for $7.7 \%(n=3,172)$ (Table 1). Over time, immediate breast reconstruction increased significantly from $23.2 \%$ to $66.4 \%$ between 1998 to 2012, respectively $(p<0.001)$ (Figure $2 b)$.

NSQIP included 7,173 patients diagnosed with DCIS. 54.1\% $(n=3,445)$ of the patients treated with mastectomy underwent immediate reconstruction. Among all patients undergoing reconstruction, $75.4 \% \quad(n=2,597)$ underwent implant-based breast reconstruction, $19.9 \%(n=687)$ autologous breast reconstruction and $4.7 \%(n=116)$ combined breast reconstruction (Table 2). IBR rates increased significantly from 53.6 in 2005 to $62.6 \%$ in 2014 ( $p<0.001$ ) (Figure $2 b$ ).

Table 2. Overview strengths and weaknesses NIS, NSQIP and SEER database.

\begin{tabular}{|c|c|c|c|}
\hline & NIS & NSQIP & SEER \\
\hline Type of database & $\begin{array}{l}\text { Administrative database, } \\
\text { retrospective data }\end{array}$ & $\begin{array}{l}\text { Clinical database, } \\
\text { prospectively collected }\end{array}$ & $\begin{array}{l}\text { Epidemiologic data from } \\
\text { population-based cancer } \\
\text { registries. }\end{array}$ \\
\hline Data collected & Hospital discharge claims & \multicolumn{2}{|c|}{$\begin{array}{ll}\text { Directed review of medicalCancer registry data from } \\
\text { records of randomly } & \text { National Cancer Institute's } \\
\begin{array}{l}\text { assigned patients in } \\
\text { participating hospitals }\end{array} & \text { sponsored institutions }\end{array}$} \\
\hline Diagnosis identification & \multicolumn{3}{|c|}{ 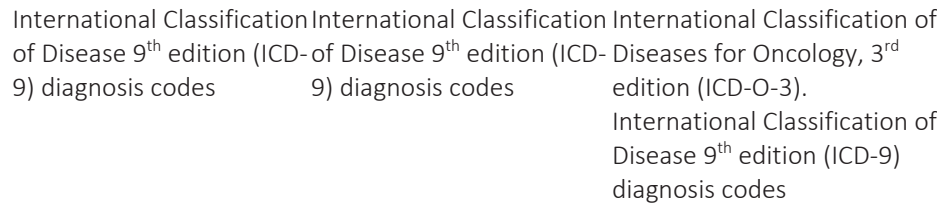 } \\
\hline Procedure identification & \multicolumn{2}{|c|}{$\begin{array}{l}\text { International Classification CPT codes } \\
\text { of Disease } 9^{\text {th }} \text { edition (ICD- } \\
\text { 9) diagnosis codes }\end{array}$} & $\begin{array}{l}\text { SEER Coding and Staging } \\
\text { Manual }\end{array}$ \\
\hline Variables & $\begin{array}{l}\text { Primary/secondary } \\
\text { diagnoses } \\
\text { Primary/secondary } \\
\text { procedures Admission/ } \\
\text { discharge status Patient } \\
\text { demographics } \\
\text { Provider/hospital } \\
\text { characteristics Cost, LOS, } \\
\text { insurance Inpatient } \\
\text { mortality }\end{array}$ & $\begin{array}{l}\text { Preoperative risk factors, } \\
\text { Intraoperative data, } \\
\text { Patient demographics, } \\
\text { Outcomes, Procedures, } \\
\text { 30-Day } \\
\text { morbidity/mortality }\end{array}$ & $\begin{array}{l}\text { Stage/date of cancer } \\
\text { diagnosis, Primary disease } \\
\text { site, tumor size, therapy, } \\
\text { Mortality, Demographics }\end{array}$ \\
\hline Geography & $\begin{array}{l}\text { Nationwide } \\
\text { (Figure 1a) }\end{array}$ & $\begin{array}{l}\text { Participating hospitals } \\
\text { (Figure } 1 \mathrm{~b} \text { ) }\end{array}$ & $\begin{array}{l}17 \text { cancer registries } \\
\text { (Figure } 1 \mathrm{c} \text { ) }\end{array}$ \\
\hline
\end{tabular}




\begin{tabular}{|c|c|c|c|}
\hline & NIS & NSQIP & SEER \\
\hline $\begin{array}{l}\text { Time range data } \\
\text { collection }\end{array}$ & Inpatient stays & $\begin{array}{l}\text { Inpatient and outpatient } \\
\text { up to } 30 \text { days post- } \\
\text { operative }\end{array}$ & $\begin{array}{l}\text { Longitudinal from diagnosis of } \\
\text { cancer until death }\end{array}$ \\
\hline Limitations & $\begin{array}{l}\text { Includes only inpatient } \\
\text { events, inpatient } \\
\text { morbidity and mortality } \\
\text { may be studied, }\end{array}$ & $\begin{array}{l}\text { Patient data are limited to } \\
\text { 30-days post discharge; } \\
\text { readmissions, } \\
\text { complications, and deaths } \\
\text { after this period are not } \\
\text { available. } \\
\text { Not all patients are } \\
\text { included, but rather a } \\
\text { select sampling of } \\
\text { patients. } \\
\text { Patients under the age of } \\
\text { 18, transplant cases, and } \\
\text { concurrent cases are } \\
\text { excluded. }\end{array}$ & $\begin{array}{l}\text { Lacks recurrence, radiation, } \\
\text { and chemotherapy data. } \\
\text { Data only collected from } \\
\text { institutional source. }\end{array}$ \\
\hline
\end{tabular}

The lowest rate of immediate breast reconstruction was seen in the SEER database. Of the 108,482 patients diagnosed with DCIS who underwent mastectomy, $31.7 \%$ $(n=10,690)$ opted for immediate breast reconstruction. A total of $46.0 \%(n=4,922)$ underwent implant-based breast reconstruction, 39.9\% ( $n=4,265)$ autologous reconstruction, and $14.1 \%(n=1,503)$ combined type of reconstruction. Over time, immediate reconstruction increased significantly from $18.1 \%$ in 1998 to $36.6 \%$ in 2012 $(p<0.001)$ (Figure $2 b)$.

The mean age was similar among databases (NIS: $57.7 \pm 13.0$, NSQIP: $56.7 \pm 12.3$, SEER: $59.2 \pm 13.5$ ), and the majority of women were aged $50-59$ years (NIS 26.3\%, NSQIP 29.4\%, SEER 28.5\%). Rates of comorbidities within the NIS and NSQIP were significantly different for hypertension $(36.8 \% \vee 35.4 \%, p<0.001)$, diabetes $(11.7 \% \vee$ $9.6 \%, p<0.001)$, and COPD (3.0\% v 2.0\%, p<0.001) (Table 2).

There were significant differences in utilization of immediate breast reconstruction utilization by DCIS patients stratified per race $(p<0.001)$. Caucasian patients opted for immediate breast reconstruction in $54.9 \%$ in the NIS, 58.2\% in the NSQIP, and $34.0 \%$ in the SEER population $(p<0.001)$. Black patients underwent immediate breast reconstruction less frequently (NIS: 43.2\%, NSQIP: 42.1\%, NIS: 43.2\%, SEER: $27.9 \%$ ) $(p<0.001)$. Hispanic patients underwent immediate breast reconstruction at rates of $51.3 \%$ in NIS, 59.0\% in NSQIP, and $27.1 \%$ in SEER ( $p<0.001)$. Finally, immediate breast reconstruction utilization in the Asian/Pacific Islander population was as follows; NIS: 47.9\% NSQIP: 41.5\%, and SEER: $22.6 \%$ ( $p<0.001)$.

\section{Comparison by state: California}

Within the SEER cohort, a total of 196,100 patients diagnosed with both invasive breast cancer or DCIS were treated in California, reflecting 35.8\% of the total SEER cohort. Of 
these, $81.4 \%$ ( $n=159,673)$ were diagnosed with invasive breast cancer and $18.6 \%$ $(n=36,427)$ with DCIS. Of the post-mastectomy cohort, $15.9 \%(n=10,493)$ of invasive breast cancer patients and $28.2 \%(n=3,176)$ DCIS patients underwent immediate breast reconstruction. In the NIS, the state of California comprised 78,714 patients, reflecting $11.3 \%$ of the complete NIS cohort. Of these, $86.4 \%(n=67,971)$ included patients diagnosed with breast cancer and $13.6 \%(n=10,743)$ patients diagnosed with DCIS. Of patients undergoing mastectomy, $31.6 \%(n=15,999)$ of invasive breast cancer patients and $52.5 \%(n=5,044)$ DCIS patients underwent immediate breast reconstruction.

Figure $4 a-b$ demonstrates the rates in immediate breast reconstruction utilization stratified per race. For invasive breast cancer patients, immediate breast reconstruction utilization differed significantly among all races: White (NIS; 35.0\%, SEER; 18.2\%) $(p<0.001)$, Black (NIS; 25.8\%, SEER; 11.9\%) ( $p<0.001$ ), Hispanic (NIS; 25.1\%, SEER; $10.8 \%)(p<0.001)$ and Asian/Pacific Islander population (NIS; 28.6\%, SEER; $13.2 \%$ ) $(p<0.001)$. Significant differences within all races was also seen in the DCIS population; White (NIS; 57.1\%, SEER; 23.5\%) ( $p<0.001$ ), Black (NIS; 46.0\%, SEER; 24.4\%) $(p<0.001)$, Hispanic (NIS; 38.9\%, SEER; 24.4\%) ( $p<0.001)$ and Asian/Pacific Islander (NIS; 50.3\%, SEER; $21.3 \%)(p<0.001)$.

\section{California: Immediate breast reconstruction in invasive breast cancer patients}

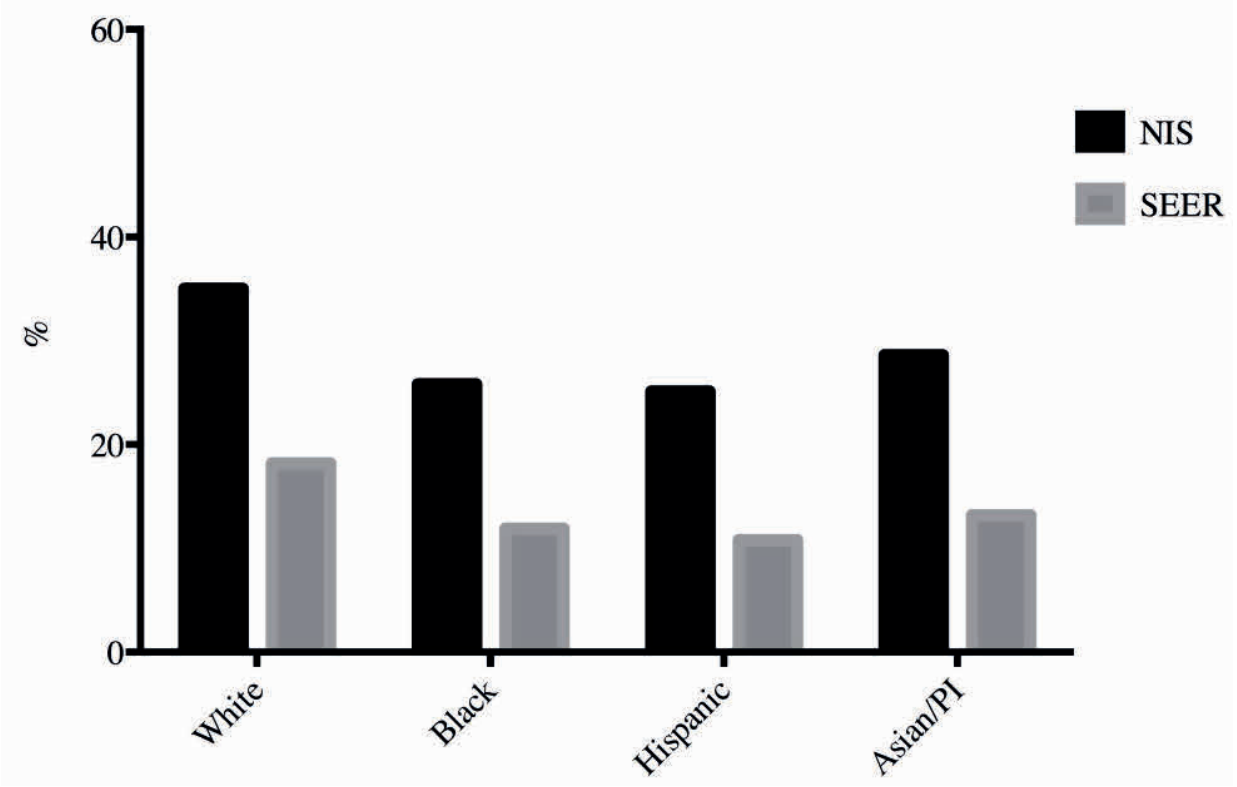

Figure 4a. Immediate breast reconstruction in invasive breast cancer patients in the state of California. 


\section{California: Immediate breast reconstruction in DCIS patients}

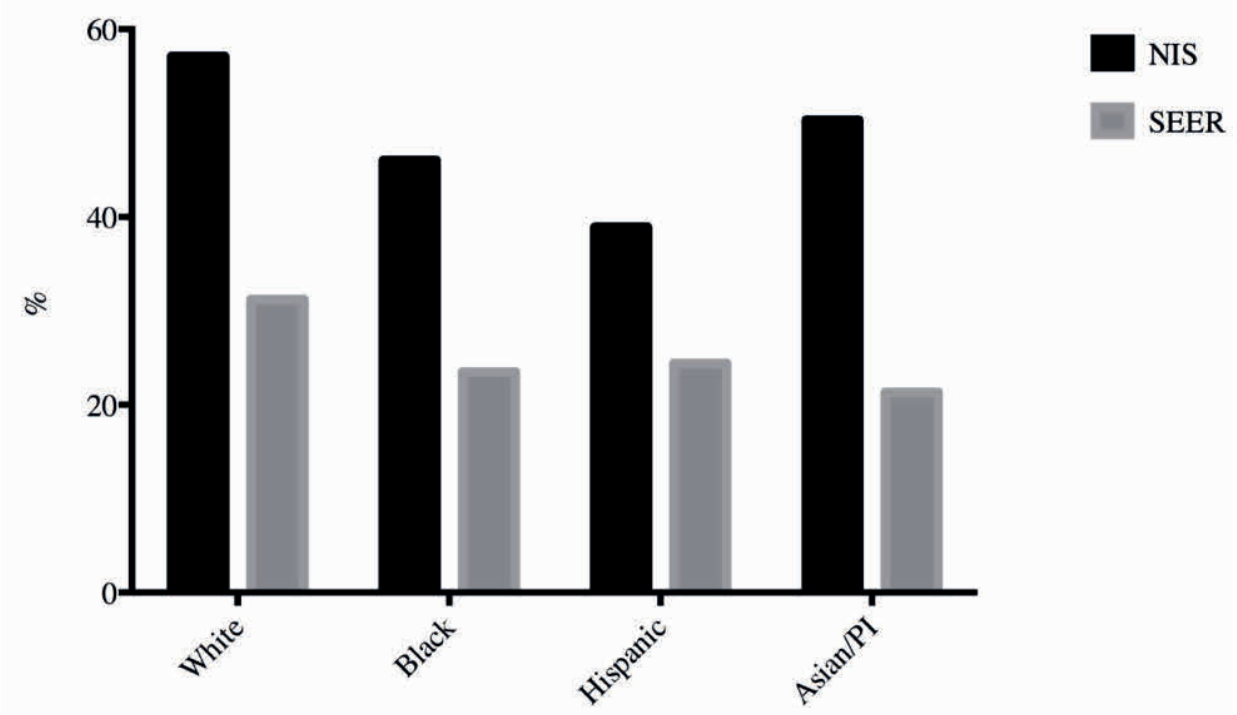

Figure 4b. Immediate breast reconstruction in DCIS patients in the state of California.

\section{DISCUSSION}

In this study, we present a nationwide comparison of immediate breast reconstruction as reported by three large national databases, the NIS, NSQIP and SEER. This study found, that despite large number of patients undergoing breast reconstruction in all databases, the reported frequency differed significantly for both invasive breast cancer patients and DCIS among databases. In both disease cohorts, a significant upward trend over time was seen in the utilization of immediate breast reconstruction in the NSQIP, NIS, and SEER (Figure 2a-b) $(p<0.001)$. Additionally, following sub-group analysis, the extent of disparities in immediate reconstruction differed significantly among databases (figure 3a-b).

The overall rate of immediate breast reconstruction following mastectomy in this study was $18.8 \%$ in invasive breast cancer patients within the SEER population, which is supported by the work of Ashfaq et $a l^{13}$, Agarwal et $a l^{5}$ and Lang et a ${ }^{30}$ who reported a $16 \%$ - 20\% immediate breast reconstruction rates using the same database. In the NIS cohort, we found immediate breast reconstruction in $32.7 \%$ invasive breast cancer patients and $51.4 \%$ of DCIS patients, similar to previous reports. ${ }^{6,7,23,37}$ Our study also found an increase in immediate breast reconstruction after mastectomy (from $11.5 \%$ to $47.0 \%$ in invasive breast cancer patients and from $23.2 \%$ to $66.4 \%$ in DCIS patients, 
between 1998 and 2012, respectively), which supports other studies using the NIS database. Albornoz et $a{ }^{6}$ demonstrated an increase in utilization of immediate breast reconstruction from $20.8 \%$ in 1998 to $37.8 \%$ in 2008 for patients diagnosed with breast cancer or increased risk of breast cancer. Other studies reflect similar rates of immediate breast reconstruction utilization after mastectomy ranging from $18.0 \%$ $38.0 \%$ 7,34,37,39 Finally, we found immediate reconstruction rates of $40.7 \%$ among invasive breast cancer patients and $54.1 \%$ among DCIS patients within the NSQIP population. A recent report by Kwok et al. and Butler et al. both reported a significant increase in immediate breast reconstruction utilization with rates similar to those found in our study (26.0\% - 29.7\% to 40.0\%- 42.8\% between 2005 and 2011/2012, respectively)). ${ }^{17,29}$

Despite the passage of the Women's Health and Cancer Right Act of 1998, many have noted women of color to be significantly less likely to receive breast reconstruction. 5, 7,9,10,12,17,26,27,30,32,38,39,44 As a result, the "Breast Cancer Patient Education Act of 2015" was introduced in 2015, in the effort to raise awareness about the availability and coverage of breast reconstruction, especially for racial and ethnic minority groups. ${ }^{45}$ This bill referred statistics provided by the American Cancer Society, multiple institutional studies and in terms of one large database, only SEER studies. ${ }^{45}$

Similar to previous studies, we found significant racial disparities in immediate breast reconstruction utilization. Our study, however, contributes new insight by and identifies significant interdatabase variability of racial disparities in immediate breast reconstruction. Our results showed that the rates of immediate breast reconstruction utilization differed among the different databases (figure 3a-b). This persisted even following sub-group analysis of the state of California, where variability in racial disparities was seen with differences up to $50 \%$ (Figure $4 a-b$ ).

Major differences in type and nature of the databases exist (Table 2). Generally, large-volume databases can be broadly categorized as either administrative or clinical based databases. Administrative databases, such as the HCUP (which includes the NIS, the Kids Inpatient Database (KID), the State Inpatient Database (SID) and State Ambulatory Surgery Database (SASD)), Medicare and The University Health Consortium (UHC) databases were originally not designed for clinical research, but rather to track billing for hospitals, providers, and procedures. Although providing a wealth of information, patient-level clinical information is not available in these administrative databases (stage of disease, tumor characteristics, preoperative performance status). Clinical databases, such as the NSQIP, SEER, National Cancer Database (NCDB), are developed with specific clinical goals in mind, and therefore populated with defined patient information. Additionally, the population within the database may be limited by specific restrictions include age (Medicare), or diagnosis (cancer registries).

The NSQIP, NIS, and the SEER are extensively utilized as nationwide representation of the U.S. population, but major variations exist in the states covered by the different databases (Figure 1a-c). Most importantly, it should be noted that NIS and NSQIP are 
reflect practice patterns in 47 or more states, while SEER reflects the practice of only 17. Previous studies focused on cancer care found treatment trends and outcomes varied according to volumes, teaching or academic medical centers, national cancer institute cancer centers designation, and subspecialist providers. ${ }^{50}$ When our study evaluated nationwide and $\mathrm{NCl}$ Cancer Centers coverage of the NSQIP, NIS and SEER databases we found that NSQIP and NIS cover the majority of states and $\mathrm{NCl}$ cancer hospitals. The SEER database however, covers only 18 cancer registries in 15 states and lacks coverage in most states and of the major cancer centers throughout the Unites States. Additionally, we showed that when comparing the databases in only one state, California, significant differences existed in immediate breast reconstruction utilization between the NIS and SEER (Figure 4a-b).

Sampling of the type of hospital is another factor that may also affect the rates of immediate breast reconstruction, with academic centers more likely to perform autologous reconstruction and immediate reconstruction. $7,23,25,51,52$ While SEER samples the population based on race, income, and education, with the specific aim of increasing generalizability to the United States population, it lacks coverage of the most major cancer centers throughout the United States including most major academic medical centers (Figure 1a, Figure 2). In the NSQIP, academic institutions are represented more prominently, potentially explaining the high immediate breast reconstruction rates when compared to the NIS and SEER. The NIS is a random sampling which may be the best representation, but remains only sampling through 2011.

There are important clinical implications to this research. Clinicians and researchers should have a basic understanding and should know the strengths and weaknesses of the databases in order to interpret the data. One database should therefore not uniformly be utilized for clinical guidelines and policies. This study has several notable limitations. First, all data is collected from national databases which are subject to missing data, coding errors, and poor long term follow-up. Additionally, this study is not able to assess long-term survival as NSQIP does not capture postoperative events occurring beyond 30 days, NIS includes only data from inpatient stay, and SEER does not include data beyond 4 months. However, Tseng et $\mathrm{al}^{53}$ revealed that only $8 \%$ of mastectomy patients pursued reconstruction in a delayed fashion, suggesting that the racial disparities in immediate breast reconstruction utilization revealed in this present study would be unlikely to significantly change if the delayed reconstruction patients were able to be captured. Additionally, the lowest rate of immediate breast reconstruction occurred in SEER, which interestingly also has the longest follow-up when compared to NSQIP and NIS. Finally, we are unable to account for patient preferences, which may vary across cultural groups; this is an important area for future research. 


\section{CONCLUSION}

Our study confirmed previous work suggesting that the utilization of immediate breast reconstruction has increased over time, and significant racial disparities exist. However, the reported rates of reconstruction are lowest and disparities are highest in the SEER database. These findings suggest that utilization of SEER may not be universally generalizable to the entire US population, and should be considered when studies are utilized for wider policy making.

\section{TABLE AND FIGURE LEGEND}

Figure 1a. Geographic map of the states included in the SEER database.

Figure 1b. Geographic map of the states included in the NIS database.

Figure 1c. Geographic map of the states included in the NSQIP database.

Figure 2a. Trend over time; Immediate breast reconstruction in invasive breast cancer patients.

*Cochrane-Armitage test for trend was $p<0.001$ in NIS, NSQIP and SEER.

Figure $2 \mathrm{~b}$. Trend over time; Immediate breast reconstruction in DCIS patients.

*Cochrane-Armitage test for trend was $p<0.001$ in NIS, NSQIP and SEER.

Figure 3a. Immediate breast reconstruction in invasive breast cancer patients stratified per race

Figure 3b. Immediate breast reconstruction in DCIS patients stratified per race

Figure 4a. Immediate breast reconstruction in invasive breast cancer patients in the state of California.

Figure 4b. Immediate breast reconstruction in DCIS patients in the state of California.

Table 1. Patient characteristics in invasive breast cancer and DCIS patients.

Table 2. Overview strengths and weaknesses NIS, NSQIP and SEER database.

Table, Supplemental digital content 1. International Classification of Diseases, Ninth Edition (ICD-9) breast cancer and carcinoma in situ coding.

Table, Supplemental digital content 2. ICD-9, CPT and Procedure mastectomy codes.

Table, Supplemental digital content 3. ICD-9, CPT and Procedure immediate breast reconstruction codes. 


\section{SUPPLEMENTAL DIGITAL CONTENT}

Table, Supplemental digital content 1. International Classification of Diseases, Ninth Edition (ICD-9) breast cancer and carcinoma in situ coding.

\begin{tabular}{ll}
\hline ICD-9 code & Description breast cancer and increased risk of breast cancer \\
\hline 174.0 & Malignant neoplasm of female breast: nipple and areola \\
174.1 & Malignant neoplasm of female breast: central portion \\
174.2 & Malignant neoplasm of female breast: upper inner-quadrant \\
174.3 & Malignant neoplasm of female breast: lower-inner quadrant \\
174.4 & Malignant neoplasm of female breast: upper-outer quadrant \\
174.5 & Malignant neoplasm of female breast: lower-outer quadrant \\
174.6 & Malignant neoplasm of female breast: axillary tail \\
174.8 & Malignant neoplasm of female breast: other sites \\
174.9 & Malignant neoplasm of female breast: unspecified \\
233.0 & Carcinoma in situ of breast \\
\hline
\end{tabular}

Table, Supplemental digital content 2. ICD-9, CPT and Procedure mastectomy codes.

\begin{tabular}{|c|c|}
\hline \multicolumn{2}{|c|}{ Nationwide Inpatient Sample (NIS) database inclusion criteria } \\
\hline ICD-9 code & Description Mastectomy \\
\hline 85.40 & Mastectomy \\
\hline 85.34 & Other unilateral subcutaneous mammectomy \\
\hline 85.41 & Unilateral simple mastectomy \\
\hline 85.43 & Unilateral extended simple mastectomy \\
\hline 85.45 & Unilateral radical mastectomy \\
\hline 85.47 & Unilateral extended radical mastectomy \\
\hline 85.33 & Unilateral subcutaneous mammectomy with synchronous implant \\
\hline 85.36 & Other bilateral subcutaneous mammectomy \\
\hline 85.42 & Bilateral simple mastectomy \\
\hline 85.44 & Bilateral extended simple mastectomy \\
\hline 85.46 & Bilateral radical mastectomy \\
\hline 85.48 & Bilateral extended radical mastectomy \\
\hline 85.35 & Bilateral subcutaneous mammectomy with synchronous implant \\
\hline \multicolumn{2}{|c|}{ National Surgical Quality Improvement Project (NSQIP) } \\
\hline CPT code & Description \\
\hline 19303 & Mastectomy, simple, complete \\
\hline 19304 & Mastectomy, subcutaneous \\
\hline 19305 & Mastectomy, radical, including pectoral muscles, axillary lymph nodes \\
\hline 19306 & $\begin{array}{l}\text { Mastectomy, radical, including pectoral muscles, axillary and internal mammary } \\
\text { lymph nodes (urban type operation) }\end{array}$ \\
\hline 19307 & $\begin{array}{l}\text { Mastectomy, modified radical, including axillary lymph nodes, with or without } \\
\text { pectoralis minor muscle, but excluding pectoralis major muscle. }\end{array}$ \\
\hline 19180 & Simple complete mastectomy \\
\hline 19182 & Subcutaneous mastectomy \\
\hline
\end{tabular}




\begin{tabular}{|c|c|}
\hline 19200 & Radical mastectomy \\
\hline 19220 & Urban type mastectomy \\
\hline 19240 & Modified radical mastectomy \\
\hline \multicolumn{2}{|c|}{ The Surveillance, Epidemiology and End Result (SEER) } \\
\hline Procedure coding & Description \\
\hline 40 & Simple mastectomy, NOS \\
\hline 41 & Simple mastectomy, NOS, Unilateral \\
\hline 43 & Simple mastectomy, NOS, Unilateral, reconstruction NOS \\
\hline 44 & Simple mastectomy, NOS, Unilateral, tissue reconstruction \\
\hline 45 & Simple mastectomy, NOS, Unilateral, implant reconstruction \\
\hline 46 & Simple mastectomy, NOS, Unilateral, combined reconstruction \\
\hline 42 & Simple mastectomy, NOS, Bilateral \\
\hline 47 & Simple mastectomy, NOS, Bilateral, reconstruction NOS \\
\hline 48 & Simple mastectomy, NOS, Bilateral, tissue Simple mastectomy, NOS, reconstruction \\
\hline 49 & Simple mastectomy, NOS, Bilateral, implant reconstruction \\
\hline 75 & Simple mastectomy, NOS, Bilateral, combined reconstruction \\
\hline 40 & Simple mastectomy, NOS \\
\hline 41 & Simple mastectomy, NOS, Unilateral \\
\hline 43 & Simple mastectomy, NOS, Unilateral, reconstruction NOS \\
\hline 44 & Simple mastectomy, NOS, Unilateral, tissue reconstruction \\
\hline 45 & Simple mastectomy, NOS, Unilateral, implant reconstruction \\
\hline 46 & Simple mastectomy, NOS, Unilateral, combined reconstruction \\
\hline 50 & Modified radical mastectomy \\
\hline 51 & Modified radical mastectomy, Unilateral \\
\hline 53 & Modified radical mastectomy, Unilateral, reconstruction NOS \\
\hline 54 & Modified radical mastectomy, Unilateral, tissue reconstruction \\
\hline 55 & Modified radical mastectomy, Unilateral, implant reconstruction \\
\hline 56 & Modified radical mastectomy, Unilateral, combined reconstruction \\
\hline 52 & Modified radical mastectomy, Bilateral \\
\hline 57 & Modified radical mastectomy, Bilateral, reconstruction NOS \\
\hline 58 & Modified radical mastectomy, Bilateral, tissue reconstruction \\
\hline 59 & Modified radical mastectomy, Bilateral, implant reconstruction \\
\hline 63 & Modified radical mastectomy, Bilateral, combined reconstruction \\
\hline 60 & Radical mastectomy, NOS \\
\hline 61 & Radical mastectomy, Unilateral \\
\hline 64 & Radical mastectomy, Unilateral, reconstruction NOS \\
\hline 65 & Radical mastectomy, Unilateral, tissue reconstruction \\
\hline 66 & Radical mastectomy, Unilateral, implant reconstruction \\
\hline 67 & Radical mastectomy, Unilateral, combined reconstruction \\
\hline 62 & Radical mastectomy, Bilateral \\
\hline 68 & Radical mastectomy, Bilateral, reconstruction NOS \\
\hline 69 & Radical mastectomy, Bilateral, tissue reconstruction \\
\hline 73 & Radical mastectomy, Bilateral, implant reconstruction \\
\hline
\end{tabular}




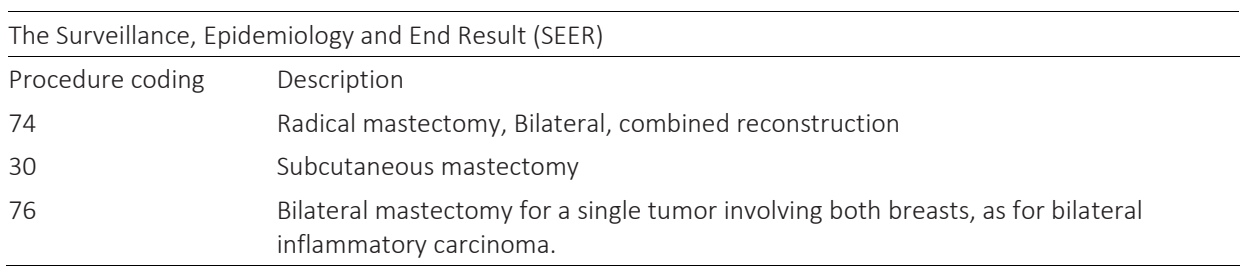

Table, Supplemental digital content 3. ICD-9, CPT and Procedure immediate breast reconstruction codes.

Nationwide Inpatient Sample (NIS) database inclusion criteria

ICD-9 code Description IBR

Implant-based breast reconstruction

85.33 Unilateral subcutaneous mammectomy with synchronous implant

$85.35 \quad$ Bilateral subcutaneous mammectomy with synchronous implant

$85.53 \quad$ Unilateral breast implant

$85.54 \quad$ Bilateral breast implant

$85.95 \quad$ Insertion breast tissue expander

85.96 Removal breast tissue expander

$85.84 \quad$ Breast pedicle graft

$85.85 \quad$ Breast muscle graft

\section{Autologous breast reconstruction}

$85.71 \quad$ Latissimus dorsi myocutaneous flap (LD), pedicled

85.72 Transverse rectus abdominis myocutaneous (TRAM) flap, pedicled

85.73 Transverse rectus abdominis myocutaneous (TRAM) flap, free

85.74 Deep inferior epigastric artery perforator (DIEP) flap, free

85.75 Superficial inferior epigastric artery (SIEA) flap, free

85.76 Gluteal artery perforator (GAP) flap, free

National Surgical Quality Improvement Project (NSQIP)

CPT code Description IBR

Implant-based breast reconstruction

$19340 \quad$ Immediate insertion of breast prosthesis following mastopexy, mastectomy or in reconstruction

19342 Delayed insertion of breast prosthesis following mastoplexy, mastectomy, or in reconstruction

19357 Breast reconstruction, immediate or delayed, with tissue expander, including subsequent expansion

Autologous breast reconstruction

Breast reconstruction with latissimus dorsi flap, without prosthetic implant

Breast reconstruction with free flap

19368 Breast reconstruction with transverse rectus abdominis myocutaneous flap (TRAM), single pedicle, including closure of donor site; with microvascular anastomosis (supercharging)

19369 Breast reconstruction with transverse rectus abdominis myocutaneous flap (TRAM, double pedicle, including closure of donor site) 
The Surveillance, Epidemiology and End Result (SEER)

Procedure coding

45

49

55

59

66

73

44

48

54

58

65

69

46

75

56

63

67

74
Description IBR

Implant-based breast reconstruction

Simple mastectomy, NOS, Unilateral, implant reconstruction

Simple mastectomy, NOS, Bilateral, implant reconstruction

Modified radical mastectomy, Unilateral, implant reconstruction

Modified radical mastectomy, Bilateral, implant reconstruction

Radical mastectomy, Unilateral, implant reconstruction

Radical mastectomy, Bilateral, implant reconstruction

Autologous breast reconstruction

Simple mastectomy, NOS, Unilateral, tissue reconstruction

Simple mastectomy, NOS, Bilateral, tissue reconstruction

Modified radical mastectomy, Unilateral, tissue reconstruction

Modified radical mastectomy, Bilateral, tissue reconstruction

Radical mastectomy, Unilateral, tissue reconstruction

Radical mastectomy, Bilateral, tissue reconstruction

Combined implant-based and autologous breast reconstruction

Simple mastectomy, NOS, Unilateral, combined reconstruction

Simple mastectomy, NOS, Bilateral, combined reconstruction

Modified radical mastectomy, Unilateral, combined reconstruction

Modified radical mastectomy, Bilateral, combined reconstruction

Radical mastectomy, Unilateral, combined reconstruction

Radical mastectomy, Bilateral, combined reconstruction 


\section{REFERENCES}

1. National Cancer Institute, Surveillance Epidemiology, and End Result (SEER) data description. Available at: http://seer.cancer.gov/data/ Assessed: 1 Dec. 2015.

2. The American College of Surgeons National Surgical Quality Improvement Program (ACS NSQIP) Available at: https://www.facs.org/quality-programs/acs-nsqip Assessed: 15 December 2015.

3. Healthcare Cost and Utilization Project (HCUP) overview of the Nationwide Inpatient Sample (NIS) database. Available at: http://www.hcup-us.ahrq.gov/nisoverview.jsp Assessed: 1 December 2015.

4. Agarwal, S., Kidwell, K. M., Farberg, A., Kozlow, J. H., Chung, K. C., Momoh, A. O. Immediate Reconstruction of the Radiated Breast: Recent Trends Contrary to Traditional Standards. Annals of surgical oncology 2015;22:2551-2559.

5. Agarwal, S., Pappas, L., Neumayer, L., Agarwal, J. An analysis of immediate postmastectomy breast reconstruction frequency using the surveillance, epidemiology, and end results database. The breast journal 2011;17:352-358.

6. Albornoz, C. R., Bach, P. B., Mehrara, B. J., et al. A paradigm shift in U.S. Breast reconstruction: increasing implant rates. Plastic and reconstructive surgery 2013;131:15-23.

7. Albornoz, C. R., Bach, P. B., Pusic, A. L., et al. The influence of sociodemographic factors and hospital characteristics on the method of breast reconstruction, including microsurgery: a U.S. population-based study. Plastic and reconstructive surgery 2012;129:1071-1079.

8. Albornoz, C. R., Cordeiro, P. G., Mehrara, B. J., et al. Economic implications of recent trends in U.S. immediate autologous breast reconstruction. Plastic and reconstructive surgery 2014;133:463-470.

9. Alderman, A. K., Hawley, S. T., Janz, N. K., et al. Racial and ethnic disparities in the use of postmastectomy breast reconstruction: results from a population- based study. Journal of clinical oncology : official journal of the American Society of Clinical Oncology 2009;27:5325-5330.

10. Alderman, A. K., McMahon, L., Jr., Wilkins, E. G. The national utilization of immediate and early delayed breast reconstruction and the effect of sociodemographic factors. Plastic and reconstructive surgery 2003;111:695-703; discussion 704-695.

11. Alderman, A. K., Wei, Y., Birkmeyer, J. D. Use of breast reconstruction after mastectomy following the Women's Health and Cancer Rights Act. Jama 2006;295:387-388.

12. Amaral, M. H., Dao, H., Shin, J. H. Racial and socioeconomic disparities in reduction mammoplasty: an analysis of nationwide inpatient sample database. Annals of plastic surgery 2011;66:476-478.

13. Ashfaq, A., McGhan, L. J., Pockaj, B. A., et al. Impact of breast reconstruction on the decision to undergo contralateral prophylactic mastectomy. Annals of surgical oncology 2014;21:2934-2940.

14. Baxter, N. N., Virnig, B. A., Durham, S. B., Tuttle, T. M. Trends in the treatment of ductal carcinoma in situ of the breast. Journal of the National Cancer Institute 2004;96:443-448.

15. Bezuhly, M., Temple, C., Sigurdson, L. J., Davis, R. B., Flowerdew, G., Cook, E. F., Jr. Immediate postmastectomy reconstruction is associated with improved breast cancer-specific survival: evidence and new challenges from the Surveillance, Epidemiology, and End Results database. Cancer 2009;115:4648-4654.

16. Blaschko, S. D., Harris, C. R., Zaid, U. B., et al. Trends, utilization, and immediate perioperative complications of urethroplasty in the United States: data from the national inpatient sample 2000-2010. Urology 2015;85:1190-1194.

17. Butler, P. D., Nelson, J. A., Fischer, J. P., et al. Racial and age disparities persist in immediate breast reconstruction: an updated analysis of 48,564 patients from the 2005 to 2011 American College of Surgeons National Surgery Quality Improvement Program data sets. American journal of surgery 2015.

18. Cemal, Y., Albornoz, C. R., Disa, J. J., et al. A paradigm shift in U.S. breast reconstruction: Part 2. The influence of changing mastectomy patterns on reconstructive rate and method. Plastic and reconstructive surgery 2013;131:320e-326e.

19. Chow, I., Hanwright, P. J., Hansen, N. M., Leilabadi, S. N., Kim, J. Y. Predictors of 30-day readmission after mastectomy: A multi-institutional analysis of 21,271 patients. Breast disease 2015;35:221-231. 
20. Coleman, E. A., Kessler, L. G., Wun, L. M., Feuer, E. J. Trends in the surgical treatment of ductal carcinoma in situ of the breast. American journal of surgery 1992;164:74-76.

21. Fine, N. A., Hirsch, E. M. Keeping options open for patients with anticipated postmastectomy chest wall irradiation: immediate tissue expansion followed by reconstruction of choice. Plastic and reconstructive surgery 2009;123:25-29.

22. Greenberg, C. C., Schneider, E. C., Lipsitz, S. R., et al. Do variations in provider discussions explain socioeconomic disparities in postmastectomy breast reconstruction? Journal of the American College of Surgeons 2008;206:605-615.

23. Habermann, E. B., Thomsen, K. M., Hieken, T. J., Boughey, J. C. Impact of availability of immediate breast reconstruction on bilateral mastectomy rates for breast cancer across the United States: data from the nationwide inpatient sample. Annals of surgical oncology 2014;21:3290-3296.

24. Hernandez-Boussard, T., Zeidler, K., Barzin, A., Lee, G., Curtin, C. Breast reconstruction national trends and healthcare implications. The breast journal 2013;19:463-469.

25. In, H., Jiang, W., Lipsitz, S. R., Neville, B. A., Weeks, J. C., Greenberg, C. C. Variation in the utilization of reconstruction following mastectomy in elderly women. Annals of surgical oncology 2013;20:1872-1879.

26. Innos, K., Horn-Ross, P. L. Recent trends and racial/ethnic differences in the incidence and treatment of ductal carcinoma in situ of the breast in California women. Cancer 2003;97:1099-1106.

27. Kotwall, C., Brinker, C., Covington, D., et al. Local and national trends over a decade in the surgical treatment of ductal carcinoma in situ. American journal of surgery 2003;186:723-728; discussion 728-729.

28. Kummerow, K. L., Du, L., Penson, D. F., Shyr, Y., Hooks, M. A. Nationwide trends in mastectomy for earlystage breast cancer. JAMA surgery 2015;150:9-16.

29. Kwok, A. C., Goodwin, I. A., Ying, J., Agarwal, J. P. National trends and complication rates after bilateral mastectomy and immediate breast reconstruction from 2005 to 2012. American journal of surgery 2015;210:512-516.

30. Lang, J. E., Summers, D. E., Cui, H., et al. Trends in post-mastectomy reconstruction: a SEER database analysis. Journal of surgical oncology 2013;108:163-168.

31. Lucas, D. J., Sabino, J., Shriver, C. D., Pawlik, T. M., Singh, D. P., Vertrees, A. E. Doing more: trends in breast cancer surgery, 2005 to 2011. The American surgeon 2015;81:74-80.

32. Mahmoudi, E., Giladi, A. M., Wu, L., Chung, K. C. Effect of federal and state policy changes on racial/ethnic variation in immediate postmastectomy breast reconstruction. Plastic and reconstructive surgery 2015;135:1285-1294.

33. Morrow, M. National treatment trends for ductal carcinoma in situ of the breast. Arch Surg 1997 Jun;132:660-665. Journal of women's health / the official publication of the Society for the Advancement of Women's Health Research 1998;7:267-268.

34. Reuben, B. C., Manwaring, J., Neumayer, L. A. Recent trends and predictors in immediate breast reconstruction after mastectomy in the United States. American journal of surgery 2009;198:237-243.

35. Sanati-Mehrizy, P., Massenburg, B. B., Rozehnal, J. M., et al. A Comparison of Postoperative Outcomes in Immediate Versus Delayed Reconstruction After Mastectomy. Eplasty 2015;15:e44.

36. Tseng, W. H., Stevenson, T. R., Canter, R. J., et al. Sacramento area breast cancer epidemiology study: use of postmastectomy breast reconstruction along the rural-to-urban continuum. Plastic and reconstructive surgery 2010;126:1815-1824.

37. Wexelman, B., Schwartz, J. A., Lee, D., Estabrook, A., Ma, A. M. Socioeconomic and geographic differences in immediate reconstruction after mastectomy in the United States. The breast journal 2014;20:339-346.

38. Wilkins, E. G., Alderman, A. K. Breast reconstruction practices in north america: current trends and future priorities. Seminars in plastic surgery 2004;18:149-155.

39. Yang, R. L., Newman, A. S., Lin, I. C., et al. Trends in immediate breast reconstruction across insurance groups after enactment of breast cancer legislation. Cancer 2013;119:2462-2468.

40. Malay, S., Shauver, M. J., Chung, K. C. Applicability of large databases in outcomes research. The Journal of hand surgery 2012;37:1437-1446. 
41. Chung, K. C., Swanson, J. A., Schmitz, D., Sullivan, D., Rohrich, R. J. Introducing evidence-based medicine to plastic and reconstructive surgery. Plastic and reconstructive surgery 2009;123:1385-1389.

42. American Society of Plastic Surgeons (ASPS). Evidence-based Clinical Practice Guidelines: Breast reconstruction with Expanders and Implants. Available at: http://www.plasticsurgery.org/Documents/ Health-Policy/Guidelines/guideline-2013-breast-recon-expanders-implants.pdf Assessed: 2 february 2016.

43. Shippee, T. P., Kozhimannil, K. B., Rowan, K., Virnig, B. A. Health insurance coverage and racial disparities in breast reconstruction after mastectomy. Women's health issues : official publication of the Jacobs Institute of Women's Health 2014;24:e261-269.

44. Offodile, A. C., 2nd, Tsai, T. C., Wenger, J. B., Guo, L. Racial disparities in the type of postmastectomy reconstruction chosen. The Journal of surgical research 2015;195:368-376.

45. Breast Cancer Patient Education Act fo 2015. Available at: https://www.congress.gov/bill/114thcongress/senate-bill/1192/text Assessed: February 3rd, 2016.

46. National Cancer Institute Designated Cancer Centers. Available at: http://www.cancer.gov/research/ncirole/cancer-centers. Assessed 2 February 2016.

47. Nationwide Inpatient Sample (NIS) Data elements. Available at: http://www.hcup-us.ahrq.gov/db/vars/ _prn/nisnote.jsp Assessed February 1st 2016.

48. ACS NSQIP Data Elements. Available at: https://www.facs.org/ /media/files/quality programs/nsqip/ nsqip_puf_userguide_2014.ashx. Assessed February 1st 2016.

49. National Cancer Institute Surveillance, Epidemiology and End Result (SEER) Data Elements. Available at: http://seer.cancer.gov/data/documentation.html Assessed February 1st 2016.

50. Onega, T., Duell, E. J., Shi, X., Demidenko, E., Goodman, D. C. Race versus place of service in mortality among medicare beneficiaries with cancer. Cancer 2010;116:2698-2706.

51. Onega, T., Weiss, J., Kerlikowske, K., et al. The influence of race/ethnicity and place of service on breast reconstruction for Medicare beneficiaries with mastectomy. SpringerPlus 2014;3:416.

52. Masoomi, H., Wirth, G. A., Paydar, K. Z., Richland, B. K., Evans, G. R. Perioperative outcomes of autologous breast reconstruction surgery in teaching versus nonteaching hospitals. Plastic and reconstructive surgery 2014;134:514e-520e.

53. Tseng, J. F., Kronowitz, S. J., Sun, C. C., et al. The effect of ethnicity on immediate reconstruction rates after mastectomy for breast cancer. Cancer 2004;101:1514-1523. 


\section{Chapter}

\section{Immediate Breast Reconstruction in the Netherlands and the United States: a Proof of Concept to Internationally Compare Quality of Care Using Cancer Registry Data}

Parisa Kamali

Annelotte van Bommel

Babette Becherer

Rodney Cooter

Marc A.M. Mureau

Andrea Pusic

Sabine Siesling

René R.J.W. van der Hulst

Samuel J. Lin

Hinne A. Rakhorst 


\section{ABSTRACT}

\section{Introduction}

Studies based large-volume databases have made significant contributions to research on breast cancer (BC) surgery. To date, no comparison between large-volume databases has been made internationally. This is the first proof of concept study exploring the feasibility of combining two existing operational databases of the Netherlands and the United States, focusing on BC care and immediate breast reconstruction (IBR) specifically.

\section{Methods}

The Netherlands Breast Cancer Audit (NBCA) (2011-2015) and the Surveillance, Epidemiology and End Result (SEER) database (2010-2013) were compared on structure and content. Data variables were grouped into general, treatment specific, cancer specific and follow-up variables and were matched. As proof of concept, mastectomy and IBR rates in patients diagnosed with invasive BC or ductal carcinoma in-situ (DCIS) were analyzed.

\section{Results}

The NBCA included 115 variables and the SEER 112. The NBCA included significantly more treatment-specific variables ( $n=46$ vs 6 ), while the SEER included more cancerspecific variables ( $n=74$ vs 26). In patients diagnosed with BC or DCIS, IBR was performed in $19.3 \%$ and $24.0 \%$ of the $B C$ cohort and $44.0 \%$ and $35.3 \%$ of the DCIS cohort in the NBCA and SEER, respectively. IBR rates increased significantly over time in both datasets.

\section{Conclusion}

This study provides a first overview of available registry data on BC care the Netherlands and the US and revealed limited data on treatment in the US. Comparison of treatment patterns of IBR showed interesting differences. Therefore, this study advocates the urgency for an international database with alignment of (treatment) variables in order to improve quality of $\mathrm{BC}$ care for all patients across the globe. 


\section{INTRODUCTION}

Studies based on large-volume (national) registries and other administrative datasets have made significant impact in the field of breast cancer treatment. ${ }^{1-10}$ Generally speaking, large-volume registries can be broadly categorized as either administrative or clinically based. These databases collectively offer unique opportunities to study patterns of care, guideline adherence, variation in practice, and outcomes following interventions. The ability to compare these data across populations and time points can facilitate new scientific discoveries, faster access to treatment interventions, and enable differences between populations to be delineated, allowing for identification of optimal treatment protocols and strategies to finally improve quality of care globally. ${ }^{11}$

Although there are a wide variety of breast cancer databases, the ability to compare data is limited by the heterogeneity of data elements, variable definitions, inclusion criteria, and coding systems in the registries. ${ }^{12}$ Direct comparisons of data variables between countries are rarely done because disease and treatment information contained in administrative databases cannot be aggregated into a single, searchable database. More recently, the scientific community has been increasingly advocated for the development of collaborative registries that facilitate high-quality data collection, harmonization, and analysis from clinical and research sites globally. ${ }^{12-19}$ Although some parameters in existing data sources can be compared, lack of early consensus on mutually useful treatment and outcome measures between data curators means that utility of much collected data is only seen locally. ${ }^{20}$ To date, there are only a few direct comparisons of quality of care and clinical outcomes at hospital level between various healthcare systems.

We undertook a proof of concept study to explore the feasibility of combining two large, operational databases in two countries and compare treatment patterns on immediate breast reconstruction (IBR) in women with early stage breast cancer. In these women, the primary treatment options are either mastectomy or breast conserving surgery with radiotherapy. ${ }^{21-25}$ For those opting for mastectomy, clinical guidelines recommend that breast reconstruction should be provided to all women, as it has been shown to significantly improve quality of life and body image. ${ }^{26-35}$ Yet, it is unknown whether this recommendation is being followed across the globe as the majority of studies report large variability in breast reconstruction rates both within and across countries. ${ }^{2,36-49}$

We present the first international comparison of IBR treatment patterns between the national Dutch breast cancer database (NABON (National Breast cancer Organization the Netherlands) Breast Cancer Audit (NBCA) and the most widely utilized, population-based tumor registry database of the United States (The Surveillance, Epidemiology and End Result (SEER)). The aims of this study were twofold: 1) to evaluate the comparability of the two databases, and, as an example to show the importance of the possibility of comparisons, 2) to evaluate the differences in IBR rates between both countries. 


\section{MATERIALS AND METHODS}

\section{Databases}

The NBCA and SEER database were utilized in this study for the Netherlands and the United States, respectively. At the time of this study, data were available for the years 2011 to 2015 for the NBCA and 2010 to 2013 for the SEER database. Due to the nature of the study, the authors decided to include all data in both databases, despite differences in the collection period.

The NBCA is a national multidisciplinary quality improvement audit developed in 2011 as part of the NABON. The registry contains data of all patients diagnosed with invasive breast cancer (BC) or ductal carcinoma in situ (DCIS) from all hospitals $(n=92)$ in the Netherlands. Hospitals can choose to collect data themselves or have datamanagers of the Netherlands Cancer Registry (NCR) register the data (the latter option being used by $75 \%$ of the hospitals). By providing feedback to the participating individual hospitals on their outcomes, the NBCA enables institutions to evaluate their data and start improvement projects. ${ }^{50,51}$ In 2015, the database included 32 quality indicators measuring the structure, processes, and outcomes of breast cancer care. ${ }^{51,52}$ For research, an official request must be made to the scientific committee of the NBCA and the NCR, who judge on relevance of the research question and privacy of the patients, care givers and institutions.

The SEER program of the National Cancer Institute $(\mathrm{NCl})$ is a population-based tumor registry designed to track cancer incidence, cancer directed surgery, and radiation therapy provided for the first course of treatment and survival in 18 cancer registries across 15 states. ${ }^{53}$ The SEER collects very broad patient characteristics and cancer specific information. ${ }^{54}$ The SEER data contains 135 collected variables, including the International Classification of Diseases (ICD-9) diagnosis codes and the SEER Program Coding and Staging for the procedures performed. SEER data are publicly available for studies of cancer-related epidemiology. After signing a Research Data Agreement, the data will be available for download or via DVD sent via US mail.

\section{Database comparison}

The NBCA dataset variables is provided with the following sections: general information, current episode, clinical examination, diagnostics used and multidisciplinary approach, surgical procedure including re-excision, pathology, radiation therapy, systemic therapy, and follow-up. Variables are provided in Dutch and were translated in English. Descriptive terms for variables were utilized and subsequent discussion followed between the authors to confirm interpretation of variable descriptors. The SEER database variables were divided into different sections including: information source, demographic information, description of neoplasm, stage of disease at diagnosis, data collection system, first course of therapy, follow-up information and administrative 
codes. The variables of the NBCA dataset and the SEER dataset were manually grouped into the following sections; general, treatment specific, cancer specific and followup/other. Variables were matched in terms of data definitions.

\section{Proof of concept on differences in mastectomy and IBR rates}

All women aged $\geq 18$ years old and diagnosed with invasive breast cancer (BC) or Ductal Carcinoma In Situ (DCIS) undergoing mastectomy or immediate breast reconstruction were identified and included in this study. Patients under the age of 18, male patients, those undergoing outpatient procedures, and those with unknown age or gender were excluded. Mastectomy and IBR rates and trend over time were evaluated both for invasive breast cancer and DCIS patients and compared between the two countries.

\section{Statistical analysis}

Descriptive statistics are reported as proportions and compared using Pearson's Chisquare. Trends are analyzed using the Cochran-Armitage test for trend. All statistical analyses were performed using IBM SPSS version 22.0 (IBM Corp., Armonk, NY) and significance was set at $p<0.05$.

\section{RESULTS}

\section{Variables}

The NBCA file contained a total of 115 variables. The variables were divided into 24 general, 52 treatment specific, 27 cancer specific, and 12 follow-up variables. The SEER database contained a total of 135 data variables. Of these, 23 were historical variables that are no longer collected. These variables were excluded from the analysis. Of the remaining 112 variables, 23 were general, 5 treatment specific, 74 cancer specific, and 10 follow-up variables (Figure 1). Of the 115 variables available in the NBCA database and the 112 variables in the SEER database, a total of $8 \%(n=9)$ were matched and analyzed (Figure 1). The variables include general $(n=4,17 \%)$, treatment specific $(n=2$, $7.8 \%)$, and cancer specific $(n=3,6.1 \%)$ data. The matched variables included sex, diagnosis, type of reconstruction, age, laterality of tumor, radiation therapy, TNMclassification, tumor grade, receptor groups (Her2, HR, estrogen and progesterone receptor group). In both databases, four types of Immediate Breast Reconstruction (IBR) were defined: implant breast reconstruction (including tissue expander), autologous breast reconstruction, a combination of both, and reconstruction not otherwise specified following mastectomy. 


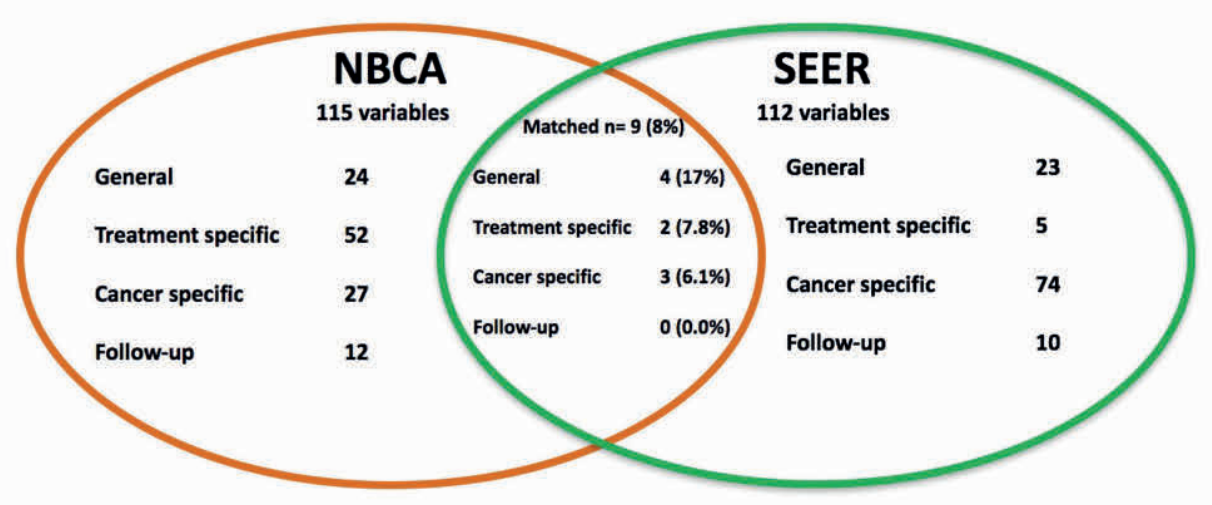

Figure 1. Overview matched variables NBCA and SEER

Examples of variables that did not match or were not available in one of the datasets included race, ethnicity, marital status at diagnosis, primary payer at diagnosis, (type of) chemotherapy, regional lymph node surgery, follow-up timeframe and vital status among others. Moreover, data on secondary or delayed reconstruction, if any, were not available. A vast number of variables included in the "cancer specific" section of the SEER database included variables that were recoded from earlier coding schemes (e.g. ICD-O-2 to ICD-O-3). The NBCA database included diagnostic variables such as MRI, BIRADS, multidisciplinary consultation which were not available in the SEER data.

Invasive breast cancer patients: mastectomy and IBR rates

In the NBCA database, a total of 61,988 patients diagnosed with breast cancer were identified and included. Of these, 24,361 (39.3\%) patients underwent mastectomy. The SEER dataset comprised a total of 213,914 breast cancer patients of whom 96,972 (42.0\%) underwent mastectomy. Within the NBCA cohort IBR mainly consisted of implant based reconstruction (90.6\%). Within the SEER cohort $46.6 \%$ underwent implant-based breast reconstruction and $36.6 \%$ underwent autologous breast reconstruction or a combined type of breast reconstruction (16.0\%).

Notably, in both cohorts, a decrease in mastectomy was seen over time (from $41.0 \%$ in 2011 to $37.6 \%$ in 2015 ( $p<0.001$ ) in the NBCA and from $43.1 \%$ in 2010 to $41.4 \%$ in $2013(p<0.001)$ in the SEER) (Figure 2a). Over time, an increase in IBR was also seen in both cohorts, (from $14.4 \%$ to $26.0 \%$ and from $21.7 \%$ to $26.6 \%$ in the NBCA and SEER cohort, $p<0.001$, respectively) (Figure $2 b$ ). Overall characteristics are presented in Table 1. The majority of patients undergoing breast reconstruction were aged 50-59 years old in the NBCA (34.4\%) cohort versus $40-49$ years in the SEER cohort (31.8\%). Approximately $73.1 \%$ of patients in the NBCA and $76.9 \%$ of the SEER population underwent radiation therapy. Similar rates were seen in terms of TNM-classification, grade of tumor, receptor groups, and estrogen and progesterone-receptor groups. 
Trend over time mastectomy in invasive breast cancer patients

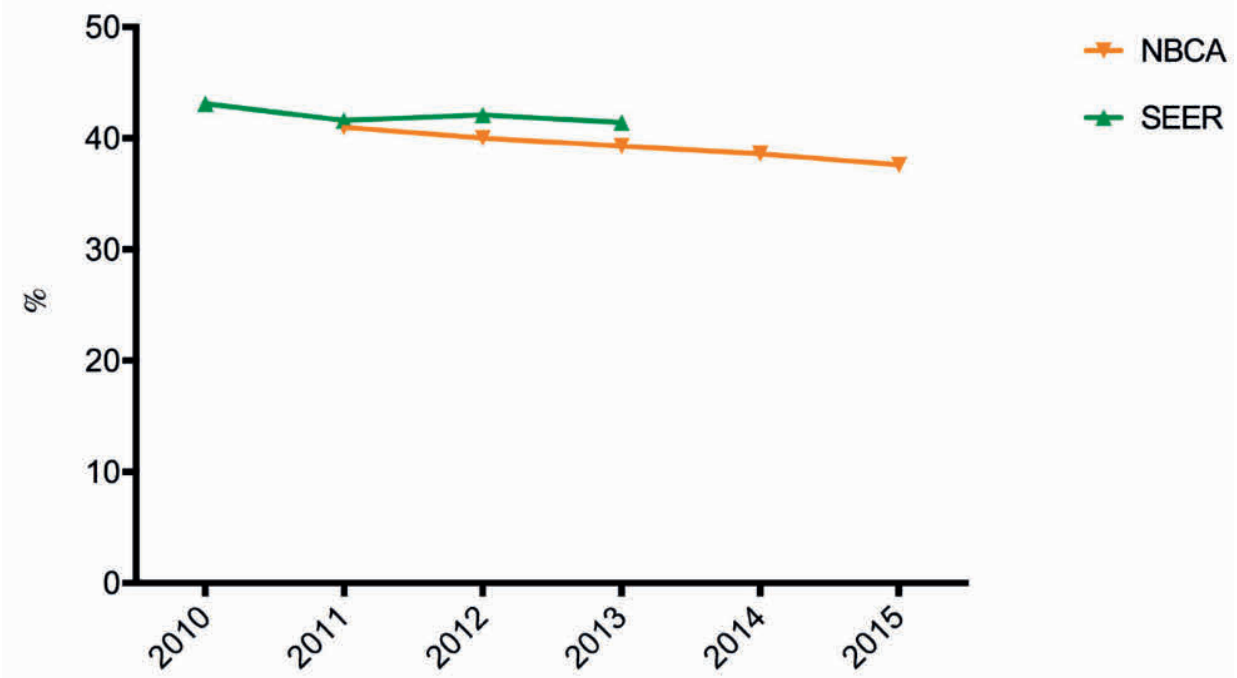

Figure 2a. Trend over time mastectomy in invasive breast cancer patients. Trend over time $p<0.001$

\section{Post-mastectomy immediate breast reconstruction in invasive breast cancer patients}

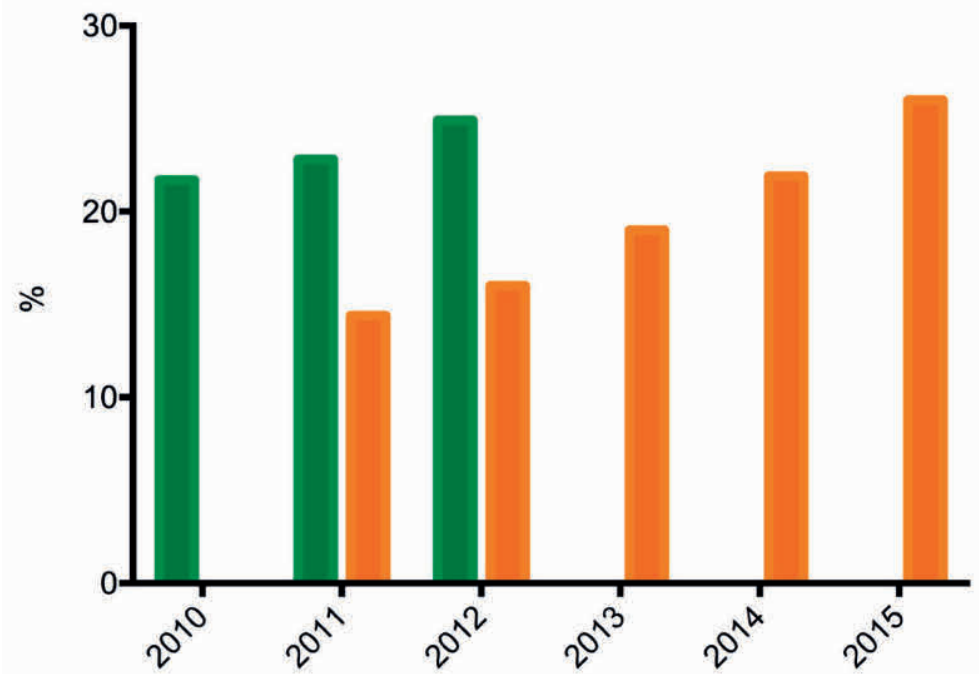

NBCA

SEER

Figure $2 \mathrm{~b}$. Trend over time immediate breast reconstruction in invasive breast cancer patients. Trend over time $p<0.001$ 
Chapter 9

Table 1. Overall characteristics invasive breast cancer and ductal carcinoma in situ patients

\begin{tabular}{|c|c|c|c|c|c|c|c|c|}
\hline & \multicolumn{4}{|c|}{ Invasive Breast Cancer } & \multicolumn{4}{|l|}{ DCIS } \\
\hline & \multicolumn{2}{|c|}{$\begin{array}{l}\text { NBCA } \\
2011-2015\end{array}$} & \multicolumn{2}{|c|}{$\begin{array}{l}\text { SEER } \\
2010-2013\end{array}$} & \multicolumn{2}{|c|}{$\begin{array}{l}\text { NBCA } \\
2011-2015\end{array}$} & \multicolumn{2}{|c|}{$\begin{array}{l}\text { SEER } \\
2010-2013\end{array}$} \\
\hline & No. & $(\%)$ & No. & $(\%)$ & No. & $(\%)$ & No. & $(\%)$ \\
\hline$N=$ & 61,988 & & 213,914 & & 9,886 & & 60,683 & \\
\hline IBR & 4,691 & $(19.3)$ & 23,302 & $(24.0)$ & 1,340 & $(44.0)$ & 6,960 & $(36.3)$ \\
\hline \multicolumn{9}{|l|}{$\mathrm{IBR}^{1,2}$} \\
\hline Implant & 4,205 & $(90.6)$ & 10,852 & $(46.6)$ & 1,146 & $(87.5)$ & 3,219 & $(46.3)$ \\
\hline Autologous & 236 & $(5.1)$ & 8,545 & $(36.6)$ & 80 & $(6.1)$ & 2,536 & $(36.4)$ \\
\hline Combined & 200 & $(4.3)$ & 3,905 & $(16.8)$ & 86 & $(6.6)$ & 1,205 & $(17.3)$ \\
\hline \multicolumn{9}{|l|}{ Age } \\
\hline$<39$ & 677 & $(14.4)$ & 2,766 & $(11.9)$ & 125 & $(9.3)$ & 458 & $(6.6)$ \\
\hline $40-49$ & 1,446 & $(30.8)$ & 7,298 & $(31.3)$ & 300 & $(22.4)$ & 2,521 & $(36.2)$ \\
\hline $50-59$ & 1,617 & $(34.4)$ & 7,254 & $(31.1)$ & 616 & $(46.0)$ & 2,309 & $(33.2)$ \\
\hline $60-69$ & 787 & $(16.8)$ & 4,671 & $(20.0)$ & 247 & $(18.4)$ & 1,363 & (19.6) \\
\hline$>70$ & 162 & (3.5) & 1,313 & $(5.6)$ & 52 & (3.9) & 309 & $(4.4)$ \\
\hline \multicolumn{9}{|c|}{ Laterality tumor } \\
\hline Left & 2,385 & $(50.8)$ & 11,659 & $(50.0)$ & 689 & $(51.4)$ & 3,520 & $(50.6)$ \\
\hline Right & 2,302 & $(49.2)$ & 11,632 & $(49.9)$ & 651 & $(48.6)$ & 3,440 & $(49.4)$ \\
\hline Unknown & & & 11 & $(0.1)$ & & & & \\
\hline \multicolumn{9}{|c|}{ Radiationtherapy $y^{3,4}$} \\
\hline no & 2,238 & $(73.1)$ & 17,651 & $(75.7)$ & & & & \\
\hline yes & 823 & $(26.9)$ & 5,651 & $(24.3)$ & & & & \\
\hline \multicolumn{9}{|c|}{ TNM classification } \\
\hline \multicolumn{9}{|l|}{ T-classification } \\
\hline $\mathrm{T} 1$ & 2,926 & $(62.4)$ & 13,133 & $(56.4)$ & & & & \\
\hline $\mathrm{T} 2$ & 1,244 & $(26.5)$ & 7,575 & $(32.5)$ & & & & \\
\hline $\mathrm{T} 3$ & 141 & $(3.0)$ & 1,832 & $(7.9)$ & & & & \\
\hline $\mathrm{T} 4$ & 12 & $(0.3)$ & 362 & $(1.6)$ & & & & \\
\hline Unknown & 362 & $(7.8)$ & 400 & (1.9) & & & & \\
\hline \multicolumn{9}{|c|}{ N-classification } \\
\hline NO & 3,172 & $(67.6)$ & 14,970 & $(64.2)$ & & & & \\
\hline N1 & 1,177 & $(25.1)$ & 6,099 & $(26.2)$ & & & & \\
\hline N2 & 162 & $(3.5)$ & 1,428 & $(6.1)$ & & & & \\
\hline N3 & 56 & $(1.2)$ & 721 & $(3.1)$ & & & & \\
\hline Unknown & 124 & $(2.6)$ & 84 & $(0.4)$ & & & & \\
\hline \multicolumn{9}{|l|}{ Grade } \\
\hline 1 & 898 & $(19.1)$ & 4,438 & $(19.0)$ & & & & \\
\hline 2 & 1,995 & $(42.6)$ & 9,917 & $(42.6)$ & & & & \\
\hline 3 & 1,260 & $(26.9)$ & 7,758 & (33.3) & & & & \\
\hline 4 & 0.0 & $(0.0)$ & 86 & $(0.4)$ & & & & \\
\hline Unknown & 538 & (11.4) & 1,103 & $(4.7)$ & & & & \\
\hline
\end{tabular}




\begin{tabular}{|c|c|c|c|c|c|c|c|c|}
\hline & \multicolumn{4}{|c|}{ Invasive Breast Cancer } & \multicolumn{4}{|l|}{ DCIS } \\
\hline & \multicolumn{2}{|c|}{$\begin{array}{l}\text { NBCA } \\
2011-2015\end{array}$} & \multicolumn{2}{|c|}{$\begin{array}{l}\text { SEER } \\
2010-2013\end{array}$} & \multicolumn{2}{|c|}{$\begin{array}{l}\text { NBCA } \\
2011-2015\end{array}$} & \multicolumn{2}{|c|}{$\begin{array}{l}\text { SEER } \\
2010-2013\end{array}$} \\
\hline & No. & $(\%)$ & No. & $(\%)$ & No. & $(\%)$ & No. & $(\%)$ \\
\hline \multicolumn{9}{|l|}{ Receptor groups } \\
\hline Her2+/HR+ & 562 & $(12.0)$ & 2,616 & $(11.2)$ & & & & \\
\hline Her2+/HR- & 253 & $(5.4)$ & 1,137 & $(4.9)$ & & & & \\
\hline Her2-/HR+ & 3,180 & $(67.8)$ & 15,528 & $(66.6)$ & & & & \\
\hline Triple Negative & 544 & $(11.6)$ & 2,481 & $(10.6)$ & & & & \\
\hline Unknown & 152 & $(3.2)$ & 1,540 & $(6.6)$ & & & & \\
\hline \multicolumn{9}{|l|}{ Estrogen-receptor } \\
\hline Positive & 3,702 & (78.9) & 12,926 & (79.9) & & & & \\
\hline $\begin{array}{l}\text { Negative/ } \\
\text { Borderline }\end{array}$ & 841 & $(17.9)$ & 2,875 & $(17.8)$ & & & & \\
\hline Unknown & 148 & $(3.2)$ & 371 & $(2.3)$ & & & & \\
\hline \multicolumn{9}{|l|}{$\begin{array}{l}\text { Progesterone- } \\
\text { receptor }\end{array}$} \\
\hline Positive & 3,049 & $(65.0)$ & 11,317 & $(70.0)$ & & & & \\
\hline $\begin{array}{l}\text { Negative/ } \\
\text { Borderline }\end{array}$ & 1,490 & $(31.8)$ & 4,442 & $(27.4)$ & & & & \\
\hline Unknown & 152 & $(3.2)$ & 413 & $(2.6)$ & & & & \\
\hline
\end{tabular}

Missing data in the NBCA dataset

${ }^{1}$ Missing data invasive breast cancer patients $n=50$

${ }^{2}$ Missing data DCIS patients $n=28$

${ }^{3}$ Missing data invasive breast cancer patients $n=1630$

${ }^{4}$ Missing data DCIS patients $n=524$

\section{DCIS patients: mastectomy and IBR rates}

During the study period, a total of 9,886 women were diagnosed with DCIS in the NBCA cohort of which $30.8 \%(n=3,048)$ patients underwent mastectomy. The SEER database comprised a total of 60,683 DCIS patients. Thirty-one percent $(n=19,179)$ underwent mastectomy. In the NBCA cohort, mastectomy rates decreased slightly over time (from $32.3 \%$ to $30.7 \%, p<0.001$ ) (Figure 3a). Within the SEER cohort, mastectomy rates initially decreased from 32.0\% in 2010 to 30.2\% in 2011, followed by an increase to 31.9\% in 2013, $(p<0.08)$ (Figure 3a). Of women undergoing mastectomy, $44.0 \%$ of the NBCA cohort and $36.3 \%$ of the SEER cohort underwent IBR. Implant-based breast reconstruction was performed in $87.5 \%$ of the NBCA cohort versus $46.3 \%$ of the SEER cohort. Rates of immediate breast reconstruction increased significantly in both cohorts (from $41.2 \%$ to $49.7 \%$ in the NBCA $(p<0.001)$ and from $34.8 \%$ to $38.7 \%$ in the SEER cohort $(p<0.001)$ (Figure 3b). Table 1 demonstrates the overall characteristics. The majority of patients in the NBCA dataset were aged 50 - 59 years (46.0\%), and the majority of patients in the SEER dataset were aged $40-49$ years old (36.2\%). Tumor localization was nearly similar in both the NBCA and SEER database ( $51.4 \%$ vs $50.6 \%$ left sided and $48.6 \%$ vs $49.4 \%$ right sided, respectively). 


\section{Mastectomy in DCIS patients}

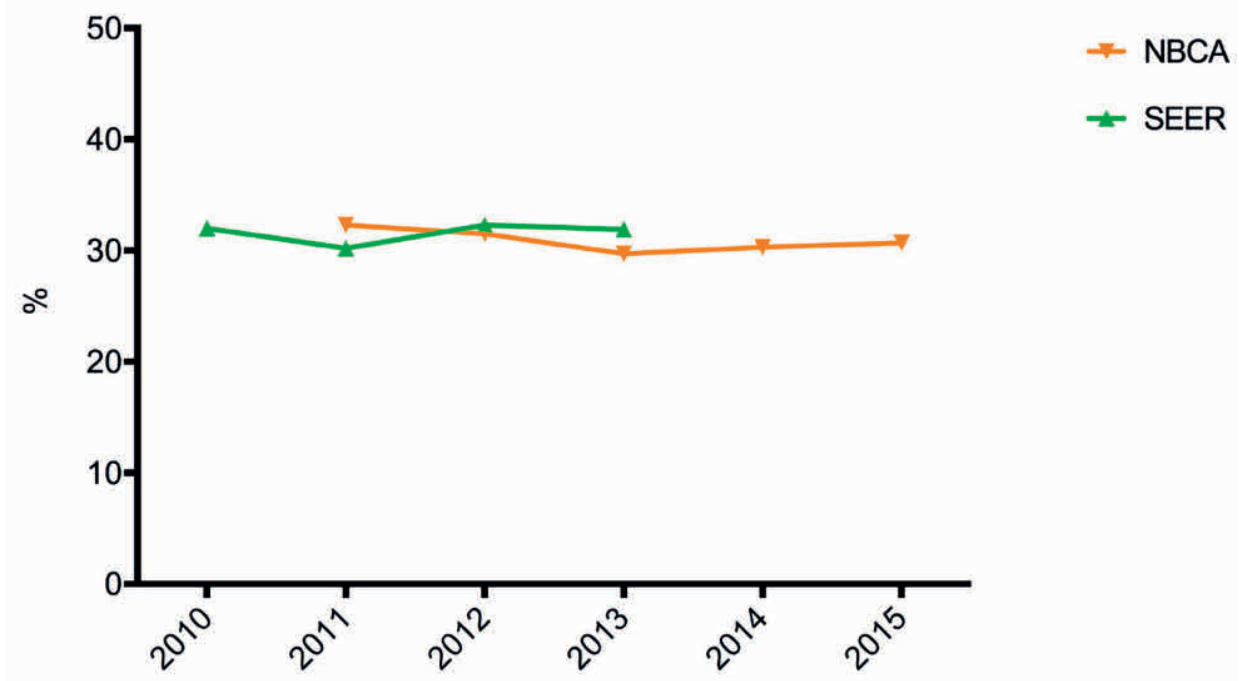

Figure 3a. Trend over time mastectomy in ductal carcinoma in situ patients. Trend over time $p<0.001$

\section{Post-mastectomy immediate breast reconstruction in DCIS patients}

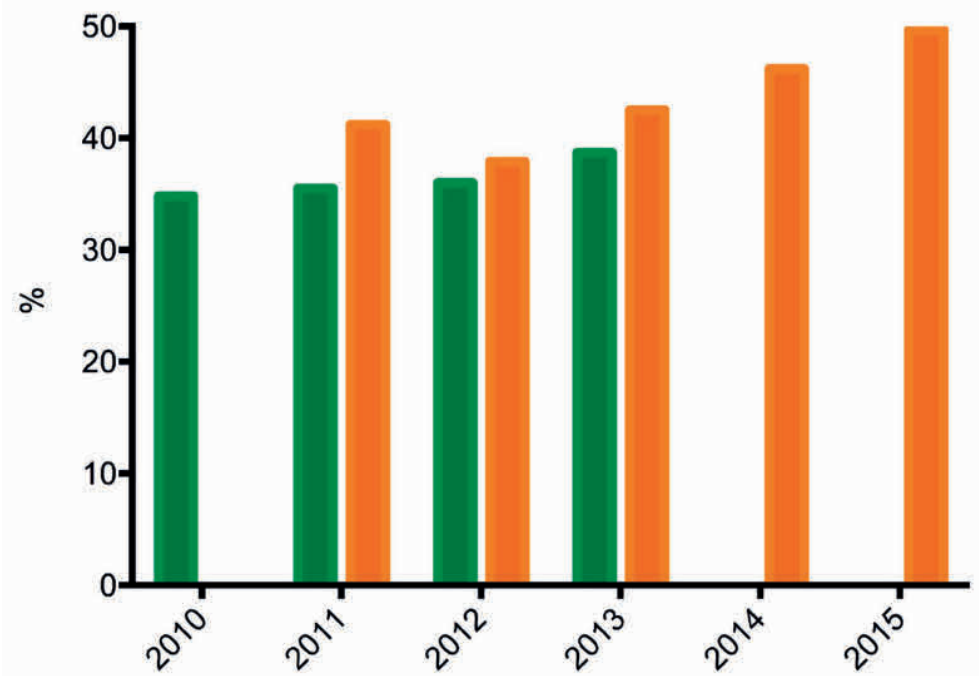

Figure 3b. Trend over time immediate breast reconstruction in ductal carcinoma in situ patients. Trend over time $p<0.001$ 


\section{DISCUSSION}

With breast cancer surgery, large-volume national databases are being increasingly utilized to study outcomes. ${ }^{1-3,6,9,22,55-58}$ Derivations of data from large, heterogeneous populations result in a better overview of surgical intervention, immediate breast reconstructive procedures, but also result in assessment of quality improvement measures and treatment protocols. These large-volume national datasets have the ability to compare and contrast treatment patterns internationally and to further improve breast cancer care. We conducted a proof of concept study exploring the feasibility of combining two large existing operational databases in two countries. This study shows that despite a variety in data items and definitions, some international data can be pooled in order to compare variation in practices and countries. To our knowledge, no comparison of international data registries in the field of IBR has been made to date.

While randomized controlled trials (RCT) remain the gold standard for answering clinical questions, RCTs are often not feasible. ${ }^{59}$ Observational cohort studies, therefore have come to be the standard for clinical research in many surgical fields. As data collection is done in collaboration with the cancer registry using the existing infrastructures, trained objective data-managers using international coding rules, these studies can be completed at low costs. With the number of countries currently developing and conducting research with national registries, it is of utmost importance for the international community to unify and to collaborate in order to improve care and add true value to our patients. ${ }^{60}$ This can be done by international benchmarking and identifying the most successful treatment strategies. ${ }^{61}$

In this study, we demonstrated that it was possible to match and compare $8 \%$ of the available data variables. A major drawback to be able to match variables were the differing data definitions and inclusion criteria. Direct comparisons of health outcomes between countries are rarely done because clinical information contained in administrative or clinical databases cannot be aggregated into a single, searchable database. Although the recording of hospital discharge information may be standardized within countries, it is not so across international borders. Generally speaking, cancer registries have international coding rules, supported by the International Association for Cancer Registries and the European Network for Cancer Registries. Yet differences in diagnostic and treatment coding rules and the lack of standardized health outcomes measurements hinders optimal monitoring and comparison of the quality of health care across different settings globally.

Recent attempts have been made to facilitate international collaboration in order to achieve optimal health outcomes. One example is "The Dr. Foster Global Comparators Project". ${ }^{18}$ By pooling discharge information from 31 healthcare institutions in five countries, this project aimed to allow direct comparisons of healthcare activity. The group found that there were five separate coding systems for entering clinical 
information into administrative data systems. In order to compare outcome information, the authors had to reconcile these coding systems. Although this project is an excellent first step towards attempting to stratify and compare various international datasets, it remains limited to hospital specific data.

To show the value of comparing countries on their treatment patterns IBR rate was studied. Similar to previous studies, we found increasing IBR rates within the SEER cohort, with rates increasing from $21.7 \%$ in 2010 to $26.6 \%$ in 2013 in invasive breast cancer patients and from $34.8 \%$ in 2010 to $38.7 \%$ in 2013 in DCIS patients. ${ }^{9,62}$ Also in the Netherlands, the utilization of IBR increased significantly over time: from $14.4 \%$ in 2011 to $26.0 \%$ in 2015 in invasive breast cancer patients, and from 41.2\% in 2011 to $49.7 \%$ in 2015 in DCIS patients. In line with our results, a recent report by the Dutch Institute for Clinical Auditing (DICA) revealed that on average, 17\% of invasive breast cancer patients and $42 \%$ of DCIS patients undergo IBR. ${ }^{52,63}$

By matching the variables, we were able to provide an insight into the different types of breast reconstruction used in the United States and the Netherlands. Interestingly, the type of immediate reconstruction performed differed between the two countries. The findings of this study are in line with previously published data of type of reconstruction chosen in both countries. ${ }^{39,41,50,63}$ Even though we were only able to match nine variables, we were able to demonstrate the significant differences in treatment modalities. One reason for the low matching rate might be the provision of variables that are not related to breast cancer in the SEER database.

With this intercontinental collaboration and comparison of national data, we have taken the first step towards analyzing comparability of and availability of items in different databases. In general, we strongly endorse international defining items and sharing of national country level registries in order to enhance patient care; harmonization of the databases utilized to study these topics is of utmost importance in order to correctly interpret the data. Therefore, we urge the plastic surgical and breast surgery community to enhance the unification of defining a necessary item set (minimal dataset) on treatment for national databases to compare and contrast treatment patterns internationally in order to further improve breast cancer care.

International registries as instruments of knowledge are extremely useful in offering large amounts of data for performing analyses and deriving information. Evidence derived from large registries heavily influences clinical practice and management strategies. The vast majority of research utilized in treatment guidelines around the globe, and also in the Netherlands, stem from the United States. ${ }^{64}$ Inherently, these reports reflect the viewpoint of the country of origin. To our knowledge, this study is a first of its kind in breast cancer surgery research comparing the similarities and the differences between countries across the Atlantic. We hope that this will be a first step in initiating collaborative national large database studies from different parts of the world. In addition, the authors aimed to initiate conversation regarding unification of databases and data variables. Furthermore, due to the ever-increasing developments in 
the field of reconstructive surgery, and moreover in this era of globalization, proper and complete information on current international trends and different practices around the globe is of utmost importance. Moreover, this paper provides a general overview of IBR rates between the United States and the Netherlands.

This study is not exempt from limitations. One of the authors' goals was to understand the incidence of breast reconstruction both in the United States and in the Netherlands. In addition, it is important to realize that patient reported outcomes and more granular patient data is at times lacking within these large national databases. It is our hope that future database collection processes will be able to include more granular information. All data presented are collected from national databases, which are subject to coding errors, and poor or no long-term follow-up. Moreover, the NBCA data is an extraction of the NCR. The NCR obtains data on follow-up (actively gathered) and vital status (linkage to municipality registries) on a national basis, but does not transfer this to the NBCA database, because these data items are not used to generate an indicator for quality of care. Therefore, the aim of a registration should be taken into account by the user and linkage to more complete sources such as the NCR should be considered. Second, due to the nature of the databases, clinical outcome data was not captured and therefore not analyzed. Furthermore, the collected data entailed different time periods.

\section{CONCLUSION}

The aim of this proof of concept study was to demonstrate that it was possible to compare national registries. This study provides a first overview of merging data from different datasets and as an example compare treatment patterns in IBR over time across to nations; the Netherlands and the United States of America. This study reveals the usability and value of comparable datasets. We advocate for international databases to align their variables in order to improve quality of breast cancer care for all breast cancer patients across the globe. 


\section{TABLE AND FIGURE LEGEND}

Table 1. Overall characteristics invasive breast cancer and ductal carcinoma in situ patients

Figure 1. Overview matched variables NBCA and SEER

Figure 2a. Trend over time mastectomy in invasive breast cancer patients Trend over time $p<0.001$

Figure $2 \mathrm{~b}$. Trend over time immediate breast reconstruction in invasive breast cancer patients

Trend over time $p<0.001$

Figure 3a. Trend over time mastectomy in ductal carcinoma in situ patients Trend over time $p<0.001$

Figure $3 \mathrm{~b}$. Trend over time immediate breast reconstruction in ductal carcinoma in situ patients

Trend over time $p<0.001$ 


\section{REFERENCES}

1. Albornoz CR, Bach PB, Mehrara BJ, et al. A paradigm shift in U.S. Breast reconstruction: increasing implant rates. Plastic and reconstructive surgery. 2013;131(1):15-23.

2. Cemal Y, Albornoz CR, Disa JJ, et al. A paradigm shift in U.S. breast reconstruction: Part 2. The influence of changing mastectomy patterns on reconstructive rate and method. Plastic and reconstructive surgery. 2013;131(3):320e-326e.

3. Habermann EB, Thomsen KM, Hieken TJ, Boughey JC. Impact of availability of immediate breast reconstruction on bilateral mastectomy rates for breast cancer across the United States: data from the nationwide inpatient sample. Annals of surgical oncology. 2014;21(10):3290-3296.

4. Yang RL, Newman AS, Lin IC, et al. Trends in immediate breast reconstruction across insurance groups after enactment of breast cancer legislation. Cancer. 2013;119(13):2462-2468.

5. Butler PD, Nelson JA, Fischer JP, et al. Racial and age disparities persist in immediate breast reconstruction: an updated analysis of 48,564 patients from the 2005 to 2011 American College of Surgeons National Surgery Quality Improvement Program data sets. American journal of surgery. 2015.

6. Silva AK, Lapin B, Yao KA, Song DH, Sisco M. The Effect of Contralateral Prophylactic Mastectomy on Perioperative Complications in Women Undergoing Immediate Breast Reconstruction: A NSQIP Analysis. Annals of surgical oncology. 2015;22(11):3474-3480.

7. Alderman AK, Hawley ST, Janz NK, et al. Racial and ethnic disparities in the use of postmastectomy breast reconstruction: results from a population- based study. Journal of clinical oncology : official journal of the American Society of Clinical Oncology. 2009;27(32):5325-5330.

8. Alderman AK, Wei Y, Birkmeyer JD. Use of breast reconstruction after mastectomy following the Women's Health and Cancer Rights Act. Jama. 2006;295(4):387-388.

9. Lang JE, Summers DE, Cui H, et al. Trends in post-mastectomy reconstruction: a SEER database analysis. Journal of surgical oncology. 2013;108(3):163-168.

10. Ravdin PM, Cronin KA, Howlader N, et al. The decrease in breast-cancer incidence in 2003 in the United States. The New England journal of medicine. 2007;356(16):1670-1674.

11. Ong WL, Schouwenburg MG, van Bommel AC, et al. A Standard Set of Value-Based Patient-Centered Outcomes for Breast Cancer: The International Consortium for Health Outcomes Measurement (ICHOM) Initiative. JAMA Oncol. 2016.

12. Cooter RD, Barker S, Carroll SM, et al. International importance of robust breast device registries. Plastic and reconstructive surgery. 2015;135(2):330-336.

13. Myhr KM, Grytten N, Torkildsen O, et al. A need for national registries and international collaborative research in multiple sclerosis. Acta neurologica Scandinavica Supplementum. 2012(195):1-3.

14. Fortier I, Doiron D, Little J, et al. Is rigorous retrospective harmonization possible? Application of the DataSHaPER approach across 53 large studies. International journal of epidemiology. 2011;40(5):13141328.

15. Rolland B, Reid S, Stelling D, et al. Toward Rigorous Data Harmonization in Cancer Epidemiology Research: One Approach. American journal of epidemiology. 2015;182(12):1033-1038.

16. de Leede EM, Sibinga Mulder BG, Bastiaannet E, et al. Common variables in European pancreatic cancer registries: The introduction of the EURECCA pancreatic cancer project. European journal of surgical oncology : the journal of the European Society of Surgical Oncology and the British Association of Surgical Oncology. 2016;42(9):1414-1419.

17. Breugom AJ, Bastiaannet E, Boelens PG, et al. Adjuvant chemotherapy and relative survival of patients with stage II colon cancer - A EURECCA international comparison between the Netherlands, Denmark, Sweden, England, Ireland, Belgium, and Lithuania. European journal of cancer. 2016;63:110-117.

18. Bottle A, Middleton S, Kalkman CJ, Livingston EH, Aylin P. Global comparators project: international comparison of hospital outcomes using administrative data. Health services research. 2013;48(6 Pt 1):2081-2100. 
19. Kiderlen M, Walsh PM, Bastiaannet E, et al. Treatment strategies and survival of older breast cancer patients - an international comparison between the Netherlands and Ireland. PloS one. 2015;10(2):e0118074.

20. Davies J, Yudkin JS, Atun R. Liberating data: the crucial weapon in the fight against NCDs. The lancet Diabetes \& endocrinology. 2016;4(3):197-198.

21. Garcia-Etienne CA, Tomatis M, Heil J, et al. Mastectomy trends for early-stage breast cancer: a report from the EUSOMA multi-institutional European database. European journal of cancer. 2012;48(13):19471956.

22. Kamali P, Koolen PG, Ibrahim AM, et al. Analyzing Regional Differences over a 15-Year Trend of OneStage versus Two-Stage Breast Reconstruction in 941,191 Postmastectomy Patients. Plastic and reconstructive surgery. 2016;138(1):1e-14e.

23. Kummerow KL, Du L, Penson DF, Shyr Y, Hooks MA. Nationwide trends in mastectomy for early-stage breast cancer. JAMA surgery. 2015;150(1):9-16.

24. Neuburger J, Macneill F, Jeevan R, van der Meulen JH, Cromwell DA. Trends in the use of bilateral mastectomy in England from 2002 to 2011: retrospective analysis of hospital episode statistics. BMJ open. 2013;3(8).

25. Pesce CE, Liederbach E, Czechura T, Winchester DJ, Yao K. Changing surgical trends in young patients with early stage breast cancer, 2003 to 2010: a report from the National Cancer Data Base. J Am Coll Surg. 2014;219(1):19-28.

26. Roth RS, Lowery JC, Davis J, Wilkins EG. Quality of life and affective distress in women seeking immediate versus delayed breast reconstruction after mastectomy for breast cancer. Plastic and reconstructive surgery. 2005;116(4):993-1002; discussion 1003-1005.

27. Girotto JA, Schreiber J, Nahabedian MY. Breast reconstruction in the elderly: preserving excellent quality of life. Annals of plastic surgery. 2003;50(6):572-578.

28. Maly RC, Liu Y, Kwong E, Thind A, Diamant AL. Breast reconstructive surgery in medically underserved women with breast cancer: the role of patient-physician communication. Cancer. 2009;115(20):48194827.

29. Anderson SG, Rodin J, Ariyan S. Treatment considerations in postmastectomy reconstruction: their relative importance and relationship to patient satisfaction. Annals of plastic surgery. 1994;33(3):263270; discussion 270-261.

30. Rowland JH, Desmond KA, Meyerowitz BE, Belin TR, Wyatt GE, Ganz PA. Role of breast reconstructive surgery in physical and emotional outcomes among breast cancer survivors. Journal of the National Cancer Institute. 2000;92(17):1422-1429.

31. Schain WS. Breast reconstruction. Update of psychosocial and pragmatic concerns. Cancer. 1991;68(5 Suppl):1170-1175.

32. Lee C, Sunu C, Pignone M. Patient-reported outcomes of breast reconstruction after mastectomy: a systematic review. J Am Coll Surg. 2009;209(1):123-133.

33. Wong A, Snook K, Brennan M, et al. Increasing breast reconstruction rates by offering more women a choice. ANZ journal of surgery. 2014;84(1-2):31-36.

34. Dutch Breast Cancer Guideline. https://richtlijnendatabase.nl/en/richtlijn/breast_cancer/breast_cancer. html. Assessed: December 16th, 2016

35. Early and locally advanced breast cancer: diagnosis and treatment. NICE guideline. February 2009. National Collaborating Centre for Cancer.

36. Jagsi R, Jiang J, Momoh AO, et al. Trends and variation in use of breast reconstruction in patients with breast cancer undergoing mastectomy in the United States. Journal of clinical oncology : official journal of the American Society of Clinical Oncology. 2014;32(9):919-926.

37. Habermann EB, Abbott A, Parsons HM, Virnig BA, Al-Refaie WB, Tuttle TM. Are mastectomy rates really increasing in the United States? Journal of clinical oncology : official journal of the American Society of Clinical Oncology. 2010;28(21):3437-3441.

38. Tuttle TM, Rueth NM, Abbott A, Virnig BA. Trends in the local treatment of breast cancer: should we be worried? Journal of surgical oncology. 2011;103(4):313-316. 
39. Wilkins EG, Alderman AK. Breast reconstruction practices in north america: current trends and future priorities. Seminars in plastic surgery. 2004;18(2):149-155.

40. Agarwal S, Pappas L, Neumayer L, Agarwal J. An analysis of immediate postmastectomy breast reconstruction frequency using the surveillance, epidemiology, and end results database. The breast journal. 2011;17(4):352-358.

41. Alderman AK, McMahon L, Jr., Wilkins EG. The national utilization of immediate and early delayed breast reconstruction and the effect of sociodemographic factors. Plastic and reconstructive surgery. 2003;111(2):695-703; discussion 704-695.

42. Macadam SA, Ho AL, Lennox PA, Pusic AL. Patient-reported satisfaction and health-related quality of life following breast reconstruction: a comparison of shaped cohesive gel and round cohesive gel implant recipients. Plastic and reconstructive surgery. 2013;131(3):431-441.

43. Atisha D, Alderman AK, Lowery JC, Kuhn LE, Davis J, Wilkins EG. Prospective analysis of long-term psychosocial outcomes in breast reconstruction: two-year postoperative results from the Michigan Breast Reconstruction Outcomes Study. Annals of surgery. 2008;247(6):1019-1028.

44. Kamali P, Paul MA, Ibrahim AM, et al. National and Regional Differences in 32,248 Postmastectomy Autologous Breast Reconstruction Using the Updated National Inpatient Survey. Annals of plastic surgery. 2017.

45. Kamali P, Koolen PG, Paul MA, Medin C, Shermerhorn M, Lin SJ. Regional and National Trends Over 20 Years in One-Stage vs Two-staged Implant Based Breast Reconstruction. Plastic and reconstructive surgery. 2015;136(4 Suppl):122.

46. Bucknor A, Chattha A, Ultee K, et al. The financial impact and drivers of hospital charges in contralateral prophylactic mastectomy and reconstruction: a Nationwide Inpatient Sample hospital analysis. Breast cancer research and treatment. 2017.

47. Kamali $\mathrm{P}$, Curiel $\mathrm{D}$, van Veldhuisen $\mathrm{CL}$, et al. Trends in immediate breast reconstruction and early complication rates among older women: A big data analysis. Journal of surgical oncology. 2017;115(7):870-877.

48. Kamali P, Zettervall SL, Wu W, et al. Differences in the Reporting of Racial and Socioeconomic Disparities among Three Large National Databases for Breast Reconstruction. Plastic and reconstructive surgery. 2017;139(4):795-807.

49. Kamali P, Paul MA, Ibrahim AMS, et al. National and Regional Differences in 32,248 Postmastectomy Autologous Breast Reconstruction Using the Updated National Inpatient Survey. Annals of plastic surgery. 2017;78(6):717-722.

50. NABON Breast Cancer Audit (NBCA). Available at: https://www.dica.nl/nbca/home. Assessed: June 1st 2016.

51. van Bommel AC, Spronk PE, Vrancken Peeters MT, et al. Clinical auditing as an instrument for quality improvement in breast cancer care in the Netherlands: The national NABON Breast Cancer Audit. Journal of surgical oncology. 2017;115(3):243-249.

52. van Bommel AC, Mureau MA, Schreuder K, et al. Large variation between hospitals in immediate breast reconstruction rates after mastectomy for breast cancer in the Netherlands. J Plast Reconstr Aesthet Surg. 2017;70(2):215-221.

53. National Cancer Institute, Surveillance Epidemiology, and End Result (SEER) data description. Available at: http://seer.cancer.gov/data/ Assessed: 1 Dec. 2015.

54. SEER Data Elements Description. Available at: https://seer.cancer.gov/data/seerstat/nov2015/TextData. FileDescription.pdf. Assessed: February 23rd 2017.

55. Bleicher RJ, Ruth K, Sigurdson ER, et al. Time to Surgery and Breast Cancer Survival in the United States. JAMA Oncol. 2016;2(3):330-339.

56. Frasier LL, Holden S, Holden T, et al. Temporal Trends in Postmastectomy Radiation Therapy and Breast Reconstruction Associated With Changes in National Comprehensive Cancer Network Guidelines. JAMA Oncol. 2016;2(1):95-101.

57. Fischer JP, Wes AM, Tuggle CT, et al. Mastectomy with or without immediate implant reconstruction has similar 30-day perioperative outcomes. J Plast Reconstr Aesthet Surg. 2014;67(11):1515-1522. 
58. Nwaogu I, Yan Y, Margenthaler JA, Myckatyn TM. Venous Thromboembolism after Breast Reconstruction in Patients Undergoing Breast Surgery: An American College of Surgeons NSQIP Analysis. J Am Coll Surg. 2015;220(5):886-893.

59. Tannock IF, Amir E, Booth CM, et al. Relevance of randomised controlled trials in oncology. The Lancet Oncology. 2016;17(12):e560-e567.

60. Porter ME. What is value in health care? The New England journal of medicine. 2010;363(26):2477-2481.

61. James S, Rao SV, Granger CB. Registry-based randomized clinical trials--a new clinical trial paradigm. Nature reviews Cardiology. 2015;12(5):312-316.

62. Agarwal S, Kidwell KM, Farberg A, Kozlow JH, Chung KC, Momoh AO. Immediate Reconstruction of the Radiated Breast: Recent Trends Contrary to Traditional Standards. Annals of surgical oncology. 2015;22(8):2551-2559.

63. Annual report Dutch Institute for Clinical Auditing 2013. Available at: https://www.dica.nl/media/332/ DICA-jaarrapportage-2015.pdf Assessed: 1 April 2016.

64. Dutch Breast Reconstruction Guideline. https://richtlijnendatabase.nl/en/richtlijn/breast_reconstruction/ breast_reconstruction_after_mastectomy.html Assessed: September 152016. 


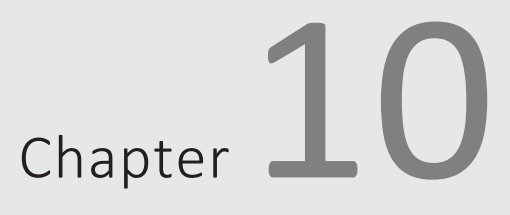

Discussion and future perspectives 

The preceding work illustrates the utility of "big data" in the form of large-volume databases to study trends and variation in patients undergoing breast reconstructive surgery. But what are the limitations and drawbacks of big data and large-volume databases? What is the current state of big data in healthcare globally? What about future perspectives? Moreover, how will the future of plastic surgery be impacted and what is the role of national and international plastic surgery societies? In this chapter, we will elaborate on all of the above questions, starting with the limitations of the largevolume databases used in this thesis. We then take a step back and discuss big data in a global context, touching on the current state, challenges and ongoing efforts to address these challenges. We continue the discussion by elaborating on the role of artificial intelligence, where we are now and what the future might look like in the field plastic surgery. We conclude by discussing the role of national plastic surgery societies in the era of big data.

\section{LIMITATIONS OF LARGE-VOLUME DATABASES}

National registries can be designed to capture entire population data, acts as a surveillance tool, or monitor longitudinal trends. A robust registry can provide true population estimates and can detect small differences with remarkable statistical power. The success of a registry relies on multiple participating centers and the collection of clinically relevant data. Technological advancements, such as HIT and EHR have unlocked new possibilities for efficient data capture and widespread opportunities to merge massive datasets, particularly in the setting of national registries and administrative data. These large-volume databases provide a unique opportunity for observational plastic surgical research to examine research questions that cannot be examined in most RCTs. This being said, big data and large-volume databases do not only have immense potential, but also several limitations. Although large-volume databases play an important role in plastic surgery outcomes research, results from these studies should be viewed critically. Large-volume administrative database studies have broad generalizability, a large number of patient records, less attrition than clinical trials, are faster and less costly than primary data collection. The limitations of database studies are therefore inherently related to observational study designs and datasets.

\section{Variables}

Similar to a retrospective chart review, researchers are restricted to the data that were collected and recorded in the database. For example, administrative databases, such as the NIS, lack clinically relevant data, such as cancer stage and histology, family history of breast cancer, surgical procedure specific information and other information that can influence the decision-making process for the type of mastectomy and reconstruction 
chosen within the plastic surgery population. Clinical databases, such as the ACS-NSQIP, SEER and NBCA are developed with specific clinical goals in mind and therefore populated with defined patient information but certain data points of interest may be missing. As we demonstrated in chapter 5, in the ACS-NSQIP database, complications are limited to 30 days postoperatively. Complications occurring after 30 days are not captured. Although this 30-day window may be sufficient to capture most medical complications, in patient undergoing plastic surgical operation it sometimes takes longer than 30 days for complications, such as fat necrosis or abdominal wall hernia to become evident.

\section{Coding}

A common limitation shared by the majority of administrative databases is that they are dependent on ICD-9 and/or CPT codes to isolate comorbidities, diagnoses, procedures and complications. These codes were originally not developed for research purposes and their use may only be valid for certain diagnoses, procedures or complications. Details that may be lacking include disease severity, tumor size, lymph node status and tumor grading. Second, the extraction of these CPT and ICD-9 codes often comes from insurance claims or hospital-level records which may be influenced by reimbursement strategies or coded by non-medical team members. Two recent studies demonstrated higher rates of postoperative complications in the NIS database when compared to the ACS-NSQIP database for the same procedure. This despite NIS only capturing inpatient complications while ACS-NSQIP captures morbidity and mortality for up to 30 days after the index procedure. ${ }^{1,2}$ The NIS database uses billing records as a component of its data extraction while the ACS-NSQIP data is collected by a trained reviewer using established definitions. And finally, variations in coding may be due to clerical errors or various interpretations of discharge summaries, operative records, or other healthcare documentation. Variation in coding can also be due to annual updates of ICD-9 and CPT codes, in which the definition of a particular code may change. For example, in 2009 the ICD-9 code for deep venous thrombosis changed to differentiate acute from chronic deep venous thrombosis events. The international community has made significant steps to address these problems by introducing ICD-10 codes. The overall number of codes and diagnoses have significantly increased when going from ICD-9 (+/- 17,000

codes) to ICD-10 $(>155,000)$ codes. $^{3}$ However, the transition period might not produce reliable data.

\section{Sample size selection}

Because large-volume database studies are not randomized, sample selection bias is the greatest design limitation, whereby observed outcomes may differ because patients differed at baseline on factors other than treatment. Important baseline variables known to impact outcomes must be available and reliable, and variables can sometimes 
be combined to obtain information that is not available from a single variable. Sampling of the type of hospital is another factor that may influence the sample selection. As demonstrated in chapter 7, the ACS-NSQIP, NIS, and the SEER databases are used extensively as a nationwide representation of the U.S. population, but major variations exist in the states covered by the different databases. For example, the NIS and the ACS-NSQIP reflect practice patterns in 47 or more states, whereas the SEER database reflects practice in only 17 states. Moreover, the SEER database lacks coverage of the majority of the major cancer centers throughout the United States.

\section{Other limitations}

Other limitations include the inability to determine surgical or treatment quality, lack of functional and aesthetic outcomes, and the potential inability to follow patients over time. The large number of patient encounters or procedures found in these databases often leads to statistical significance where real clinical significance may be lacking.

\section{BIG DATA IN HEALTHCARE: A GLOBAL VIEW}

Over the last years, the international community has increasingly recognized the importance of health information technology and the potential impact of big data in the healthcare setting. Globally, investment in healthcare big data was estimated to be $\$ 14.25$ billion in 2017 . This investment is predicted to increase to over $\$ 68.75$ billion by the end of $2025 .{ }^{4}$ In terms of return of investment, estimates predict that implementation of nationwide health information technology will result in a reduction of 12 to 17 percent of the $\$ 2.6$ trillion baseline US healthcare costs. This equates to up to $\$ 300$ to $\$ 450$ billion in savings by 2020 in the United States alone. ${ }^{5,6}$ Similar potential savings in healthcare costs have been estimated for Europe., 6 Moreover, the European Union has recently announced a \$24 billion investment in artificial intelligence, aiming to transform the field of big data, including healthcare big data. ${ }^{8}$ Currently, Asia and the United States each invest more in Al than three times that of Europe.

Despite increasing awareness of the benefits of big data and the related methodological and technological advances that are being made, many countries appear to be slow in adopting approaches based on such data. ${ }^{9}$ The reasons may include gaps in funding, leadership and technical expertise, and competing priorities within the health system. ${ }^{10}$ Many governments are still considering appropriate policy options. In 2015, according to the World Health Organization's Global Observatory for eHealth, only 21 (17\%) of the 125 Member States surveyed reported having a policy or strategy regulating the use of big data in their health sectors. ${ }^{11}$

One of the most promising examples of big data in global health may emerge from India's ambitious personal identification program. Since 2010 the government of India 
has been issuing Aadhaar cards and unique identifying numbers to all 1.2 billion of its citizens. ${ }^{12}$ The cards, numbers and associated biometric identification offer the possibility of generating and monitoring health and social data - including electronic medical records and information on health insurance for low-income families - on a huge scale. ${ }^{12,13}$ Even if limited in its current reach, the Aadhaar system forms a backbone that could allow the more reliable and extensive collection of health statistics. This, in turn, could lead to dramatic improvements in the planning and delivery of public health interventions.

\section{Medical Record: Toward Universal Electronic Health Records?}

In the fifth century B.C., medical reporting was highly influenced by Hippocrates. He advocated that the medical record serve two goals: it should accurately reflect the course of disease, and it should indicate the possible causes of disease. ${ }^{14}$ Patient records date back to ancient civilization. Traditionally, the record was for the benefit of the physician; in its purest form, the record told the medical story of a patient, enabling the doctor to remember details that would otherwise be lost to memory. As technology continued to improve and develop, medical records took on entirely new functions in the latter part of the 20th century. More recently, paper records are increasingly being replaced with electronic health records (EHRs). If used and shared effectively, EHRs have the potential to improve the quality and convenience of health care and the effectiveness of health research.

In 2009, the United States Congress passed The Health Information Technology for Economic and Clinical Health (HITECH) act, aiming to give every American access to private, secure, and portable electronic health records. ${ }^{15}$ Under the HITECH Act, the United States Department of Health and Human Services invested \$25.9 billion to promote and expand the adoption of health information technology. As part of this bill, tens of billions of dollars were invested in incentives for healthcare providers to implement EHRs. In 2008, prior the enactment of HITECH, 17 percent of physicians were using advanced electronic health records and just 9 percent of hospitals had adopted electronic health records throughout the United States. ${ }^{16}$ Recent studies reveal a significant increase in the adoption of EHRs since the implementation of the HITECH; over 87 percent of physicians and over 95 percent of hospitals have adopted any type of EHR. ${ }^{17,18}$ While the United States has made substantial progress in digitizing health records, most patients are still unable to easily transfer their complete medical records to a new provider, share their records securely with other family members or caregivers, or donate their medical data for research.

In Europe, The European Commission has set a goal to enable all Europeans to have access to online medical records anywhere in Europe by $2020 .{ }^{19}$ Following the enactment of Article 14 of Directive 2011/24/EU on patients' rights in cross-border healthcare, the eHealth Network was set up to facilitate the cooperation between the 
European eHealth systems and to draw up a series of guidelines to facilitate the crossborder transferability of medical data, taking into account the EU data protection rules. $^{20}$ That being said, there are broad differences within and across Europe with regards to privacy protection and data sharing policies. To address this problem, since 2014, the EU has implemented a European Health Parliament ('EHP') to explore solutions to Europe's healthcare problems. ${ }^{21}$ The topic of 'big data in healthcare' has been discussed, with one EHP proposal being the creation of a Europe-wide connected Electronic Health Records Organization ('EHRO'), with the intention being to collect and use patients' health data across member states and thus empower patients and enable better health outcomes. Although significant steps have been made toward this goal, the adoption of EHRs within Europe varies greatly. ${ }^{22}$

\section{How Safe is Your Data?}

The concept of a centralized supranational central server raises concerns about storing electronic medical records in a single location. The privacy threat posed by a supranational network is a key concern. Cross-border and interoperable electronic health record systems make confidential data more easily and rapidly accessible to a wider audience and increase the risk that personal data concerning health could be accidentally exposed or easily distributed to unauthorized parties by enabling greater access to a compilation of the personal data concerning health, from different sources, and throughout a lifetime. In 2015, one major US health insurer reported that hackers had broken into its database and stolen records that contained personal information for approximately 80 million customers and employees. ${ }^{23}$ The following year, Community Health Systems fell victim to the computer bug Heartbleed and data from 5.4 million patients was taken. ${ }^{24}$ More recently, in 2017 a cyberattack on the NHS in the UK paralyzed the entire computer system. Patients were told to stay home; doctors and nurses were unable to access email or medical records and had to take notes by hand. The hackers demanded a ransom, to be paid in bitcoin. ${ }^{25}$ Medical data is a popular target for criminals. Reuters estimates that medical information is worth 10 times more than credit card information on the black market as perpetrators attempt to receive treatment they would otherwise pay for. ${ }^{26}$ At the national and international level, effective strategies for data integration, interoperability and security standards are essential to assure data safety and security.

\section{Ownership of Data}

As discussed above, patient medical records are undergoing a seismic shift. The transition from paper to electronic records has created new opportunities for sharing information among healthcare providers, between physicians and patients, and with third parties. However, the question remains; who owns this data? In general, 
ownership of information belongs to the individual or company who created or authored that information. For example, intellectual property laws protect "original works of authorship." Medical records represent professional medical opinions of a physician or a medical institution, and therefore may not necessarily be the patient's property. In the United States, every state has different laws about who owns medical record data. ${ }^{27}$ Under federal and state law, patients have legal privacy, security and accuracy rights related to their health information. However, once that information is captured and documented in written or electronic form (e.g., paper chart or electronic data file), and since the health care provider owns the media in which the information is recorded and stored, the health care provider gains the property right of possession of data. In essence, the health care provider becomes the legal custodian of your health care record and is given specific legal rights and duties relating to possession and protection of that health record. Steps have been taken to implement a "patient-driven economy". ${ }^{28}$ In such a scenario, the patient owns his or her data, and can for example sell his or her medical information to a drug company on his or her terms for a specific purpose like drug trail. A recent start-up, Hu-manity.co aims to make this a reality by creating a health data marketplace with help from blockchain. ${ }^{29}$ The start-up has developed proprietary technology that identifies existing legal corridors in privacy regulations, and has designed new intelligent contracts on blockchains which patients can use to negotiate new terms of consent and authorization with corporations. Starting with healthcare data, Hu-manity.co's global inherent Human Data Consent and Authorization Blockchain (HD-CAB) will empower individuals to manage consent and authorization for companies currently using their inherent human datasets. Empowering patients to take control of their data could be of particular importance for cross-border healthcare and health research activities in Europe where healthcare, and the concept of "data ownership" is highly fragmented and multinational. Transfer of medical data from one country to another in the EU is very difficult. Ownership of data by patients could overcome these obstacles and unleash new ways to stimulate a competitive health-driven economy.

\section{Database Alignment and Data Sharing}

Patient registries have for decades served as a key tool for assessing clinical outcomes, and clinical and health technology performance. They provide significant potential for research and public health improvements globally, owing to the large volume of patients in each registry and the variety of quality medical information related to each patient. As a result, hundreds, if not thousands of registries have been set up. These different types of "big data" collectively offer unique opportunities to study patterns of care, guideline adherence, variation in practice, and outcomes following interventions. Comparing these data across populations and time points can facilitate new scientific discoveries, faster access to treatment interventions, and enable differences between 
populations to be delineated, allowing for identification of optimal treatment protocol and strategies aiming to improve quality of care globally. Yet to date, the ability to compare data is greatly limited by the heterogeneity of data elements, variable definitions, inclusion criteria, and coding systems in the registries. Moreover, patient records can be computationally opaque, for example, in the form of free text, recorded speech, or medical images, translation into a format compatible with computational analyses will be necessary. As seen in chapter 8 , data in different languages, timeconsuming searches, and identification are other important barriers. For this reason, direct comparisons of data variables between countries are rarely done because disease and treatment information contained within these datasets cannot be aggregated into a single, searchable database. Although the recording of hospital discharge information may be standardized within countries, it is not the case across international borders. Generally speaking, cancer registries have international coding rules, supported by the International Association for Cancer Registries and the European Network for Cancer Registries. Yet differences in diagnostic and treatment coding rules and the lack of standardized health outcomes measurements hinder optimal monitoring and comparison of the quality of health care across different settings globally.

\section{International Efforts Addressing Current Challenges}

The public health benefits of data sharing have been widely recognized and emphasized over the past decade. ${ }^{30-33}$ The Belmont Report, which provides the framework for human subjects research in the United States, indicates that researchers have a duty to both reduce potential harm to subjects and maximize the possible benefits of the research. ${ }^{34}$ Because sharing data has the potential to positively impact society on the basis of subject participation, by providing greater opportunities for scientific discovery; some argue that researchers may have an ethical duty to share their data unless doing so would increase risk to subjects. ${ }^{35}$ Yet to date, many legal and technical challenges related to the sharing of data exist. As scientific research is progressively dependent on large and complex data sets, they are stored or made available separately from the actual publication because of sheer size. But when data are saved, the ways in which they are stored and catalogued is uneven, making discovery and linking of the data set difficult or even impossible. Over the last decade, the scientific community has increasingly advocated for sharing of data. Other groups have preferred standardization of health outcome measurements. On an international level, different stakeholders have implemented steps toward safely storing, sharing and reusing data across disciplines and borders.

Over the past few years, several organizations have led efforts to address key parts of these problems at a high level, such as the FAIR (findable, accessible, interoperable, and reusable) data principles, the Transparency and openness promotion (TOP) guidelines and the International Consortium for Health Outcomes Measurement (ICHOM). 
Moreover, more recently the European commission has launched the "European Open Science Cloud", a cloud for research data.

The main objective of the FAIR Data Principles is to optimize preparation of research data for man and machine. The FAIR Data Principles are based on four fundamental principles- Findability, Accessibility, Interoperability, and Reusability. The principles support a wide range of new international initiatives, such as the European Open Science Cloud and the NIH big Data to Knowledge. This is done by provision of clear guidelines that ensure all data and associated services in the emergent "Internet of Data" are findable, accessible, interoperable, and reusable, not only by people, but also by machines. Recognizing that computers must be capable of accessing a data publication autonomously, unaided by their human operators, is core to the FAIR Principles. Computers are now an inseparable companion in every research endeavour. Contemporary scientific datasets are large, complex, and globally-distributed, making it almost impossible for humans to manually discover, integrate, inspect, and interpret them. This (re)usability barrier has, until now, prevented us from maximizing the returnon-investment from the massive global financial support of big data research and development projects, especially in the life and health sciences. With respect to Open Science, the FAIR Principles advocate being "intelligently open", rather than "religiously open". The Principles do not propose that all data should be freely available - in particular with respect to privacy-sensitive data. Rather, they propose that all data should be made available for reuse under clearly-defined conditions and licenses, available through a well-defined process, and with proper and complete acknowledgement and citation. Data and metadata should be easy to locate, both by humans and by computer systems. Basic machine-readable descriptive metadata enable the discovery of interesting datasets and services. Each dataset is assigned a globally unique and persistent identifier, (e.g. a Digital Object Identifier (DOI)).

The Transparency and Openness Promotion (TOP) guidelines, founded in 2014, aims to address the journal procedures and policies for publication. ${ }^{36}$ The objectives of the guidelines are "to encourage greater transparency and reproducibility of research in the published record" with the ultimate goal of improving "both research practices and the credibility and reputation of the field of science". ${ }^{37}$ Transparency, openness, and reproducibility are readily recognized as vital features of science. The group states that in the present system, emphasis on innovation may undermine practices that support verification. For example, in purely transparent scientific work, both null results and statistically significant results are made available and help others to accurately assess the evidence base for a phenomenon. In the present culture, however, null results are published less frequently than statistically significant results and are, therefore, more likely to be inaccessible and lost in the "file drawer". ${ }^{38}$ The guidelines promulgate eight transparency standards that can be implemented in whole or part by journals. Journals that adopt the guidelines will choose the level of their implementation for each standard, ranging from level 0 , connoting no implementation of the standard, to level 3 , connoting 
full implementation and active policing of the standard by the journal editors. Adoption of the guidelines will require authors, reviewers, and editors to view research papers through a different lens than they currently use; one that focuses on adherence to the guidelines.

The International Consortium for Health Outcomes Measurement (ICHOM) was founded in 2012 to promote value-based health care by defining global standard sets of outcomes measures and to promote adoption of these measures worldwide. The group aims to transform health care systems globally by measuring and reporting patient outcomes in a standardized fashion. To date, ICHOM has developed 24 standardized sets, one of which addresses breast cancer. ${ }^{39}$ Still this dataset does not describe tumor, diagnostic and treatment data in details, but mainly focusses on patient reported outcomes such as Quality of life.

First launched in 2015, European Open Science Cloud (EOSC) offer Europe's 1.7 million researchers and 70 million science and technology professionals a virtual environment with free, at the point of use, open, and seamless services for storage, management, analysis and re-use of research data, across borders and scientific disciplines. ${ }^{40}$ EOSC will furthermore provide a secure environment where privacy and data protection are guaranteed by design, based on recognized standards, and where users can be confident concerning data security and liability risks. Australia imagines a similarly ambitious future, enabled through its research infrastructure; illustrated most recently in the 2016 National Research Infrastructure Roadmap. ${ }^{41}$ Similar steps towards "cloud computing" in research have been made in other countries. In the United States (US), for example, the National Institutes of Health is promoting the deposition of research data on diverse cloud platforms, including commercial clouds, to facilitate data sharing. ${ }^{42}$

\section{Artificial Intelligence}

In April 2018, there was a public outcry on Facebooks' policy, use and processing of personal data. ${ }^{43}$ It appeared that Facebook had asked several major U.S. hospitals to share anonymized data about their patients, such as illnesses and prescription information for a proposed research project. Facebook was intending to match it up with user data it had collected, and help the hospitals figure out which patients might need special care or treatment. While the data shared would obscure personally identifiable information, such as the patient's name, Facebook proposed using a common computer science technique called "hashing" to match individuals who existed in both sets. This real-life example perfectly reflects the relation and potential use of health data, big data, artificial intelligence and challenges such as privacy.

With the unprecedented growth in healthcare data, organizations are employing analytical tools, artificial intelligence, and machine learning techniques to derive datadriven insights in order to reduce healthcare costs, enhance revenue streams, develop personalized medicine, and manage proactive patient care. Artificial intelligence is the 
concept of machines performing tasks that once required human intelligence to complete. ${ }^{44}$ Examples of the potential use of $\mathrm{Al}$ in healthcare include early detection, diagnosis, decision making, treatment, research and training. ${ }^{45}$ For example, Al has demonstrated its aptitude for improving the efficiency of image analysis by quickly and accurately flagging specific anomalies for a radiologist's review. That type of automated analysis could find and match specific lung nodules (on chest CT images) between 62\% to $97 \%$ faster than a panel of radiologists. ${ }^{46}$ A subfield of artificial intelligence, machine learning, involves generating algorithms capable of knowledge acquisition through historical examples. ${ }^{45}$ IBM Watson Health (International Business Machines Corp., Armonk, N.Y.) cognitive computing system has used machine learning approaches to create a decision support system for physicians treating cancer patients, with the intention of improving diagnostic accuracy and reducing costs. ${ }^{47,48}$ Initially trained at Memorial Sloan Kettering Cancer Center using large volumes of patient cases and over 1 million scholarly articles, the project now has 14 participating cancer centers. All of these centers contribute to an ever-expanding corpus of information that helps Watson fine tune its ability to recommend optimal treatment options for cancer patients based on the nature of their specific illness. ${ }^{47,48}$ More recently, Royal Philips, together with its consortium partners, have received funding from the EU's Horizon 2020 program to pilot big data solutions to achieve better patient outcomes in healthcare at a lower cost. The three-year program is the largest EU-funded initiative to transform the region's healthcare sector through the use of big data. The program aims to improve patient outcomes and increase productivity in the health sector by applying Al technologies to complex datasets across the data value chain. This includes data from patients, healthcare providers, health insurers and medical technology providers. By bringing together all the key players from across health data value chains, the program seeks to break down the barriers between traditional healthcare silos and combine data sources to unlock new insights and enable collaborative innovation. It will also ensure the security and privacy of personal data is protected and managed within national and EU regulatory frameworks. With funding shared across 35 partners in 12 countries, the collaboration will combine insights from more than 11 million patients. It will be divided into three areas: Population Health and Chronic Disease Management, Oncology, and the Industrialization of Healthcare. With the majority of health budgets currently spent on treatment, the program will also implement pilots across the care continuum, from prevention to diagnosis, and treatment to home care.

In the field of plastic and reconstructive surgery, Al and deep learning can be applied in a variety of subspecialties including burn surgery and postoperative monitoring after microsurgery. An early application of machine learning related to plastic surgery was the development of a method to accurately determine healing time in burn injury. ${ }^{49}$ Using reflectance spectrometry and an artificial neural network, researchers developed a model to predict whether a burn would take more or less than 14 days to heal, 
ultimately serving as a proxy for the assessment of burn depth for surgical planning. ${ }^{50}$ Future use of Al in plastic surgery might be in providing the surgeons with quantitative support to aid decision-making and surgical actions.

\section{The Role of International and National Societies}

With the number of countries currently developing and conducting research with national registries, and significant steps being made towards the application of artificial intelligence in medicine and the surgical field, it is of utmost importance for the international plastic surgery community to unify and collaborate in order to improve care and add true value to our patients. The International Confederation of Plastic Surgery Societies (ICOPLAST) can play a significant role in leading this effort. ICOPLAST was formed in 2016 as a confederation of national plastic surgery societies; meaning that its members are national plastic surgery societies rather than individual plastic surgeons. ${ }^{51}$ To date, ICOPLAST has 62 members representing approximately 21,000 plastic surgeons. ${ }^{52}$

The mission of ICOPLAST is to:

1. Improve patient outcomes worldwide

2. Educate, communicate, advocate and advance the specialty of Plastic Surgery globally

3. Ensure that quality and safety are the essential components of reconstructive and aesthetic plastic surgery training and patient care

4. Encourage collaboration of national plastic surgery societies worldwide

In order to improve patient outcomes worldwide, quality data is needed. This can be achieved by internationally defined items and sharing of national country level registries in order to enhance patient care. Harmonization of databases utilized to study topics of interest to the plastic surgical society is of importance in order to correctly interpret the data. Serving as an umbrella organization, ICOPLAST can enhance the unification of defining a necessary item set (minimal dataset) for national databases to compare and contrast treatment patterns internationally in order to further improve breast reconstructive care. To achieve this, alignment of databases with clear and uniform definitions globally is of utmost importance. These data can be stored and analyzed into the ongoing current cloud-science efforts that are emerging globally. In the future, ICOPLAST can serve as a representation of the plastic surgery community worldwide. Future application might entail comparing and contrasting qualitatively good plastic surgical data on an international level, for example, with the use of Al or deep learning. 


\section{REFERENCES}

1. Bohl DD, Basques BA, Golinvaux NS, Baumgaertner MR, Grauer JN. Nationwide Inpatient Sample and National Surgical Quality Improvement Program give different results in hip fracture studies. Clinical orthopaedics and related research. 2014;472(6):1672-1680.

2. Bohl DD, Russo GS, Basques BA, et al. Variations in data collection methods between national databases affect study results: a comparison of the nationwide inpatient sample and national surgical quality improvement program databases for lumbar spine fusion procedures. The Journal of bone and joint surgery American volume. 2014;96(23):e193.

3. Quan H, Li B, Saunders LD, et al. Assessing validity of ICD-9-CM and ICD-10 administrative data in recording clinical conditions in a unique dually coded database. Health services research. 2008;43(4):1424-1441.

4. Global Big Data in Healthcare Market: Analysis and Forecast, 2017-2025. BIS Research. Available at: https://bisresearch.com/industry-report/global-big-data-in-healthcare-market-2025.html. Assessed July 6th 2018

5. "The Big-Data Revolution in US Health Care: Accelerating Value and Innovation", by Basel Kayyali, David Knott, and Steve Van Kuiken, McKinsey\& Company, April 2013, p. 5.

6. Exploring Data-Driven Innovation as a New Source of Growth: Mapping the Policy Issues Raised by "Big Data"', OECD, Digital Economy Papers, No. 222, 2013.

7. American Chamber of Commerce to the European Union. "Delivering the value of big data: EU policies for innovation and growth". Available at: http://www.amchameu.eu/sites/default/files/position_papers/ file_20131004_162938_MBhJxi_0.pdf. Assessed June 20th 2018.

8. European Commission - Press release. Artificial intelligence: Commission outlines a European approach to boost investment and set ethical guidelines. Available at: http://europa.eu/rapid/press-release_IP-183362_en.htm. Assessed June 9th 2018.

9. Mossialos E, Wenzl M, Osborn R, Sarnak D, editors. 2015 international profiles of health care systems. New York: The Commonwealth Fund; 2016.

10. Global observatory for eHealth [Internet]. Geneva: World Health Organization; 2015. Available from: http://www.who.int/goe/en/. Assessed June 2nd 2018.

11. Global diffusion of eHealth: making universal health coverage achievable. The third global survey on eHealth [Internet]. Geneva: World Health Organization; 2016. Available from: http://www.who.int/goe/ publications Assessed May 20th 2018.

12. Nilekani N. Building a foundation for better health: the role of the Aadhaar number. The National medical journal of India. 2011;24(3):133-135.

13. Bhan A, Bandewar SV. The UIDAI project: why some of the optimism might be nir-aadhar. The National medical journal of India. 2011;24(6):375-377.

14. Musen MA, van Bemmel J. Handbook of medical informatics. Houten: Springer. 1997.

15. HITECH Act. Available at: https://www.hhs.gov/hipaa/for-professionals/special-topics/hitech-actenforcement-interim-final-rule/index.html. Assessed July 9th 2018.

16. More than Half of Doctors Now Use Electronic Health Records Thanks to Administration Policies. The White House President Obama Blog by Jeanne Lambrew. Available at: https://obamawhitehouse.archives.gov/ blog/2013/05/24/more-half-doctors-now-use-electronic-health-records-thanks-administration-policies. Accessed July 9th 2018.

17. Office of the National Coordinator for Health Information Technology. 'Hospitals Participating in the CMS EHR Incentive Programs,' Health IT Quick-Stat \#45. dashboard.healthit.gov/quickstats/pages/FIGHospitals-EHR-Incentive-Programs.php. August 2017.

18. Office of the National Coordinator for Health Information Technology. 'Office-based Physician Electronic Health Record Adoption,' Health IT Quick-Stat \#50. dashboard.healthit.gov/quickstats/pages/physicianehr-adoption-trends.php. December 2016. 
19. eHealth Task Force Report "Redesigning health in Europe for 2020" (2012), available at http://www.ehealthcom.eu/fileadmin/user_upload/dateien/Downloads/redesigning_health-eu-for2020-ehtfreport2012_01.pdf. Assessed 4th of June 2018.

20. Directive 2011/24/EU of the European Parliament and of the Council of 9 March 2011 on the application of patients' rights in cross-border healthcare. Available at: https://eur-lex.europa.eu/legalcontent/EN/TXT/?uri=CELEX\%3A32011L0024. Assessed May 1st 2018.

21. European Health Parliament. Available at: http://www.healthparliament.eu/. Assessed July 1 st 2018.

22. Leitsalu L, Alavere H, Tammesoo ML, Leego E, Metspalu A. Linking a population biobank with national health registries-the estonian experience. Journal of personalized medicine. 2015;5(2):96-106.

23. The New York Times. "Millions of Anthem Customers Targeted in Cyberattack" Available at: https://www.nytimes.com/2015/02/05/business/hackers-breached-data-of-millions-insurer-says.html Assessed June 8th 2018.

24. BBC News. "Community Health Systems data hack hits 4.5 million" Available at: https://www.bbc.com/news/technology-28838661 Assessed July 9th 2018.

25. The Washington Post. "What you need to know about the massive hack that hit the British health-care system and elsewhere" Available at: https://www.washingtonpost.com/news/worldviews/wp/2017/ 05/12/what-you-need-to-know-about-the-massive-hack-that-hit-britain-and-11-othercountries/?utm_term=.a214f033fe88 Assessed July 102018.

26. Reuters. "Your medical record is worth more to hackers than your credit card" Available at: https://www.reuters.com/article/us-cybersecurity-hospitals/your-medical-record-is-worth-more-tohackers-than-your-credit-card-idUSKCNOHJ21I20140924 Assessed July 10th 2018. .

27. Health information \& the Law. "Who Owns Medical Records: 50 State Comparison". Available at: http://www.healthinfolaw.org/comparative-analysis/who-owns-medical-records-50-state-comparison Assessed July 10th 2018.

28. Mandl KD, Kohane IS. Time for a Patient-Driven Health Information Economy? The New England journal of medicine. 2016;374(3):205-208.

29. Mission statement. Hu-manity.co. Available at: https://hu-manity.org/. Assessed June 2nd 2018.

30. Davies J, Yudkin JS, Atun R. Liberating data: the crucial weapon in the fight against NCDs. The lancet Diabetes \& endocrinology. 2016;4(3):197-198.

31. Atun R. Time for a revolution in reporting of global health data. Lancet. 2014;384(9947):937-938.

32. The Lancet Diabetes E. Global health metrics and NCDs: are our perceptions years behind the data? The lancet Diabetes \& endocrinology. 2015;3(8):577.

33. Moorthy VS, Roth C, Olliaro P, Dye C, Kieny MP. Best practices for sharing information through data platforms: establishing the principles. Bulletin of the World Health Organization. 2016;94(4):234-234A.

34. The Belmont Report. Available at: https://www.hhs.gov/ohrp/regulations-and-policy/belmont-report/ Assessed: February 23rd 2017.

35. Brakewood B, Poldrack RA. The ethics of secondary data analysis: considering the application of Belmont principles to the sharing of neuroimaging data. Neurolmage. 2013;82:671-676.

36. Open Science Framework. The Transparency and Openness Promotion guidelines. Available at: https://cos.io/our-services/top-guidelines/ Assessed June 5th 2018.

37. Nosek BA, Alter G, Banks GC, et al. SCIENTIFIC STANDARDS. Promoting an open research culture. Science. 2015;348(6242):1422-1425.

38. Franco A, Malhotra N, Simonovits G. Social science. Publication bias in the social sciences: unlocking the file drawer. Science. 2014;345(6203):1502-1505.

39. Ong WL, Schouwenburg MG, van Bommel ACM, et al. A Standard Set of Value-Based Patient-Centered Outcomes for Breast Cancer: The International Consortium for Health Outcomes Measurement (ICHOM) Initiative. JAMA Oncol. 2017;3(5):677-685.

40. European Commission. "European Open Science Cloud (EOSC)". Available at: http://ec.europa.eu/ research/openscience/index.cfm?pg=open-science-cloud Assessed July 1st 2018. 
41. Australian Department of Education and Training. "2016 National Research Infrastructure Roadmap". Available at: https://www.education.gov.au/2016-national-research-infrastructure-roadmap Assessed July 10 th 2018

42. $\mathrm{NCl}$ Cancer Genomics Cloud Pilots. National Cancer Informatics. 2013. Available at: https://cbiit.nci.nih.gov/ncip/nci-cancer-genomics-cloud-pilots/nci-cloud-initiative. Accessed 10 June 2018.

43. CNBC news. "Facebook sent a doctor on a secret mission to ask hospitals to share patient data" Available at: https://www.cnbc.com/2018/04/05/facebook-building-8-explored-data-sharing-agreement-withhospitals.html. Assessed July 102018.

44. Bellman R. An Introduction to Artificial Intelligence: Can Computers Think? San Francisco: Boyd \& Fraser Pub Co; 1978.

45. Hashimoto DA, Rosman G, Rus D, Meireles OR. Artificial Intelligence in Surgery: Promises and Perils. Annals of surgery. 2018;268(1):70-76.

46. Koo CW, Anand V, Girvin F, et al. Improved efficiency of CT interpretation using an automated lung nodule matching program. AJR American journal of roentgenology. 2012;199(1):91-95.

47. Malin JL. Envisioning Watson as a rapid-learning system for oncology. Journal of oncology practice. 2013;9(3):155-157.

48. Oncologists partner with Watson on genomics. Cancer discovery. 2015;5(8):788.

49. Yeong EK, Hsiao TC, Chiang HK, Lin CW. Prediction of burn healing time using artificial neural networks and reflectance spectrometer. Burns : journal of the International Society for Burn Injuries. 2005;31(4):415-420.

50. Kanevsky J, Corban J, Gaster R, Kanevsky A, Lin S, Gilardino M. Big Data and Machine Learning in Plastic Surgery: A New Frontier in Surgical Innovation. Plastic and reconstructive surgery. 2016;137(5):890e897e.

51. The International Confederation of Plastic Surgery Societies (ICOPLAST). Website: http://www.icoplast.org/.

52. Rakhorst HA, Badran H, Clarke HM, et al. Introducing the International Confederation of Plastic Surgery Societies: ICOPLAST. Plastic and reconstructive surgery. 2017;140(3):627-633. 


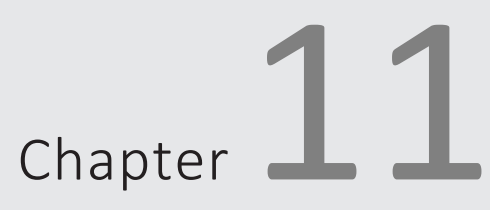

Summary 



\section{EVALUATING TRENDS}

Using big data collected from the largest administrative database in the United States, the National Inpatient Sample database (NIS), we evaluated the national and regional trends of different breast reconstruction techniques. In chapter 1 , we evaluated the in the use of immediate (one-stage) or delayed (two-stage) implant-based breast reconstruction over a period of 15 years. For women opting for mastectomy after breast cancer, the preferred method of postmastectomy breast reconstruction is implant-based. ${ }^{1}$ Traditionally, implant-based breast reconstruction is performed in a two-stage fashion. In the first stage, a tissue expander is placed followed by replacement of the tissue expander with a permanent implant (saline or silicone) in the second stage. ${ }^{2}$ Due to improved surgical technique, various studies report a significant increase in immediate one-stage implantation of a permanent implant. ${ }^{3-10}$ Although numerous studies have consistently reported successful clinical outcomes following either one-stage or two-stage implant-based breast reconstruction, a large-scale study focused on overall and regional trends in this type of reconstruction is lacking. In this chapter, we analyzed over 190,000 patients and evaluated national and regional trends in postmastectomy implant-based breast reconstruction. Although both one- and twostage procedures showed a significant rise, this study revealed a significantly higher increase in two-stage procedures, both nationally and regionally in the United States. Moreover, this study demonstrated that differences in sociodemographic and hospital variables affect the type of procedure performed in different regions of the United States. In chapter 2 we evaluated the national and regional trends in the United States over a period of 5 years using different modalities of autologous breast reconstruction. Besides implant-only breast reconstruction, autologous breast reconstructive procedures have evolved to incorporate several options. Globally, DIEP flap breast reconstruction is the gold standard for autologous breast reconstruction. This study demonstrated that this flap is not used as a primary flap for breast reconstruction in all regions. Moreover, differences in sociodemographic and hospital variables were seen between the different types of autologous breast reconstructions done.

\section{LAWS AND LEGISLATION}

Big data and large-volume databases can also be used to examine the effect of a law or legislation. In the United States, the Women's Health and Cancer Right Act (WHCRA) was federally enacted in 1998 to ensure that all group and individual health plans provide insurance coverage for reconstruction after mastectomy regardless of diagnosis. ${ }^{11}$ This legislation represented a paradigm shift in the availability of reconstruction by eliminating economic hurdles to care. Although a greater number of women have opted for breast reconstruction in the years after the WHCRA, this trend 
was not consistent across all demographic groups. Significant disparities exist based on access to health care, age, race, geographic location, and patient education. ${ }^{12-21}$ Moreover, a large body of available literature suggests that insurance status plays a key role in patient access to immediate breast reconstruction. ${ }^{12,13,16-18,22-24}$ These reports suggest that women with government owned Medicare healthcare insurance are significantly less likely to undergo breast reconstruction when compared to women with private insurance. That being said, these studies did not take age and related comorbidities into account. In chapter 3 we performed a matched cohort analysis to examine the differences between pre- and post-Medicare, and utilization of breast reconstruction. Using the NIS data for the years 1992 to 2013, we identified breast cancer patients undergoing immediate breast reconstruction. To minimize confounding medical variables, 64-year-old privately insured women were compared to 66-year-old Medicare-insured women. This chapter demonstrates a significant increase in immediate breast reconstruction rates in both cohorts. Moreover, after an initial slower upward trend, across a decade, immediate breast reconstruction in 66-year-old Medicare-insured women approached similar rates of breast reconstruction among those with private insurance. We demonstrated that in recent years, there have been no significant differences in the receipt of immediate breast reconstruction in both groups. One reason for the reported disparity in the literature may be due to the fact that women have been analyzed based on insurance type without regard for patient age and associated comorbidities.

\section{DISPARITIES}

Globally, the majority of patients diagnosed with breast cancer or DCIS are aged 60 years or older. ${ }^{25}$ With an estimated growth forecast of 57\% from 2010 to 2030, older patients with early stage breast cancer constitute the most rapidly growing breast cancer demographic. ${ }^{25,26}$ Over the last decades, survival rates following breast cancer diagnosis have improved significantly due to advances in screening and treatment. As patients are living longer after breast cancer treatment, a stronger emphasis has been placed on improving quality of life. Although treatment recommendations and postmastectomy breast reconstruction have been advocated for all women with early stage breast cancer, only $4.1-14 \%$ of older women (>60 years of age) undergo postmastectomy breast reconstruction. ${ }^{27-29}$ Significant differences exist in the "elderly" patient population although some may suffer with worsening health, many older women are still active and healthy. In Chapter 4, we used a clinical database, the ACSNSQIP database, to analyze the 30-day complication rates in an older patient population undergoing breast reconstruction. Using this clinical database, we identified 4450 breast cancer patients and 1104 DCIS patients for the years 2005 to 2014. We demonstrated that the rates of breast reconstruction decreased with increasing age. 
Yet, despite increasing age, associated complication rates remained stable. These finding suggest that immediate breast reconstruction in elderly breast cancer patients may be a viable option with an expected complication rate comparable to that in younger patients.

\section{BIG DATA RESEARCH AND TRADITIONAL OUTCOMES RESEARCH}

The advantages of using large-scale databases include timely and clinically relevant information. The huge samples provide statistical precision, external validity through less stringent selection of study subjects, and an economic means of investigating clinical questions. All forms of observational study design can be employed, including cross-sectional, cohort, case-controlled, case-crossover, and interrupted times series. That being said, each of these designs suffers from the same limitations as all nonexperimental research; namely the presence of unmeasured confounding factors that cannot be adjusted for. In chapter 5 we assessed the reliability of the American College of Surgeons National Surgical Quality Improvement Program (ACS-NSQIP) 30day perioperative outcomes and complications for immediate, autologous breast reconstruction by making a direct comparison to the 30-day and overall data of a high volume academic center. This chapter showed that the ACS-NSQIP may accurately represent the incidence of both 30-day and overall complications for surgical site infection, wound disruption and unplanned readmission, and as such be reliable for complication monitoring and comparison studies. However, this chapter found that the ACS-NSQIP may underreport readmissions and complications occurring beyond 30 days. Chapter 6 compared the rates of psychiatric disorder in patients undergoing free autologous breast reconstruction captured in the NIS and our institutional database. The prevalence of psychiatric disorders was similar between both datasets. However, both databases report a lower prevalence of psychiatric disorders (15.3\% and 17.6\%) than the average prevalence of mental health disorders in the general US female population, which is $21.8 \%$ as reported by the National Institution for Mental Health. This difference may be due to inherent limitations in reviewing the data in a retrospective fashion. Administrative databases are subject to variations in coding among providers, coding misclassification, and under-reporting. Possibly, the actual prevalence of psychiatric disorders- in both databases based on ICD-9 coding- might be under-reported and thus underestimated.

\section{COMPARING NATIONAL AND INTERNATIONAL DATASETS}

In breast cancer research, the most commonly used large national databases are the Surveillance, Epidemiology and End Result (SEER) program, the American College of 
Surgeons' National Surgical Quality Improvement Program (ACS-NSQIP) and the Nationwide Inpatient Sample (NIS) database. All three databases have previously been used to study trends in the surgical treatment of breast cancer and postmastectomy breast reconstruction. Moreover, these databases collectively offer unique opportunities to study patterns of care, variation in practice, and outcomes following surgical intervention. Research derived from these databases have played an increasing role in the development of clinical guidelines and health policy. Interestingly, these datasets had not yet been compared. Chapter 7 addresses this gap in knowledge by comparing these three databases. Patients with invasive breast cancer and DCIS were identified for the years 2005 - 2012. These databases collectively demonstrated a significant increase in immediate breast reconstruction; however, the extent of reporting of overall immediate breast reconstruction rates and of racial disparities differed significantly between databases. While the NIS and the ACS-NSQIP showed similar trends, the SEER showed significantly lower rates in several categories. This showed that the SEER database may not be generalizable to the entire U.S. population.

Globally there are a wide variety of clinical and administrative databases. The ability to compare these data across populations and time points can facilitate new scientific discoveries, faster access to treatment interventions, and enable differences between populations to be delineated. This in turn allows for optimization of treatment protocols and strategies, and subsequent improvement of quality of care. Yet, the ability to compare data between databases is greatly limited by the heterogeneity of data collected, their definitions, and coding. To date, no comparison between large databases has been made internationally within the field of breast reconstructive surgery. Chapter 8 is the first proof of concept study to explore the feasibility of combining two large existing operational databases in two countries; e.g. SEER database in the United States and NBCA database in the Netherlands. After comparing the databases on structure and content, the data variables were divided into general, treatment specific, cancer specific and follow-up variables and were matched. As proof of concept, mastectomy and immediate breast reconstruction rates in patients diagnosed with BC or DCIS were analyzed. 


\section{Nederlandse Samenvatting/ Dutch Summary}





\section{EVALUEREN VAN TRENDS}

Bij patiënten met borstkanker die borstamputatie ondergaan kunnen grofweg twee verschillende typen borstreconstructies uitgevoerd worden; reconstructie door middel van een borstprothese of lichaamseigen, autoloog, weefsel. Terwijl behandeling van borstkanker zeer geprotocolliseerd is, geldt dit niet voor borstreconstructie. Door gebruik te maken van de grootste administratieve database van de Verenigde Staten, de National Inpatient Sample Database (NIS), hebben wij de nationale en regionale trends van verschillende borstreconstructieve technieken in de Verenigde Staten geëvalueerd.

Over het algemeen wordt borstreconstructie door middel van een borstprothese het meest uitgevoerd. ${ }^{1}$ Tot op heden werd borstreconstructie met prothese uitgevoerd in twee stappen, ook wel bekend als de tweestapsmethode. In deze methode wordt er tijdens de borstamputatie operatie een tissue-expander geplaatst die na het oprekken van de huid in een aantal maanden in een tweede operatie (tweede stap) wordt vervangen door een definitieve prothese. ${ }^{2}$ In de afgelopen jaren zijn er, onder andere door verbeterde chirurgische technieken, uitstekende resultaten gerapporteerd met de eenstaps methode; het direct plaatsen van een definitieve borstprothese tijdens de borstamputatie operatie. ${ }^{3-10}$ Ondanks de toename van wetenschappelijke studies naar de superieure uitkomsten van de eenstaps methode, was er tot op heden geen grootschalig onderzoek naar de trends van deze twee verschillende technieken uitgevoerd. In hoofdstuk 1 hebben wij deze twee technieken vergeleken over een periode van 15 jaar waarbij we meer dan 190.000 borstkankerpatiënten hebben geanalyseerd. Dit hoofdstuk laat een significante stijging zien van zowel de eenstaps als tweestaps methode. Ondanks verbeterde chirurgische technieken en de internationale aandacht rondom de directe eenstaps, zagen wij een significant grotere stijging in het gebruik van de "traditionele" tweestaps methode. Daarnaast toonde deze studie aan dat verschillen in socio-economische, demografische- en ziekenhuisvariabelen van invloed zijn op het type reconstructie dat wordt uitgevoerd.

Als er na een borstamputatie besloten wordt om een autologe borstreconstructie te ondergaan, zijn er ook hier verschillende technieken waaruit gekozen kan worden. De Deep Inferior Epigastric Perforator (DIEP) lap borstreconstructie geldt hier als de gouden standaard. In hoofdstuk 2 hebben wij de nationale en regionale trends van de verschillende autologe borstreconstructies over een periode van 5 jaar geëvalueerd. Hoofdstuk twee toonde aan dat de DIEP-lap, ondanks de bewezen superioriteit aan andere autologe lappen, niet in alle regio's en staten wordt gebruikt als primaire autologe reconstructiemethode. Ook hier waren verschillende socio-economische, demografische en ziekenhuisvariabelen van invloed op het type autologe reconstructie dat werd uitgevoerd. 


\section{WET- EN REGELGEVING}

Het Amerikaanse zorgstelsel rust sterk op de particuliere zorgverzekering en is daarom vooral voor de rijkere Amerikanen betaalbaar en weggelegd. De private zorgverzekering vormt het belangrijkste deel van de dekking voor de meeste Amerikanen. Ongeveer 58 procent van de Amerikanen heeft een particuliere zorgverzekering en deze is in bijna alle gevallen via de werkgever of het pensioen betaald. Voor het overige deel van de bevolking (werklozen, zwakkeren en minder rijke ouderen) is de particuliere zorgverzekering vrijwel onbetaalbaar. Voor dit deel van de bevolking zijn er een tweetal door de staat gerunde zorgverzekeringen; Medicare voor de armere 65-plussers en Medicaid voor mensen of families met een laag inkomen, zwangere vrouwen of mensen met een handicap. De premies van Medicare en Medicaid worden gezamenlijk met, of geheel door, de staat betaald. De dekking van deze programma's zijn echter beduidend beperkter dan de private zorgverzekeringen.

Big data en grote administratieve en klinische databases kunnen worden gebruikt om het effect van nieuwe wet- en regelgeving te onderzoeken. Een goed voorbeeld van wet een regelgeving is de Women's Health and Cancer Right Act (WHCRA) wet van de Verenigde Staten. In 1998 is de WHCRA wet aangenomen in de Verenigde Staten. De WHCRA-wet dicteert dat ongeacht het type zorgverzekering, alle kosten van een borstreconstructie gedekt moet worden. ${ }^{11}$ Door deze wet werd borstreconstructie beschikbaar voor alle vrouwen met elk type zorgverzekering, ongeacht arm of rijk. In de jaren na de implementatie van de WHCRA-wet werd een significante stijging gezien in het aantal vrouwen die borstreconstructie ondergingen. Echter was deze trend niet gelijk in alle demografische groepen. Er werden zorgwekkende verschillen gerapporteerd in toegang tot gezondheidszorg, leeftijd, ras, geografische locatie van en voorlichting aan patiënten. ${ }^{12-21}$ Bovendien rapporteerden een groot aantal studies dat ondanks de WHCRA-wet, het type zorgverzekering wat men heeft van invloed is op het wel of niet ondergaan van borstreconstructie. ${ }^{12,13,16-18,22-24}$ Velen rapporteerden dat de armere 65-plussers met Medicare verzekering significant minder vaak borstreconstructies ondergingen in vergelijking met vrouwen met een privéverzekering. Bovenstaande studies hebben de Medicare-verzekerden vergeleken met alle vrouwen met privézorgverzekering, dus ook de jonge borstkanker populatie, en derhalve geen rekening gehouden met leeftijd gerelateerde comorbiditeiten en variabelen.

In hoofdstuk 3 hebben we beter gekeken naar deze groep door middel van een gematchte cohortanalyse. We hebben de NIS-database gebruikt en borstkanker patiënten met privézorgverzekering of Medicare zorgverzekering die tussen 1992 tot 2013 directe borstreconstructie hebben ondergaan met elkaar vergeleken. Om de leeftijd gerelateerde comorbiditeiten in acht te kunnen nemen, hebben we 64-jarigen met een privéverzekering vergeleken met 66-jarigen met een Medicare verzekering. Tussen 1992 en 2013 zagen we in beide groepen een significante toename in directe borstreconstuctie. Na een aanvankelijk tragere stijging in de Medicare-cohort zagen we 
dat na een aantal jaar het percentage van borstreconstructies in de Medicare-cohort nagenoeg was gelijkgetrokken met de privéverzekerden cohort. In de eerder gerapporteerde significante verschillen tussen Medicare-cohort en prive-cohort waren belangrijke variabelen zoals leeftijd en comorbiditeiten niet in acht genomen. We hebben in dit hoofdstuk laten zien dat ondanks eerdere rapportages en studies, de WHCRA wél een positief effect heeft gehad op toegang tot borstreconstructie na borstamputatie. Een belangrijk boodschap van deze studie is dat er goed moet worden gekeken naar alle variabelen wanneer er vergelijkingen gemaakt worden en uitspraken gedaan worden.

\section{ONGELIJKHEDEN EN VERSCHILLEN IN TOEGANG TOT ZORG}

Globaal gezien is borstkanker en ductaal carcinoma in situ (DCIS) de meest voorkomende vorm van kanker in vrouwen ouder dan 60 jaar. ${ }^{25}$ Door de wereldwijde vergrijzing is er voorspeld dat het aantal vrouwen met borstkanker zal gaan stijgen met 57\% tussen 2010 en 2030. ${ }^{25,26}$ De overlevingskansen van borstkanker patiënten zijn in de afgelopen decennia significant gestegen. Dit komt enerzijds doordat mensen door goede voorlichting eerder naar de huisarts gaan als zij iets aan hun borst merken. Maar het komt ook omdat er steeds betere onderzoeken en nieuwe behandelmethoden zijn. Gemiddeld is vijf jaar na diagnose $87 \%$ van de borstkanker patiënten nog in leven. Kwaliteit van leven na behandeling van borstkanker is een van de pilaren van borstkanker zorg. De behandeling van borstkanker gaat in principe volgens richtlijnen, ongeacht de leeftijd van de patiënt. Deze richtlijnen zijn geschreven op de gehele patiëntengroep. Tot deze behandelprotocollen behoort ook borstreconstructie na borstamputatie. In de westerse wereld ondergaat ongeveer $40-60 \%$ van de vrouwen borstreconstuctie. Bij vrouwen ouder dan 60 jaar ondergaat slechts 4,1 - 14\% borstreconstructie. ${ }^{27-29}$ Het grote verschil wordt onder andere toegeschreven aan de aanname dat oudere patiënten een hogere kans hebben op het krijgen van postoperatieve complicaties. Chirurgen en artsen bieden de optie tot het ondergaan van borstreconstructie daarom minder vaak aan. Juist op oudere leeftijd kunnen er grote gezondheidsverschillen zijn tussen mensen met dezelfde kalenderleeftijd.

In hoofdstuk 4 hebben we een klinische database, de ASC-NSQIP, gebruikt om de 30-daagse postoperatieve complicaties in patiënten die borstreconstructie ondergaan te analyseren. We hebben voor de jaren 2005 en 2014 totaal 4450 borstkankerpatiënten en 1104 DCIS-patiënten geïdentificeerd en de postoperatieve complicaties vergeleken. We zagen dat met toenemende leeftijd, het aantal vrouwen dat borstreconstructie ondergaat afneemt. Het krijgen van 30-daagse postoperatieve complicaties bleef echter stabiel, ondanks het toenemen van de leeftijd. Deze studie toont aan dat directe borstreconstructie bij de oudere borstkankerpopulatie als een serieuze optie overwogen dient te worden gezien het aantal complicaties gelijk is aan 
die van de jongere cohort. De behandeling en de optie om wel of geen borstreconstructie te ondergaan moet aan de persoonlijke situatie worden aangepast.

\section{BIG DATA ONDERZOEK EN TRADITIONELE ONDERZOEKSMETHODEN}

Big data biedt veel nieuwe kansen voor wetenschappelijk onderzoek. Grote hoeveelheden data, soms van de gehele populatie, kunnen bijdragen aan een betere generaliseerbaarheid van de uitkomsten van onderzoek. Anderzijds kun je vanuit grote hoeveelheden data goed focussen op specifieke subgroepen, wat kan leiden tot verbeteringen van personalized health, 'zorg op maat'. Wetenschappelijke kennis staat of valt bij de (mogelijkheden voor) verificatie en replicatie van de gevonden uitkomsten. Verificatie en replicatie zijn bij het gebruik van grote hoeveelheden data des te belangrijker vanwege de grotere kans op toevallige bevindingen (doordat meer data gemakkelijker beschikbaar zijn). Aan de ene kant geeft big dataonderzoek mogelijkheden voor verificatie- en replicatieonderzoek. $\mathrm{Er}$ is immers meer data beschikbaar en data kan makkelijker worden gekoppeld en vergeleken. Daarnaast kunnen alle vormen van observationeel studieontwerp worden gebruikt, inclusief crosssectionele studies, cohortstudies, case-controlled studies, case-crossover studies en tijdseries. Naast de vele voordelen kent big dataonderzoek zelf een aantal beperkingen, zoals het gegeven dat er correlaties mee aangetoond kunnen worden, maar niet of deze causaal of relevant zijn. Gegevens kunnen vrij gemakkelijk gemanipuleerd worden, en er kan bias en inconsistentie ontstaan. Big data moet daarom gezien worden als een aanvulling op het bestaande en zeker geen vervanging van onderzoek met bijvoorbeeld lokale databases en patientenpopulaties.

In hoofdstuk 5 hebben we de 30-dagen complicatie incidentie van directe autologe borstreconstucties van de ACS-NSQIP database vergeleken met een institutioneel database van een groot academisch centrum. Deze studie liet zien dat de ACS-NSQIP de incidentie van de 30-dagen postoperatieve complicaties, wonddehiscentie en ongeplande opnames goed weerspiegeld. De complicaties en heropnames die na 30dagen voorkomen bij autologe borstreconstructie worden echter niet gemonitord in de ACS-NSQIP.

In hoofdstuk 6 hebben we de percentages van de prevalentie van psychiatrische aandoeningen in patiënten die autologe borstreconstructie ondergaan vergeleken tussen de NIS en onze institutionele database. De prevalentie van psychiatrische stoornissen was vergelijkbaar tussen beide datasets. Beide datasets rapporteerden echter een lagere prevalentie van psychiatrische stoornissen dan de gemiddelde prevalentie in de algemene bevolking (15-17\% vs 21\%). Dit verschil kan te wijten zijn aan inherente beperkingen bij retrospectief onderzoek. Administratieve databases zijn onderhevig aan variaties in codering, misclassificatie en onderrapportage. Mogelijk is de 
feitelijke prevalentie van psychiatrische stoornissen - in beide databases op basis van ICD-9-codering - te laag gerapporteerd en dus onderschat.

\section{NATIONAAL EN INTERNATIONAAL VERGELIJKING VAN DATASETS}

In borstkankeronderzoek zijn de meest gebruikte grote nationale databases de Surveillance, Epidemiology and End Result (SEER) program, the American College of Surgeons' National Surgical Quality Improvement Program (ACS-NSQIP) en de Nationwide Inpatient Sample (NIS) database. Bovenstaande databases zijn gebruikt om trends en verschillen in de chirurgische behandeling van borstkanker en borstreconstructies na borstamputatie te bestuderen. Deze databases bieden een unieke mogelijkheid om patronen in de zorg, variatie in zorg en uitkomsten na chirurgische interventies te bestuderen. Wetenschappelijke studies die gebruik hebben gemaakt van de bovenstaande databases hebben een grote rol gespeeld bij de ontwikkeling van huidige klinische richtlijnen en beleidsvoering in de gezondheidszorg. Een voorbeeld hiervan is de genoemde Breast Cancer Patient Education Act in 2015. In de afgelopen jaren zijn er verschillende rapporten en wetenschappelijke studies gepubliceerd waaruit bleek dat ondanks de WHCRA-wet, vrouwen met een andere etnische achtergrond en vrouwen met lagere sociaaleconomische status verminderde toegang hebben tot zorg, en met name tot borstreconstructieve zorg, hebben. Om de verschillen in toegang tot zorg tegen te gaan is er in 2015 de Breast Cancer Patient Education Act aangenomen waarbij er nationaal miljoenen dollars werd uitgetrokken tot het creëren van awareness bij deze specifieke groepen. Er wordt hierbij gerefereerd naar meerdere studies die gebruik hebben gemaakt van de SEER-database. De andere veel gebruikte databases worden niet gebruikt om de argumentatie van de Breast Cancer Patient Education Act te ondersteunen.

De NIS, ACS-NSQIP en de SEER database geven allen aan dat zij een goede reflectie en representatie vormen van de gehele Amerikaanse populatie. Dit zou betekenen dat percentueel gezien het aantal en type operatieve behandelingen bij borstkanker patiënten overeen moeten komen. Echter waren deze databases tot op heden niet met elkaar vergeleken. In hoofdstuk 7 hebben we alle patiënten met de diagnose borstkanker of DCIS tussen 2005 en 2012 in de NIS, ACS-NQIP en SEER database geïdentificeerd en geanalyseerd. Tussen 2005 en 2012 zagen wij een significante toename in het aantal directe borstreconstructies in de drie databases; echter was het verschil in het percentage van borstreconstructies aanzienlijk groot tussen de databases. De NIS en den ACS-NSQIP toonden vergelijkbare trends. De SEER-database daarentegen liet lagere percentages zien in verschillende categorieën, onder andere het type reconstructie en ras. Deze studie laat zien dan de SEER-database, in tegenstelling tot wat wordt beweerd, niet generaliseerbaar is voor de gehele Amerikaanse bevolking 
en kan daarom niet als enige database gebruikt worden om statements te maken over de huidige staat van zorg.

Wereldwijd zijn er vele verschillende klinische en administratieve databases. De mogelijkheid om deze databases met elkaar te vergelijken, kan leiden tot verbeterde en snellere toegang tot behandelingsinterventies en nieuwe wetenschappelijke ontdekkingen. Dit kan op zijn beurt weer leiden tot optimalisatie van behandelprotocollen en strategieën en daaropvolgend voor verbetering van kwaliteit van zorg. Tot op heden is de mogelijkheid om data van verschillende databases te vergelijken beperkt door onder andere de heterogeniteit van dataelementen, definities en codering. Dusver is er geen internationale vergelijking gemaakt tussen twee grote nationale databases op het gebied van borstreconstructieve chirurgie. Hoofdstuk 8 is de eerste "proof of concept" studie waarbij is gekeken naar de mogelijkheid en haalbaarheid om twee nationale databases van twee verschillende landen met elkaar te vergelijken. We hebben hiervoor de SEER-database van de Verenigde Staten en de NBCA-database van Nederland met elkaar vergeleken. Als eerste stap hebben we de databases vergeleken op inhoud en structuur. Vervolgens hebben we de datavariabelen verdeeld in verschillende categorieën en deze categorieën met elkaar vergeleken; algemene variabelen, behandelingsvariabelen, kankervariabelen en follow-up variabelen. We hebben met deze eerste "concept" studie bewezen dan ondanks verschillen in database structuur en datavariabelen, vergelijking van twee nationale databases mogelijk is. Het samenvoegen van databases geeft een enorm potentieel voor verbering in patientenzorg. Het is dan ook van groot belang dat privacy wetgeving vergelijken van databases faciliteerd door het veld te vertellen hoe dit gedaan moet worden. Daarnaast is er een enorm verbeterpotentieel als we mensen die in twee databases van een land staan, bijvoorbeeld de borstkanker registratie en het borstimplantatenregister, op persoon kunnen herleiden om zo trends en verbindingen duidelijk te krijgen. 


\section{REFERENCES}

1. Albornoz CR, Bach PB, Mehrara BJ, et al. A paradigm shift in U.S. Breast reconstruction: increasing implant rates. Plastic and reconstructive surgery. 2013;131(1):15-23.

2. Serletti JM, Fosnot J, Nelson JA, Disa JJ, Bucky LP. Breast reconstruction after breast cancer. Plastic and reconstructive surgery. 2011;127(6):124e-135e.

3. Wagner JL, Fearmonti R, Hunt KK, et al. Prospective evaluation of the nipple-areola complex sparing mastectomy for risk reduction and for early-stage breast cancer. Annals of surgical oncology. 2012;19(4):1137-1144.

4. Gerber B, Krause A, Dieterich M, Kundt G, Reimer T. The oncological safety of skin sparing mastectomy with conservation of the nipple-areola complex and autologous reconstruction: an extended follow-up study. Annals of surgery. 2009;249(3):461-468.

5. Mallon P, Feron JG, Couturaud B, et al. The role of nipple-sparing mastectomy in breast cancer: a comprehensive review of the literature. Plastic and reconstructive surgery. 2013;131(5):969-984.

6. D'Souza N, Darmanin G, Fedorowicz Z. Immediate versus delayed reconstruction following surgery for breast cancer. The Cochrane database of systematic reviews. 2011(7):CD008674.

7. Ho G, Nguyen TJ, Shahabi A, Hwang BH, Chan LS, Wong AK. A systematic review and meta-analysis of complications associated with acellular dermal matrix-assisted breast reconstruction. Annals of plastic surgery. 2012;68(4):346-356.

8. Susarla SM, Ganske I, Helliwell L, Morris D, Eriksson E, Chun YS. Comparison of clinical outcomes and patient satisfaction in immediate single-stage versus two-stage implant-based breast reconstruction. Plastic and reconstructive surgery. 2015;135(1):1e-8e.

9. Han HH, Kim HB, Kim EK, Eom JS. Direct-to-Implant as a Frontline Option for Immediate Breast Reconstruction: A Comparative Study With 2-Stage Reconstruction. Annals of plastic surgery. 2018.

10. Ng WKY, Chesney A, Farrokhyar F, Hodgson N, Dal Cin A. One stage placement of permanent implant compared to two stage tissue expander reconstruction. Journal of plastic surgery and hand surgery. 2017;51(4):240-246.

11. Women's Health and Cancer Rights Act (WHCRA) Available at: https://www.congress.gov/bill/105thcongress/house-bill/616 Assessed June 1st 2018.

12. Hernandez-Boussard T, Zeidler K, Barzin A, Lee G, Curtin C. Breast reconstruction national trends and healthcare implications. The breast journal. 2013;19(5):463-469.

13. Yang RL, Newman AS, Lin IC, et al. Trends in immediate breast reconstruction across insurance groups after enactment of breast cancer legislation. Cancer. 2013;119(13):2462-2468.

14. Offodile AC, 2nd, Tsai TC, Wenger JB, Guo L. Racial disparities in the type of postmastectomy reconstruction chosen. The Journal of surgical research. 2015;195(1):368-376.

15. Wexelman B, Schwartz JA, Lee D, Estabrook A, Ma AM. Socioeconomic and geographic differences in immediate reconstruction after mastectomy in the United States. The breast journal. 2014;20(4):339346.

16. Mahmoudi E, Giladi AM, Wu L, Chung KC. Effect of federal and state policy changes on racial/ethnic variation in immediate postmastectomy breast reconstruction. Plastic and reconstructive surgery. 2015;135(5):1285-1294.

17. Albornoz CR, Bach PB, Pusic AL, et al. The influence of sociodemographic factors and hospital characteristics on the method of breast reconstruction, including microsurgery: a U.S. population-based study. Plastic and reconstructive surgery. 2012;129(5):1071-1079.

18. Polednak AP. Geographic variation in postmastectomy breast reconstruction rates. Plastic and reconstructive surgery. 2000;106(2):298-301

19. Kamali P, Koolen PG, Ibrahim AM, et al. Analyzing Regional Differences over a 15-Year Trend of OneStage versus Two-Stage Breast Reconstruction in 941,191 Postmastectomy Patients. Plastic and reconstructive surgery. 2016;138(1):1e-14e. 


\section{Chapter 11}

20. Vargas CR, Ganor O, Semnack M, Lin SJ, Tobias AM, Lee BT. Patient preferences in access to breast reconstruction. The Journal of surgical research. 2015;195(2):412-417.

21. Ashraf AA, Colakoglu S, Nguyen JT, et al. Patient involvement in the decision-making process improves satisfaction and quality of life in postmastectomy breast reconstruction. The Journal of surgical research. 2013;184(1):665-670.

22. Christian CK, Niland J, Edge SB, et al. A multi-institutional analysis of the socioeconomic determinants of breast reconstruction: a study of the National Comprehensive Cancer Network. Annals of surgery. 2006;243(2):241-249.

23. Roughton MC, DiEgidio P, Zhou L, Stitzenberg K, Meyer AM. Distance to a Plastic Surgeon and Type of Insurance Plan Are Independently Predictive of Postmastectomy Breast Reconstruction. Plastic and reconstructive surgery. 2016;138(2):203e-211e.

24. Hershman DL, Richards CA, Kalinsky K, et al. Influence of health insurance, hospital factors and physician volume on receipt of immediate post-mastectomy reconstruction in women with invasive and noninvasive breast cancer. Breast cancer research and treatment. 2012;136(2):535-545.

25. GLOBOCAN Breast Cancer Estimated Incidence, Mortality and Prevalence Worldwide in 2012. Available at: http://globocan.iarc.fr/old/FactSheets/cancers/breast-new.asp Assessed July 28th 2018.

26. Smith BD, Smith GL, Hurria A, Hortobagyi GN, Buchholz TA. Future of cancer incidence in the United States: burdens upon an aging, changing nation. Journal of clinical oncology : official journal of the American Society of Clinical Oncology. 2009;27(17):2758-2765.

27. Oh DD, Flitcroft K, Brennan ME, Spillane AJ. Patterns and outcomes of breast reconstruction in older women - A systematic review of the literature. European journal of surgical oncology : the journal of the European Society of Surgical Oncology and the British Association of Surgical Oncology. 2016;42(5):604615.

28. Bowman CC, Lennox PA, Clugston PA, Courtemanche DJ. Breast reconstruction in older women: should age be an exclusion criterion? Plastic and reconstructive surgery. 2006;118(1):16-22.

29. August DA, Wilkins E, Rea T. Breast reconstruction in older women. Surgery. 1994;115(6):663-668. 


\section{Valorization}



By law, Dutch universities have three main tasks: to educate at an academic level, to conduct scholarly research, and to ensure that research findings impact society. Valorization is the term that governmental and university policymakers use to denote this pros of "translating academic wisdom to societal benefit". This chapter outlines the valorization potential of the research findings presented in this thesis.

Data is the economy's new oil. It offers great potential to improve people's daily life, notably when it comes to their health. Harnessing the billions of terabytes of data generated across the world could radically transform healthcare, increase the quality of care and potentially save millions of lives. In this thesis, we used big data to elaborate on some of the non-clinical key questions related to breast reconstruction in patients diagnosed with breast cancer.

Do all breast cancer patients have the same access to high-quality care? Using big data, we evaluated the trend over time and examined disparities in different types of breast reconstructive techniques in chapter 1 and 2. Demonstrating significant disparities in different types of breast reconstruction performed throughout the United States, these studies showed that differences in sociodemographic and hospital variables affect the type of procedure performed in the different regions of the United States. Disparities come in different types and forms, including age-related disparities. We demonstrated this in chapter 4 where we evaluated if elderly breast cancer patients receive the same standard of care when compared to their younger counterparts. As physicians, we have the responsibility to provide the highest standard of care for each patient. In order to be aware of the shortcomings, we need data. In this thesis, we demonstrated that big data in the form of large-volume databases is an excellent tool to evaluate, assess, and demonstrate these shortcomings.

When we have a clear view of what must be improved to be able to provide the highest standard of care, the road opens for action. Healthcare policy is an excellent way to address and tackle these shortcomings. When laws or legislations are implemented, its effects can consequently be evaluated using big data, as we did in chapter 3. This chapter demonstrated that despite implementation of the WHCRA, significant disparities still exist. While an increasing number of women opted for breast reconstruction in the years after the WHCRA, this trend was not consistent across all demographic or racial groups. Having data, the community was able to assess what intervention was needed in order to reach the vulnerable target groups. As a result, the "Breast Cancer Patient Education Act of 2015" was introduced with the goal of increasing awareness on the availability and coverage of breast reconstruction, especially for racial and ethnic minority groups. This being said, we must keep in mind that the quality of data is important to draw the correct conclusions (Chapter 7). Moreover, in the final chapter of this thesis we conducted a proof of concept study comparing data between two different countries. The ability to compare these data across populations and time points can facilitate new scientific discoveries, faster access 
to treatment intervention, and enable differences between populations to be delineated.

The relevance of the outcomes of this dissertation of patients include better access to high-quality care. Policymakers can use the result of this dissertation implement laws and legislations to improve access to care and reduce disparities. The papers published in this thesis have led to different press-releases by the American Society of Plastic Surgery. As a result of this thesis, a symposium will be organized after the Ph.D. defense, bringing together different stakeholders in the field of big data and healthcare. As demonstrated in this dissertation, there is a great potential in big data. Big data should be embraced in order to improve patient care. 
Acknowledgement 

This thesis is the result of a journey that started in 2014. There have been many people that have walked alongside me during the last years and I'm grateful to all of them. I'd like to take this opportunity to express my gratitude to some of them in particular.

First and foremost, I would like to express my appreciation and gratitude to my supervisors.

Dear Professor Lin, dear Sam. When I first started at your research lab, I could barely understand the scientific language nor the art of doing research. You were patient, supportive, believed in me and gave me the tools to grow as a researcher and person. No idea was too crazy or out of reach. I actually don't think I have ever heard you saying "no" to any of the research idea's we had as a team. The words "sounds good, keep working hard", later abbreviated as a simple "SG, keep going" was the motto during my time in Boston, and I'm sure all the researchers in your lab will recognize these words. I never knew the true meaning of the word "mentor" until I started working for you. I now know that absolutely nothing is impossible, as long as you "work hard and keep going". Words can't express how grateful I am.

Dear Dr. Rakhorst. Dear Hinne. Even though there was a time difference between Boston and the Netherlands, you were always available to discuss ideas and projects and more importantly, helped expand the horizon. I always love to discuss and brainstorm with you. The sky is the limit! Thank you for all your support and guidance throughout the years. Your enthusiasm and "nothing is impossible" attitude has been an inspiration. I can't wait for our future projects together!

Professor van der Hulst, thank you for taking a chance on me and offering me this Ph.D.-position. You guided me throughout this whole process with enormous enthusiasm. Thank you for your support and guidance. I'm humbled to call you my mentor.

I would also like to thank all the members of my assessment committee, Prof. Dr. Vivianne Tjan-Heijnen, Prof. Dr. Andre Dekker, Prof. Dr. Lemmens, Dr. VranckenPeeters and Dr. Heuts, for taking the time reading and approving my dissertation. It is an honour to have experts as yourselves as my assessment committee.

Geoffrey Brahmer, a truly one in a kind person. I vividly remember that on my first day in Boston you sat me down in your office and told me that the things I will learn are not the things that I think I will learn. You were so right! You always found a way to teach me life-lessons. I hope you enjoy your well-deserved retirement and that you can fully focus on your books and photography!

I'd also like to take a moment to thank my paranymphs, Ahmed and Mehran.

Ahmed, when I arrived in Boston you were the first one making time to show me around the campus and giving me insight on life in Boston. In the last few years you have been the first one I reached out to for advice on research idea's. You shared your knowledge, your skills and kept saying that I was doing great, even though we both 
knew that at times it wasn't true. Thank you for revising all the papers before sending it to the big boss. Your kindness and work-ethic is truly inspiring and I'm happy to have made life-long friend.

Mehran, my dear brother. Thank you for the mental support during the last few years. I am truly blessed to have such an awesome brother. Every time I was swamped and absorbed in work, you always reminded me that there is more to life and to enjoy it a bit more.

A big thanks to the research team Alex, Bao, Babette, Marek, Charlotte, Max, Winona, Austin, Daniel, Callie, Abbas, Patrick, Sabine, Amnol, Pieter, Sabine, Michelle, Maaike, Sara, Annelotte, Michelle and Matt. You guys are really a dream team. I'm lucky to have worked with such amazing people. A special thanks to some of you specifically:

Alex, I really am blessed to call you my friend. You outdid yourself during your research year in Boston. I miss our hikes, our coffee dates and our endless talks on how we want our future to look like, and how we can leave our marks by making the world a better place. You are going to be an amazing plastic surgeon! Keep rocking!

Bao, no matter where we were, what the temperature was and what time a day it was, you always managed to look like an absolute queen. I can't wait to see you again.

Marek, I still don't know how you do it. Flying back and forth from Poland every other week, for 5 years now! I think you spend more time in the airplane than anyone else in the world! Thank you for being an amazing colleague and for teaching me the ins and outs of microsurgery.

Babette, for months we were the only ones at the office. While trying to survive the extreme cold in Boston. Thank you for being an amazing colleague!

Charlotte, I still have your "little book of feminism" gift. I am happy I had such an energetic and positive "bubbly" colleague.

To the life-long friends I have made in Boston, the list is long and you know who you are. A special thanks to Issy, Femke, Manda, Balthasar:

Dear Issy, the first time we met was at the UNGA in New York. I could never imagine that we would be living together a few months later and working on projects that would end up being published at the World Bank! There are so many memories that I don't even know where to start. Thank you for bringing fruit, orange juice and making soup every time I was sick (I know, Boston winter and me were not really compatible). I miss our times at the Charles river, enjoying the view and talking about life and work. It was then I said that there will be a time that we will reminisce our time in Boston as the best times in our life. Now that we've stepped into "normal" life it is finally time to say: I told you so...

Femke! You are one hell of an inspiring power women! You are my example and you inspire me with your work ethic and the way that you always find new ways to develop and evolve yourself. I miss our dinner dates and drinks, crazy adventures and trips to the nail salon. Can't wait for our future adventures! 
Sweet Manda; The first time we met was at the Kingsday party in Boston. With a huge smile you introduced yourself by saying. Oh well I'm not going to say it here but you know the story ;) That day was the beginning of a life-long friendship. As I've told you before, I admire your positive approach to life, the fact that you always no matter what, have a beautiful smile on your face. I am a blessed call you my friend!

Balthasar, I must admit that I miss your "oh that's because you're Persian" jokes. Thank you for the numerous coffee breaks at Café Nero.

A special thanks to my dear friend, Parisa. Even though there was an ocean between us, our friendship grew every second. Thank you for being my rock and for inspiring me by just being you.

Dena, Arghawan, Bamdad and Sohrab. well.. what is there to say. I'm a lucky girl to have you all in my life.

Dear Mom and Dad, Madar wa Pedare azizam

You left your country, your family and devoted your lives in order to offer us a better future. So that we would be able to grow up in a place where there is freedom and democracy. Thank you for all the sacrifices you have made. There are no words to express how much I love you and how much I look up to both of you. Throughout all the hardship in life, you kept the spirit high, studying and working hard, and giving back in any way you could. You both are an example that with hard work, you can be and do anything you want. Mitawan wa bayad!

Last but definitely not least, my dear Mehrdad. Thank you for standing by my side from the beginning. The last few years have been a roller-coaster in every sense of the word. Thank you for your love, support, faith and most of all, for your patience. You encouraged and supported me every step of the way. Can't wait to see what the future has to bring. The world is ours to conquer! 



\section{Curriculum Vitae}

Parisa Kamali Sadeghian was born on February $26^{\text {th }}$, 1988. Her parents, political refugees from Iran relocated to the Netherlands when she was 3 years old. After graduating from secondary school at Baken Park College, she attended medical school at the Vrije Universiteit Amsterdam, the Netherlands. Due to her upbringing and background, Parisa has always had a deep-rooted interest in human rights and humanitarian work. During medical school, she was an active member of the International Federation of Medical Student Association (IFMSA). She organized a variety of national and international conferences and attended multiple summer schools and extra-curricular internships related to (international) healthcare, global health and global surgery. At one of these extra-curricular internships in South-Africa, she was privy to the positive impact plastic surgery could have in helping victims of acid attacks, which in turn sparked her interest in the field.

Following graduation from medical school in 2014, she moved to Boston to start working as a research fellow in the Division of Plastic and Reconstructive Surgery at Harvard Medical School/Beth Israel Deaconess Medical Center under the supervision of Prof. Dr. Samuel Lin, MD, MBA, FACS. It was there that her passion for big data research was realized. She subsequently became involved in the work described in this thesis under the direct supervision of Dr. H.A. Rakhorst and Prof. dr. R.R.J.W. van der Hulst. During her time in Boston, she was honoured to work with inspirational leaders in the fields of big data and reconstructive surgery, published more than 30 international peer-reviewed articles and has presented her work in a variety of conferences globally. Moreover, her research has led to different awards, press releases and online videodiscussions. Parisa also started working as a volunteer for various global surgery NGO's, attending meetings at the United Nations, Capitol Hill, and a variety of global surgery related events. After spending 2 years in Boston, Parisa moved back to the Netherlands and started work as a resident (ANIOS) in Plastic and Reconstructive Surgery at the Radboud University Medical Center in Nijmegen, the Netherlands.

Parisa currently works as a resident (ANIOS) in Emergency Medicine at the Rode Kruis Hospital in Beverwijk. Moreover, she volunteers at the Medicine du Monde headquarters in Amsterdam. 



\section{List of publications}




\section{PUBLICATIONS USED IN THIS THESIS}

Kamali P., Koolen P.G., Ibrahim A., Paul, M., Dikmans R.E., Schermerhorn M., Lee B.T., Lin S.J.

Analyzing Regional Differences over a Fifteen-Year Trend of One-stage versus TwoStaged Breast Reconstruction in 941,191 Post-Mastectomy Patients.

Plast Reconstr Surg. 20162016 Jul;138(1):1e-14e.

Kamali P., Paul M.A., Ibrahim A.M., Koolen P.G., Wu W., Schermerhorn M., Lee B.T., Lin S.J.

National and Regional Differences in 32,248 Post-Mastectomy Donor Sites for Breast Reconstruction Patients Using the Updated National Inpatient Survey.

Ann Plast Surg. 2017 Jun;78(6):717-722.

Kamali P., Ricci J.A., Curiel DA, Cohen J.B., Chattha A., Rakhorst H.A., Lee B.T., Lin S.J. Immediate Breast Reconstruction among Patients with Medicare and Private Insurance: A Matched Cohort Analysis.

Plast Reconstr Surg Glob Open. 2018 Jan 12;6(1):e1552.

Kamali P., Curiel D., v. Veldhuisen C., Bucknor A.E., Lee B.T., Rakhorst H.A., Lin S.J.

Trends In Immediate Breast Reconstruction and Early Complication Rates Among Older Women: A Big Data Analysis.

J Surg Oncol. 2017 Jun;115(7):870-877.

Chen A.D., Kamali P., Chattha A.S., Bucknor A., Cohen J.B., Bletsis P., Flecha-Hirsch R., Tobias A., Lee B.T., Lin S.J.

The National Surgical Quality Improvement Program 30-day Challenge: Microsurgical Breast Reconstruction Outcomes Reporting Reliability.

Plast Reconstr Surg Global Open. 2018 Mar 6;6(3):e1643.

Becherer B.E., Kamali P., Paul M.A., Wu W., Curiel D.A., Rakhorst H.A., Lin S.J., Kansal K.J. Prevalence of Psychiatric Comorbidities among Women Undergoing Autologous Breast Reconstruction.

J Surg Oncol. 2017 Dec;116(7):803-810.

Kamali P., Zettervall S.L., Wu W., Ibrahim A., Medin C., Rakhorst H.A., Schermerhorn M., Lee B.T., Lin S.J.

Differences in the Reporting of Racial and Socioeconomic Disparities among Three Large National Databases for Breast Reconstruction.

Plast Reconstr Surg. 2017 Apr;139(4):795-807. 
Kamali P., van Bommel A.C.M., Becherer B., Cooter R., Mureau M.A.M., Pusic A., Siesling S., van der Hulst R.R.J.W., Lin S.J., Rakhorst H.A.

Immediate Breast reconstruction in the Netherlands and the United States: a Proof of Concept to Internationally Compare Quality of Care Using Cancer Registry Data In Press Plast Reconstr Surg

\section{OTHER PUBLICATIONS DURING PHD PERIOD}

Kamali P.*, Lee M.*, Becherer B.E., Wu W., Curiel D., Tobias A., Lin S.J., Lee B.T.

Medial Row Perforators Are Associated With Higher Rates Of Fat Necrosis in Bilateral DIEP Breast Reconstruction.

Plast Reconstr Surg. $2017 \mathrm{Jul} ; 140(1): 19-24$.

Kamali P., Lee M., Lee B.T.

Reply: Medial Row Perforators Are Associated With Higher Rates of Fat Necrosis in Bilateral DIEP Flap Breast Reconstruction.

Plast Reconstr Surg. 2017 Dec;140(6):819e-820e.

Kamali P.*, v. Veldhuisen C.*, Ibrahim A., Tobias A., Lee B.T., Lin S.J.

Prospective, Double-Blind Evaluation of Umbilicoplasty Techniques Using Conventional and Crowdsourcing Methods.

Plast Reconstr Surg. 2017 Dec;140(6):1151-1162.

Kamali P.*, v. Paridon M.W.*, Paul M.A., Winona W., Ibrahim A.M., Kansal K.J., Houlihan M.J., Morris D.J., Lee B.T., Lin S.J., Sharma R.

Oncoplastic Breast Surgery: Achieving Oncological and Aesthetic Outcomes.

J Surg Oncol. 2017 Aug;116(2):195-202.

Kamali P., Lin S.J.

Commentary on: Rates and Predictors of Readmission Following Body Contouring Procedures: An Analysis of 5100 Patients From The National Surgical Quality Improvement Program Database.

Aesthet Surg J. 2017 Mar 3. 2017 Sep 1;37(8):927-929.

Kamali P., van Paridon M.W., Ibrahim A.M., Paul M.A., Winters H.A., MartinotDuquennoy V.M., Noah E.M., Pallua N., Lin S.J.

Plastic Surgery Training Worldwide: Part 1. The U.S.A. and Europe.

Plast Reconstr Surg; Global Open. 2016 Mar;4(3)pe641.

Kamali P., Dean D., Skoracki R., Koolen P.G., Paul M.A., Ibrahim A.M.S., Lin S.J.

The Current Role of Three-Dimensional (3D) Printing in Plastic Surgery.

Plast Reconstr Surg. 2016 Mar;137(3):1045-55. 
Kamali P., Lin S.J.

Reply: The Current Role of Three-Dimensional Printing in Plastic Surgery".

Plast Reconstr Surg. 2017 Mar;139(3):812e-813e.

Kamali P., Lin S.J.

Book review: "Lymphedema: Presentation Diagnosis and Treatment."

Plast Reconstr Surg. 2016 May;137(5):1654-5.

Branford O.A., Kamali P., Rohrich R.J., James S.E., Song D.H., Mallucci P., Sun K., Stubican M., Lin S.J.

\#PlasticSurgery.

Plast Reconstr Surg. 2016 Dec;138(6):1354-1365.

Ricci J.A., Kamali P., Becherer B., Curiel D., Wu W., Tobias A.M., Lin S.J., Lee B.T.

Umbilical Necrosis Rates after Abdominal Based Microsurgical Breast Reconstruction.

J Surg Res. 2017 Jul;215:257-263.

Paul M.A., Kamali P., Shah J., Koolen P.G., Ibrahim A.M., Medin C., Lee B.T., Lin S.J.

Initial Assessment, Treatment and Follow-Up of Minor Pediatric Burn Wounds:

Utilization Of Personal Digital Devices In 21st Century.

Telemed J E Health. 018 May;24(5):379-385.

Knaus W.J., Kamali P., Chun Y., Lin S.J.

Breast Capsular Cerebrospinal Fluid Collection from Migration of a Ventriculoperitoneal Shunt Catheter.

Plast Reconstr Surg; Global Open. 2016 Mar;4(3)pe640.

Feldman H., Kamali P., Lin S.J., Halamka J.

Clinical 3D printing: A PHI and Compliance Perspective.

Int J Med Inform. 2018 Jul;115:18-23.

Paul M.A., Kamali P., Chen A.D., Ibrahim A.M., Wu W., Becherer B.E., Medin C., Lee B.T., Lin S.J.

Assessment of Functional Rhinoplasty with Spreader Grafting Using Acoustic Rhinomanometry and Validated Outcome Measurements.

Plast Reconstr Surg Global Open. 2018 Mar 19;6(3):e1615.

Bucknor A., Kamali P., Christensen J., Egeler S., van Velthuisen C., Rakhorst H., Matthijssen I., Lin S.J., Furnas H.

Gender Inequality for Women in Plastic Surgery: The Systematic Scoping Review.

Plast Reconstr Surg. 2018 Jun;141(6):1561-1577. 
Chattha A., Bucknor A., Kamali P., van Veldhuisen C.L., Flecha-Hirsch R., Bucknor A., Tobias A.M., Lee B.T., Lin S.J.

Comparison of Risk Factors and Complications in Patients by Stratified Mastectomy Weight: An Institutional Review of 1,041 Consecutive Cases

J Surg Oncol. 2017 Aug 22. 2017 Dec;116(7):811-818.

Bucknor A., Christensen J., Kamali P., Egeler S., van Veldhuisen C., Rakhorst H., Mathijssen I., Lin S.J., Furnas $\mathrm{H}$.

Crowdsourcing Public Perceptions of Plastic Surgeons: Is There a Gender Bias?

Plast Reconstr Surg Glob Open. 2018 Apr 16;6(4):e1728.

Taylor E.M., Wu W.W., Kamali P., Ferraro N., Upton J., Lin S.J., Iorio M.L.

Medial Femoral Condyle Flap Reconstruction of a Maxillary Defect with a 3-D Printing Template.

J Reconstr Microsurg Open. In press.

Tran B.N., Ruan Q.Z., Cohen J.B., Kamali P., Doval A., Tobias A.M., Singhal D., Lin S.J., Lee B.T.

Does hormone therapy use increase perioperative complications in abdominal based microsurgical breast reconstruction?

Plast Reconstr Surg. Plast Reconstr Surg. 2018 Jun;141(6):805e-813e.

Wu W.W., Medin C., Bucknor A., Kamali P., Lee B.T., Lin S.J.

Evaluating the Impact of Resident Participation and the July Effect on Outcomes in Autologous Breast Reconstruction.

Ann Plast Surg. 2018 Aug;81(2):156-162.

Bucknor A., Egelers E., Chen A., Chattha A., Kamali P., Brownstein G., Reed L., Watts D., Lin S.J.

National Mortality Rates After Outpatient Cosmetic Surgery and Low Rates of Perioperative Deep Vein Thrombosis Screening and Prophylaxis.

Plast Reconstr Surg. 2018 Mar 29. [Epub ahead of print]

Bletsis P., Bucknor A., Chattha A., Kamali P., Chen A., Flecha-Hirsch R., van der Lei B., Lee B.T., Lin S.J.

Evaluation of Contralateral and Bilateral Prophylactic Mastectomy and Reconstruction Outcomes: Comparing Alloplastic and Autologous Reconstruction.

Ann Plast Surg. 2018 Apr;80(4 Supp/ 4):S144-S149.

Bucknor A., Chattha A., Ultee K., Wu W., Bletsis P., Kamali P., Lee B.T., Lin S.J.

The Financial Impact and Drivers of Hospital Charges in Contralateral Prophylactic Mastectomy: A Nationwide Inpatient Sample Hospital Analysis.

Breast Cancer Res Treat. 2017 Jun 20. Sep;165(2):301-310. 
Tran B.N., Chen A.D., Kamali P., Singhal D., Lee B.T., Fukudome E.Y.

National perioperative outcomes of flap coverage for pressure ulcers from 2005 to 2015 using American College of Surgeons National Surgical Quality Improvement Program.

Arch Plast Surg. 2018 Sep;45(5):418-424.

Chi D., Curiel D., Bucknor A., Peymani A., Chattha A., Chen A.D., Bletsis P., Kamali P., Lin S. Institutional Collaboration in Plastic Surgery Research: A Solution to Resource Limitations.

Plast Reconstr Surg Glob Open. 2018 Jun 6;6(6):e1822.

Kamali P., Wu W., Lin S.J.

Chapter for the book: Oncoplastic and Reconstructive Management of the Breast: A Multidisciplinary Approach. Third Edition. Chapter 31: Nipple-areola complex reconstruction- with and without biologics. In press.

\section{ONLINE PUBLICATIONS}

Marks I., Kamali P., Khan M.A., et al.

Report "Data for the sustainable development of surgical systems: A global collaboration WDI surgical indicators data collection 2016. A collaborative project of the WHO, Stanford Medicine, the Lancet commission on global surgery, Kings College London, Incision and IFMSA."

Links: www.lancetglobalsurgery.org/indicators; www.data.worldbank.org

Kamali P., Marks I., Sama G., Vervoort D., Davies J.

Measuring surgical systems worldwide; an update

Worldbank blog.

Link: http://blogs.worldbank.org/opendata/measuring-surgical-systems-worldwide-update

Shah J., Kamali P., Lin S.J.

Virtual Reality Aided Reconstruction. PRS Tech Blog. February 2016.

Medin C., Ortiz E., Kamali P., Lin S.J.

Augmented Reality: Making Remote Surgical Assistance Possible. PRS Tech Blog. Augustus 2016.

\section{PRESS RELEASES}

The American Society of Plastic Surgeons (ASPS)

Differences in the Reporting of Racial and Socioeconomic Disparities for Breast Reconstruction. April 2017.

Link: http://bit.ly/2q80vfx 
\#PlasticSurgery. December 2016.

Link: http://bit.ly/2kMi1EV

Hot Topic Online Video Discussie door the Journal of Plastic and Reconstructive Surgery (PRS)

The Current Role of Three-Dimensional (3D) Printing in Plastic Surgery

By Rod J. Rohrich, MD, Editor in Chief J Plast Recon Surg

Link: http://bit.ly/2AEFb/D

Analyzing Regional Differences over a Fifteen-Year Trend of One-stage versus TwoStaged Breast Reconstruction in 941,191 Post-Mastectomy Patients

By Rod J. Rohrich, MD, Editor in Chief J Plast Recon Surg

Link: http://bit.ly/2AGgw2c

\#PlasticSurgery

By Fawn S. Hogan, MD, MSCl

Link: http://bit.ly/2kBSrDG

Differences in the Reporting of Racial and Socioeconomic Disparities for Breast Reconstruction

By Amy K. Alderman, MD, MPH, FACS

Link: http://bit.ly/2BgcPBq

Use of Crowd-Sourcing to Evaluate Three Different Techniques for Umbilical Reconstruction during DIEP Flap Procedure After Oncologic Mastectomy.

By Dr. Rod J. Rohrich, MD, Editor in Chief J Plastic Recon Surg

Link: http://bit.ly/2BVU9Uy

\section{ORAL PRESENTATIONS}

Kamali P., van Bommel A.C.M., Becherer B., Cooter R., , Mureau M.A.M., Pusic A., Siesling S., van der Hulst R.R.J.W, Lin S.J., Rakhorst H.A.

Immediate Breast Reconstruction in the Netherlands and the United States: A Proof of Concept to Compare Quality of Care Using Cancer Registry Data.

DICA congress, Amsterdam, The Netherlands, June 2018.

Kamali P., van Bommel A.C.M., Becherer B., Cooter R., , Mureau M.A.M., Pusic A., Siesling S., van der Hulst R.R.J.W, Lin S.J., Rakhorst H.A.

Immediate Breast Reconstruction in the Netherlands and the United States: A Proof of Concept to Compare Quality of Care Using Cancer Registry Data.

The European Association of Plastic Surgery Annual Meeting (EURAPS), Pisa, Italy, May 2017. 
Tran B.N., Kamali P., Fadayomi A., Lin S.J., Singhal D., Lee B.T.

Cost Analysis of Postmastectomy Reconstruction: A Comparison of Two Staged Implant Reconstruction using Tissue Expander and Acellular Dermal Matrix with Abdominal Based Perforator Free Flaps.

The European Association of Plastic Surgery Annual Meeting (EURAPS), Pisa, Italy, May 2017.

Tran B.N., Kamali P., Singh M, Singhal D., Rudd R, Lee B.T.

Readability, Complexity, and Suitability of Online Resources for Mastectomy and Lumpectomy.

The European Association of Plastic Surgery Annual Meeting (EURAPS), Pisa, Italy, May 2017.

Flescha-Hirsch R., Kamali P., Bucknor A., Chen A., Chattha A., Bletsis P., Lin S.J.

Anxiety, depression and secondary procedure rates after post-mastectomy breast reconstruction.

The European Association of Plastic Surgery Annual Meeting (EURAPS), Pisa, Italy, May 2017.

Kamali P., Lee M., Becherer B.E., Wu W., Curiel D., Tobias A., Lin S.J., Lee BT.

Medial Row Perforators Are Associated With Higher Rates Of Fat Necrosis in Bilateral DIEP Breast Reconstruction.

Academic Surgical Congress, Las Vegas, U.S., February 2017.

Ricci J.A., Kamali P., Becherer B., Curiel D., Wu W., Tobias A.M., Lin S.J., Lee B.T.

Umbilical Necrosis Rates after Abdominal Based Microsurgical Breast Reconstruction.

Academic Surgical Congress, Las Vegas, U.S., February 2017.

Kamali P., Lee M., Becherer B.E., Wu W., Curiel D., Tobias A., Lin S.J., Lee BT.

Medial Row Perforators Are Associated With Higher Rates Of Fat Necrosis in Bilateral DIEP Breast Reconstruction.

The Massachusetts Chapter of the American Society of Surgery. Boston, U.S., February 2016.

Kamali P., Lee M., Becherer B.E., Wu W., Curiel D., Tobias A., Lin S.J., Lee BT.

Medial Row Perforators Are Associated With Higher Rates Of Fat Necrosis in Bilateral DIEP Breast Reconstruction.

Northeastern Society of Plastic Surgery. Baltimore, U.S., October 2016.

Kamali P., van Bommel A.C.M., Becherer B.E., Lee B.T., Lin S.J., Rakhorst H.A.

Surgical Treatment and Reconstruction in Women Diagnosed With Breast Cancer: Making Registries Talk; Comparison Between Practices in USA and The Netherlands Using Big Data.

NVPC dagen, Rotterdam, NL, October 2016. 
Kamali P., Lee M., Becherer B.E., Wu W., Curiel D., Tobias A., Lin S.J., Lee BT.

Medial Row Perforators Are Associated With Higher Rates Of Fat Necrosis in Bilateral DIEP Breast Reconstruction.

NVPC dagen, Rotterdam, NL, October 2016.

Kamali P., Zettervall S.L., Wu W., Ibrahim A., Medin C., Rakhorst H., Schermerhorn M., Lee B.T., Lin S.J.

Differences in the Reporting of Racial and Socioeconomic Disparities among Three Large National Databases for Breast Reconstruction.

The Grand Rounds Plastic Surgery. Beth Israel Deaconess Medical Center/Harvard Medical School. Boston, U.S., September 2016.

Kamali P., Lee M., Becherer B.E., Wu W., Curiel D., Tobias A., Lin S.J., Lee BT.

Medial Row Perforators Are Associated With Higher Rates Of Fat Necrosis in Bilateral DIEP Breast Reconstruction.

The Surgical Outcomes Analysis \& Research group, Beth Israel Deaconess Medical Center/Harvard Medical School, Boston, U.S., September 2016.

Kamali P., van Bommel A.C.M., Becherer B.E., Lee B.T., Lin S.J., Rakhorst H.A.

Surgical Treatment and Reconstruction in Women Diagnosed With Breast Cancer: Making Registries Talk; Comparison Between Practices in USA and The Netherlands Using Big Data.

The Surgical Outcomes Analysis \& Research group, Beth Israel Deaconess Medical Center/Harvard Medical School, Boston, U.S., September 2016.

Kamali P., Paul M.A., Ibrahim A., Koolen P., Wu W., Schermerhorn M., Lee B.T., Lin S.J.

National and Regional Differences in 32,248 Post-Mastectomy Donor Sites for Breast Reconstruction Patients Using the Updated National Inpatient Survey.

The British Association of Plastic and Reconstructive and Aesthetic Surgeons Annual Summer Meeting. Bristol, U.K., July 2016.

Becherer B.E., Kamali P., Paul M.A., Wu W., Curiel D.A., Rakhorst H.A., Lin S.J., Kansal K.J. Prevalence of Psychiatric Comorbidities among Women Undergoing Autologous Breast Reconstruction.

The British Association of Plastic and Reconstructive and Aesthetic Surgeons Annual Summer Meeting. Bristol, U.K., July 2016.

Kamali P., van Paridon M.W., Ibrahim A.M., Paul M.A., Winters H.A., MartinotDuquennoy V.M., Noah E.M., Pallua N., Lin S.J. Plastic Surgery Training Worldwide: Part 1. The U.S.A. and Europe.

NVPC dagen, Eindhoven, NL, May 2016. 
Kamali P., Paul M.A., Ibrahim A.M., Koolen P.G., Wu W., Schermerhorn M., Lee B.T., Lin S.J.

National and Regional Differences in 32,248 Post-Mastectomy Donor Sites for Breast Reconstruction Patients Using the Updated National Inpatient Survey.

The Joint Annual Meeting of 95th American Association of Plastic Surgeons (AAPS) and 61st Plastic Surgery Research Council (PSRC), New York, U.S., May 2016.

Kamali P., van Paridon M.W., Ibrahim A.M., Paul M.A., Winters H.A., MartinotDuquennoy V.M., Noah E.M., Pallua N., Lin S.J.

Plastic Surgery Training Worldwide: Part 1. The U.S.A. and Europe.

The Surgical Outcomes Analysis \& Research group, Beth Israel Deaconess Medical Center/Harvard Medical School, Boston, USA, March 2016.

Kamali P., Koolen P.G.L., Ibrahim A.M.S., Paul M.A., Shermerhorn M.L., Lee B.T., Lin S.J. National and Regional Differences in 32,248 Post-Mastectomy Donor Sites for Breast Reconstruction Patients Using the Updated National Inpatient Survey.

The Surgical Outcomes Analysis \& Research group, Beth Israel Deaconess Medical Center/Harvard Medical School, Boston, U.S., March 2016.

Kamali P., Koolen P.G.L., Ibrahim A.M.S., Paul M.A., Shermerhorn M.L., Lee B.T., Lin S.J. Regional and National Trends over 20 years in One-stage versus Two-staged Implant Based Breast Reconstruction.

American Society of Plastic Surgery (ASPS), Boston, U.S., October 2015.

Kamali P., Koolen P.G.L., Ibrahim A.M.S., Paul M.A., Shermerhorn M.L., Lee B.T., Lin S.J. Regional and National Trends over 20 years in One-stage versus Two-staged Implant Based Breast Reconstruction.

The 58th annual meeting Ohio Valley Society of Plastic Surgeons, Covington, U.S., May 2015.

Kamali P., Koolen P.G.L., Ibrahim A.M.S., Paul M.A., Shermerhorn M.L., Lee B.T., Lin S.J. Regional and National Trends over 20 years in One-stage versus Two-staged Implant Based Breast Reconstruction.

The Surgical Outcomes Analysis \& Research group, Beth Israel Deaconess Medical Center/Harvard Medical School, Boston, U.S., June 2015. 


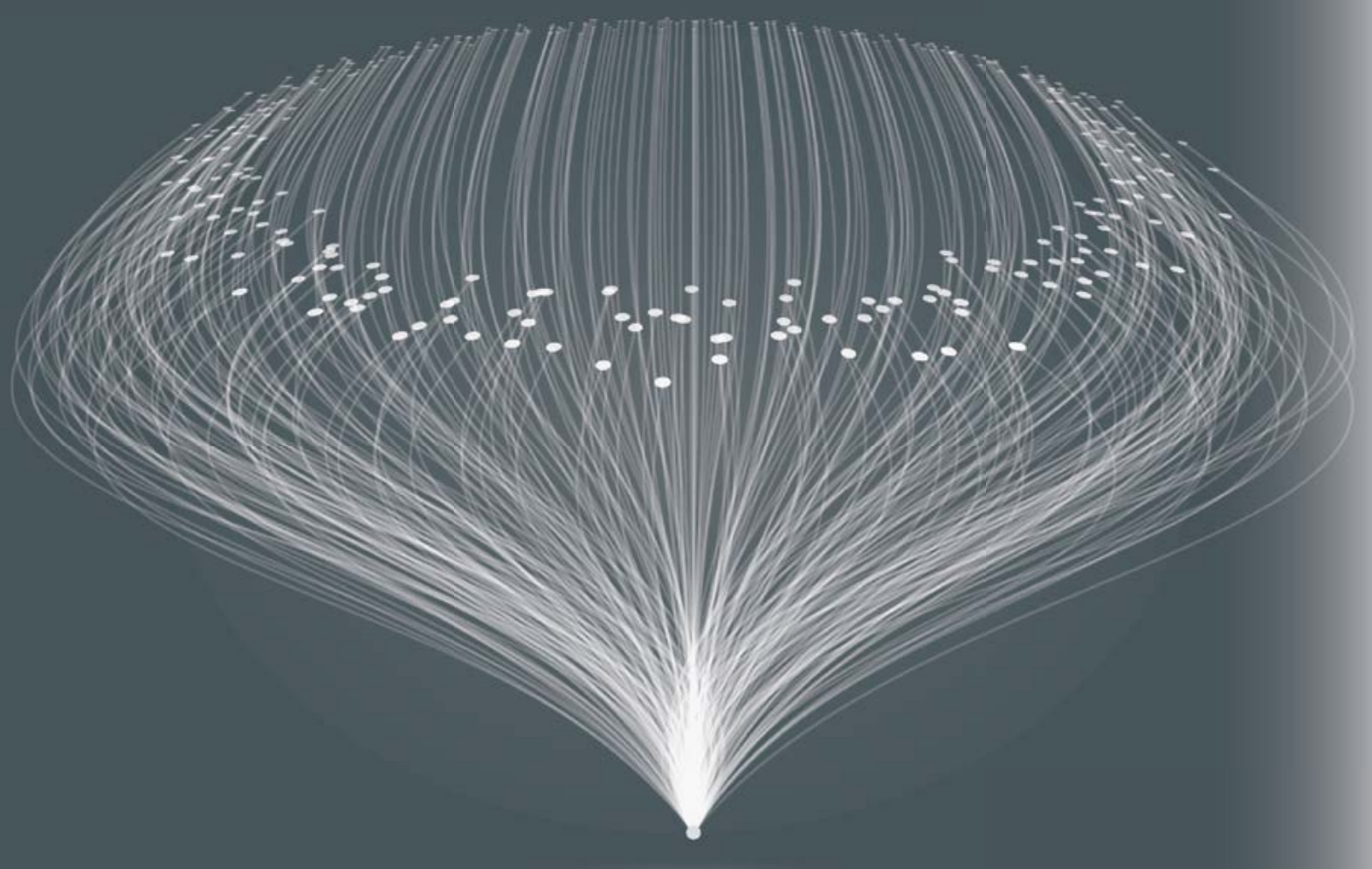

